\title{
Permuting actions, moment maps and the generalized Seiberg-Witten equations
}

\author{
Dissertation \\ zur Erlangung des mathematisch-naturwissenschaftlichen Doktorgrades \\ Doctor rerum naturalium \\ der Georg-August-Universität Göttingen \\ im Promotionsstudiengang Mathematical Sciences \\ der Georg-August University School of Science (GAUSS)
}

vorgelegt von

Martin Callies

aus Hamburg

Göttingen, 2015 
Betreuungsausschuss:

Prof. Dr. Victor Pidstrygach, Mathematisches Institut

Prof. Dr. Thomas Schick, Mathematisches Institut

$\underline{\text { Mitglieder der Prüfungskommission: }}$

Referent: Prof. Dr. Victor Pidstrygach, Mathematisches Institut

Korreferent: Prof. Dr. Thomas Schick, Mathematisches Institut

Weitere Mitglieder der Prüfungskommission:

Prof. Laura Covi, PhD, Institut für theoretische Physik

Prof. Dr. Thorsten Hohage, Institut für Numerische und Angewandte Mathematik

Prof. Dr. Ingo Witt, Mathematisches Institut

Prof. Chenchang Zhu, PhD, Mathematisches Institut

Tag der mündlichen Prüfung: 09.02.2016 


\begin{abstract}
In this thesis, we study properties and the geometry related to the generalization of the Seiberg-Witten equations introduced by Taubes and Pidstrygach. A crucial ingrediant to these equations is a hyperkähler manifold M with a permuting $S p(1)$ action. We study the differential forms induced on $\mathrm{M}$ and construct cocycles of degree 2 and 4 in the Cartan model for equivariant cohomology and the corresponding (generalizations of) moment maps in hyperkähler and multi-symplectic geometry. We generalize this and provide a natural and explicit construction of such a homotopy moment map for each cocycle in the Cartan model (of arbitrary degree). Coming back to the generalized Seiberg-Witten equations, we study properties of the generalized Dirac operator and provide new Lichnerowicz-Weitzenböck formulas in dimension 3. Finally, we give a list of examples of the generalized Seiberg-Witten equations, which have been studied in the literature.
\end{abstract}




\section{Acknowledgement}

At this point, I would like to thank all those people who supported me during the period of work on this thesis. First of all, I am grateful to my advisor Prof. Victor Pidstrygach for introducing me to many interesting questions and subjects and for his numerous helful comments and suggestions. I am also grateful to Prof. Thomas Schick for his commitment as co-supervisor. Thanks are also due to the DFG for financial support through the Research Training Group 1493 "Mathematical Structures in Modern Quantum Physics". Furthermore, I want to thank all participants of the "tea seminar", where many interesting topics and ideas were discussed. I am very grateful to Chris Rogers and Marco Zambon for very interesting discussions on $n$-plectic geometry and homotopy moment maps, which ultimately lead to the content of chapter 3. Moreover, I am grateful to Robin Raymond for reading a draft of this thesis. Special thanks are due to all my friends in and outside the mathematical institute, who made the time here in Göttingen very enjoyable, not only mathematically. Last but not least, I would like to thank my parents for their never ending support. 


\section{Contents}

1 Introduction 1

2 Hyperkähler manifolds and permuting actions 3

2.1 Hyperkähler manifolds . . . . . . . . . . . . . . . . . . . 3

2.1.1 Group actions and moment maps . . . . . . . . . . . . . . 6

2.1.2 Hyperkähler potential . . . . . . . . . . . . . . . . . . . 11

2.2 Hyperkähler manifolds with permuting actions . . . . . . . . . . . . . 11

2.2.1 Differential forms from permuting actions . . . . . . . . . . . 13

2.2.2 Modifying a permuting action . . . . . . . . . . . . 17

2.2.3 Permuting actions and the Cartan model . . . . . . . . . . . . . 19

2.2.4 Vector fields on hyperkähler manifolds with permuting action . . 25

2.2.5 Rotating $S^{1}$-actions from permuting actions . . . . . . . . . . 27

2.2.6 Forms and vector fields . . . . . . . . . . . . . . . . . . 29

2.2.7 Manifolds with hyperkähler potential . . . . . . . . . . . . . 31

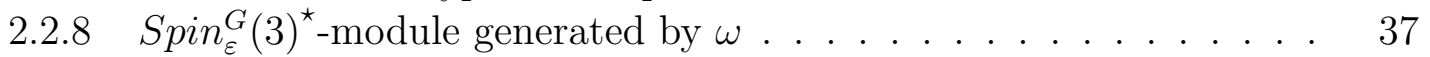

3 Homotopy moment maps and equivariant cohomology 41

3.1 Homotopy moment maps . . . . . . . . . . . . . . . . . . 41

3.2 Homotopy moment maps from degree 4 cocycles in the Cartan model . . 43

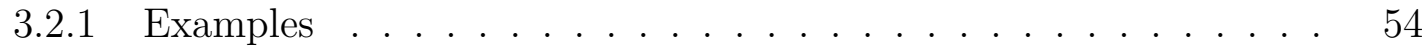

3.3 Homotopy moment maps and equivariant cohomology . . . . . . . . . 55

3.3.1 Differential forms on simplicial manifolds . . . . . . . . . . . . 55

3.3.2 Homotopy moment maps and the Bott-Shulman-Stasheff complex 57

3.3.3 Homotopy moment maps and Bott-Shulman-Stasheff cocycles . . 59

3.3.4 Simplicial differential forms . . . . . . . . . . . . . . . . . 61

3.3.5 Cartan complexes . . . . . . . . . . . . . . . . 62

3.3.6 The Cartan map . . . . . . . . . . . . . . . . 64

3.3.7 Cartan complex and Bott-Shulman-Stasheff complex . . . . . . . 65

3.3.8 Homotopy moment maps from Cartan cocycles . . . . . . . . . . . 66

4 The generalized Dirac operator $\quad 73$

$4.1 \operatorname{Spin}_{\varepsilon}^{G}(m)$-structures and spinors . . . . . . . . . . . . . 73

4.2 Connections and covariant derivatives . . . . . . . . . . . . . 75

4.2 .1 Gauge group . . . . . . . . . . . . . . . . . . . . . . . . . . . . . . . . . 75

4.3 Covariant derivative . . . . . . . . . . . . . . . . 75

4.4 Clifford multiplication and hyperkähler manifolds . . . . . . . . . . . 77 
4.5 Dirac operator . . . . . . . . . . . . . . . . 79

4.5.1 The linearized Dirac operator . . . . . . . . . . . . . 79

4.5.2 Dirac operators on manifolds with boundary . . . . . . . . . . 84

4.6 Examples . . . . . . . . . . . . . . . . . . . 86

5 The Seiberg-Witten equations $\quad 91$

5.1 Seiberg-Witten equations . . . . . . . . . . . . . . . . . 91

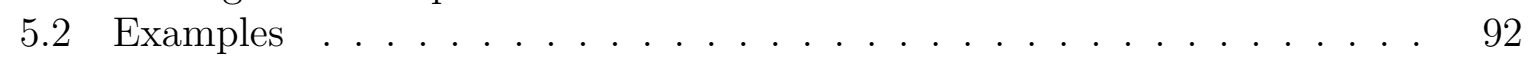

6 Lichnerowicz-Weitzenböck formulae $\quad 99$

6.1 The covariant derivative, it's adjoint and the Laplacian . . . . . . . . . . 99

6.2 Lichnerowicz-Weitzenböck formulae and curvature identities . . . . . . . 103

6.3 Lichnerowicz-Weitzenböck formula . . . . . . . . . . . . . . 106

6.4 Lichnerowicz-Weitzenböck formula for the linearized Dirac operator . . . 107

$6.5 L^{2}$-Lichnerowicz-Weitzenböck formula . . . . . . . . . . . . . . . 108

6.6 Seiberg-Witten functional . . . . . . . . . . . . . . . . . 109

6.7 Lichnerowicz-Weitzenböck formulae in dimension 4 . . . . . . . . . . 110

$\begin{array}{lll}7 & \text { Conclusion } & 113\end{array}$

$\begin{array}{ll}\text { Bibliography } & 115\end{array}$ 


\section{Chapter 1}

\section{Introduction}

Starting with the work of Donaldson ([Don83]), gauge theory proved to be a very useful tool in the study of the (smooth) geometry of 4-manifolds. The anti-selfduality equations also allowed him to define the Donaldson polynomials, which provide invariants of smooth 4-manifolds. In particular, he used these to prove the existence of exotic smooth structures in dimension 4 ([Don87]). The Seiberg-Witten equations ([SW94]) later made it possible to reprove many results obtained using Donaldson theory. Since an abelian structure group is used, these proofs are often easier than the original ones using Donaldson theory. A similar set of differential equations, the $P U(2)$-monopole equations, were used by Pidstrygach and Tyurin ([PT95]) to find a link between the Donaldson polynomials and the Seiberg-Witten invariants.

More recently, less well known examples like the Vafa-Witten equations ([VW94]) and the complex anti-selfduality equations gained interest, as they are closely related to gauge theory in higher dimensions. On the other hand, the Pin(2)-monopole equations were used by Manolescu ([Man16]) to disprove the triangulation conjecture.

All of these differential equations are examples of the generalized Seiberg-Witten equations, which were introduced by Taubes ([Tau99]) in dimension 3 and Pidstrygach ([Pid04]) in dimension 4 and also studied in [Hay06], [Sch10], [Cal10]. An important ingredient to these is a hyperkähler manifold $M$ with a permuting $S p(1)=S U(2)$-action. After studying these actions in the first chapters, we will describe these generalized SeibergWitten equations in chapter 5 and explain how specific choices of the hyperkähler manifold $M$ and the permuting action lead to various well-studied differential equations.

In the chapter 2, we study the properties of hyperkähler manifolds $M$ with an isometric action of a Lie group. Besides the tri-hamiltonian action of a Lie group $G$, we focus on the case of a permuting action of the group $S p(1)=S U(2)$ ([Swa91], [BGM93]). One approach to understanding these actions is to study the differential forms obtained from the symplectic forms by applying (graded) derivations of $\Omega^{*}(M)$ which are induced by the group action. More precisely, those forms obtained by inserting the fundamental vector field for the action and taking Lie derivatives with respect to these and exterior derivatives, i.e. the $G^{\star}$-submodule of $\Omega^{*}(M)$ generated by the symplectic forms. In the case of a tri-hamiltonian action, this submodule is essentially determined by the moment 
map. In the case of a permuting action, it contains all the differential forms (with values in $G$-representations) that appear in [BGM93].

For a symplectic manifold $(M, \omega)$ with hamiltonian $G$-action and moment map $\mu$, Atiyah and Bott observed that $\omega-\mu$ is a degree 2 cocycle in the Cartan model $C_{G}(M)$ for equivariant cohomology. Similarly, a tri-hamiltonian acion on a hyperkähler manifold gives a cocylce for each of the three symplectic forms. In the case of a permuting $S p(1)$ action, we show how some of the differential forms in the $S p(1)^{\star}$-module give rise to a similar cocylce in $C_{S p(1)}(M)$.

Another canonical differential form on a hyperkähler manifold $M$ is the 4 -form $\Omega=$ $\omega_{1} \wedge \omega_{1}+\omega_{2} \wedge \omega_{2}+\omega_{3} \wedge \omega_{3}$, which also admits extensions to degree 4-cocycles in the Cartan model for equivariant cohomology.

In chapter 3 , we interpret $\Omega$ as a multi-symplectic form (more precisely, a 3-plectic form) and show how the cocycles in the Cartan model for equivariant cohomology give rise to homotopy moment maps. These have been introduced and studied in [FRZ13] and provide a natural generalization of moment maps in symplectic geometry. We generalize this and show how cocycles of arbitrary degree give rise to homotopy moment maps.

In chapter 4, we return to the permuting actions on hyperkähler manifolds and explain how such an action can be used to construct a generalized Dirac operator (following [Tau99] and [Pid04]). In contrast to the previous literature, we allow the metric connection to have non-vanishing torsion. An example of a generalized Dirac operator of this sort is studied in [Sal13]. Chapter 5 is then concerned with the generalized Seiberg-Witten equations and various examples of these.

In chapter 6 , we focus on the 3-dimensional case and prove Lichnerowicz-Weitzenböck formulae for the generalized Dirac operator $\mathscr{D}_{A}$. In constrast to the case of usual Dirac operators, the generalized Dirac operator is a section in an infinite dimensional vector bundle and therefore not linear. This leads to three different Lichnerowicz-Weitzenböck formulae: one compares the non-linear Laplacian associated to the non-linear Dirac operator to the non-linear connection Laplacian, the second one is a LichnerowiczWeitzenböck formula for the linearization of the Dirac operator and the third one compare the squares of the norms of $\mathscr{D}_{A} u$ and $\nabla^{A} u$. 


\section{Chapter 2}

\section{Hyperkähler manifolds and permuting actions}

In this chapter we first recall the basic properties of a permuting action on a hyperkähler manifold. We then study the differential forms induced by such an action and show how these can be combined to give cocycles in degrees 2 and 4 in the Cartan model for equivariant cohomology. We also provide various equivalent conditions for the permuting action to induce a hyperkähler potential. We also describe the subspaces of differential forms generated by the symplectic forms and the action, both in the case when the action induces a hyperkähler potential as well as in the case when it does not.

\subsection{Hyperkähler manifolds}

2.1.1 Definition (Kähler manifold). An almost complex structure on a manifold $M$ is an endomorphism $I \in \Gamma(M$, End $(T M))$ satisfying $I^{2}=-\mathrm{id}_{T M}$. A Kähler manifold is a Riemannian manifold $\left(M, g^{M}\right)$ with a parallel (with respect to the Levi-Civita connection) orthogonal almost complex structure $I \in \Gamma(M$, End $(T M))$ such that the 2-form $\omega \in \Omega^{2}(M)$ is closed, where $\omega(v, w)=g^{M}(v, I(w))$ for all $v, w \in T_{x} M$. The symplectic form $\omega$ is called Kähler form.

2.1.2 Definition (hyperkähler manifold). A hyperkähler manifold is a Riemannian manifold $\left(M, g^{M}\right)$ with three parallel (with respect to the Levi-Civita connection) orthogonal almost complex structures $I_{1}, I_{2}, I_{3} \in \Gamma(M$, End $(T M))$ such that $I_{1} I_{2} I_{3}=-\mathrm{id}{ }_{T M}$ and $M$ is a Kähler manifold with respect to each of the three complex structures.

2.1.3 Remark ([Hit87, Lem. 6.8]). For $M$ to be hyperkähler, it is enough to require the existence of two anti-commuting orthogonal almost complex structures $I_{1}, I_{2} \in$ $\Gamma(M$, End $(T M))$ (define $\left.I_{3}:=I_{1} I_{2}\right)$ such that the three 2-forms are closed: $d \omega_{1}=d \omega_{2}=$ $d \omega_{3}=0$, where $\omega_{\ell}(v, w):=g^{M}\left(v, I_{\ell}(w)\right)$ for all $v, w \in T_{x} M$ and $\ell \in\{1,2,3\}$.

2.1.4 Remark (dimensions and holonomy groups). The existence of the three complex structures on a hyperkähler manifold $M$ implies that the dimension of $M$ is a multiple of 4 . 
Let $\mathbb{H}$ be the skew field of quaternions. As a vector space we identify $\mathbb{H} \cong \mathbb{R}^{4}$. The holonomy group of a $4 n$-dimensional hyperkähler manifold $M$ is contained in $S p(n) \subset$ $S O(4 n)$, where $S p(n)$ is the group of (right) $\mathbb{H}$-linear metric preserving automorphisms of $\mathbb{H}^{n}$. Conversely, every $4 n$-dimensional manifold with holonomy group contained in $S p(n) \subset S O(4 n)$ is a hyperkähler manifold.

The group $S p(1)$ can be identified with the sphere $S^{3}$ in the quaternions. We have an isomorphism $\mathbb{H} \supset S^{3} \rightarrow S p(1), q \mapsto L_{q}, L_{q}(h):=q h$ for $h \in \mathbb{H}$. From now on, we will use this isomorphism to identify $S p(1)$ with the sphere in the quaternions and its Lie algebra $\mathfrak{s p}(1)$ with the space of imaginary quaternions $\operatorname{Im}(\mathbb{H}):=\{h \in \mathbb{H} \mid \bar{h}=-h\}$. Throughout this text, we will also denote $\zeta_{1}:=i, \zeta_{2}:=j, \zeta_{3}:=k$.

2.1.5 Remark. Note that $S p(1)$ is isomorphic to $\mathrm{SU}(2)$ as well as to $\mathrm{Spin}(3)$, the simply connected double cover of $\mathrm{SO}(3)$.

2.1.6 Note (scalar multiplication). The tangent bundle of a hyperkähler manifold $M$ is a bundle of (left) $\mathbb{H}$-modules, i.e. we have a ring homomorphism called scalar multiplication

$$
\begin{aligned}
\mathcal{I}: \mathbb{H} & \rightarrow \Gamma(M, \text { End }(T M)), \\
h & \mapsto \mathcal{I}_{h},
\end{aligned}
$$

where $\mathcal{I}_{h}:=h_{0} \operatorname{id}_{T M}+h_{1} I_{1}+h_{2} I_{2}+h_{3} I_{3}$ for $h=h_{0}+h_{1} i+h_{2} j+h_{3} k$. In particular, for all $\zeta \in \operatorname{Im}(\mathbb{H})$ with $\|\zeta\|^{2}=1$ we have

$$
\mathcal{I}_{\zeta}^{2}=\mathcal{I}_{\zeta^{2}}=-\mathcal{I}_{\zeta \bar{\zeta}}=-\mathcal{I}_{1}=-\mathrm{id}_{M}
$$

This implies that $\mathcal{I}$ maps the sphere $S^{2} \subset \operatorname{Im}(\mathbb{H}) \subset \mathbb{H}$ into the space of complex structures on $M$. The scalar multiplication $\mathcal{I}$ is injective (if $\operatorname{dim}(M)>0$ ) and we have a sphere of complex structures $\left\{\sum_{\ell=1}^{3} \zeta_{\ell} I_{\ell} \mid \sum_{\ell=1}^{3} \zeta_{\ell}^{2}=1\right\}$. Unless mentioned explicitly, we shall therefore assume $\operatorname{dim}(M)>0$.

Note that we can also interpret $\mathcal{I}$ as a morphism from the trivial bundle with fibre $\mathbb{H}$ over $M$ into End $(T M)$.

We define a 2-form $\omega \in \mathfrak{s p}(1)^{\vee} \otimes \Omega^{2}(M)$ as follows:

$$
\langle\omega, \zeta\rangle:=\omega_{\zeta} \text { for all } \zeta \in \mathfrak{s p}(1)=\operatorname{Im}(\mathbb{H}),
$$

where $\omega_{\zeta}(v, w)=g^{M}\left(v, \mathcal{I}_{\zeta} w\right)$ for all $x \in M$ and $v, w \in T_{x} M$. If $\zeta \in \operatorname{Im}(\mathbb{H})=\mathfrak{s p}(1)$ is of norm one, $\|\zeta\|^{2}=1$, then $\mathcal{I}_{\zeta}$ is an (almost) complex structure and $\omega_{\zeta}$ the corresponding symplectic form.

2.1.7 Example. Consider $M=\mathbb{H}$ with the standard metric $g^{M}\left(v, v^{\prime}\right)=\operatorname{Re}\left(v \bar{v}^{\prime}\right)$ and complex structures given by

$$
I_{1}(v):=i v \quad I_{2}(v):=j v \quad I_{3}(v):=k v,
$$


for $v, v^{\prime} \in T_{h} \mathbb{H}=\mathbb{H}$. The scalar multiplication is given by $\mathcal{I}_{h^{\prime}}(v)=L_{h^{\prime}} v=h^{\prime} v$ for all $h^{\prime} \in \mathbb{H}, v \in T_{h} \mathbb{H}=\mathbb{H}$. The three symplectic forms $\omega_{\ell}=g^{M}\left(\cdot, I_{\ell}(\cdot)\right)$ for $\ell \in\{1,2,3\}$ are

$$
\begin{aligned}
& \omega_{1}=-d h_{0} \wedge d h_{1}-d h_{2} \wedge d h_{3}, \\
& \omega_{2}=d h_{1} \wedge d h_{3}-d h_{0} \wedge d h_{2}, \\
& \omega_{3}=-d h_{0} \wedge d h_{3}-d h_{1} \wedge d h_{2},
\end{aligned}
$$

where $h=h_{0}+i h_{1}+j h_{2}+k h_{3}$. Note that $i \omega_{1}+j \omega_{2}+k \omega_{3}=\frac{1}{2} d h \wedge d \bar{h}$.

In the same way, one also obtains the standard hyperkähler structure on $\mathbb{H}^{n}$.

2.1.8 Example. On the other hand, we can use multiplication from the right to define a hyperkähler structure on $M=\mathbb{H}$ using the complex structures:

$$
I_{1}(v)=-v i=v \bar{i} \quad I_{2}(v)=-v j=v \bar{j} \quad I_{3}(v)=-v k=v \bar{k}
$$

for $v \in T_{h} \mathbb{H}=\mathbb{H}$. The scalar multiplication is then given by $\mathcal{I}_{h^{\prime}}(v)=R_{\overline{h^{\prime}}} v=v \overline{h^{\prime}}$ for all $h, h^{\prime} \in \mathbb{H}, v \in T_{h} \mathbb{H}$. The corresponding three symplectic forms $\omega_{\ell}=g^{M}\left(\cdot, I_{\ell}(\cdot)\right)$ for $\ell \in\{1,2,3\}$ are

$$
\begin{aligned}
& \omega_{1}=d h_{0} \wedge d h_{1}-d h_{2} \wedge d h_{3} \\
& \omega_{2}=d h_{0} \wedge d h_{2}+d h_{1} \wedge d h_{3} \\
& \omega_{3}=d h_{0} \wedge d h_{3}-d h_{1} \wedge d h_{2}
\end{aligned}
$$

where $h=h_{0}+i h_{1}+j h_{2}+k h_{3}$. Note that $i \omega_{1}+j \omega_{2}+k \omega_{3}=\frac{1}{2} d \bar{h} \wedge d h$.

Also note that the induced orientation on $\mathbb{H} \cong \mathbb{R}^{4}$ is not the standard orientation of $\mathbb{R}^{4}$.

In the same way, one also obtains a hyperkähler structure on $\mathbb{H}^{n}$.

2.1.9 Remark. Note that the conjugation on $\mathbb{H}$ is a diffeomorphism

$$
\left(\mathbb{H}, L_{i}, L_{j}, L_{k}\right) \rightarrow\left(\mathbb{H}, R_{\bar{i}}, R_{\bar{j}}, R_{\bar{k}}\right)
$$

which intertwines the hyperkähler structures. While in most cases $\left(\mathbb{H}, L_{i}, L_{j}, L_{k}\right)$ is more convenient, it is in sometimes useful to consider $\left(\mathbb{H}, R_{\bar{i}}, R_{\bar{j}}, R_{\bar{k}}\right)$. For instance, when dealing with quaternionic matrices, acting on $\mathbb{H}^{n}$ as $\mathbb{H}$-linear maps. These act by standard matrix multiplication on $\left(\mathbb{H}, R_{\bar{i}}, R_{\bar{j}}, R_{\bar{k}}\right)$, but due to the non-commutativity of $\mathbb{H}$, the action on $\left(\mathbb{H}^{n}, L_{i}, L_{j}, L_{k}\right)$ is slightly more complicated.

2.1.10 Remark. Since we are interested in group actions on hyperkähler manifolds of a certain kind, which imply that the manifold is non-compact, we will mostly ignore compact examples like the $K 3$-surface and $4 n$-dimensional tori.

Further examples can be constructed using the hyperkähler reduction ([HKLR87, Thm. 3.2]), also cf. Example 2.1.22. Other constructions of hyperkähler metric use twistor methods ([HKLR87, Thm. 3.3] other examples include [Fei99], [Bie99]) 


\subsubsection{Group actions and moment maps}

2.1.11 Definition ([CE48]). Given a Lie algebra $\mathfrak{g}$, the Chevalley-Eilenberg differential is given by

$$
\begin{aligned}
& \delta_{\mathfrak{g}}: \bigwedge^{n}\left(\mathfrak{g}^{\vee}\right) \rightarrow \bigwedge^{n+1}\left(\mathfrak{g}^{\vee}\right) \\
& \left(\delta_{\mathfrak{g}} c\right)\left(\xi_{1}, \ldots, \xi_{k+1}\right):=\sum_{1 \leq i<j \leq n+1}(-1)^{i+j} c\left(\left[\xi_{i}, \xi_{j}\right], \xi_{1}, \ldots, \hat{\xi}_{i}, \ldots, \hat{\xi}_{j}, \ldots, \xi_{n+1}\right) .
\end{aligned}
$$

2.1.12 Remark. The complex $\left(\Lambda^{*}\left(\mathfrak{g}^{\vee}\right), \delta_{\mathfrak{g}}\right)$ computes the Lie algebra cohomology of $\mathfrak{g}$. Furthermore, note that if $\mathfrak{g}=\operatorname{Lie}(G)$ for some connected Lie group $G$, then $\left(\wedge^{*}\left(\mathfrak{g}^{\vee}\right), \delta_{\mathfrak{g}}\right)$ is isomorphic to the complex $\left(\Omega^{*}(G)^{G}, d\right)$ of left-invariant differential forms on the Lie group $G$.

2.1.13 Definition. Let $G$ be a Lie group acting on a manifold $M$. The infinitesimal action induces the following insertion operations:

$$
\begin{aligned}
\iota_{\mathfrak{g}}^{k}: \Omega^{*}(M) & \rightarrow \bigwedge^{k}\left(\mathfrak{g}^{\vee}\right) \otimes \Omega^{*-1}(M), \\
\iota_{\mathfrak{g}}^{k} \alpha\left(\xi_{1}, \ldots, \xi_{k}\right) & :=\iota_{v_{\xi_{k}}^{G}} \cdots \iota_{v_{\xi_{1}}^{G}} \alpha \in \Omega^{*-k}(M) \text { for } \alpha \in \Omega^{*}(M), \xi_{1}, \ldots, \xi_{k} \in \mathfrak{g} .
\end{aligned}
$$

Here $v^{G}$ is the fundamental vector field, i.e. $\left.v_{\xi}^{G}\right|_{x}:=\left.\frac{d}{d t} \exp (-t \xi) x\right|_{t=0}$, where $x \in M, \xi \in \mathfrak{g}$. Also, denote $\iota_{\mathfrak{g}}:=\iota_{\mathfrak{g}}^{1}$.

There is also a corresponding Lie derivative

$$
\begin{aligned}
\mathcal{L}_{\mathfrak{g}}: \Omega^{k}(M) & \rightarrow \mathfrak{g}^{\vee} \otimes \Omega^{k}(M), \\
\alpha & \mapsto \mathcal{L}_{\mathfrak{g}} \alpha,\left\langle\mathcal{L}_{\mathfrak{g}} \alpha, \xi\right\rangle:=\mathcal{L}_{v_{\xi}^{G}} \alpha \text { for } \xi \in \mathfrak{g} .
\end{aligned}
$$

We will also use the notation $\mathcal{L}_{\xi}:=\mathcal{L}_{v_{\xi}^{G}}$. As usual, $\iota_{\mathfrak{g}}$ and $\mathcal{L}_{\mathfrak{g}}$ are related by $\mathcal{L}_{\mathfrak{g}}=d \iota_{\mathfrak{g}}+\iota_{\mathfrak{g}} d$.

We use the same operations for differential forms with values in some $G$-module $V$.

2.1.14 Remark. Since $v^{G}: \mathfrak{g} \rightarrow \Gamma(M, T M)$ is $G$ equivariant, then $\iota_{\mathfrak{g}}^{k}$ and $\mathcal{L}_{\mathfrak{g}}$ map $G$ invariant differential forms with values in $V$ into $G$-invariant differential forms with values in $\mathfrak{g}^{\vee} \otimes V$, where $V$ is an arbitrary representation of $G$. Here, $\mathfrak{g}^{\vee}$ is understood as the coadjoint representation of $G$.

2.1.15 Remark. Note that if $G \curvearrowright M$ is a smooth action, $\rho: G \rightarrow$ Aut $(V)$ a representation and $\alpha \in \Omega^{p}(M, V)$ a differential form with values in $V$, then the infinitesimal version of $\alpha$ being $G$-invariant (i.e. $L_{g}^{*} \alpha=\rho(g) \alpha$ for all $g \in G$ ) is $\mathcal{L}_{\xi} \alpha=\rho_{*}(\xi) \alpha$ for all $\xi \in \mathfrak{g}$. Also note that if $G$ is connected, these two conditions are equivalent.

A special case of this, which will be crucial for the rest of this chapter, is the $k$-th power of the coadjoint representation $\mathfrak{g}^{\vee}=\operatorname{Lie}(G)^{\vee}$ of a Lie group $G$.

2.1.16 Lemma. Let $G \curvearrowright M$ be a smooth $G$-action and $\alpha \in\left(\left(\mathfrak{g}^{\vee}\right)^{\otimes k} \otimes \Omega^{p}(M)\right)^{G}$. Then

$$
\left\langle\mathcal{L}_{\mathfrak{g}} \alpha, \xi_{0} \otimes \cdots \otimes \xi_{k}\right\rangle=\left\langle\alpha, \sum_{\ell=1}^{k} \xi_{1} \otimes \cdots \otimes \xi_{\ell-1} \otimes\left[\xi_{0}, \xi_{\ell}\right] \otimes \xi_{\ell+1} \otimes \cdots \otimes \xi_{k}\right\rangle .
$$


In particular:

1. For $\alpha \in\left(\mathfrak{g}^{\vee} \otimes \Omega^{p}(M)\right)^{G}: \mathcal{L}_{\mathfrak{g}} \alpha=-\delta_{\mathfrak{g}} \alpha$.

2. For $\alpha \in\left(\mathfrak{g}^{\vee} \otimes \mathfrak{g}^{\vee} \otimes \Omega^{p}(M)\right)^{G}:\left\langle\mathcal{L}_{\mathfrak{g}} \alpha, \xi \otimes \xi^{\prime} \otimes \xi^{\prime \prime}\right\rangle=\left\langle\alpha,\left[\xi, \xi^{\prime}\right] \otimes \xi^{\prime \prime}\right\rangle+\left\langle\alpha, \xi^{\prime} \otimes\left[\xi, \xi^{\prime \prime}\right]\right\rangle$

Proof.

$$
\begin{aligned}
\left\langle\mathcal{L}_{\mathfrak{g}} \alpha, \xi_{0} \otimes \cdots \otimes \xi_{k}\right\rangle & =\mathcal{L}_{v_{\xi_{0}}^{G}}\left\langle\alpha, \xi_{1} \otimes \cdots \otimes \xi_{k}\right\rangle=\left.\frac{d}{d t}\left(L_{\exp \left(-t \xi_{0}\right)}\right)^{*}\left\langle\alpha, \xi_{1} \otimes \cdots \otimes \xi_{k}\right\rangle\right|_{t=0} \\
& =-\left.\frac{d}{d t}\left\langle\alpha, A d_{\exp \left(-t \xi_{0}\right)}\left(\xi_{1}\right) \otimes \cdots \otimes A d_{\exp \left(-t \xi_{0}\right)}\left(\xi_{k}\right)\right\rangle\right|_{t=0} \\
& =\left\langle\alpha, \sum_{\ell=1}^{k} \xi_{1} \otimes \cdots \otimes \xi_{\ell-1} \otimes\left[\xi_{0}, \xi_{\ell}\right] \otimes \xi_{\ell+1} \otimes \cdots \otimes \xi_{k}\right\rangle
\end{aligned}
$$

for all $\xi_{0}, \ldots \xi_{k} \in \mathfrak{g}$.

2.1.17 Remark. In particular, if $\mathfrak{g}=[\mathfrak{g}, \mathfrak{g}]$ (e.g. if $\mathfrak{g}$ is semisimple), then $\alpha \in\left(\mathfrak{g}^{\vee} \otimes\right.$ $\left.\Omega^{k}(M)\right)^{G}$ can be recovered from $\mathcal{L}_{\mathfrak{g}} \alpha$. Moreover, using the Cartan formula $\mathcal{L}_{\mathfrak{g}}=d \iota_{\mathfrak{g}}+\iota_{\mathfrak{g}} d$, we see that any closed form $\alpha \in\left(\mathfrak{g}^{\vee} \otimes \Omega^{k}(M)\right)^{G}$ is exact.

2.1.18 Definition (moment map). A smooth action of a Lie group $G$ on a symplectic manifold $(M, \omega)$ is said to be a symplectic action if it fixes the symplectic form $\omega$ (i.e. $L_{h}^{*} \omega=\omega$ for all $\left.h \in G\right)$. A smooth map $\mu: M \rightarrow \mathfrak{g}^{\vee}$ is said to be a moment map for the symplectic $G$-action on $M$ if

1. $d \mu=-\iota_{\mathfrak{g}} \omega$ (moment map condition),

2. $\mu(g x)=A d_{g}^{*}(\mu(x))$ for all $g \in G, x \in M$ (equivariance).

A hamiltonian action is a symplectic $G$-action which admits a moment map.

2.1.19 Definition (hyperkähler action). A smooth action of a Lie group $G$ on a hyperkähler manifold $\left(M, g^{M}, I_{1}, I_{2}, I_{3}\right)$ is said to be a hyperkähler action, if

1. $G$ acts isometrically, i.e. for all $h \in G: L_{h}^{*} g^{M}=g^{M}$,

2. $G$ fixes the symplectic forms, i.e. for all $h \in G: L_{h}^{*} \omega=\omega$.

In particular, in this situation, the induced $G$-action on $T M$ commutes with the complex structures.

The definition of a moment map for a hyperkähler action is analoguous to the definition for symplectic actions, but now we have to take care of three symplectic structures.

2.1.20 Definition. Let $\left(M, g^{M}, I_{1}, I_{2}, I_{3}\right)$ be a hyperkähler manifold with a hyperkähler action of a Lie group $G$. Consider the form $\omega \in \mathfrak{s p}(1)^{\vee} \otimes \Omega^{2}(M)$. A smooth map $\mu: M \rightarrow \mathfrak{g}^{\vee} \otimes \mathfrak{s p}(1)^{\vee}$ is said to be a hyperkähler moment map for the $G$-action on $M$ if

1. $d \mu=-\iota_{\mathfrak{g}} \omega$ (moment map condition), 
2. $\mu(g x)=A d_{g}^{\vee}(\mu(x))$ for all $g \in G, x \in M$ (equivariance).

A tri-hamiltonian action is a hyperkähler $G$-action which admits a moment map.

2.1.21 Remark. If $\mu: M \rightarrow \mathfrak{g}^{\vee} \otimes \mathfrak{s p}(1)^{\vee}$ is a hyperkähler moment map, then $d\langle\mu, \zeta\rangle=$ $-\iota_{\mathfrak{g}} \omega_{\zeta}$, and therefore $\langle\mu, \zeta\rangle: M \rightarrow \mathfrak{g}$ is a moment map for $\omega_{\zeta}$. In particular, let

$$
\mu_{1}:=\langle\mu, i\rangle, \quad \mu_{2}:=\langle\mu, j\rangle, \quad \mu_{3}:=\langle\mu, k\rangle .
$$

Then $\mu: M \rightarrow \mathfrak{g}^{\vee} \otimes \mathfrak{s p}(1)^{\vee}$ is a hyperkähler moment map if and only if $\mu_{1}, \mu_{2}, \mu_{3}$ are moment maps for $\omega_{1}, \omega_{2}, \omega_{3}$, respectively.

2.1.22 Example (hyperkähler reductions). Many known examples of hyperkähler manifolds can be obtained from quaternionic vector spaces (or subspaces of such) by hyperkähler reduction ([HKLR87]): Given a tri-hamiltonian action $G \curvearrowright M$ and $\xi \in$ $\left(\mathfrak{g}^{\vee}\right)^{G} \otimes \mathfrak{s p}(1)^{\vee}$ a regular value of the moment map and if $G$ acts freely on $\mu^{-1}(\xi)$, then $\mu^{-1}(\xi) / G$ is again a hyperkähler manifold. In many cases, it is also useful to allow $M$ to be infinite-dimensional (often $M$ is an infinite-dimensional quaternionic vector space).

Examples of this sort include

1. $G \subset S p(n) \curvearrowright \mathbb{H}^{n}=M$. For example, from various groups $G$, the following hyperkähler manifolds are obtained as hyperkähler quotients:

a) Calabi metric on $T^{*} \mathbb{C} P^{n}$ ([Cal79], description as hyperkähler quotient in [Fei99, Example 1.7]), generalizing the Eguchi-Hanson metric on $T^{*} \mathbb{C} P^{1}([\mathrm{EH} 78])$

b) Nakajima quiver varieties ([Nak94])

i. moduli space of framed instantons (of charge $k$ ) on $S^{4}$ ([AHDM78]),

ii. ALE spaces and moduli spaces of instantons on ALE spaces ([Nak94])

c) toric hyperkähler manifolds ([BD00])

2. spaces of solutions of Nahm's equations ([Nah82]), with various boundary conditions

a) cotangent bundles to complex semisimple Lie groups ([Kro88])

b) moduli space of Bogomolny monopoles ([Hit83], [Don84], [AH88][Prop 16.1])

c) ALE spaces (cf. [Kro89]), or more generally, intersections of complex coadjoint orbits with certain slices (cf. [Bie97])

d) coadjoint orbits of semisimple Lie groups (cf. [Kro90a], [Kro90b], [Biq96], [Kov96], overview in [Bie07][2.2]) 
2.1.23 Example (linear actions $\left.\boldsymbol{G} \rightarrow \boldsymbol{S p}(\boldsymbol{n}) \curvearrowright \mathbb{H}^{n}\right)$. Consider $\left(\mathbb{H}^{n}, R_{-i}, R_{-j}, R_{-k}\right)$ as a hyperkähler manifold as in Example 2.1.8. The moment map for the action $S p(n) \subset \operatorname{End}_{\mathbb{H}}\left(\mathbb{H}^{n}\right) \cong M_{n}(\mathbb{H}) \curvearrowright \mathbb{H}^{n}$ by matrix multiplication $(A, x) \mapsto A x$ is

$$
\begin{aligned}
& \mu: \mathbb{H}^{n} \rightarrow \mathfrak{s p}(1)^{\vee} \otimes \mathfrak{s p}(n)^{\vee}, \\
& \mu=\frac{1}{2} \iota_{\mathfrak{s p}(1)} \iota_{\mathfrak{g}} g, \text { i.e. }\langle\mu(x), \zeta \otimes \xi\rangle=\frac{1}{2} \operatorname{Re}\left(\zeta x^{*} \xi x\right) .
\end{aligned}
$$

A proof for this is given in [Sch10, Lem. 3.4.1] and also follows from Corollary 2.2.46 below.

We can also study any action $G \rightarrow S p(n) \curvearrowright \mathbb{H}^{n}$. The moment maps is given by composing $\mu$ with the dual $\mathfrak{s p}(n)^{\vee} \rightarrow \mathfrak{g}^{\vee}$ of the induced Lie algebra morphism $\mathfrak{g} \rightarrow \mathfrak{s p}(n)$. We will be mostly interested in the case of a subgroup $G \subset S p(n)$.

2.1.24 Remark. Whenever $\left(\mathbb{H}^{n}, L_{i}, L_{j}, L_{k}\right)$ is more convenient than $\left(\mathbb{H}^{n}, R_{-i}, R_{-j}, R_{-k}\right)$, we can use the isomorphism $\left(\mathbb{H}^{n}, R_{-i}, R_{-j}, R_{-k}\right) \rightarrow\left(\mathbb{H}^{n}, L_{i}, L_{j}, L_{k}\right), x \mapsto \bar{x}$ from Remark 2.1.9. The $S p(n)$-action on $\left(\mathbb{H}^{n}, L_{i}, L_{j}, L_{k}\right)$ then becomes

$$
(A, x) \mapsto \overline{A \bar{x}}=\left(x^{t} A^{*}\right)^{t},
$$

where $A^{*}$ denotes the (quaternionic) conjugated and transposed matrix.

2.1.25 Example $(U(1) \subset U(n) \subset S p(n))$. Consider the isomorphism of quaternionic vector spaces

$$
\begin{aligned}
\mathbb{C}^{n} \otimes_{\mathbb{C}} \mathbb{H} & \rightarrow \mathbb{H}^{n}, \\
v \otimes h & \mapsto v h .
\end{aligned}
$$

For both $\mathbb{C}^{n} \otimes_{\mathbb{C}} \mathbb{H}$ and $\mathbb{H}^{n}$, we use the complex structures $R_{\bar{\zeta}_{\ell}}, \ell=1,2,3$, to define the hyperkähler structure. The natural action $U(n) \curvearrowright \mathbb{C}^{n} \otimes_{\mathbb{C}} \mathbb{H}$ corresponds to the action $U(n) \subset S p(n) \curvearrowright \mathbb{H}^{n}$ induced by $M_{n}(\mathbb{C}) \subset M_{n}(\mathbb{H}) \curvearrowright \mathbb{H}^{n}$. Its moment map can be computed as follows:

Let $x=v_{1}+v_{2} j$ with $v_{1}, v_{2} \in \mathbb{C}^{n}, \zeta \in \mathfrak{s p}(1)$ and $\xi \in \mathfrak{u}(n)$. Then the moment map is

$$
\begin{aligned}
\left\langle\mu_{U(n)}\left(v_{1}+v_{2} j\right), \zeta \otimes \xi\right\rangle= & \frac{1}{2} \operatorname{Re}\left(\zeta\left(v_{1}+v_{2} j\right)^{*} \xi\left(v_{1}+v_{2} j\right)\right) \\
= & \frac{1}{2} \operatorname{Re}\left(\zeta\left(v_{1}^{*} \xi v_{1}-v_{2}^{*} \xi v_{2}\right)\right)-\operatorname{Re}\left(\zeta j v_{2}^{*} \xi v_{1}\right) \\
= & -\frac{1}{2}\left\langle\zeta, \operatorname{tr}\left(\xi\left(v_{1} \otimes v_{1}^{*}-v_{2} \otimes v_{2}^{*}\right)\right)\right\rangle_{\mathfrak{s p}(1)}+\left\langle\zeta, j \operatorname{tr}\left(\xi v_{1} \otimes v_{2}^{*}\right)\right\rangle_{\mathfrak{s p}(1)} \\
= & \frac{1}{2}\left\langle\zeta, i \operatorname{tr}\left(\xi i\left(v_{1} \otimes v_{1}^{*}-v_{2} \otimes v_{2}^{*}\right)\right)\right\rangle_{\mathfrak{s p}(1)} \\
& +\left\langle\zeta, j \operatorname{Re}\left(\operatorname{tr}\left(\xi v_{1} \otimes v_{2}^{*}\right)\right)-k \operatorname{Im}\left(\operatorname{tr}\left(\xi v_{1} \otimes v_{2}^{*}\right)\right)\right\rangle_{\mathfrak{s p}(1)} \\
= & -\frac{1}{2 n}\left(\langle\zeta, i\rangle_{\mathfrak{s p}(1)}\left\langle\xi, i\left(v_{1} \otimes v_{1}^{*}-v_{2} \otimes v_{2}^{*}\right)\right\rangle_{\mathfrak{u}(n)}\right. \\
& +\langle\zeta, j\rangle_{\mathfrak{s p}(1)}\left\langle\xi, v_{1} \otimes v_{2}^{*}-v_{2} \otimes v_{1}^{*}\right\rangle_{\mathfrak{u}(n)} \\
& \left.+\langle\zeta, k\rangle_{\mathfrak{s p}(1)}\left\langle\xi, i\left(v_{1} \otimes v_{2}^{*}+v_{2} \otimes v_{1}^{*}\right)\right\rangle_{\mathfrak{u}(n)}\right),
\end{aligned}
$$


where $\langle A, B\rangle_{\mathfrak{u}(n)}=-n \operatorname{tr}(A B)$ for $A, B \in \mathfrak{u}(n)$. Alternatively, using $\mathfrak{s p}(1)^{\vee} \otimes \mathfrak{u}(n)^{\vee} \cong$ $\mathfrak{s p}(1) \otimes \mathfrak{u}(n)$, we have

$$
\begin{aligned}
& \mu_{U(n)}\left(v_{1}+v_{2} j\right) \\
= & -\frac{1}{2 n}\left(i \otimes i\left(v_{1} \otimes v_{1}^{*}-v_{2} \otimes v_{2}^{*}\right)+j \otimes\left(v_{1} \otimes v_{2}^{*}-v_{2} \otimes v_{1}^{*}\right)+k \otimes i\left(v_{1} \otimes v_{2}^{*}+v_{2} \otimes v_{1}^{*}\right)\right) .
\end{aligned}
$$

This is the form in which this moment map (or its restriction to Lie subalgebras of $\mathfrak{u}(n)$ ) often appears in the literature.

For $m \in \mathbb{Z}$, let us now consider the action $U(1) \rightarrow U(n) \hookrightarrow S p(n) \curvearrowright \mathbb{H}^{n}, z \mapsto z^{m}$. For $\zeta \in \mathfrak{s p}(1)$ and $i \in i \mathbb{R}=\operatorname{Lie}(U(1))$, we have

$$
\left\langle\mu_{U(1)}(x), \zeta \otimes i\right\rangle=\frac{m}{2} \operatorname{Re}\left(\zeta x^{*} i x\right)=-\frac{m}{2}\left\langle\zeta, x^{*} i x\right\rangle_{\mathfrak{s p}(1)}
$$

Alternatively, using $\mathfrak{s p}(1)^{\vee} \otimes \mathfrak{u}(1)^{\vee} \cong \mathfrak{s p}(1)$ (using evaluation at $i$ to identify $\mathfrak{u}(1)^{\vee} \cong \mathbb{R}$ ), we have

$$
\mu_{U(1)}(x)=-\frac{m}{2} x^{*} i x
$$

In terms of $v_{1}, v_{2} \in \mathbb{C}^{n}$, we have $\mu_{U(1)}=\mu_{1} i+\mu_{\mathbb{C}} j$ with

$$
\begin{aligned}
& \mu_{1}\left(v_{1}+v_{2} j\right)=-\frac{m}{2}\left(\left\|v_{1}\right\|^{2}-\left\|v_{2}\right\|^{2}\right), \\
& \mu_{\mathbb{C}}\left(v_{1}+v_{2} j\right)=-m i v_{1}^{*} v_{2} .
\end{aligned}
$$

For $m=1$, the hyperkähler quotient of $\mathbb{H}^{n}$ by this $S^{1}$-action at the level $\frac{i}{2} \in \mathfrak{s p}(1) \cong$ $\mathfrak{s p}(1)^{\vee} \otimes(i \mathbb{R})^{\vee}$ is $T^{*} \mathbb{C} P^{n-1}$ with the Calabi metric ([Cal79], [Fei99]). The quotient has a residual hamiltonian $P U(n)$-action.

Note that if we take the same action on $\mathbb{H}^{n} \backslash\{0\}$, then the hyperkähler reduction at 0 gives the highest weight nilpotent coadjoint orbit of $S L_{n}(\mathbb{C})$ (cf. [BGM93, Example 4]).

2.1.26 Example $\left(\mathbb{H}^{n} \curvearrowright \mathbb{H}^{n}\right)$. We also have $\mathbb{H}^{n}$ acting by hyperkähler isometries on $\mathbb{H}^{n}$ (Example 2.1.8) by translations: $\mathbb{H}^{n} \curvearrowright \mathbb{H}^{n},(h, x) \mapsto h+x$. The fundamental vector field is $\left.v_{h}^{\mathbb{H}^{n}}\right|_{x}=-h$. There is a hyperkähler moment map

$$
\begin{aligned}
\mu^{\mathbb{H}^{n}}: \mathbb{H}^{n} & \rightarrow \mathfrak{s p}(1)^{\vee} \otimes\left(\mathbb{H}^{n}\right)^{\vee} \\
\left\langle\mu^{\mathbb{H}^{n}}(x), h\right\rangle & =\operatorname{Im}\left(h^{*} x\right) \in \operatorname{Im}(\mathbb{H}) \cong \mathfrak{s p}(1)^{\vee} .
\end{aligned}
$$

When restricting this action to $\mathbb{R}^{n} \subset \mathbb{H}^{n}$, the moment map is $\left\langle\mu^{\mathbb{R}^{n}}(x), v\right\rangle=\operatorname{Im}\left(v^{*} x\right)=$ $v^{*} \operatorname{Im}(x)$, where $v \in \mathbb{R}^{n}$.

This action for $n=1$ can be combined with the action $\mathbb{R} \rightarrow S^{1} \curvearrowright \mathbb{H}^{m}$ to an action $\mathbb{R} \curvearrowright \mathbb{H}^{m+1}$, whose reduction at level 0 is the generalized Taub-NUT metric (cf. [BGM93, Example 3]) 
2.1.27 Remark. Note that we also have a hyperkähler action of the semidirect product $\mathbb{H}^{n} \rtimes S p(n) \curvearrowright \mathbb{H}^{n},((h, A), x) \mapsto A x+h$ for $A \in S p(n), h, x \in \mathbb{H}^{n}$. The fundamental vector field for this action is $\left.v_{(h, \xi)}^{\mathbb{H}^{n} \rtimes S p(n)}\right|_{x}=-\xi x-h$, and

$$
\begin{array}{r}
\mu^{\mathbb{H}^{n} \rtimes S p(n)}: \mathbb{H}^{n} \rightarrow\left(\mathbb{H}^{n} \rtimes \mathfrak{s p}(n)\right)^{\vee} \otimes \mathfrak{s p}(1)^{\vee}, \\
\left\langle\mu^{\mathbb{H}^{n} \rtimes S p(n)}(x),(h, \xi) \otimes \zeta\right\rangle=\frac{1}{2} \operatorname{Re}\left(\zeta\left(x^{*} \xi x-2 \operatorname{Im}\left(h^{*} x\right)\right)\right)
\end{array}
$$

is a hyperkähler moment map.

\subsubsection{Hyperkähler potential}

2.1.28 Definition (Kähler potential). Let $\left(M, g^{M}, I\right)$ be a Kähler manifold with Kähler form $\omega$. For a 1-form $\alpha \in \Omega^{1}(M)$ define $I \alpha \in \Omega^{1}(M)$ by $I \alpha(v):=\alpha(I(v))$ for all $v \in T M$. A smooth function $f: M \rightarrow \mathbb{R}$ is said to be a Kähler potential if $d I d f=2 \omega$.

2.1.29 Remark. In terms of complex valued differential forms and Dolbeault operators, this condition reads $-i \partial \bar{\partial} f=\omega$.

2.1.30 Definition (hyperkähler potential). A smooth map $f: M \rightarrow \mathbb{R}$ on a hyperkähler manifold $\left(M, g^{M}, I_{1}, I_{2}, I_{3}\right)$ is said to be a hyperkähler potential if $f$ is a Kähler potential for each of the three complex structures:

$$
d \mathcal{I}_{\zeta} d f=2 \omega_{\zeta} \text { for all } \zeta \in \mathfrak{s p}(1),\|\zeta\|^{2}=1
$$

2.1.31 Example (hyperkähler potential for $\mathbb{H}^{n}$ ). Consider the hyperkähler manifold $M=\mathbb{H}^{n}$ (with either of the hyperkähler structures from Example 2.1.7 or Example 2.1.8). Then the function $f: \mathbb{H}^{n} \rightarrow \mathbb{R}, f(h)=\frac{1}{2}\|h\|^{2}$ is a hyperkähler potential. This is easy to check and will also follow from Example 2.2.50.

\subsection{Hyperkähler manifolds with permuting actions}

2.2.1 Definition. An isometric action of $S p(1)$ on a hyperkähler manifold $M$ is said to be permuting if the induced action on the sphere of complex structures is the standard action of $S p(1) \rightarrow S O(3) \curvearrowright S^{2}$, i.e.

$$
q_{*} \mathcal{I}_{\zeta} \bar{q}_{*}=\mathcal{I}_{q \zeta \bar{q}} \text { for all } q \in S p(1), \zeta \in \operatorname{Im}(\mathbb{H}),\|\zeta\|^{2}=1
$$

Let $\operatorname{Spin}_{\varepsilon}^{G}(3):=(\operatorname{Sp}(1) \times G) / \pm 1$, where \pm 1 is the subgroup of order 2 generated by $(-1, \varepsilon)$, with central $\varepsilon \in G$ and $\varepsilon^{2}=1$. A $\operatorname{Spin}_{\varepsilon}^{G}(3)$-action on $M$ is said to be permuting if the action $S p(1) \rightarrow \operatorname{Spin}_{\varepsilon}^{G}(3) \curvearrowright M$ is permuting and the action $G \rightarrow \operatorname{Spin}_{\varepsilon}^{G}(3) \curvearrowright M$ is hyperkähler. 
2.2.2 Remark. Note that $\operatorname{Spin}(3) \cong \operatorname{Sp}(1)$ and hence, $\operatorname{Spin}_{\varepsilon}^{G}(3)$ generalizes $\operatorname{Spin}(3)$, $\operatorname{Spin}^{c}(3)$ and $S O(3) \times G$.

Since $\operatorname{Spin}(4) \cong S p(1)_{+} \times S p(1)_{-},{ }^{1}$ denote $\operatorname{Spin}_{\varepsilon}^{G}(4):=\operatorname{Spin}_{(-1, \varepsilon)}{\operatorname{Sp}(1)_{-} G}_{(3)} \cong(\operatorname{Spin}(4) \times$ $G) / \pm 1$. Therefore, a $\operatorname{Spin}_{\varepsilon}^{G}(4)$-action on $M$ is permuting if the $S p(1)_{+}$-action is permuting while the $S p(1)_{-} \times G$-action is hyperkähler.

2.2.3 Remark. Since we assume permuting actions to be isometric, we can use any of the following equivalent conditions for an isometric $S p(1)$-action on a hyperkähler manifold to be permuting:

1. The induced $S p(1)$-action on End $(T M)$ restricts to the standard action

$$
S p(1) \rightarrow S O(3) \curvearrowright S^{2} \subset \mathfrak{s p}(1) \stackrel{\mathcal{I}}{\hookrightarrow} \Gamma(M, \text { End }(T M)),
$$

i.e. $q_{*} \mathcal{I}_{\zeta} \bar{q}_{*}=\mathcal{I}_{q \zeta \bar{q}}$ for all $q \in S p(1), \zeta \in \operatorname{Im}(\mathbb{H})$.

2. The map $\omega: S^{2} \rightarrow \Omega^{2}(M), \zeta \mapsto \omega_{\zeta}$ is $S p(1)$-equivariant.

3. $\omega \in\left(\mathfrak{s p}(1)^{\vee} \otimes \Omega^{2}(M)\right)^{S p(1)}$, that is $L_{q}^{*} \omega_{\zeta}=\omega_{q^{-1} \zeta q}$ for all $q \in S p(1), \zeta \in \mathfrak{s p}(1)$.

4. $\mathcal{L}_{\mathfrak{s p}(1)} \omega=-\delta_{\mathfrak{s p}(1)} \omega$.

Also note that for none of these conditions really requires the action to be isometric. However, the conditions 1 . and 2. are only equivalent if the $S p(1)$-action is isometric.

2.2.4 Example (permuting actions on $\left.\left(\mathbb{H}^{n}, \boldsymbol{R}_{\bar{i}}, \boldsymbol{R}_{\bar{j}}, \boldsymbol{R}_{\bar{k}}\right)\right)$. There are two permuting $S p(1)$-actions on $\mathbb{H}^{n}$ (considered as a hyperkähler manifold as in Example 2.1.8):

1. $S p(1) \curvearrowright \mathbb{H}^{n}:(q, h) \mapsto h \bar{q}$

2. $S p(1) \rightarrow S O(3) \curvearrowright \mathbb{H}^{n}:(q, h) \mapsto q h \bar{q}$

Note that in the second case, $S p(1)$ acts trivially on $\mathbb{R}^{n} \subset \mathbb{H}^{n}$ and is the standard $S O(3)$ action on each $\left(\mathbb{R}^{3}\right)^{n} \cong \operatorname{Im}(\mathbb{H})^{n}$. This action is permuting for either of the hyperkähler structures from Example 2.1.7 and Example 2.1.8.

2.2.5 Remark. The two permuting actions in Example 2.2.4 are prototypical examples of permuting actions on hyperkähler manifolds. Is is also possible to consider one of these actions on some factors of $\mathbb{H}^{n}$ and the other one on the remaining factors. In many examples, a permuting action arises as a residual action on a hyperkähler quotient.

2.2.6 Example (permuting actions on $\left.\left(\mathbb{H}^{n}, \boldsymbol{L}_{i}, \boldsymbol{L}_{j}, \boldsymbol{L}_{\boldsymbol{k}}\right)\right)$. There are also two permuting actions on $\mathbb{H}^{n}$ (considered as a hyperkähler manifold as in Example 2.1.7):

1. $S p(1) \curvearrowright \mathbb{H}^{n}:(q, h) \mapsto q h$

2. $S p(1) \rightarrow S O(3) \curvearrowright \mathbb{H}^{n}:(q, h) \mapsto q h \bar{q}$

\footnotetext{
${ }^{1}$ We use the notation $S p(1)_{ \pm}$to distinguish the two factors of $\operatorname{Spin}(4)$.
} 
The first one is closely related to the spinor representations in dimensions three and four: It also commutes with any Lie subgroup $G \subset S p(n)$ acting by $\mathbb{H}$-linear isometries on $\mathbb{H}^{n}$. In particular, this includes the following example: Let $n=1, G=S^{1}$ and $\varepsilon=-1$. Define an action $\operatorname{Spin}^{c}(3)=(S p(1) \times G) / \pm 1 \curvearrowright M=\mathbb{H}$ :

$$
[(q, z)] \cdot h:=q h z \text { for }[(q, z)] \in\left(S p(1) \times S^{1}\right) / \pm 1, h \in \mathbb{H} .
$$

This is a permuting $\operatorname{Spin}_{\varepsilon}^{G}(3)$-action, which is the spinor representation $W$ of $\operatorname{Spin}^{c}(3)=$ $\left(S p(1) \times S^{1}\right) / \pm 1$. If we interpret $M=\mathbb{H}$ as a hyperkähler manifold with permuting $\operatorname{Spin}^{c}(4)$-action (with trivial $S p(1)_{-}$-action), we obtain the $\operatorname{Spin}^{c}(4)$-representation $W^{+}$. This uses the following identifications:

1. $\operatorname{Spin}(3) \cong S p(1) \subset \mathbb{H} \stackrel{\text { diag }}{\rightarrow} \mathbb{H} \oplus \mathbb{H} \cong C l_{3}$,

2. $\operatorname{Spin}(4) \cong S p(1)_{+} \times S p(1)_{-} \subset \mathbb{H} \oplus \mathbb{H} \hookrightarrow M_{2}(\mathbb{H}) \cong C l_{4}$

3. $C l_{3} \cong C l_{4}^{0} \hookrightarrow C l_{4},\left(h, h^{\prime}\right) \mapsto\left(\begin{array}{cc}h & 0 \\ 0 & h^{\prime}\end{array}\right) \in M_{2}(\mathbb{H})$

Here is a list of useful representations of $\operatorname{Spin}(3)$ and $\operatorname{Spin}^{c}(3)$ :

\begin{tabular}{|c|c|ll|}
\hline name & vector space & homomorphism & \\
\hline \hline $\mathrm{R}^{3}$ & $\mathrm{R}^{3} \cong \operatorname{Im}(\mathbb{H})$ & $S p(1) \rightarrow S O(3)$ & $q \cdot v=q v \bar{q}$ for $v \in \operatorname{Im}(\mathbb{H}) \cong \mathbb{R}^{3}$ \\
$S$ & $\mathbb{H}$ & $S p(1) \rightarrow \operatorname{Aut}(\mathbb{H})$ & $q \cdot h=q h$ for $v \in \mathbb{H}=S$ \\
$W$ & $\mathbb{H}$ & $\operatorname{Spin}^{c}(3) \rightarrow \operatorname{Aut}(\mathbb{H})$ & {$[(q, z)] \cdot h=q h z$ for $v \in \mathbb{H}$} \\
\hline
\end{tabular}

Here $q \in S p(1), z \in S^{1}$ and $[(q, z)] \in\left(S p(1) \times S^{1}\right) / \pm 1 \cong \operatorname{Spin}^{c}(3)$.

Here is a list of useful representations of $\operatorname{Spin}(4)$ and $\operatorname{Spin}^{c}(4)$ :

\begin{tabular}{|c|c|c|c|}
\hline name & vector space & homomorphism & \\
\hline $\mathrm{R}^{4}$ & $\mathrm{R}^{4} \cong \mathrm{H}$ & $\overline{S \operatorname{Spin}(4) \rightarrow S O(4)}$ & $\left(q_{+}, q_{-}\right) \cdot h=q_{+} h \bar{q}_{-}$for $h \in \mathbb{H} \cong \mathbb{R}^{4}$ \\
\hline$S^{+}$ & $\mathrm{H}$ & $\operatorname{Spin}(4) \rightarrow \operatorname{Aut}(\mathbb{H})$ & $\left(q_{+}, q_{-}\right) \cdot h=q_{+} h$ for $h \in \mathbb{H}$ \\
\hline$S^{-}$ & $\mathrm{H}$ & $\operatorname{Spin}(4) \rightarrow \operatorname{Aut}(\mathbb{H})$ & $\left(q_{+}, q_{-}\right) \cdot h=q_{-} h$ for $h \in \mathbb{H}$ \\
\hline $\mathbb{R}^{4}$ & $\mathbb{R}^{4} \cong \mathbb{H}$ & $\operatorname{Spin}^{c}(4) \rightarrow S O(4)$ & {$\left[\left(q_{+}, q_{-}, z\right)\right] \cdot h=q_{+} h \bar{q}_{-}$for $h \in \mathbb{H} \cong \mathbb{R}^{4}$} \\
\hline$W^{+}$ & $\mathrm{H}$ & $\operatorname{Spin}^{c}(4) \rightarrow \operatorname{Aut}(\mathbb{H})$ & {$\left[\left(q_{+}, q_{-}, z\right)\right] \cdot h=q_{+} h z$ for $h \in W \cong \mathbb{H}$} \\
\hline$W^{-}$ & $\mathrm{H}$ & $\operatorname{Spin}^{c}(4) \rightarrow \operatorname{Aut}(\mathbb{H})$ & {$\left[\left(q_{+}, q_{-}, z\right)\right] \cdot h=q_{-} h z$ for $h \in W \cong \mathbb{H}$} \\
\hline
\end{tabular}

\subsubsection{Differential forms from permuting actions}

We will now recall some results about differential forms on hyperkähler manifolds with permuting actions. Some of these appear in the work of Swann [Swa91], who studied the case when a hyperkähler potential exists, Boyer, Galicki, Mann [BGM93, §2], who studied $\rho$ in terms of $d \rho$ and assumed $H^{1}(M, \mathbb{R})=0$ and Pidstrygach [Pid04, Section 2.2.1], who observed that $\rho$ can be constructed explicitly in the general case.

2.2.7 Proposition. [BGM93, Pid04] Let $S p(1) \curvearrowright M$ be a permuting action on a hyperkähler manifold $M$. Then 
1. $\omega=d \gamma$, where $\gamma:=\frac{1}{2} \pi_{\mathfrak{s p}(1)^{\vee}} \iota_{\mathfrak{s p}(1)} \omega \in\left(\Omega^{1}(M) \otimes \mathfrak{s p}(1)^{\vee}\right)^{S p(1)}$,

2. $\iota_{\mathfrak{s p}(1)} \omega=-\delta_{\mathfrak{s p}(1)} \gamma+d \rho$, where $\rho:=-\iota_{\mathfrak{s p}(1)} \gamma \in\left(\Omega^{0}(M) \otimes \mathfrak{s p}(1)^{\vee} \otimes \mathfrak{s p}(1)^{\vee}\right)^{S p(1)}$,

3. $\rho$ is symmetric, i.e. $\rho \in\left(\Omega^{0}(M) \otimes S^{2}\left(\mathfrak{s p}(1)^{\vee}\right)\right)^{S p(1)}$,

4. $d \rho-\pi_{S^{2} \mathfrak{s p}(1) \vee} \iota_{\mathfrak{s p}(1)} \omega=0$.

Furthermore, if $G \curvearrowright M$ is a hyperkähler action that commutes with the permuting $S p(1)$ action, then $\mu:=\iota_{\mathfrak{g}} \gamma \in C^{\infty}\left(M, \mathfrak{g}^{\vee} \otimes \mathfrak{s p}(1)^{\vee}\right)^{S p(1) \times G}$ is a hyperkähler moment map for this action.

Here, $S^{2}\left(\mathfrak{s p}(1)^{\vee}\right)$ is the second symmetric power of the coadjoint representation $\mathfrak{s p}(1)^{\vee}$, $\pi_{\mathfrak{s p}(1)^{\vee}}: \mathfrak{s p}(1)^{\vee} \otimes \mathfrak{s p}(1)^{\vee} \rightarrow \mathfrak{s p}(1)^{\vee}$ denotes the dual of the map $\pi_{\mathfrak{s p}(1) \vee}^{\vee}: \mathfrak{s p}(1) \rightarrow \mathfrak{s p}(1) \otimes$ $\mathfrak{s p}(1), i \mapsto \frac{1}{2}(j \otimes k-k \otimes j), j \mapsto \frac{1}{2}(k \otimes i-i \otimes k), k \mapsto \frac{1}{2}(i \otimes j-j \otimes i)$, and $\pi_{S^{2} \mathfrak{s p}(1)^{\vee}}: \mathfrak{s p}(1)^{\vee} \otimes$ $\mathfrak{s p}(1)^{\vee} \rightarrow S^{2}\left(\mathfrak{s p}(1)^{\vee}\right)$ is the symmetrization $\eta_{1} \otimes \eta_{2} \mapsto \frac{1}{2}\left(\eta_{1} \otimes \eta_{2}+\eta_{2} \otimes \eta_{1}\right)$.

Proof.

1. Since $d \omega=0$ and $[\cdot, \cdot] \circ \pi_{\mathfrak{s p}(1)^{\vee}}^{\vee}=2 \mathrm{id}_{\mathfrak{s p}(1)}$, we have

$$
d \gamma=\frac{1}{2} \pi_{\mathfrak{s p}(1) \vee} d \iota_{\mathfrak{s p}(1)} \omega=\frac{1}{2} \pi_{\mathfrak{s p}(1) \vee} \mathcal{L}_{\mathfrak{s p}(1)} \omega=\frac{1}{2} \pi_{\mathfrak{s p}(1) \vee} \circ[\cdot, \cdot]^{\vee} \omega=\omega .
$$

2. $\iota_{\mathfrak{s p}(1)} \omega=\iota_{\mathfrak{s p}(1)} d \gamma=\mathcal{L}_{\mathfrak{s p}(1)} \gamma-d \iota_{\mathfrak{s p}(1)} \gamma=-\delta_{\mathfrak{s p}(1)} \gamma+d \rho$.

3. Using that $\frac{1}{2} \pi_{\mathfrak{s p}(1) \vee}^{\vee} \circ[\cdot, \cdot]=\pi_{\wedge^{2} \mathfrak{s p}(1) \vee}$ is the skew-symmetrization, we compute the skew-symmetric part of $\iota_{\mathfrak{s p}(1)} \gamma$ :

$$
\begin{aligned}
& \left(\iota_{\mathfrak{s p}(1)} \gamma\right)\left(\xi_{1} \otimes \xi_{2}-\xi_{2} \otimes \xi_{1}\right)=\frac{1}{2}\left(\iota_{\mathfrak{s p}(1)} \gamma\right)\left(\pi_{\mathfrak{s p}(1) \vee}^{\vee} \circ[\cdot, \cdot]\left(\xi_{1} \otimes \xi_{2}-\xi_{2} \otimes \xi_{1}\right)\right) \\
& =\left(\pi_{\mathfrak{s p}(1) \vee} \iota_{\mathfrak{s p}(1)} \gamma\right)\left(\left[\xi_{1}, \xi_{2}\right]\right) \\
& =\left(\mathcal{L}_{\mathfrak{s p}(1)} \pi_{\mathfrak{s p}(1) \vee} \iota_{\mathfrak{s p}(1)} \gamma\right)\left(\xi_{1} \otimes \xi_{2}\right) \\
& =\left(\iota_{\mathfrak{s p}(1)} \pi_{\mathfrak{s p}(1) \vee} d \iota_{\mathfrak{s p}(1)} \gamma\right)\left(\xi_{1} \otimes \xi_{2}\right) \\
& =\left(\iota_{\mathfrak{s p}(1)} \pi_{\mathfrak{s p}(1) \vee} \mathcal{L}_{\mathfrak{s p}(1)} \gamma\right)\left(\xi_{1} \otimes \xi_{2}\right) \\
& -\left(\iota_{\mathfrak{s p}(1)} \pi_{\mathfrak{s p}(1) \vee} \iota_{\mathfrak{s p}(1)} d \gamma\right)\left(\xi_{1} \otimes \xi_{2}\right)
\end{aligned}
$$

Observing that $\pi_{\mathfrak{s p}(1) \vee} \mathcal{L}_{\mathfrak{s p}(1)} \gamma=2 \gamma$ and $\pi_{\mathfrak{s p}(1) \vee} \iota_{\mathfrak{s p}(1)} d \gamma=\pi_{\mathfrak{s p}(1) \vee} \iota_{\mathfrak{s p}(1)} \omega=2 \gamma$, we see that the expression above vanishes.

4. To prove the last assertion, we only need to apply the symmetrization $\pi_{S^{2} \mathfrak{s p}(1) \vee}$ to the equation $\iota_{\mathfrak{s p}(1)} \omega=-\delta_{\mathfrak{s p}(1)} \gamma+d \rho$.

Note that if $G$ acts hyperkähler and the $G$-action commutes with the permuting $S p(1)$ action, the forms $\omega$ and $\gamma$ are not only $S p(1)$-invariant, but also $G$-invariant forms. Since $\iota_{\mathfrak{g}}$ preserves the invariance, $\mu=\iota_{\mathfrak{g}} \gamma \in C^{\infty}\left(M, \mathfrak{g}^{\vee} \otimes \mathfrak{s p}(1)^{\vee}\right)^{S p(1) \times G}$. The moment map condition follows immediately from Cartan's formula and the $G$-invariance of $\gamma$ :

$$
d \mu=d \iota_{\mathfrak{g}} \gamma=\mathcal{L}_{\mathfrak{g}} \mu-\iota_{\mathfrak{g}} d \gamma=-\iota_{\mathfrak{g}} \omega
$$


2.2.8 Notation. Consider the decomposition of

$$
\mathfrak{s p}(1) \otimes \mathfrak{s p}(1) \cong S^{2}(\mathfrak{s p}(1)) \oplus \bigwedge^{2} \mathfrak{s p}(1) \cong \mathbb{R} \oplus \bigwedge^{2} \mathfrak{s p}(1) \oplus S_{0}^{2}(\mathfrak{s p}(1))
$$

of $S p(1)$-representations into irreducibles. More precisely, we consider the four projections $\operatorname{pr}_{\mathbb{R}}, \operatorname{pr}_{\wedge^{2}}, \operatorname{pr}_{S^{2} \mathfrak{s p}(1)}, \operatorname{pr}_{S_{0}^{2} \mathfrak{s p}(1)} \in \operatorname{End}(\mathfrak{s p}(1) \otimes \mathfrak{s p}(1))$ :

$$
\begin{array}{r}
\operatorname{pr}_{\mathbb{R}}: \mathfrak{s p}(1) \otimes \mathfrak{s p}(1) \rightarrow \mathfrak{s p}(1) \otimes \mathfrak{s p}(1), \zeta \otimes \zeta^{\prime} \mapsto\left\langle\zeta, \zeta^{\prime}\right\rangle_{\mathbb{H}} \frac{1}{3} \sum_{\ell=1}^{3} \zeta_{\ell} \otimes \zeta_{\ell}, \\
\operatorname{pr}_{\wedge^{2}}: \mathfrak{s p}(1) \otimes \mathfrak{s p}(1) \rightarrow \mathfrak{s p}(1) \otimes \mathfrak{s p}(1), \zeta \otimes \zeta^{\prime} \mapsto \frac{1}{2}\left(\zeta \otimes \zeta^{\prime}-\zeta^{\prime} \otimes \zeta\right) . \\
\operatorname{pr}_{S^{2} \mathfrak{s p}(1)}: \mathfrak{s p}(1) \otimes \mathfrak{s p}(1) \rightarrow \mathfrak{s p}(1) \otimes \mathfrak{s p}(1), \zeta \otimes \zeta^{\prime} \mapsto \frac{1}{2}\left(\zeta \otimes \zeta^{\prime}+\zeta^{\prime} \otimes \zeta\right) . \\
\operatorname{pr}_{S_{0}^{2} \mathfrak{s p}(1)}: \mathfrak{s p}(1) \otimes \mathfrak{s p}(1) \rightarrow \mathfrak{s p}(1) \otimes \mathfrak{s p}(1), \operatorname{pr}_{S_{0}^{2} \mathfrak{s p}(1)}=\operatorname{pr}_{S^{2} \mathfrak{s p}(1)}-\operatorname{pr}_{\mathbb{R}}
\end{array}
$$

These induce the decomposition

$$
\mathfrak{s p}(1)^{\vee} \otimes \mathfrak{s p}(1)^{\vee} \cong S^{2}\left(\mathfrak{s p}(1)^{\vee}\right) \oplus \bigwedge^{2}\left(\mathfrak{s p}(1)^{\vee}\right) \cong \mathbb{R} \oplus S_{0}^{2}\left(\mathfrak{s p}(1)^{\vee}\right) \oplus \bigwedge^{2}\left(\mathfrak{s p}(1)^{\vee}\right)
$$

Therefore, we can decompose $\rho \in S^{2}\left(\mathfrak{s p}(1)^{\vee}\right) \otimes C^{\infty}(M)$ into $\rho_{0}:=-\frac{1}{3} \operatorname{tr}(\rho) \in C^{\infty}(M)$ and the traceless symmetric part $\rho_{2} \in\left(S_{0}^{2} \mathfrak{s p}(1)^{\vee} \otimes C^{\infty}(M)\right)^{S p(1)}, \rho_{2}\left(\zeta \otimes \zeta^{\prime}\right):=\rho\left(\operatorname{pr}_{S_{0}^{2} \mathfrak{s p}(1)}\left(\zeta \otimes \zeta^{\prime}\right)\right)$. Here, $\operatorname{tr}: \mathfrak{s p}(1)^{\vee} \otimes \mathfrak{s p}(1)^{\vee} \rightarrow \mathbb{R}$ is the evaluation at $i \otimes i+j \otimes j+k \otimes k$ and $S_{0}^{2}\left(\mathfrak{s p}(1)^{\vee}\right):=$ $\operatorname{ker}\left(\operatorname{tr}: S^{2}\left(\mathfrak{s p}(1)^{\vee}\right) \rightarrow \mathbb{R}\right)$.

In particular, if $\rho_{2} \equiv 0$, then $\rho_{0}$ is a hyperkähler potential (cf. $\S 2$ in [BGM93] or Corollary 2.2.37 below).

2.2.9 Remark. Note that if $G \curvearrowright M$ is a smooth hyperkähler action that commutes with the $S p(1)$-action, then all the differential forms that appear in the proposition are $G$-invariant. In particular, this holds in the case of permuting $\operatorname{Spin}_{\varepsilon}^{G}(m)$-actions: $\omega, \gamma$ and $\mu$ are $\operatorname{Spin}_{\varepsilon}^{G}(m)$-invariant.

2.2.10 Remark. The first assertion of the previous proposition implies that a hyperkähler manifold $M(\operatorname{dim}(M)>0)$ with permuting $S p(1)$-action cannot be compact ([BGM93, Prop 2.7]): For $\zeta \in \mathfrak{s p}(1),\|\zeta\|^{2}=1$, the form $\omega_{\zeta}$ is a Kähler form and exact. Therefore, the volume form is also exact, and hence $M$ cannot be compact.

\subsubsection{Example (Explicit formulae for $\gamma, \rho, \rho_{0}$ and $\rho_{2}$ ).}

We give explicit formulae for $\gamma, \rho_{0}$ and $\rho_{2}$ in terms of the Kähler forms $\omega_{1}, \omega_{2}, \omega_{3}$ and the fundamental vector fields $v_{\zeta_{\ell}}^{S p(1)}$, where $\zeta_{1}:=i, \zeta_{2}:=j, \zeta_{3}:=k$.

1. Unwrapping the definition of $\gamma$, we have

$$
\begin{aligned}
& \langle\gamma, i\rangle=\frac{1}{4}\left\langle\iota_{\mathfrak{s p}(1)} \omega, j \otimes k-k \otimes j\right\rangle=\frac{1}{4}\left(\iota_{v_{\zeta_{2}}^{S p(1)}} \omega_{3}-\iota_{v_{\zeta_{3}} p(1)} \omega_{2}\right), \\
& \langle\gamma, j\rangle=\frac{1}{4}\left\langle\iota_{\mathfrak{s p}(1)} \omega, k \otimes i-i \otimes k\right\rangle=\frac{1}{4}\left(\iota_{v_{\zeta_{3}}^{S p(1)}} \omega_{1}-\iota_{v_{\zeta_{1}}^{S p(1)}} \omega_{3}\right), \\
& \langle\gamma, k\rangle=\frac{1}{4}\left\langle\iota_{\mathfrak{s p}(1)} \omega, i \otimes j-j \otimes i\right\rangle=\frac{1}{4}\left(\iota_{v_{\zeta_{1}}^{S p(1)}} \omega_{2}-\iota_{v_{\zeta_{2}}^{S p(1)}} \omega_{1}\right) .
\end{aligned}
$$


2. Using $\rho=-\iota_{\mathfrak{s p}(1)} \gamma=-\frac{1}{2} \iota_{\mathfrak{s p}(1) \vee} \pi_{\mathfrak{s p}(1) \vee} \iota_{\mathfrak{s p}(1)} \omega=-\frac{1}{2}\left(\mathrm{id} \otimes \pi_{\mathfrak{s p}(1) \vee}\right) \iota_{\mathfrak{s p}(1)} \iota_{\mathfrak{s p}(1)} \omega$, we obtain

$$
\begin{aligned}
\langle\rho, i \otimes i\rangle & =\frac{1}{4}\left(\omega_{2}\left(v_{\zeta_{3}}^{S p(1)}, v_{\zeta_{1}}^{S p(1)}\right)-\omega_{3}\left(v_{\zeta_{2}}^{S p(1)}, v_{\zeta_{1}}^{S p(1)}\right)\right), \\
\langle\rho, j \otimes j\rangle & =\frac{1}{4}\left(\omega_{3}\left(v_{\zeta_{1}}^{S p(1)}, v_{\zeta_{2}}^{S p(1)}\right)-\omega_{1}\left(v_{\zeta_{3}}^{S p(1)}, v_{\zeta_{2}}^{S p(1)}\right)\right), \\
\langle\rho, k \otimes k\rangle & =\frac{1}{4}\left(\omega_{1}\left(v_{\zeta_{2}}^{S p(1)}, v_{\zeta_{3}}^{S p(1)}\right)-\omega_{2}\left(v_{\zeta_{1}}^{S p(1)}, v_{\zeta_{3}}^{S p(1)}\right)\right), \\
\langle\rho, i \otimes j\rangle & =\langle\rho, j \otimes i\rangle=\left\langle\rho_{2}, i \otimes j\right\rangle=\left\langle\rho_{2}, j \otimes i\right\rangle=-\frac{1}{4} \omega_{1}\left(v_{\zeta_{3}}, v_{\zeta_{1}}\right)=\frac{1}{4} \omega_{2}\left(v_{\zeta_{3}}, v_{\zeta_{2}}\right), \\
\langle\rho, j \otimes k\rangle & =\langle\rho, k \otimes j\rangle=\left\langle\rho_{2}, j \otimes k\right\rangle=\left\langle\rho_{2}, k \otimes j\right\rangle=-\frac{1}{4} \omega_{2}\left(v_{\zeta_{1}}, v_{\zeta_{2}}\right)=\frac{1}{4} \omega_{3}\left(v_{\zeta_{1}}, v_{\zeta_{3}}\right), \\
\langle\rho, k \otimes i\rangle & =\langle\rho, i \otimes k\rangle=\left\langle\rho_{2}, k \otimes i\right\rangle=\left\langle\rho_{2}, i \otimes k\right\rangle=-\frac{1}{4} \omega_{3}\left(v_{\zeta_{2}}, v_{\zeta_{3}}\right)=\frac{1}{4} \omega_{1}\left(v_{\zeta_{2}}, v_{\zeta_{1}}\right) .
\end{aligned}
$$

In particular,

$$
\begin{aligned}
& \omega_{1}\left(v_{\zeta_{3}}^{S p(1)}, v_{\zeta_{2}}^{S p(1)}\right)=2(\langle\rho, i \otimes i\rangle-\langle\rho, j \otimes j\rangle-\langle\rho, k \otimes k\rangle), \\
& \omega_{2}\left(v_{\zeta_{1}}^{S p(1)}, v_{\zeta_{3}}^{S p(1)}\right)=2(\langle\rho, j \otimes j\rangle-\langle\rho, i \otimes i\rangle-\langle\rho, k \otimes k\rangle), \\
& \omega_{3}\left(v_{\zeta_{2}}^{S p(1)}, v_{\zeta_{1}}^{S p(1)}\right)=2(\langle\rho, k \otimes k\rangle-\langle\rho, i \otimes i\rangle-\langle\rho, j \otimes j\rangle) .
\end{aligned}
$$

3. $\rho_{0}=-\frac{1}{3} \operatorname{tr}(\rho)$ yields

$$
\begin{aligned}
\rho_{0} & =\frac{1}{6}\left(g\left(I_{1} v_{\zeta_{2}}^{S p(1)}, v_{\zeta_{3}}^{S p(1)}\right)+g\left(I_{2} v_{\zeta_{3}}^{S p(1)}, v_{\zeta_{1}}^{S p(1)}\right)+g\left(I_{3} v_{\zeta_{1}}^{S p(1)}, v_{\zeta_{2}}^{S p(1)}\right),\right. \\
& =-\frac{1}{6}\left(\omega_{1}\left(v_{\zeta_{2}}^{S p(1)}, v_{\zeta_{3}}^{S p(1)}\right)+\omega_{2}\left(v_{\zeta_{3}}^{S p(1)}, v_{\zeta_{1}}^{S p(1)}\right)+\omega_{3}\left(v_{\zeta_{1}}^{S p(1)}, v_{\zeta_{2}}^{S p(1)}\right)\right),
\end{aligned}
$$

and, finally

$$
\begin{gathered}
\left\langle\rho_{2}, i \otimes i\right\rangle=-\frac{1}{6} \omega_{1}\left(v_{\zeta_{2}}^{S p(1)}, v_{\zeta_{3}}^{S p(1)}\right)+\frac{1}{12} \omega_{2}\left(v_{\zeta_{3}}^{S p(1)}, v_{\zeta_{1}}^{S p(1)}\right)+\frac{1}{12} \omega_{3}\left(v_{\zeta_{1}}^{S p(1)}, v_{\zeta_{2}}^{S p(1)}\right), \\
\left\langle\rho_{2}, j \otimes j\right\rangle=\frac{1}{12} \omega_{1}\left(v_{\zeta_{2}}^{S p(1)}, v_{\zeta_{3}}^{S p(1)}\right)-\frac{1}{6} \omega_{2}\left(v_{\zeta_{3}}^{S p(1)}, v_{\zeta_{1}}^{S p(1)}\right)+\frac{1}{12} \omega_{3}\left(v_{\zeta_{1}}^{S p(1)}, v_{\zeta_{2}}^{S p(1)}\right), \\
\left\langle\rho_{2}, k \otimes k\right\rangle=\frac{1}{12} \omega_{1}\left(v_{\zeta_{2}}^{S p(1)}, v_{\zeta_{3}}^{S p(1)}\right)+\frac{1}{12} \omega_{2}\left(v_{\zeta_{3}}^{S p(1)}, v_{\zeta_{1}}^{S p(1)}\right)-\frac{1}{6} \omega_{3}\left(v_{\zeta_{1}}^{S p(1)}, v_{\zeta_{2}}^{S p(1)}\right) .
\end{gathered}
$$

2.2.12 Example $\left(\boldsymbol{S p}(\mathbf{1}) \curvearrowright\left(\mathbb{H}^{n}, \boldsymbol{R}_{\bar{i}}, \boldsymbol{R}_{\bar{j}}, \boldsymbol{R}_{\bar{k}}\right)\right)$. Consider $\mathbb{H}^{n}$ as a hyperkähler manifold as in Example 2.1.8 and the first permuting $S p(1)$-action from Example 2.2.4. Then

$$
\begin{aligned}
& \langle\gamma, i\rangle=\frac{1}{2}\left(h_{0}^{t} d h_{1}-h_{1}^{t} d h_{0}-h_{2}^{t} d h_{3}+h_{3}^{t} d h_{2}\right), \\
& \langle\gamma, j\rangle=\frac{1}{2}\left(h_{0}^{t} d h_{2}-h_{2}^{t} d h_{0}+h_{1}^{t} d h_{3}-h_{3}^{t} d h_{1}\right), \\
& \langle\gamma, k\rangle=\frac{1}{2}\left(h_{0}^{t} d h_{3}-h_{3}^{t} d h_{0}-h_{1}^{t} d h_{2}+h_{2}^{t} d h_{1}\right),
\end{aligned}
$$

and

$$
\begin{aligned}
& \langle\rho(h), i \otimes j\rangle=\langle\rho(h), j \otimes k\rangle=\langle\rho(h), k \otimes i\rangle=0, \\
& \langle\rho(h), i \otimes i\rangle=\langle\rho(h), j \otimes j\rangle=\langle\rho(h), k \otimes k\rangle=-\rho_{0}(h)=-\frac{1}{2}\|h\|^{2} .
\end{aligned}
$$

In particular, $\rho_{2}=0$. 


\subsubsection{Modifying a permuting action}

The aim of this section is to give explicit formulae for $\rho$ in the case of the permuting $S p(1)$-action on $\mathbb{H}^{n}$ which factors through $S O(3)$, cf. Example 2.2.4. Note that this action is a diagonal action for a $S p(1) \times S p(1)$-action, where the action of one factor is permuting while the other factor preserves the hyperkähler structure.

More generally, consider a hyperkähler manifold with a permuting action $\varphi$ of $S p(1)$ and a hyperkähler action $\varphi^{h k}$ of $S p(1)$ that commutes with the permuting one. Then we can define a new diagonal $S p(1)$-action $\varphi^{\prime}$ :

$$
\varphi_{q}^{\prime}(m):=\varphi_{q}\left(\varphi_{q}^{h k}(m)\right)=\varphi_{q}^{h k}\left(\varphi_{q}(m)\right) \text { for } q \in S p(1), m \in M .
$$

Note that this action is again permuting:

$$
\left(\varphi_{q}^{\prime}\right)_{*} \mathcal{I}_{\zeta}\left(\varphi_{\bar{q}}^{\prime}\right)_{*}=\left(\varphi_{q}\right)_{*}\left(\varphi_{q}^{h k}\right)_{*} \mathcal{I}_{\zeta}\left(\varphi^{h k}\right)_{*}\left(\varphi_{\bar{q}}\right)_{*}=\left(\varphi_{q}\right)_{*} \mathcal{I}_{\zeta}\left(\varphi_{\bar{q}}\right)_{*}=\mathcal{I}_{q \zeta \bar{q}}
$$

2.2.13 Example. In the case of the first permuting $S p(1)$-action on $\mathbb{H}^{n}$ from Example 2.2.4, $\varphi_{q}(h)=h \bar{q}$, the conjugation induces the hyperkähler action $\varphi_{q}^{h k}(h)=\overline{\bar{h}} \bar{q}=q h$. The modified permuting action is the second permuting action in Example 2.2.4: $\varphi_{q}^{\prime}(h)=$ $q h \bar{q}$.

Similarly, we could have modified the permuting action only on some of the factors of $\mathbb{H}^{n}$.

The following Lemma shows how the forms $\gamma^{\prime}$ and $\rho^{\prime}$ for the modified permuting action can be computed from $\gamma$ and $\rho$ for the original permuting action.

2.2.14 Lemma. Let $M$ be a hyperkähler manifold with a $S p(1) \times S p(1)$-action, such that one $S p(1)$-action is permuting, while the other is hyperkähler. Let $\gamma, \rho, \rho_{0}, \rho_{2}$ be the forms defined in Proposition 2.2.7 for the permuting $\operatorname{Sp}(1)$-action $\varphi$, and $\mu^{h k}=\iota_{\mathfrak{s p}(1)^{h k} \gamma}$ the moment map for the hyperkähler action. Then the forms $\gamma^{\prime}, \rho^{\prime}, \rho_{0}^{\prime}, \rho_{2}^{\prime}$ for the modified permuting action are:

$$
\begin{aligned}
& \text { 1. } \gamma^{\prime}=\gamma-\frac{1}{2} \pi_{\mathfrak{s p}(1) \vee} d \mu^{h k}, \\
& \text { 2. } \rho^{\prime}=\rho-\pi_{S^{2} \mathfrak{s p}(1) \vee} \mu^{h k} \\
& \text { 3. } \rho_{0}^{\prime}=\rho_{0}+\frac{1}{3} \operatorname{tr}\left(\mu^{h k}\right) \\
& \text { 4. } \rho_{2}^{\prime}=\rho_{2}-\pi_{S_{0}^{2} \mathfrak{s p}(1) \vee} \mu^{h k}
\end{aligned}
$$

Proof. Since the two actions commute, the fundamental vector field for the modified action is given as follows:

$$
\left.v_{\zeta}^{S p(1)^{\prime}}\right|_{m}=\left.v_{\zeta}^{S p(1)}\right|_{m}+\left.v_{\zeta}^{S p(1)^{h k}}\right|_{m}
$$

In particular, $\iota_{\mathfrak{s p}(1)^{\prime}}=\iota_{\mathfrak{s p}(1)}+\iota_{\mathfrak{s p}(1)^{h k}}$.

1. $\gamma^{\prime}=\frac{1}{2} \pi_{\mathfrak{s p}(1)^{\vee}} \iota_{\mathfrak{s p}(1)^{\prime}} \omega=\frac{1}{2} \pi_{\mathfrak{s p}(1)^{\vee}} \iota_{\mathfrak{s p}(1)} \omega+\frac{1}{2} \pi_{\mathfrak{s p}(1)^{\vee}} \iota_{\mathfrak{s p}(1)^{h k}} \omega=\gamma-\frac{1}{2} \pi_{\mathfrak{s p}(1)^{\vee}} d \mu^{h k}$. 
2. Using the fact that $\pi_{\mathfrak{s p}(1)^{\vee}} \mu^{h k}$ is $S p(1)^{\prime}$-equivariant, we compute

$$
\begin{aligned}
\rho^{\prime} & =-\iota_{\mathfrak{s p}(1)^{\prime}} \gamma^{\prime}=-\iota_{\mathfrak{s p}(1)^{\prime}}\left(\gamma-\frac{1}{2} \pi_{\mathfrak{s p}(1)^{\vee}} d \mu^{h k}\right) \\
& =-\left(\iota_{\mathfrak{s p}(1)}+\iota_{\mathfrak{s p}(1)^{h k}}\right) \gamma+\frac{1}{2} \iota_{\mathfrak{s p}(1)^{\prime}} \pi_{\mathfrak{s p}(1)^{\vee}} d \mu^{h k} \\
& =\rho-\mu^{h k}+\frac{1}{2} \mathcal{L}_{\mathfrak{s p}(1)^{\prime}} \pi_{\mathfrak{s p}(1)^{\vee}} \mu^{h k} \\
& =\rho-\mu^{h k}-\frac{1}{2}[\cdot, \cdot]^{\vee} \pi_{\mathfrak{s p}(1)^{\vee}} \mu^{h k} \\
& =\rho-\mu^{h k}+\pi_{\wedge^{2} \mathfrak{s p}(1)^{\vee}} \mu^{h k} \\
& =\rho-\pi_{S^{2} \mathfrak{s p}(1) \vee} \mu^{h k}
\end{aligned}
$$

3.\&4. These follow immediately from 2 .

2.2.15 Example $\left(\boldsymbol{S p}(\mathbf{1}) \curvearrowright\left(\mathbb{H}^{n}, \boldsymbol{R}_{-i}, \boldsymbol{R}_{-j}, \boldsymbol{R}_{-k}\right)\right)$. Using Lemma 2.2.14, we compute $\rho^{\prime}$ for the second permuting action in Example 2.2.4. the modified action. In the situation, the components of the moment map for $S p(1) \subset S p(n) \curvearrowright \mathbb{H}^{n}$ are (from Example 2.1.23):

$$
\begin{aligned}
\left\langle\mu^{h k}(x), i \otimes i\right\rangle & =\frac{1}{2}\left(-\left\|x_{0}\right\|^{2}-\left\|x_{1}\right\|^{2}+\left\|x_{2}\right\|^{2}+\left\|x_{3}\right\|^{2}\right), \\
\left\langle\mu^{h k}(x), j \otimes j\right\rangle & =\frac{1}{2}\left(-\left\|x_{0}\right\|^{2}+\left\|x_{1}\right\|^{2}-\left\|x_{2}\right\|^{2}+\left\|x_{3}\right\|^{2}\right), \\
\left\langle\mu^{h k}(x), k \otimes k\right\rangle & =\frac{1}{2}\left(-\left\|x_{0}\right\|^{2}+\left\|x_{1}\right\|^{2}+\left\|x_{2}\right\|^{2}-\left\|x_{3}\right\|^{2}\right), \\
\left\langle\mu^{h k}(x), i \otimes j\right\rangle & =-\left\langle x_{0}, x_{3}\right\rangle-\left\langle x_{1}, x_{2}\right\rangle, \\
\left\langle\mu^{h k}(x), j \otimes i\right\rangle & =\left\langle x_{0}, x_{3}\right\rangle-\left\langle x_{1}, x_{2}\right\rangle, \\
\left\langle\mu^{h k}(x), j \otimes k\right\rangle & =-\left\langle x_{0}, x_{1}\right\rangle-\left\langle x_{2}, x_{3}\right\rangle, \\
\left\langle\mu^{h k}(x), k \otimes j\right\rangle & =\left\langle x_{0}, x_{1}\right\rangle-\left\langle x_{2}, x_{3}\right\rangle, \\
\left\langle\mu^{h k}(x), k \otimes i\right\rangle & =-\left\langle x_{0}, x_{2}\right\rangle-\left\langle x_{3}, x_{1}\right\rangle, \\
\left\langle\mu^{h k}(x), i \otimes k\right\rangle & =\left\langle x_{0}, x_{2}\right\rangle-\left\langle x_{1}, x_{3}\right\rangle, \\
\left\langle\pi_{S^{2} \mathfrak{s p}(1) \vee} \mu^{h k}(x), i \otimes j\right\rangle & =-\left\langle x_{1}, x_{2}\right\rangle, \\
\left\langle\pi_{S^{2} \mathfrak{s p}(1) \vee} \mu^{h k}(x), j \otimes k\right\rangle & =-\left\langle x_{2}, x_{3}\right\rangle, \\
\left\langle\pi_{S^{2} \mathfrak{s p}(1) \vee} \mu^{h k}(x), k \otimes i\right\rangle & =-\left\langle x_{3}, x_{1}\right\rangle, \\
\operatorname{tr} \mu^{h k}(x) & =\frac{1}{2}\|\operatorname{Im}(x)\|^{2}-\frac{3}{2}\|\operatorname{Re}(x)\|^{2} .
\end{aligned}
$$

Therefore, we obtain

$$
\begin{aligned}
\rho_{0}^{\prime}(x) & =\frac{2}{3}\|\operatorname{Im}(x)\|^{2}, \\
\rho^{\prime}(i \otimes i) & =-\left\|x_{2}\right\|^{2}-\left\|x_{3}\right\|^{2}, \\
\rho^{\prime}(j \otimes j) & =-\left\|x_{1}\right\|^{2}-\left\|x_{3}\right\|^{2}, \\
\rho^{\prime}(k \otimes k) & =-\left\|x_{1}\right\|^{2}-\left\|x_{2}\right\|^{2}, \\
\rho_{2}^{\prime}(i \otimes i) & =\frac{2}{3}\left\|x_{1}\right\|^{2}-\frac{1}{3}\left\|x_{2}\right\|^{2}-\frac{1}{3}\left\|x_{3}\right\|^{2}, \\
\rho_{2}^{\prime}(j \otimes j) & =\frac{2}{3}\left\|x_{2}\right\|^{2}-\frac{1}{3}\left\|x_{1}\right\|^{2}-\frac{1}{3}\left\|x_{3}\right\|^{2}, \\
\rho_{2}^{\prime}(k \otimes k) & =\frac{2}{3}\left\|x_{3}\right\|^{2}-\frac{1}{3}\left\|x_{1}\right\|^{2}-\frac{1}{3}\left\|x_{2}\right\|^{2}, \\
\rho_{2}^{\prime}(i \otimes j) & =\rho^{\prime}(i \otimes j)=\left\langle x_{1}, x_{2}\right\rangle, \\
\rho_{2}^{\prime}(j \otimes k) & =\rho^{\prime}(j \otimes k)=\left\langle x_{2}, x_{3}\right\rangle, \\
\rho_{2}^{\prime}(k \otimes i) & =\rho^{\prime}(k \otimes i)=\left\langle x_{3}, x_{1}\right\rangle .
\end{aligned}
$$


Note that $\rho_{0}^{\prime}$ is not a hyperkähler potential. However, a hyperkähler potential exists: $x \mapsto \frac{1}{2}\|x\|^{2}$.

Similarly, one could modify the permuting action on some of the factors of $\mathbb{H}^{n}$.

\subsubsection{Permuting actions and the Cartan model}

In this section, we explain how $\omega, \gamma, \rho$ and $\mu$ can used to construct cocycles in the Cartan model for equivariant cohomology.

2.2.16 Remark. Recall that for a $G$-manifold $M$, the Cartan complex ([GS99, section $6.5])$ is

$$
\begin{aligned}
C_{G}^{*}(M) & :=\left(S^{*}\left(\mathfrak{g}^{\vee}\right) \otimes \Omega^{*}(M)\right)^{G}, \\
d_{G} & :=d-\pi_{S^{*}\left(\mathfrak{g}^{\vee}\right)} \circ \iota_{\mathfrak{g}}
\end{aligned}
$$

where $\pi_{S^{*}\left(\mathfrak{g}^{\vee}\right)} \circ \iota_{\mathfrak{g}}$ is the composition of $\iota_{\mathfrak{g}}$ and the symmetrization $\pi_{S^{*}\left(\mathfrak{g}^{\vee}\right)}: \mathfrak{g}^{\vee} \otimes S^{*}\left(\mathfrak{g}^{\vee}\right) \rightarrow$ $S^{*+1}\left(\mathfrak{g}^{\vee}\right)$. Here, $S^{*}\left(\mathfrak{g}^{\vee}\right)$ is the symmetric algebra on $\mathfrak{g}^{\vee}$. Note that the grading on $C_{G}^{*}(M)$ is given in such a way that $\mathfrak{g}^{\vee}$ is in degree 2. A detailed account of the grading will be given in subsection 3.3.5.

If $G$ is compact, then the cohomology of $C_{G}(M)$ is the equivariant cohomology of $M$ (Cartan's theorem, [Car51]).

By an observation of Atiyah and Bott ([AB84]), $\mu \in\left(\mathfrak{g}^{\vee} \otimes \Omega^{0}(M)\right)^{G}$ is a moment map for a symplectic $G$-action on $(M, \omega)$ if and only if $\omega-\mu \in C_{G}^{2}(M)$ is a cocycle in the Cartan model, i.e.

$$
0=d_{G}(\omega-\mu)=-\iota_{\mathfrak{g}} \omega-d \mu .
$$

Similarly, $\mu \in\left(\mathfrak{g}^{\vee} \otimes \mathfrak{s p}(1)^{\vee} \otimes \Omega^{0}(M)\right)^{G}$ is a moment map for a hyperkähler $G$-action on $M$ if and only if $\omega-\mu \in \mathfrak{s p}(1)^{\vee} \otimes C_{G}^{2}(M)$ is a cocycle (cf. Remark 2.1.21).

In particular, if $\omega=d \gamma$ is exact, with $\gamma \in\left(\mathfrak{s p}(1)^{\vee} \otimes \Omega^{1}(M)\right)^{G}$, then $\mu:=\iota_{\mathfrak{g}} \gamma$ is a moment map (since $\left.d_{G} \gamma=\omega-\iota_{\mathfrak{g}} \gamma\right)$.

If $S p(1) \curvearrowright M$ is permuting, then $\omega$ is an exact diffential form with natural primitive $\gamma$, and therefore, we obtain a moment map $\mu=\iota_{\mathfrak{g}} \gamma$ for any hyperkähler $G$-action, recovering the last statement of Proposition 2.2.7.

On the other hand, we can interpret $\omega \in\left(\mathfrak{s p}(1)^{\vee} \otimes \Omega^{2}(M)\right)^{S p(1)} \subset C_{S p(1)}^{4}(M)$ and $\gamma \in\left(\mathfrak{s p}(1)^{\vee} \otimes \Omega^{1}(M)\right)^{S p(1)} \subset C_{S p(1)}^{3}(M)$. We can again consider the coboundary $d_{S p(1)} \gamma$. By Proposition 2.2.7, we have

$$
d_{S p(1)} \gamma=\omega+\rho
$$

Therefore, $-\rho$ can be seen as an analogue of a moment map for a permuting action: both define cocyles $\omega-\mu \in C_{G}^{2}(M) \otimes \mathfrak{s p}(1)^{\vee}$ and $\omega+\rho \in C_{S p(1)}^{4}(M)$.

Given a $S p(1) \times G$-action on $M$, with permuting $S p(1)$-action and hyperkähler $G$-action, we also have

$$
d_{S p(1) \times G} \gamma=\omega+\rho-\mu \in C_{S p(1) \times G}^{4}(M) .
$$


Apart from the $\mathfrak{s p}(1)^{\vee}$-valued 2-form $\omega$, which can be extented to cocycles in the Cartan model, we also have a closed 4 -form $\Omega$ on any hyperkähler manifold. Theorem 2.2.22 shows how this can be extended to give 4-cocycles in the Cartan model.

2.2.17 Definition. The fundamental 4-form $\Omega$ on a hyperkähler manifold $M$ with Kähler forms $\omega_{1}, \omega_{2}, \omega_{3}$ is defined as

$$
\Omega:=\operatorname{tr}(\omega \wedge \omega)=\sum_{\ell=1}^{3} \omega_{\ell} \wedge \omega_{\ell} \in \Omega^{4}(M) .
$$

2.2.18 Remark. Since $d \omega=0$, we also have $d \Omega=0$. Furthermore, it is well-known that $\Omega$ is non-degenerate, and hence $(M, \Omega)$ is a multisymplectic (or, more precisely, 3-plectic) manifold. Indeed, for $v \in T_{x} M$ we have

$$
\begin{aligned}
& \Omega\left(v, I_{1} v, I_{2} v, I_{3} v\right) \\
= & \sum_{\ell=1}^{3} \omega_{\ell} \wedge \omega_{\ell}\left(v, I_{1} v, I_{2} v, I_{3} v\right) \\
= & 2 \sum_{\ell=1}^{3}\left(\omega_{\ell}\left(v, I_{1} v\right) \omega_{\ell}\left(I_{2} v, I_{3} v\right)-\omega_{\ell}\left(v, I_{2} v\right) \omega_{\ell}\left(I_{1} v, I_{3} v\right)+\omega_{\ell}\left(v, I_{3} v\right) \omega_{\ell}\left(I_{1} v, I_{2} v\right)\right) \\
= & 2\left(\omega_{1}\left(v, I_{1} v\right) \omega_{1}\left(I_{2} v, I_{3} v\right)-\omega_{2}\left(v, I_{2} v\right) \omega_{2}\left(I_{1} v, I_{3} v\right)+\omega_{3}\left(v, I_{3} v\right) \omega_{3}\left(I_{1} v, I_{2} v\right)\right) \\
= & 6\|v\|^{4}
\end{aligned}
$$

Therefore, $\iota_{v} \Omega \neq 0$ for all $v \neq 0$.

2.2.19 Remark. Note that by studying the fundamental 4 -form $\Omega$, we consider the hyperkähler $M$ as a quaternionic Kähler manifold (i.e. a manifold with holonomy in $S p(1) S p(n))$. Even though the complex structures are not globally defined for quaternionic Kähler manifold, $\Omega$ is still globally defined, parallel with respect to the Levi-Civita connection (and therefore closed) and non-degenerate.

2.2.20 Remark. The fundamental 4-form $\Omega$ on a hyperkähler manifold with permuting $S p(1)$-action is $S p(1)$-invariant, since tr: $\mathfrak{s p}(1)^{\vee} \otimes \mathfrak{s p}(1)^{\vee} \rightarrow \mathbb{R}$ is a morphism of $S p(1)$ representations and $\omega$ is $S p(1)$-invariant.

Before constructing the explicit 4-cocycles extending $\Omega$, we prove the following technical Lemma:

2.2.21 Lemma. Let $S p(1) \curvearrowright M$ be a permuting action on a hyperkähler manifold. Then the following equalities hold:

$$
\begin{aligned}
& \text { 1. } \iota_{\mathfrak{s p}(1)} \pi_{\mathfrak{s p}(1) \vee}(\gamma \wedge \gamma)=-2\left(\operatorname{id}_{\mathfrak{s p}(1) \vee} \otimes \pi_{\mathfrak{s p}(1) \vee}\right)(\rho \otimes \gamma) \text {, } \\
& \text { 2. } \iota_{\mathfrak{s p}(1)} \operatorname{tr}_{2,3}(\rho \otimes \omega)=\tau \operatorname{tr}_{2,4}\left(\rho \otimes\left(-\delta_{\mathfrak{s p}(1)} \gamma+d \rho\right)\right) \text {, } \\
& \text { 3. } \pi_{S^{2} \iota_{\mathfrak{s p}(1)}}\left(4 \pi_{\mathfrak{s p}(1)^{\vee}}(\gamma \wedge \gamma)+2 \operatorname{tr}_{23}(\rho \otimes \omega)\right)=d \rho^{2} \text {, }
\end{aligned}
$$


Here, $\operatorname{tr}_{i, j}:\left(\mathfrak{s p}(1)^{\vee}\right)^{\otimes k} \rightarrow\left(\mathfrak{s p}(1)^{\vee}\right)^{\otimes(k-2)}$ denotes the application of $\operatorname{tr}$ on the $i$-th and $j$-th tensor factor and the identity in all the other tensor factors. Furthermore, $\tau: \mathfrak{s p}(1)^{\vee} \otimes$ $\mathfrak{s p}(1)^{\vee} \rightarrow \mathfrak{s p}(1)^{\vee} \otimes \mathfrak{s p}(1)^{\vee}$ the dual of the map $\xi_{1} \otimes \xi_{2} \mapsto \xi_{2} \otimes \xi_{1}$ and, finally, $\rho^{2}:=\operatorname{tr}_{23}(\rho \otimes \rho)$.

Proof.

1. Use $\pi_{\mathfrak{s p}(1) \vee} \circ\left(\operatorname{id}_{\mathfrak{s p}(1)^{\vee} \otimes \mathfrak{s p}(1)^{\vee}}-\tau\right)=2 \pi_{\mathfrak{s p}(1)^{\vee}}$ to obtain

$$
\begin{aligned}
& \iota_{\mathfrak{s p}(1)} \pi_{\mathfrak{s p}(1) \vee}(\gamma \wedge \gamma)=\left(\operatorname{id}_{\mathfrak{s p}(1) \vee} \otimes \pi_{\mathfrak{s p}(1) \vee}\right) \iota_{\mathfrak{s p}(1)}(\gamma \wedge \gamma) \\
& =\left(\operatorname{id}_{\mathfrak{s p}(1) \vee} \otimes \pi_{\mathfrak{s p}(1) \vee}\right)\left(\left(\iota_{\mathfrak{s p}(1)} \gamma\right) \otimes \gamma-\left(\mathrm{id}_{\mathfrak{s p}(1) \vee} \otimes \tau\right)\left(\iota_{\mathfrak{s p}(1)} \gamma\right) \otimes \gamma\right) \\
& =2\left(\operatorname{id}_{\mathfrak{s p}(1) \vee} \otimes \pi_{\mathfrak{s p}(1) \vee}\right)\left(\left(\iota_{\mathfrak{s p}(1)} \gamma\right) \otimes \gamma\right) \\
& =-2\left(\operatorname{id}_{\mathfrak{s p}(1) \vee} \otimes \pi_{\mathfrak{s p}(1) \vee}\right)(\rho \otimes \gamma) \text {. }
\end{aligned}
$$

2. For $\xi_{1}, \xi_{2} \in \mathfrak{s p}(1)$, we have

$$
\begin{aligned}
\iota_{\mathfrak{s p}(1)} \operatorname{tr}_{2,3}(\rho \otimes \omega)\left(\xi_{1} \otimes \xi_{2}\right) & =\operatorname{tr}\left(\rho\left(\xi_{2} \otimes \cdot\right) \otimes \iota_{\xi_{\xi_{1}}} \omega\right) \\
& =\operatorname{tr}\left(\rho\left(\xi_{2} \otimes \cdot\right) \otimes\left(\gamma\left(\left[\xi_{1}, \cdot\right]\right)+d \rho\left(\xi_{1} \otimes \cdot\right)\right)\right) \\
& =\left(\operatorname{tr}_{2,4}\left(\rho \otimes\left(-\delta_{\mathfrak{s p}(1)} \gamma+d \rho\right)\right)\right)\left(\xi_{2} \otimes \xi_{1}\right) .
\end{aligned}
$$

Therefore, $\iota_{\mathfrak{s p}(1)} \operatorname{tr}_{2,3}(\rho \otimes \omega)=\tau \operatorname{tr}_{2,4}\left(\rho \otimes\left(-\delta_{\mathfrak{s p}(1)} \gamma+d \rho\right)\right)$.

3. Using $4 \pi_{\mathfrak{s p}(1)^{\vee}}=-\operatorname{tr}_{13}\left(\operatorname{id}_{\mathfrak{s p}(1)^{\vee}} \otimes \delta_{\mathfrak{s p}(1)}\right)$, we obtain

$$
\begin{aligned}
& \pi_{S^{2} \mathfrak{s p}(1) \vee} \iota_{\mathfrak{s p}(1)}\left(4 \pi_{\mathfrak{s p}(1) \vee}(\gamma \wedge \gamma)+2 \operatorname{tr}_{23}(\rho \otimes \omega)\right) \\
= & 4 \pi_{S^{2} \mathfrak{s p}(1) \vee} \iota_{\mathfrak{s p}(1)} \pi_{\mathfrak{s p}(1) \vee}(\gamma \wedge \gamma)+2 \pi_{S^{2} \mathfrak{s p}(1) \vee} \iota_{\mathfrak{s p}(1)} \operatorname{tr}_{2,3}(\rho \otimes \omega) \\
= & -8 \pi_{S^{2} \mathfrak{s p}(1) \vee}\left(\operatorname{id}_{\mathfrak{s p}(1) \vee} \otimes \pi_{\mathfrak{s p}(1) \vee}\right)(\rho \otimes \gamma)+2 \pi_{S^{2} \mathfrak{s p}(1) \vee} \operatorname{tr}_{2,4}\left(\rho \otimes\left(-\delta_{\mathfrak{s p}(1)} \gamma+d \rho\right)\right) \\
= & 2 \pi_{S^{2} \mathfrak{s p}(1) \vee} \operatorname{tr}_{2,4}\left(\rho \otimes \delta_{\mathfrak{s p}(1)} \gamma\right)-2 \pi_{S^{2} \mathfrak{s p}(1) \vee} \operatorname{tr}_{2,4}\left(\rho \otimes\left(\delta_{\mathfrak{s p}(1)} \gamma-d \rho\right)\right) \\
= & d \rho^{2} .
\end{aligned}
$$

The following theorem constructs 2-step extensions of $\Omega$ in the Cartan model $C_{H}^{*}(M)$ for $H \in\{G, S p(1), S p(1) \times G\}$. These are 4-cocycles of the form $\Omega+P_{1}^{H}+P_{2}^{H}$, where $P_{\ell}^{H} \in\left(S^{\ell} \mathfrak{h}^{\vee} \otimes \Omega^{4-2 \ell}(M)\right)^{H}$. The choices for $H$ are: a trihamiltonian $G$-action on $M$, a permuting $S p(1)$-action on $M$, or, combining the two, a permuting $S p(1) \times G$-action on $M$.

2.2.22 Theorem. Let $M$ be a hyperkähler manifold with fundamental 4-form $\Omega=$ $\operatorname{tr}(\omega \wedge \omega)$. There are the following 2-step extensions of $\Omega$ in the Cartan model for equivariant cohomology:

1. If $G \curvearrowright M$ is tri-hamiltonian with moment map $\mu, P_{1}^{G}=-2 \operatorname{tr}(\mu \otimes \omega)$ and $P_{2}^{G}:=$ $\operatorname{tr}(\mu \otimes \mu)$, then $\bar{\Omega}^{G}:=\Omega+P_{1}^{G}+P_{2}^{G}=\Omega-2 \operatorname{tr}(\mu \otimes \omega)+\operatorname{tr}(\mu \otimes \mu)=\operatorname{tr}((\omega-\mu) \wedge(\omega-\mu))$ is closed in the Cartan model for G-equivariant cohomology.

If, additionally, $\omega=d \gamma$ for some $\gamma \in \mathfrak{s p}(1)^{\vee} \otimes \Omega^{1}(M)^{G}$, there is a 1-step extension $d_{G} \operatorname{tr}(\gamma \wedge \omega)=\Omega-\operatorname{tr}(\mu \otimes \omega)-\operatorname{tr}(\gamma \wedge d \mu)$. In this case, $\bar{\Omega}^{G}=d_{G} \operatorname{tr}(\gamma \wedge(\omega-\mu))$, i.e. the two extension of $\Omega$ differ by the exact term $-d_{G} \operatorname{tr}(\gamma \otimes \mu)$. 
2. If $S p(1) \curvearrowright M$ is permuting, $P_{1}:=4 \pi_{\mathfrak{s p}(1) \vee}(\gamma \wedge \gamma)+2 \operatorname{tr}_{2,3}(\rho \otimes \omega)$ and $P_{2}:=\rho^{2}$, then $\bar{\Omega}:=\Omega+P_{1}+P_{2}=\Omega+4 \pi_{\mathfrak{s p}(1) \vee}(\gamma \wedge \gamma)+2 \operatorname{tr}_{2,3}(\rho \otimes \omega)+\rho^{2}=d_{S p(1)} \operatorname{tr}(\gamma \wedge(\omega+\rho))$ is closed in the Cartan model for Sp(1)-equivariant cohomology.

Note that $d_{S p(1)} \operatorname{tr}(\gamma \wedge \omega)=\Omega+4 \pi_{\mathfrak{s p}(1) \vee}(\gamma \wedge \gamma)+\operatorname{tr}_{13}(\gamma \wedge d \rho)+\operatorname{tr}_{23}(\rho \otimes \omega)$ is also a 1-step extension of $\Omega$. Furthermore, $\bar{\Omega}=d_{S p(1)} \operatorname{tr}(\gamma \wedge \omega)+d_{S p(1)} \operatorname{tr}_{12}(\gamma \wedge \rho)$.

3. If $S p(1) \times G \curvearrowright M$, where $S p(1)$ acts permuting while $G$ acts hyperkähler, then $\bar{\Omega}^{S p(1) \times G}:=\Omega+P_{1}+P_{1}^{G}+P_{2}+P_{2}^{G}-\operatorname{tr}_{23}(\rho \otimes \mu)=\Omega+4 \pi_{\mathfrak{s p}(1) \vee}(\gamma \wedge \gamma)+2 \operatorname{tr}_{2,3}(\rho \otimes$ $\omega)-2 \operatorname{tr}(\mu \otimes \omega)+\rho^{2}+\operatorname{tr}(\mu \otimes \mu)-\operatorname{tr}_{23}(\rho \otimes \mu)=d_{S p(1) \times G} \operatorname{tr}_{12}(\gamma \wedge(\omega-\mu+\rho))$ is closed in the Cartan model for $S p(1) \times G$-equivariant cohomology. Here, we denotes the symmetric extension of $\operatorname{tr}_{23}(\rho \otimes \mu) \in\left(\mathfrak{s p}(1)^{\vee} \otimes \mathfrak{g}^{\vee} \otimes \Omega^{0}(M)\right)^{S p(1) \times G}$ by the same name.

Note that $d_{S p(1) \times G} \operatorname{tr}(\gamma \wedge \omega)=\Omega+4 \pi_{\mathfrak{s p}(1) \vee}(\gamma \wedge \gamma)+\operatorname{tr}_{12}(\gamma \wedge d \rho)+\operatorname{tr}_{23}(\rho \otimes \omega)-$ $\operatorname{tr}(\mu \otimes \omega)+\operatorname{tr}(\gamma \wedge d \mu)$ is also a 1-step extension of $\Omega$. Furthermore, $\bar{\Omega}^{S p(1) \times G}=$ $d_{S p(1) \times G} \operatorname{tr}(\gamma \wedge \omega)+d_{S p(1) \times G} \operatorname{tr}_{12}(\gamma \wedge(\rho-\mu))$.

2.2.23 Remark. Even though in the case of a permuting action, the form $\omega$ is always exact, we still wrote down the 2-step extensions of $\Omega$ that are constructed analogously to the 2-step extension in the case of a tri-hamiltonian action. One reason for this is that the homotopy moment maps constructed from these in Proposition 3.2.3 using Theorem 3.2.1 have a simpler form than those constructed from the 1-step extension.

Furthermore, these moment maps arising from two extensions of $\Omega$ which differ by a coboundary can be thought of as "equivalent", generalizing the notion of equivalence in [FLGZ14].

2.2.24 Remark. An analogue of the first part of Theorem 2.2.22 holds for quaternionic Kähler manifolds: Let $(M, \Omega)$ be a quaternionic Kähler manifold with scalar curvature $s \neq 0$. Let $\mathcal{G}$ denote the rank 3 subbundle of almost complex structures. Denote by $\omega \in \Gamma\left(M, \mathcal{G}^{\vee} \otimes \wedge^{2} T^{*} M\right)$ the section which maps $\mathcal{G}_{x} \ni I \mapsto \omega_{I}=g(\cdot, I \cdot) \in \wedge^{2} T_{x}^{*} M$. Since this only uses the metric, $\omega$ is parallel with respect to the Levi-Civita connection $\nabla$. Let now $G \curvearrowright M$ be an action of a compact Lie group which preserves $\Omega$. Furthermore, let $\mu \in\left(\mathfrak{g}^{\vee} \otimes \Gamma\left(M, \mathcal{G}^{\vee}\right)\right)^{G}$ the corresponding moment map $\left(\nabla \mu=-\iota_{\mathfrak{g}} \omega\right.$, introduced by Galicki and Lawson in [GL88]). Since $\omega: \mathcal{G} \hookrightarrow \wedge^{2} T^{*} M$, we can use the metric on $\mathcal{G}$ to obtain an element $\operatorname{tr}_{\mathcal{G}}(\mu \otimes \omega) \in\left(\mathfrak{g}^{\vee} \otimes \Omega^{2}(M)\right)^{G}$. This satisfies $2 d \operatorname{tr}_{\mathcal{G}}(\mu \otimes \omega)=-\iota_{\mathfrak{g}} \Omega$ (cf. [Sal89, Lem. 9.7]). Using this, $\bar{\Omega}^{G}:=\Omega-2 \operatorname{tr}_{\mathcal{G}}(\mu \otimes \omega)+\operatorname{tr}_{\mathcal{G}}(\mu \otimes \mu)$ is again closed in the Cartan model for $G$-equivariant cohomology.

Proof (of Theorem 2.2.22).

1. The cocycle condition $d_{G} \bar{\Omega}^{G}=0$ is equivalent to the following three equations:

$$
\begin{aligned}
d \Omega & =0, \\
d P_{1}^{G} & =\iota_{\mathfrak{g}} \Omega, \\
d P_{2}^{G} & =\pi_{S^{2} \mathfrak{g} \vee} \iota_{\mathfrak{g}} P_{1}^{G} .
\end{aligned}
$$


The first of these follows immediately from $d \omega=0$. The second equation can be easily varified using the moment map condition $d \mu=-\iota_{\mathfrak{g}} \omega$ :

$$
d P_{1}^{G}=-2 d \operatorname{tr}(\mu \otimes \omega)=-2 \operatorname{tr}(d \mu \wedge \omega)=2 \operatorname{tr}\left(\iota_{\mathfrak{g}} \omega \wedge \omega\right)=\iota_{\mathfrak{g}} \operatorname{tr}(\omega \wedge \omega)=\iota_{\mathfrak{g}} \Omega .
$$

For the third equation, we compute for $\xi_{1}, \xi_{2} \in \mathfrak{g}$ :

$$
\begin{aligned}
d \operatorname{tr}(\mu \otimes \mu)\left(\xi_{1} \otimes \xi_{2}\right) & =d \operatorname{tr}\left(\left\langle\mu, \xi_{1}\right\rangle \otimes\left\langle\mu, \xi_{2}\right\rangle\right) \\
& =\operatorname{tr}\left(\left\langle d \mu, \xi_{1}\right\rangle \otimes\left\langle\mu, \xi_{2}\right\rangle\right)+\operatorname{tr}\left(\left\langle\mu, \xi_{1}\right\rangle \otimes\left\langle d \mu, \xi_{2}\right\rangle\right) \\
& =\operatorname{tr}(\mu \otimes d \mu)\left(\xi_{1} \otimes \xi_{2}+\xi_{2} \otimes \xi_{1}\right) \\
& =2 \pi_{S^{2} \mathfrak{g} \vee} \operatorname{tr}(\mu \otimes d \mu)\left(\xi_{1} \otimes \xi_{2}\right) \\
& =-2 \pi_{S^{2} \mathfrak{g} \vee} \operatorname{tr}\left(\mu \otimes \iota_{\mathfrak{g}} \omega\right)\left(\xi_{1} \otimes \xi_{2}\right) \\
& =\pi_{S^{2} \mathfrak{g} \vee} \iota_{\mathfrak{g}} P_{1}^{G}\left(\xi_{1} \otimes \xi_{2}\right) .
\end{aligned}
$$

If $\omega=d \gamma$, then $d \operatorname{tr}(\gamma \wedge \omega)=\Omega$, and therefore, $d_{G} \operatorname{tr}(\gamma \wedge \omega)$ is a 1-step extension of $\Omega$. Furthermore,

$$
\begin{aligned}
\bar{\Omega}^{G}-d_{G} \operatorname{tr}(\gamma \wedge \omega) & =-\operatorname{tr}(\mu \otimes \omega)+\operatorname{tr}(\gamma \wedge d \mu)+\operatorname{tr}(\mu \otimes \mu) \\
& =-d \operatorname{tr}(\mu \otimes \gamma)+\pi_{S^{2} \mathfrak{g} \vee} \iota_{\mathfrak{g}} \operatorname{tr}(\mu \otimes \gamma) \\
& =-d_{G} \operatorname{tr}(\mu \otimes \gamma)
\end{aligned}
$$

2. As in the previous case, $d_{S p(1)} \bar{\Omega}=0$ is equivalent to the following three equations:

$$
\begin{aligned}
d \Omega & =0, \\
d P_{1} & =\iota_{\mathfrak{s p}(1)} \Omega, \\
d P_{2} & =\pi_{S^{2} \mathfrak{s p}(1)} \iota_{\mathfrak{s p}(1)} P_{1} .
\end{aligned}
$$

Again, the first of these follows immediately from $d \omega=0$. The second equation is easily checked:

$$
\begin{aligned}
d P_{1} & =4 d \pi_{\mathfrak{s p}(1) \vee}(\gamma \wedge \gamma)+2 d \operatorname{tr}_{2,3}(\rho \otimes \omega) \\
& =-2 \operatorname{tr}_{2,3}\left(\delta_{\mathfrak{s p}(1)} \gamma \wedge \omega\right)+2 \operatorname{tr}_{2,3}((d \rho) \wedge \omega) \\
& =2 \operatorname{tr}_{2,3}\left(\left(-\delta_{\mathfrak{s p}(1)} \gamma+d \rho\right) \wedge \omega\right) \\
& =2 \operatorname{tr}_{2,3}\left(\left(\iota_{\mathfrak{s p}(1)} \omega\right) \wedge \omega\right) \\
& =\iota_{\mathfrak{s p}(1)} \operatorname{tr}(\omega \wedge \omega) \\
& =\iota_{\mathfrak{s p}(1)} \Omega .
\end{aligned}
$$

Here, we used $d \pi_{\mathfrak{s p}(1) \vee}(\gamma \wedge \gamma)=-\frac{1}{2} \operatorname{tr}_{2,3}\left(\delta_{\mathfrak{s p}(1)} \gamma \wedge \omega\right)$, which can easily be checked on a basis of $\mathfrak{s p}(1)$.

The third equation is the third claim in Lemma 2.2.21.

Finally, to see that this cocycle is actually $d_{S p(1)}$-exact, we compute:

$$
\begin{aligned}
& d_{S p(1)} \operatorname{tr}_{12}(\gamma \wedge(\omega+\rho)) \\
= & d \operatorname{tr}_{12}(\gamma \wedge(\omega+\rho))-\pi_{S^{*} \mathfrak{s p}(1) \vee} \iota_{\mathfrak{s p}(1)} \operatorname{tr}_{12}(\gamma \wedge(\omega+\rho)) \\
= & \operatorname{tr}_{12}(\omega \wedge(\omega+\rho))-\operatorname{tr}_{12}(\gamma \wedge d \rho)-\pi_{S^{*} \mathfrak{s p}(1)^{\vee}} \operatorname{tr}_{23}\left(\iota_{\mathfrak{s p}(1)}(\gamma \wedge(\omega+\rho))\right) \\
= & \Omega+\operatorname{tr}_{12}(\omega \otimes \rho)-\operatorname{tr}_{12}(\gamma \wedge d \rho)+\pi_{S^{*} \mathfrak{s p}(1) \vee} \operatorname{tr}_{23}(\rho \otimes(\omega+\rho))+\operatorname{tr}_{13}\left(\gamma \wedge \iota_{\mathfrak{s p}(1)} \omega\right) \\
= & \Omega+2 \operatorname{tr}_{23}(\rho \otimes \omega)-\operatorname{tr}_{12}(\gamma \wedge d \rho)+\pi_{S^{2} \mathfrak{s p}(1)^{\vee}} \operatorname{tr}_{23}(\rho \otimes \rho)+\operatorname{tr}_{13}\left(\gamma \wedge \iota_{\mathfrak{s p}(1)} \omega\right) .
\end{aligned}
$$


Since $\iota_{\mathfrak{s p}(1)} \omega=-\delta_{\mathfrak{s p}(1)} \gamma+d \rho$ and $\operatorname{tr}_{13} \circ\left(\operatorname{id}_{\mathfrak{s p}(1) \vee} \otimes \delta_{\mathfrak{s p}(1)}\right)=-4 \pi_{\mathfrak{s p}(1) \vee}$, we obtain

$$
\begin{aligned}
d_{S p(1)} \operatorname{tr}_{12}(\gamma \wedge(\omega+\rho)) & =\Omega+2 \operatorname{tr}_{23}(\rho \otimes \omega)+\pi_{S^{2} \mathfrak{s p}(1) \vee} \operatorname{tr}_{23}(\rho \otimes \rho)-\operatorname{tr}_{13}\left(\gamma \wedge \delta_{\mathfrak{s p}(1)} \gamma\right) \\
& =\Omega+2 \operatorname{tr}_{23}(\rho \otimes \omega)+\operatorname{tr}_{23}(\rho \otimes \rho)+4 \pi_{\mathfrak{s p}(1) \vee}(\gamma \wedge \gamma) .
\end{aligned}
$$

3. Using the decomposition $\operatorname{Lie}(S p(1) \times G)=\mathfrak{s p}(1) \oplus \mathfrak{g}$ and $S^{2}\left(\mathfrak{s p}(1)^{\vee} \oplus \mathfrak{g}^{\vee}\right) \cong$ $S^{2} \mathfrak{s p}(1)^{\vee} \oplus S^{2} \mathfrak{g}^{\vee} \oplus \mathfrak{s p}(1)^{\vee} \otimes \mathfrak{g}^{\vee}, d_{S p(1) \times G} \bar{\Omega}^{S p(1) \times G}=0$ is equivalent to the following equations:

$$
\begin{aligned}
d \Omega & =0, \\
d P_{1} & =\iota_{\mathfrak{s p}(1)} \Omega, \\
d P_{1}^{G} & =\iota_{\mathfrak{g}} \Omega, \\
d P_{2} & =\pi_{S^{2} \mathfrak{s p}(1)} P_{1}, \\
d P_{2}^{G} & =\pi_{S^{2} \mathfrak{g} \vee} \iota_{\mathfrak{g}} P_{1}^{G}, \\
\pi_{S^{2}\left(\mathfrak{s p}(1)^{\vee} \oplus \mathfrak{g}^{\vee}\right)}\left(\iota_{\mathfrak{s p}(1)} P_{1}^{G}+\iota_{\mathfrak{g}} P_{1}\right) & =-d \operatorname{tr}_{2,3}(\rho \otimes \mu),
\end{aligned}
$$

where, on the right hand side of the last equation, we used the symmetric extension $\mathfrak{s p}(1)^{\vee} \otimes \mathfrak{g}^{\vee} \rightarrow S^{2}\left(\mathfrak{s} \mathfrak{p}(1)^{\vee} \oplus \mathfrak{g}^{\vee}\right)$. All but the last equation follow from the previous statements. We observe that

$$
\begin{aligned}
\iota_{\mathfrak{s p}(1)} P_{1}^{G} & =-2 \iota_{\mathfrak{s p}(1)} \operatorname{tr}(\mu \otimes \omega)=2 \operatorname{tr}_{1,3}\left(\mu \otimes\left(\delta_{\mathfrak{s p}(1)} \gamma-d \rho\right)\right), \text { and } \\
\iota_{\mathfrak{g}} P_{1} & =4 \iota_{\mathfrak{g}} \pi_{\mathfrak{s p}(1) \vee}(\gamma \wedge \gamma)+2 \iota_{\mathfrak{g}} \operatorname{tr}_{2,3}(\rho \otimes \omega)=-2 \operatorname{tr}_{1,3}\left(\mu \otimes \delta_{\mathfrak{s p}(1)} \gamma\right)-2 \operatorname{tr}_{2,3}(\rho \otimes d \mu) .
\end{aligned}
$$

Here we used that $\mu$ is a moment map $\iota_{\mathfrak{g}} \gamma=\mu$ and also $2 \iota_{\mathfrak{g}} \pi_{\mathfrak{s p}(1) \vee}(\gamma \wedge \gamma)=$ $-\operatorname{tr}_{1,3}\left(\mu \otimes \delta_{\mathfrak{s p}(1)} \gamma\right)$, which follows from a short computation. Therefore, we obtain

$$
\begin{aligned}
& \pi_{S^{2}\left(\mathfrak{s p}(1) \vee \oplus \mathfrak{g}^{\vee}\right)}\left(\iota_{\mathfrak{s p}(1)} P_{1}^{G}+\iota_{\mathfrak{g}} P_{1}\right)((\zeta, 0) \otimes(0, \xi)) \\
= & -\operatorname{tr}_{1,3}(\mu \otimes d \rho)(\xi \otimes \zeta)-\operatorname{tr}_{2,3}(\rho \otimes d \mu)(\zeta \otimes \xi) \\
= & -d \operatorname{tr}_{2,3}(\rho \otimes \mu)(\zeta \otimes \xi) .
\end{aligned}
$$

This proves that the $\mathfrak{s p}(1)^{\vee} \otimes \mathfrak{g}^{\vee} \otimes \Omega^{1}(M)$-component of $d_{S p(1) \times G} \bar{\Omega}^{S p(1) \times G}=0$ holds.

Finally, we compute

$$
\begin{aligned}
& d_{S p(1) \times G} \operatorname{tr}(\gamma \wedge(\omega-\mu+\rho)) \\
= & d_{S p(1)} \operatorname{tr}(\gamma \wedge(\omega+\rho))-\pi_{S^{*}\left(\mathfrak{s p}(1)^{\vee} \oplus \mathfrak{g}^{\vee}\right)} d_{S p(1)} \operatorname{tr}(\gamma \otimes \mu) \\
& -\pi_{S^{*}\left(\mathfrak{s p}(1)^{\vee} \oplus \mathfrak{g}^{\vee}\right)_{\mathfrak{g}}} \operatorname{tr}(\gamma \wedge(\omega-\mu+\rho)) \\
= & d_{S p(1)} \operatorname{tr}(\gamma \wedge(\omega+\rho))-\operatorname{tr}(\mu \otimes \omega)+\operatorname{tr}(\gamma \wedge d \mu)-\pi_{S^{*}\left(\mathfrak{s p}(1)^{\vee} \oplus \mathfrak{g}^{\vee}\right)} \operatorname{tr}(\rho \otimes \mu) \\
& -\pi_{S^{*}\left(\mathfrak{s p}(1)^{\vee} \oplus \mathfrak{g}^{\vee}\right)} \operatorname{tr}(\mu \otimes(\omega-\mu+\rho))+\operatorname{tr}\left(\gamma \wedge \iota_{\mathfrak{g}} \omega\right) \\
= & \Omega+4 \pi_{\mathfrak{s p}(1) \vee}(\gamma \wedge \gamma)+2 \operatorname{tr}_{23}(\rho \otimes \omega)+\rho^{2}-2 \operatorname{tr}(\mu \otimes \omega)-\operatorname{tr}_{23}(\rho \otimes \mu)+\operatorname{tr}(\mu \otimes \mu) .
\end{aligned}
$$


2.2.25 Remark. Note that if $\omega$ is exact, then all the cocycles in Theorem 2.2.22 are coboundaries and, hence, the corresponding cohomology classes vanish. However, the construction of homotopy moment maps from these in Proposition 3.2.3 shows that they still contain interesting information.

Furthermore, if $\omega=d \gamma$ is exact, then $\Omega=\operatorname{tr}(\omega \wedge \omega)=d \operatorname{tr}(\gamma \wedge \omega)$ is exact and there is a 1-step extension of $\Omega$ obtained as the coboundary $d_{G} \operatorname{tr}(\gamma \wedge \omega)$.

There are, however, situations where a 1-step extension of this type cannot exist, for example if $\Omega$ (and hence $\omega$ ) is not exact.

Examples for hyperkähler manifolds with non-exact $\Omega$ are, for instance, closed hyperkähler manifolds (otherwise the volume form would be exact, and hence, by Stokes Theorem, the volume of $M$ would be zero). However, an action of a non-discrete Lie group on a closed hyperkähler manifold cannot be tri-hamiltonian, since $\mu_{2}+i \mu_{3}$ would be holomorphic with respect to $I_{1}$, and hence constant.

Non-compact examples with non-exact $\Omega$ are (certain neighborhoods of the zero section in) the cotangent bundle $T^{*} N$ of a compact real-analytic Kähler manifold $N$ ([Fei99, Thm. 2.1]): Since one of the Kähler forms, $\omega_{1}$, restricts to the Kähler form on the zero section, $\omega_{1}$ cannot be exact (nor $\omega_{1} \wedge \omega_{1}$ if $\operatorname{dim}(N)>2$ ). On the other hand, $T^{*} N$ admits a rotating $S^{1}$-action (by scalar multiplication on the fibres). Therefore, $\omega_{2}, \omega_{3}$ as well as $\omega_{2} \wedge \omega_{2}+\omega_{3} \wedge \omega_{3}$ are exact. Hence, $\Omega=\operatorname{tr}(\omega \wedge \omega)$ cannot be exact.

The most basic example of this situation is the Calabi metric on $T^{*} \mathbb{C} P^{n}$ ([Cal79]). Since it can be constructed as a hyperkähler quotient of $\mathrm{H}^{n+1}$ by $S^{1}$ (cf. [Fei99, Ex. 1.7], also Example 2.1.25), it has a residual tri-hamiltonian $P U(n+1)$-action.

\subsubsection{Vector fields on hyperkähler manifolds with permuting action}

On a hyperkähler manifold with a smooth $G$-action, we can extend (negative of) the fundamental vector field $v^{G}: \mathfrak{g} \rightarrow \Gamma(M, T M)$ to a $\mathbb{H}$-linear map $\mathfrak{g} \otimes \mathbb{H} \rightarrow \Gamma(M, T M)$ :

$$
\begin{aligned}
\mathfrak{g} \otimes \mathbb{H} & \rightarrow \Gamma(M, T M), \\
\zeta \otimes h & \mapsto-\mathcal{I}_{h} v_{\zeta}^{G} .
\end{aligned}
$$

Equivalently, we have a bundle homomorphism $\underline{\mathfrak{g} \otimes \mathbb{H}} \rightarrow T M$ from the trivial bundle to $T M$.

In particular, we are interested in the following two cases:

1. $G \curvearrowright M$ hyperkähler,

2. $S p(1) \curvearrowright M$ permuting,

and in the case when these two can be combined into an action of $\operatorname{Spin}_{\varepsilon}^{G}(m) \curvearrowright M$. 
2.2.26 Remark. Note the choice of the additional sign in the quaternionic linear extension of the fundamental vector fields. Even though this might not be the most natural choice, it is the most convenient and compatible with the existing literature.

\subsubsection{Definition (Vector fields from a permuting action).}

If the hyperkähler manifold $\left(M, g, I_{1}, I_{2}, I_{3}\right)$ comes with a permuting $\operatorname{Spin}_{\varepsilon}^{G}(3)$-action, then we have a linear map

$$
\begin{aligned}
\mathfrak{s p i n}_{\varepsilon}^{G}(3) \otimes \mathbb{H}=(\mathfrak{s p}(1) \oplus \mathfrak{g}) \otimes \mathbb{H} & \rightarrow \Gamma(M, T M), \\
(\zeta, \xi) \otimes h & \mapsto-\left(\mathcal{I}_{h} v_{\zeta}^{S p(1)}+\mathcal{I}_{h} v_{\xi}^{G}\right)
\end{aligned}
$$

We are mostly interested in the following restrictions of this map:

$$
\begin{aligned}
\chi: \mathfrak{s p}(1) \otimes \mathfrak{s p}(1) & \rightarrow \Gamma(M, T M), \zeta \otimes \zeta^{\prime} \mapsto-\mathcal{I}_{\zeta^{\prime}} v_{\zeta}^{S p(1)} \\
\mathcal{Y}: \mathfrak{g} \otimes \mathfrak{s p}(1) & \rightarrow \Gamma(M, T M), \xi \otimes \zeta \mapsto-\mathcal{I}_{\zeta} v_{\xi}^{G} \\
\chi^{G}:(\mathfrak{s p}(1) \oplus \mathfrak{g}) \otimes \mathfrak{s p}(1) & \rightarrow \Gamma(M, T M),(\zeta, \xi) \otimes \zeta^{\prime} \mapsto \chi\left(\zeta, \zeta^{\prime}\right)+\mathcal{Y}\left(\xi, \zeta^{\prime}\right) .
\end{aligned}
$$

As necessary, we will also understand $\chi \in \mathfrak{s p}(1)^{\vee} \otimes \mathfrak{s p}(1)^{\vee} \otimes \Gamma(M, T M), \chi^{G} \in \mathfrak{s p i n}_{\varepsilon}^{G}(3)^{\vee} \otimes$ $\mathfrak{s p}(1)^{\vee} \otimes \Gamma(M, T M)$ and $\mathcal{Y} \in \mathfrak{s p}(1)^{\vee} \otimes \mathfrak{g}^{\vee} \otimes \Gamma(M, T M)$.

2.2.28 Remark. Note that $\chi^{G}$ as well its components $\chi$ and $\mathcal{Y}$ are $\operatorname{Spin}_{\varepsilon}^{G}(3)$-equivariant.

\section{Components of $\chi$}

Using the decomposition $\mathfrak{s p}(1)^{\vee} \otimes \mathfrak{s p}(1)^{\vee} \cong S_{0}^{2} \mathfrak{s p}(1)^{\vee} \oplus \mathfrak{s p}(1)^{\vee} \oplus \mathbb{R}$, we decompose $\chi$ into its components. We first consider the symmetric and skew-symmetric part of $\chi$, using the isomorphism $\pi_{\mathfrak{s p}(1)^{\vee}}: \bigwedge^{2}\left(\mathfrak{s p}(1)^{\vee}\right) \cong \mathfrak{s p}(1)^{\vee}$ :

$$
\begin{aligned}
\left\langle\chi_{S y m}, \zeta \otimes \zeta^{\prime}\right\rangle & :=\left\langle\chi, \operatorname{pr}_{S^{2} \mathfrak{s p}(1)}\left(\zeta \otimes \zeta^{\prime}\right)\right\rangle=-\frac{1}{2}\left(\mathcal{I}_{\zeta} v_{\zeta^{\prime}}^{S p(1)}+\mathcal{I}_{\zeta^{\prime}} v_{\zeta}^{S p(1)}\right), \\
\chi_{A l t} & :=\pi_{\mathfrak{s p}(1)^{\vee} \chi} \chi \mathfrak{s p}(1)^{\vee} \otimes \Gamma(M, T M),
\end{aligned}
$$

and further decompose the symmetric part $\chi_{S y m} \in S^{2} \mathfrak{s p}(1)^{\vee} \otimes \Gamma(M, T M)$ into

$$
\begin{gathered}
\chi_{0}:=-\frac{1}{3} \operatorname{tr}(\chi)=\frac{1}{3} \sum_{\ell=1}^{3} I_{\ell} v_{\zeta \ell}^{S p(1)} \in \Gamma(M, T M), \\
\left\langle\chi_{2}, \zeta \otimes \zeta^{\prime}\right\rangle:=\left\langle\chi, \operatorname{pr}_{S_{0}^{2} \mathfrak{s p}(1)}\left(\zeta \otimes \zeta^{\prime}\right)\right\rangle=-\frac{1}{2}\left(\mathcal{I}_{\zeta} v_{\zeta^{\prime}}^{S p(1)}+\mathcal{I}_{\zeta^{\prime}} v_{\zeta}^{S p(1)}\right)+\operatorname{Re}\left(\zeta \overline{\zeta^{\prime}}\right) \chi_{0} .
\end{gathered}
$$

The following lemma relates the vector fields above to the differential forms on hyperkähler manifolds with permuting action in subsection 2.2.1

2.2.29 Lemma ([BGM93], [Pid04]). The following identities hold:

1. $d \rho=\iota_{\chi_{\text {Sym }}} g$ and therefore $\operatorname{grad}(\rho)=\chi_{\text {Sym }}$. In particular, $\operatorname{grad}\left(\rho_{2}\right)=\chi_{2}$ and $\operatorname{grad}\left(\rho_{0}\right)=\chi_{0}$. 
2. $\iota_{\chi A l t} g=2 \gamma$,

3. $\mu=\frac{1}{2} \iota_{\mathfrak{g}} \iota_{\chi_{A l t}} g$, i.e. $\langle\mu, \zeta \otimes \xi\rangle=\frac{1}{2} g\left(\left\langle\chi_{A l t}, \zeta\right\rangle, v_{\xi}^{G}\right)$ is a hyperkähler moment map for any hyperkähler $G$-action which commutes with the permuting Sp(1)-action.

The first two items appear in [BGM93], while the third was also observed in [Pid04] and follows from $\omega=d \gamma$. To familiarize ourselves with the notations, we quickly recall the proof:

Proof. Recall that

$$
\begin{aligned}
\rho & :=-\iota_{\mathfrak{s p}(1)} \gamma \in\left(S^{2} \mathfrak{s p}(1)^{\vee} \otimes \Omega^{0}(M)\right)^{\operatorname{Spin}_{\varepsilon}^{G}(3)}, \\
\rho_{0} & :=\frac{1}{3} \operatorname{tr}\left(\iota_{\mathfrak{s p}(1)} \gamma\right) \in \Omega^{0}(M)^{\operatorname{Spin}_{\varepsilon}^{G}(3)} \\
\rho_{2}\left(\zeta \otimes \zeta^{\prime}\right) & :=\rho\left(\operatorname{pr}_{S_{0}^{2} \mathfrak{s p}(1)}\left(\zeta \otimes \zeta^{\prime}\right)\right) \text { for } \zeta, \zeta^{\prime} \in \mathfrak{s p}(1) .
\end{aligned}
$$

1. Note that for all $\zeta, \zeta^{\prime} \in \mathfrak{s p}(1)$ we have

$$
\left\langle\iota_{\chi} g, \zeta \otimes \zeta^{\prime}\right\rangle=-g\left(\mathcal{I}_{\zeta^{\prime}} v_{\zeta}^{S p(1)}, \cdot\right)=g\left(v_{\zeta}^{S p(1)}, \mathcal{I}_{\zeta^{\prime}} \cdot\right)=\left\langle\iota_{\mathfrak{s p}(1)} \omega, \zeta \otimes \zeta^{\prime}\right\rangle
$$

The equality $\iota_{\chi_{\text {Sym }}} g=d \rho$ is obtained as the symmetrization of $\iota_{\chi} g=\iota_{\mathfrak{s p}(1)} \omega=$ $d \rho-\delta_{\mathfrak{s p}(1)} \gamma$.

2. Since $\iota_{\mathfrak{s p}(1)} \omega=\iota_{\chi} g$, we have $\gamma=\frac{1}{2} \pi_{\mathfrak{s p}(1) \vee} \iota_{\mathfrak{s p}(1)} \omega=\frac{1}{2} \pi_{\mathfrak{s p}(1) \vee} \iota_{\chi} g=\frac{1}{2} \iota_{\chi_{A l t}} g$.

3. $\mu=\iota_{\mathfrak{g}} \gamma=\frac{1}{2} \iota_{\mathfrak{g}} \iota_{\chi_{A l t}} g$.

2.2.30 Note. Note that the $\operatorname{Spin}_{\varepsilon}^{G}(3)$-invariance of $\rho_{0}$ implies the $\operatorname{Spin}_{\varepsilon}^{G}(3)$-invariance of $\chi_{0}$, i.e. $\mathcal{L}_{\mathfrak{s p i n}{ }_{\varepsilon}^{G}(3)} \chi_{0}=0$. In particular, the Lie derivative $\mathcal{L}_{\chi_{0}}$ commutes with the insertion operator $\iota_{\mathfrak{s p i n}_{\varepsilon}^{G}(3)}$.

2.2.31 Remark. For a modified rotating action as in Lemma 2.2.14, we have

$$
\chi_{0}^{\prime}=\chi_{0}+\frac{1}{3} \operatorname{grad}\left(\operatorname{tr}\left(\mu^{h k}\right)\right)
$$

\subsubsection{Rotating $S^{1}$-actions from permuting actions}

Instead of considering a permuting action of the group $S p(1)$, it is also interesting to study an action of $S^{1}$ which fixes one of the complex structures, while rotating the other two. In [Hay08], Haydys constructs another hyperkähler manifold with hyperkähler potential from such an action. A basic tool in studying such actions is the following Lemma:

2.2.32 Lemma ([HKLR87, Sec. 3.E]). Consider an isometric $S^{1}$ action on a hyperkähler manifold which preserves one of the complex structures (say $I_{1}$ ) and rotates the other two (i.e. $\mathcal{L}_{v_{i}^{S}} \omega_{1}=0, \mathcal{L}_{v_{i}^{S}} \omega_{2}=2 \omega_{3}, \mathcal{L}_{v_{i}^{S}} \omega_{3}=-2 \omega_{2}$. If $\mu^{S^{1}}: M \rightarrow(i \mathbb{R})^{\vee}$ is a moment map for this action and the symplectic form $\omega_{1}$, then $\left\langle\mu^{S^{1}}, i\right\rangle \in C^{\infty}(M, \mathbb{R})^{S^{1}}$ is a Kähler potential for $\omega_{2}$. 
Proof. The moment map condition is $d\left\langle\mu^{S^{1}}, i\right\rangle=-\iota_{v_{i}} \omega_{1}$. Therefore,

$$
d\left\langle\mu^{S^{1}}, i\right\rangle\left(I_{2}(v)\right)=-\iota_{v_{i}} \omega_{1}\left(I_{2}(v)\right)=-g\left(v_{i}^{S^{1}}, I_{1}\left(I_{2}(v)\right)\right)=-\omega_{3}\left(v_{i}^{S^{1}}, v\right)
$$

Hence $I_{2} d\left\langle\mu^{S^{1}}, i\right\rangle=-\iota_{v_{i}^{S}} \omega_{3}$. The claim now follows from

$$
d I_{2} d\left\langle\mu^{S^{1}}, i\right\rangle=-d \iota_{v_{i}^{S 1}} \omega_{3}=-\mathcal{L}_{v_{i}^{S 1}} \omega_{3}=2 \omega_{2}
$$

Thus, $\left\langle\mu^{S^{1}}, i\right\rangle$ is a Kähler potential for $\omega_{2}$.

2.2.33 Remark. $\left\langle\mu^{S^{1}}, i\right\rangle$ is a Kähler potential for any Kähler form in the circle $S^{2} \cap \omega_{1}^{\perp}$ of Kähler forms containing $\omega_{2}$ and $\omega_{3}$. Indeed, we can choose $\omega_{2}$ to be any Kähler form on this circle (and $\omega_{3}$ accordingly) and repreat the proof above.

2.2.34 Example. An example for an $S^{1}$-action as in the Lemma 2.2.32 is the following: Consider a hyperkähler manifold $M$ with permuting $S p(1)$-action and for $\zeta \in \mathfrak{s p}(1)$, $\|\zeta\|^{2}=1$ the inclusion $S^{1} \hookrightarrow S p(1), a+i b \mapsto a+\zeta b$. Then the restriction of the permuting action to $S^{1}$ satisfies the conditions

$$
\mathcal{L}_{v_{i}^{S}} \omega_{\zeta}=0, \quad \mathcal{L}_{v_{i}^{S}} \omega_{\zeta^{\prime}}=2 \omega_{\zeta^{\prime \prime}}, \quad \mathcal{L}_{v_{i}^{S}} \omega_{\zeta^{\prime \prime}}=-2 \omega_{\zeta^{\prime}}
$$

where $\left(\zeta, \zeta^{\prime}, \zeta^{\prime \prime}\right)$ are an oriented orthonormal basis is $\mathfrak{s p}(1)\left(\right.$ e.g. $\left.\zeta=i, \zeta^{\prime}=j, \zeta^{\prime \prime}=k\right)$.

The following lemma is also well-known (cf. [Sch10, Lem. 3.2.1]):

2.2.35 Lemma. Consider the $S^{1}$-action induced by the permuting action of $S p(1)$ which perserves the complex structure $\mathcal{I}_{\zeta}$. Then $\mu^{S^{1}}: M \rightarrow(i \mathbb{R})^{\vee}$ is a moment map for this $S^{1}$-action, where $\left\langle\mu^{S^{1}}, i\right\rangle=-\langle\rho, \zeta \otimes \zeta\rangle \in C^{\infty}(M, \mathbb{R})^{S^{1}}$.

Proof.

$$
d\left\langle\mu^{S^{1}}, i\right\rangle=-d\langle\rho, \zeta \otimes \zeta\rangle=-\iota_{\operatorname{grad}(\langle\rho, \zeta \otimes \zeta\rangle)} g=-\iota_{\langle\chi, \zeta \otimes \zeta\rangle} g=-\iota_{v_{\zeta}^{S p(1)}} \omega_{\zeta}
$$

We only need to observe that $v_{i}^{S^{1}}=v_{\zeta}^{S p(1)}$ since $S^{1} \subset S p(1) \curvearrowright M$.

2.2.36 Remark. This moment map can be interpreted in terms of equivariant cocycles: Given $S^{1} \hookrightarrow S p(1)$ mapping $i \mapsto \zeta$, we obtain an induced chain map

$$
C_{S p(1)}^{4}(M) \rightarrow C_{S^{1}}^{4}(M)
$$

mapping $\omega+\rho$ to $i^{\vee} \otimes\left(\omega_{\zeta}-\mu^{S^{1}}\right)$. Thus, the image in $C_{S^{1}}^{4}(M)$ is given by the product of $i^{\vee}$ and the 2-cocycle $\omega-\mu^{S^{1}}$ corresponding to the moment map $\mu^{S^{1}}$ for the $S^{1}$-action.

Since we can do this for any circle $S^{1} \hookrightarrow S p(1)$, we obtain a family of moment maps for these circle actions. In equivariant cohomology, these can be combined into the degree 4 cocycle $\omega+\rho$. 
Combining Lemma 2.2.35 and Lemma 2.2.32, we obtain the following corollary, which essentially recovers [BGM93, Thm. 2.15] (reformulated in terms of the explicitly given $\rho_{0}$ and $\rho_{2}$ which were found in $\left.[\operatorname{Pid} 04]\right)$.

2.2.37 Corollary. We obtain a map $\hat{\rho}: S^{2} \rightarrow C^{\infty}(M, \mathbb{R})$ with the following properties:

1. $\hat{\rho}(\zeta)=-\rho(\zeta \otimes \zeta)$, i.e. $-\hat{\rho}$ is the restriction to $S^{2}$ of the quadratic form associated to $\rho$.

2. $\hat{\rho}(\zeta) \in C^{\infty}(M, \mathbb{R})^{S_{\zeta}^{1}}$ is a moment map for $S_{\zeta}^{1} \curvearrowright\left(M, \omega_{\zeta}\right)$, where $S_{\zeta}^{1} \subset S p(1)$ is the stabilizer of $\omega_{\zeta}$ and we use the evaluation at $i$ as an isomorphism $(i \mathbb{R})^{\vee} \cong \mathbb{R}$.

3. $\hat{\rho}(\zeta)$ is a Kähler potential for any Kähler form in $S^{2} \cap \omega_{\zeta}^{\perp}$.

4. If $\hat{\rho}$ is constant (or equivalently $\rho_{2}=0$ ), its image $\rho_{0}$ is a hyperkähler potential.

Using the decomposition of $\rho$ into its components $\rho_{0}$ and $\rho_{2}$ (cf. Notation 2.2.8), observe that $\rho_{2}$ is the defect of the family of moment maps and Kähler potentials $-\langle\rho, \zeta \otimes \zeta\rangle$ from being independent of $\zeta$.

2.2.38 Example $\left(\boldsymbol{S}^{1}\right.$-actions and potentials for $\left.\left(\mathbb{H}^{n}, \boldsymbol{R}_{-i}, \boldsymbol{R}_{-j}, \boldsymbol{R}_{-k}\right)\right)$.

Consider $\mathbb{H}^{n}$ as a hyperkähler manifold as in Example 2.1.8. We will now consider the action of $S^{1} \subset S p(1) \curvearrowright \mathbb{H}$ which is induced by one of the two permuting actions in Example 2.2.4 and stabilizes the first complex structure:

1. Consider the following $S^{1}$-action on $\mathbb{H}^{n}:(z, h) \mapsto h \bar{z}$, where $S^{1} \subset \mathbb{C} \subset \mathbb{H}$. It follows from Example 2.2.12 that the moment map for $\omega_{1}$ and Kähler potential for $\omega_{2}, \omega_{3}$ is

$$
\left\langle\mu^{S^{1}}, i\right\rangle(h)=-\langle\rho, i \otimes i\rangle=\frac{1}{2}\|h\|^{2} .
$$

Also, $\rho_{2}=0$, and hence, $\rho_{0}(h)=\frac{1}{2}\|h\|^{2}$ is a hyperkähler potential.

2. Consider the following $S^{1}$-action on $\mathbb{H}^{n}:(z, h) \mapsto z h \bar{z}$, where $S^{1} \subset \mathbb{C} \subset \mathbb{H}$. It follows from Example 2.2.15 that the moment map for $\omega_{1}$ and Kähler potential for $\omega_{2}, \omega_{3}$ is

$$
\left\langle\mu^{S^{1}}, i\right\rangle(h)=-\langle\rho, i \otimes i\rangle=\frac{1}{2}\left(\left\|h_{2}\right\|^{2}+\left\|h_{3}\right\|^{2}\right),
$$

where $h=h_{1}+i h_{2}+j h_{3}+k h_{4}$. However, this is not a Kähler potential for $\omega_{1}$, and therefore, no hyperkähler potential, and $\rho_{2} \neq \equiv$.

A similar computation can be done for any complex structure $\mathcal{I}_{\zeta}$, not only $I_{1}$.

\subsubsection{Forms and vector fields}

The following lemma shows how the fundamental vector fields are related to the differential forms studied above:

2.2.39 Lemma. Let $M$ be a hyperkähler manifold with permuting Sp(1)-action. Then

$$
\iota_{\mathfrak{s p}(1)} g=-4 \gamma+3 \iota_{\chi_{0}} \omega \in\left(\mathfrak{s p}(1)^{\vee} \otimes \Omega^{1}(M)\right)^{S p(1)}
$$

In particular,

$$
d \iota_{\mathfrak{s p}(1)} g=3 d \mathcal{I} d \rho_{0}-4 \omega .
$$


Proof. Using the explicit formula for $\gamma$ from Example 2.2.11, we obtain

$$
\begin{aligned}
& 3\left\langle\iota_{\chi_{0}} \omega, i\right\rangle=\sum_{\ell=1}^{3} \iota_{I_{\ell} v_{\zeta_{\ell}}^{S p(1)}} \omega_{1}=\iota_{v_{\zeta_{1}}^{S p(1)}} g+\iota_{v_{\zeta_{2}}^{S p(1)}} \omega_{3}-\iota_{v_{\zeta_{3}}^{S p(1)}} \omega_{2}, \\
& 3\left\langle\iota_{\chi_{0}} \omega, j\right\rangle=\sum_{\ell=1}^{3} \iota_{I_{\ell} v_{\zeta_{\ell}}^{S p(1)} \omega_{2}}=\iota_{v_{\zeta_{2}}^{S p(1)}} g-\iota_{v_{\zeta_{1}}^{S p(1)}} \omega_{3}+\iota_{v_{\zeta_{3}}^{S p(1)}} \omega_{1}, \\
& 3\left\langle\iota_{\chi_{0}} \omega, k\right\rangle=\sum_{\ell=1}^{3} \iota_{I_{\ell} v_{\zeta_{\ell}}^{S p(1)} \omega_{3}}=\iota_{v_{\zeta_{3}}^{S p p(1)}} g+\iota_{v_{\zeta_{1}}^{S p(1)}} \omega_{2}-\iota_{v_{\zeta_{2}}^{S p(1)}} \omega_{1} .
\end{aligned}
$$

Therefore,

$$
3 \iota_{\chi_{0}} \omega=\iota_{\mathfrak{s p}(1)} g+4 \gamma
$$

Applying $d$ to the above formula and using $d \gamma=\omega$ and $\iota_{\chi_{0}} \omega=\mathcal{I} d \rho_{0}$, we obtain

$$
d \iota_{\mathfrak{s p}(1)} g=3 d \iota_{\chi_{0}} \omega-4 d \gamma=3 d \mathcal{I} d \rho_{0}-4 \omega .
$$

2.2.40 Corollary. If $M$ is a hyperkähler manifold with permuting $\operatorname{Spin}_{\varepsilon}^{G}(3)$-action, then

1. $\iota_{\chi_{0}} \iota_{\mathfrak{s p}(1)} g=-4 \iota_{\chi_{0}} \gamma$, i.e. $g\left(\chi_{0}, v_{\zeta}^{S p(1)}\right)=-4\left\langle\gamma\left(\chi_{0}\right), \zeta\right\rangle$,

2. $g\left(\chi_{0}, \mathcal{Y}\right)=-\frac{4}{3} \mu-\frac{1}{3} \iota_{\mathfrak{g}} \iota_{\mathfrak{s p}(1)} g$

3. $\left\|\chi_{0}\right\|^{2}=\frac{1}{3} \omega_{\zeta}\left(v_{\zeta}^{S p(1)}, \chi_{0}\right)-\frac{4}{3} \gamma\left(\mathcal{I}_{\zeta} \chi_{0}\right)$ for all $\zeta \in \mathfrak{s p}(1),\|\zeta\|^{2}=1$.

4. $\left\|v_{\zeta}^{S p(1)}\right\|^{2}=4\langle\rho, \zeta \otimes \zeta\rangle-3\left\langle d \rho\left(\chi_{0}\right), \zeta \otimes \zeta\right\rangle$ for all $\zeta \in \mathfrak{s p}(1)$.

Proof. All of these claims follow from inserting a vector field into the 1-form from Lemma 2.2.39:

1. $\iota_{\chi_{0}} \iota_{\mathfrak{s p}(1)} g=-4 \iota_{\chi_{0}} \gamma+3 \omega\left(\chi_{0}, \chi_{0}\right)=-4 \iota_{\chi_{0}} \gamma$.

2. $\iota_{\mathfrak{g}} \iota_{\mathfrak{s p}(1)} g=-4 \mu-3 \iota_{\chi_{0}} \omega=-4 \mu-3 g\left(\chi_{0}, \mathcal{Y}\right)$.

3. $\left\|\chi_{0}\right\|^{2}=-\iota_{\mathcal{I}_{\zeta} \chi_{0}} \iota_{\chi_{0}} \omega_{\zeta}=-\frac{1}{3} g\left(v_{\zeta}^{S p(1)}, \mathcal{I}_{\zeta} \chi_{0}\right)-\frac{4}{3}\langle\gamma, \zeta\rangle\left(\mathcal{I}_{\zeta} \chi_{0}\right)$.

$$
=-\frac{1}{3} \omega_{\zeta}\left(v_{\zeta}^{S p(1)}, \chi_{0}\right)-\frac{4}{3}\langle\gamma, \zeta\rangle\left(\mathcal{I}_{\zeta} \chi_{0}\right)
$$

4. $\left\|v_{\zeta}^{S p(1)}\right\|^{2}=-4\langle\gamma, \zeta\rangle\left(v_{\zeta}^{S p(1)}\right)+3 \omega_{\zeta}\left(\chi_{0}, v_{\zeta}^{S p(1)}\right)$ for all $\zeta \in \mathfrak{s p}(1)$. Finally, since $-\langle\gamma, \zeta\rangle\left(v_{\zeta}^{S p(1)}\right)=\langle\rho, \zeta \otimes \zeta\rangle$ and

$$
-\omega_{\zeta}\left(\chi_{0}, v_{\zeta}^{S p(1)}\right)=\left\langle\iota_{\chi_{0}} \pi_{S^{2} \mathfrak{s p}(1) \vee} \iota_{\mathfrak{s p}(1)} \omega, \zeta \otimes \zeta\right\rangle=\iota_{\chi_{0}} d\langle\rho, \zeta \otimes \zeta\rangle=\left\langle d \rho\left(\chi_{0}\right), \zeta \otimes \zeta\right\rangle
$$

the claim follows. 
2.2.41 Lemma $\left(\rho_{0}\right.$ and $\left.\left\|\chi_{0}\right\|^{2}\right)$. Let $M$ be a hyperkähler manifold with permuting Sp(1)-action. Then

$$
\left\|\chi_{0}\right\|^{2}=\frac{1}{9} \sum_{\ell=1}^{3}\left\|v_{\zeta_{\ell}}^{S p(1)}\right\|^{2}+\frac{4}{3} \rho_{0}
$$

In particular, $\rho_{0} \leq \frac{3}{4}\left\|\chi_{0}\right\|^{2}$.

Proof. Take a trace of the last equation in Corollary 2.2.40 to obtain

$$
\sum_{\ell=1}^{3}\left\|v_{\zeta_{\ell}}^{G}\right\|^{2}=-12 \rho_{0}+9 d \rho_{0}\left(\chi_{0}\right)=-12 \rho_{0}+9 \mathcal{L}_{\chi_{0}} \rho_{0}
$$

Since $\chi_{0}=\operatorname{grad}\left(\rho_{0}\right)$, we have $\left\|\chi_{0}\right\|^{2}=\mathcal{L}_{\chi_{0}} \rho_{0}$ and, hence, the claim follows.

\subsubsection{Manifolds with hyperkähler potential}

Among the hyperkähler manifolds with permuting $\operatorname{Spin}_{\varepsilon}^{G}(3)$-action, there are those hyperkähler manifolds with permuting action, which admit a hyperkähler potential.

2.2.42 Example (Swann bundles). Let $N$ be a compact quaternionic Kähler manifold with positive scalar curvature. Swann constructed [Swa91] a fibre bundle $M=\mathcal{U}(N) \rightarrow N$, with fibre $\mathbb{H}^{\times} / \pm 1$. The total space $\mathcal{U}(N)$ is hyperkähler and admits a permuting $S p(1)$ action with $\rho_{2} \equiv 0$ and hyperkähler potential $\rho_{0}=\frac{1}{2}\|\cdot\|^{2}$, where $\|\cdot\|$ is the norm on the fibres. Conversely, Swann proved that a hyperkähler manifold with permuting action and $\rho_{2} \equiv 0$ is locally homothetic to a Swann bundle ([Swa91, Thm. 5.9]). Examples for compact quaternionic Kähler manifolds with positive scalar curvature are Wolf spaces. These are the compact homogeneous quaternionic Kähler manifolds, i.e. $\mathbb{H P}^{n}=\frac{S p(n)}{S p(n-1) \times S p(1)}$, $G r_{2}\left(\mathbb{C}^{n}\right)=\frac{S U(n)}{S(U(n-2) \times U(2))}, \widetilde{G r}_{4}\left(\mathbb{R}^{n}\right)=\frac{S O(n)}{S O(n-4) \times S O(4)}$ and five quotients of the exotic simply connected compact Lie groups $G_{2}, F_{3}, E_{6}, E_{7}, E_{8}$. The corresponding hyperkähler manifold $M=\mathcal{U}(N)$ for a Wolf space $N$ is the minimal nilpotent coadjoint orbit of the simple complex Lie group (for details cf. [Swa91]).

\section{Swann's characterization of hyperkähler potentials}

Swann proves that $f \in C^{\infty}(M, \mathbb{R})$ is a hyperkähler potential if and only if $\nabla(d f)=g$ :

2.2.43 Proposition ([Swa91, Prop 5.5, Prop 5.6]).

Let $M$ be a hyperkähler manifold with $\omega \in \mathfrak{s p}(1)^{\vee} \otimes \Omega^{2}(M)$ and $f \in C^{\infty}(M, \mathbb{R})$. Then

$$
\nabla(d f)=g \quad \Leftrightarrow \quad d \mathcal{I} d f=2 \omega
$$

Furthermore, such a hyperkähler potential $f$ exists if and only if there is a local permuting $S p(1)$-action with $\chi_{2}=0$. 


\section{Equivalent characterizations of hyperkähler manifolds with permuting} action and potential $\rho_{0}$

We will provide a number of equivalent conditions for $\rho_{2}=0$. That the sixth, seventh and nineth condition are consequences of $\rho_{2}=0$, was observed in [BGM93]. The twelveth characterization first appeared in [Swa91]. We would also like to thank Henrik Schumacher for pointing out the eleventh characterization ([Sch10, Lem. 3.2.5]).

\subsubsection{Proposition (Hyperkähler manifold with potential).}

Let $M$ be a hyperkähler manifold with permuting Sp(1)-action. Then the following conditions are equivalent:

1. $\rho_{2}=0$

2. $\chi_{2}=0$

3. $\mathcal{I}_{\zeta} v_{\zeta}^{S p(1)}=\|\zeta\|^{2} \chi_{0}$ for all $\zeta \in \mathfrak{s p}(1)$

4. $\iota_{\chi_{0}} \omega=\iota_{\mathfrak{s p}(1)} g$

5. $2 \gamma=\iota_{\chi_{0}} \omega$

6. $\mathcal{L}_{\chi_{0}} \omega=2 \omega$

7. $\mathcal{L}_{\chi_{0}} \gamma=2 \gamma$

8. $\mathcal{L}_{\chi_{0}} \rho_{0}=2 \rho_{0}$

9. $\rho_{0}=\frac{1}{2}\left\|\chi_{0}\right\|^{2}$

10. $\left\|\chi_{0}\right\|^{2}=\frac{1}{3} \sum_{\ell=1}^{3}\left\|v_{\zeta_{\ell}}^{S p(1)}\right\|^{2}$

11. $\nabla \chi_{0}=\mathrm{id}_{T M}$

12. $\nabla\left(d \rho_{0}\right)=g$

13. $\rho_{0}$ is a hyperkähler potential, i.e. $d \mathcal{I} d \rho_{0}=2 \omega$ 
Proof. The following diagram shows which implications we prove (including the proofs in Remark 2.2.45). Next to the arrows we provide a hint to what is used in the proof:

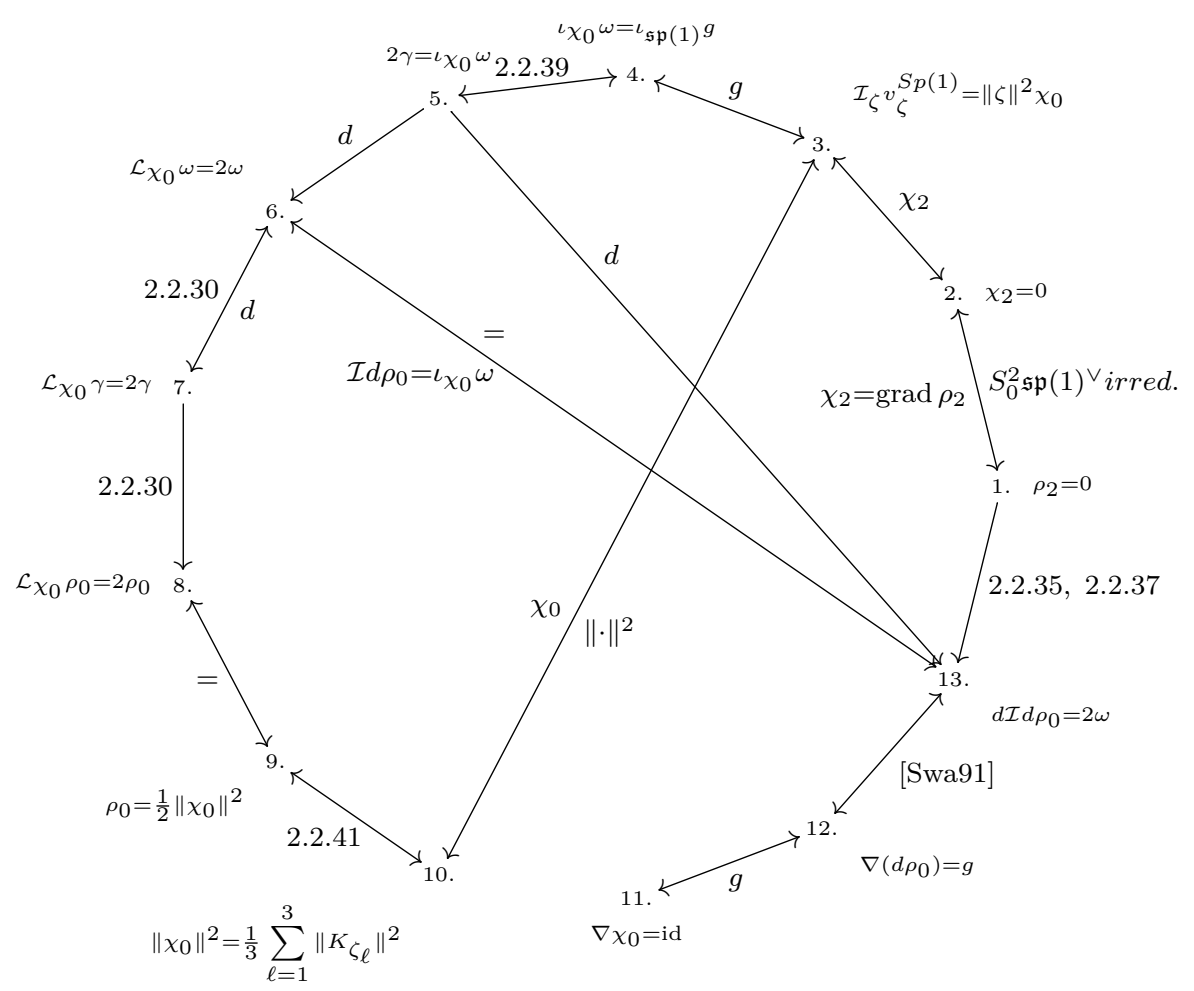

We first prove that the conditions $1,2,3$ are equivalent:

$1 \Leftrightarrow 2$ The condition $\rho_{2}=0$ implies $\chi_{2}=0$ since $\chi_{2}=\operatorname{grad}\left(\rho_{2}\right)$. On the other hand, if $0=\chi_{2}=\operatorname{grad}\left(\rho_{2}\right)$, then $\rho_{2}$ has to be locally constant. But $\rho_{2} \in C^{\infty}\left(M, S_{0}^{2} \mathfrak{s p}(1)\right)$ is equivariant and the $S p(1)$-representation $S_{0}^{2} \mathfrak{s p}(1)^{\vee}$ is irreducible. Now if for some $x \in M: \rho_{2}(x) \neq 0$, then there exists $g \in S p(1)$ such that $\rho_{2}(x) \neq g \rho_{2}(x)$. Since $S p(1)$ is compact and connected, the exponential map is surjective and we can write $g=\exp (t \xi)$ for some $\xi \in \mathfrak{s p}(1)$. Since $x$ and $g x$ are in the same component of $M$ (connected by the path $\exp (t \xi) x$ ), we know that $\rho_{2}(g x)=\rho_{2}(x)$. However, this leads to the contraction $\rho_{2}(x) \neq g \rho_{2}(x)=\rho_{2}(g x)=\rho_{2}(x)$. Hence, we can conclude that $\rho_{2}=0$.

$1 \Rightarrow 13$ We proved this in Lemma 2.2.35 and Corollary 2.2.37.

$2 \Leftrightarrow 3$ For $\zeta \in \mathfrak{s p}(1),\|\zeta\|^{2}=1$, we have

$$
\left\langle\chi_{2}, \zeta \otimes \zeta\right\rangle=\left\langle\chi_{2}, \zeta \otimes \zeta\right\rangle=\chi_{0}-\mathcal{I}_{\zeta} v_{\zeta}^{S p(1)}
$$

Hence, $\chi_{2}=0$ if and only if $\chi_{0}=\mathcal{I}_{\zeta} v_{\zeta}^{S p(1)}$ for all $\zeta \in \mathfrak{s p}(1),\|\zeta\|^{2}=1$.

$3 \Rightarrow 4$ For $\zeta \in \mathfrak{s p}(1)$

$$
\omega_{\zeta}\left(\chi_{0}, v\right)=-g\left(\mathcal{I}_{\zeta} \chi_{0}, v\right)=g\left(v_{\zeta}^{S p(1)}, v\right),
$$

and therefore $\iota_{\chi_{0}} \omega=\iota_{\mathfrak{s p}(1)} g$. 
$4 \Rightarrow 5$ We know from Lemma 2.2.39 that $\iota_{\mathfrak{s p}(1)} g=3 \iota_{\chi_{0}} \omega-4 \gamma$, and therefore,

$$
\iota_{\mathfrak{s p}(1)} g=\iota_{\chi_{0}} \omega=\frac{1}{3} \iota_{\mathfrak{s p}(1)} g+\frac{4}{3} \gamma .
$$

Thus, $\frac{2}{3} \iota_{\mathfrak{s p}(1)} g=\frac{4}{3} \gamma$ and therefore, $2 \gamma=\iota_{\mathfrak{s p}(1)} g=\iota_{\chi_{0}} \omega$.

$5 \Rightarrow 6 \mathcal{L}_{\chi_{0}} \omega=d \iota_{\chi_{0}} \omega+\iota_{\chi_{0}} d \omega=d \iota_{\chi_{0}} \omega=2 d \gamma=2 \omega$.

$6 \Rightarrow 7$ We use Note 2.2.30 to compute

$$
\begin{aligned}
\mathcal{L}_{\chi_{0}} \gamma & =\frac{1}{2} \mathcal{L}_{\chi_{0}}\left(\pi_{\mathfrak{s p}(1) \vee} \iota_{\mathfrak{s p}(1)} \omega\right)=\frac{1}{2} \pi_{\mathfrak{s p}(1) \vee} \mathcal{L}_{\chi_{0}} \iota_{\mathfrak{s p}(1)} \omega \\
& =\frac{1}{2} \pi_{\mathfrak{s p}(1)^{\vee} \iota_{\mathfrak{s p}(1)}} \mathcal{L}_{\chi_{0}} \omega=\frac{1}{2} \pi_{\mathfrak{s p}(1) \vee \iota_{\mathfrak{s p}(1)}} 2 \omega \\
& =2 \gamma
\end{aligned}
$$

$7 \Rightarrow 8$ From $\mathcal{L}_{\chi_{0}} \gamma=2 \gamma$ and $\mathcal{L}_{\chi_{0}} \iota_{\mathfrak{s p}(1)}=\iota_{\mathfrak{s p}(1)} \mathcal{L}_{\chi_{0}}$, we obtain

$$
\mathcal{L}_{\chi_{0}} \rho_{0}=\frac{1}{3} \mathcal{L}_{\chi_{0}} \operatorname{tr}\left(\iota_{\mathfrak{s p}(1)} \gamma\right)=\frac{1}{3} \operatorname{tr}\left(\iota_{\mathfrak{s p}(1)} \mathcal{L}_{\chi_{0}} \gamma\right)=\frac{2}{3} \operatorname{tr}\left(\iota_{\mathfrak{s p}(1)} \gamma\right)=2 \rho_{0}
$$

$8 \Rightarrow 9$ Using $\mathcal{L}_{\chi_{0}} \rho_{0}=2 \rho_{0}$, we obtain $g\left(\chi_{0}, \chi_{0}\right)=d \rho_{0}\left(\chi_{0}\right)=\mathcal{L}_{\chi_{0}} \rho_{0}=2 \rho_{0}$.

$9 \Rightarrow 10$ We use Lemma 2.2.41 to compute

$$
2 g\left(\chi_{0}, \chi_{0}\right)=4 \rho_{0}=3 g\left(\chi_{0}, \chi_{0}\right)-\frac{1}{3} \sum_{\ell=1}^{3} g\left(v_{\zeta_{\ell}}^{S p(1)}, v_{\zeta_{\ell}}^{S p(1)}\right),
$$

and therefore, $g\left(\chi_{0}, \chi_{0}\right)=\frac{1}{3} \sum_{\ell=1}^{3} g\left(v_{\zeta_{\ell}}^{S p(1)}, v_{\zeta_{\ell}}^{S p(1)}\right)$.

$10 \Rightarrow 3$ Let $w=\frac{1}{3} \sum_{\ell=1}^{3} v_{\ell}$ and assume that $\|w\|^{2}=\frac{1}{3} \sum_{\ell=1}^{3}\left\|v_{\ell}\right\|^{2}$. Then

$$
\frac{1}{3} \sum_{\ell=1}^{3}\left\|v_{\ell}\right\|^{2}=\|w\|^{2}=\frac{1}{9} \sum_{\ell=1}^{3}\left\|v_{\ell}\right\|^{2}+\frac{2}{9} \sum_{1 \leq m<n \leq 3}\left\langle v_{m}, v_{n}\right\rangle,
$$

and therefore, $\sum_{\ell=1}^{3}\left\|v_{\ell}\right\|^{2}=\sum_{1 \leq m<n \leq 3}\left\langle v_{m}, v_{n}\right\rangle$. We conclude that

$$
0=\sum_{\ell=1}^{3}\left\|v_{\ell}\right\|^{2}-\sum_{1 \leq m<n \leq 3}\left\langle v_{m}, v_{n}\right\rangle=\frac{1}{2} \sum_{1 \leq m<n \leq 3}\left\|v_{m}-v_{n}\right\|^{2},
$$

and hence, $v_{1}=v_{2}=v_{3}=v$. We apply this to $v_{\ell}:=I_{\ell} v_{\zeta_{\ell}}^{S p(1)}, w=\chi_{0}$ to prove the assertion.

$5 \Rightarrow 13 d \mathcal{I} d \rho_{0}=d \iota_{\chi_{0}} \omega=2 d \gamma=2 \omega$.

$11 \Rightarrow 12$ For all vector fields $v, w \in \Gamma(M, T M)$ we have

$$
\begin{aligned}
\nabla_{v}\left(d \rho_{0}\right)(w) & =\nabla_{v}\left(d \rho_{0}(w)\right)-d \rho_{0}\left(\nabla_{v}(w)\right)=\nabla_{v}\left(g\left(\chi_{0}, w\right)\right)-g\left(\chi_{0}, \nabla_{v}(w)\right) \\
& =g\left(\nabla_{v} \chi_{0}, w\right)=g(v, w),
\end{aligned}
$$

and therefore $\nabla\left(d \rho_{0}\right)=g$. 
$12 \Rightarrow 11$ For all vector fields $v, w \in \Gamma(M, T M)$ we have

$$
\begin{aligned}
g\left(\nabla_{v} \chi_{0}, w\right) & =d\left(g\left(\chi_{0}, w\right)\right)(v)-g\left(\chi_{0}, \nabla_{v} w\right)=d\left(d \rho_{0}(w)\right)(v)-d \rho_{0}\left(\nabla_{v} w\right) \\
& =\nabla_{v}\left(d \rho_{0}\right)(w)=g(v, w),
\end{aligned}
$$

and therefore $\nabla\left(\chi_{0}\right)=\mathrm{id}_{T M}$.

$12 \Leftrightarrow 13$ Swann proves in [Swa91, Prop. 5.6] that $f \in C^{\infty}(M, \mathbb{R})$ is a hyperkähler potential if and only if $\nabla(d f)=g$. Therefore, $\nabla\left(d \rho_{0}\right)=g$.

$13 \Rightarrow 6$ We always have $\iota_{\chi 0} \omega=I d \rho_{0}$. Therefore, if $\rho_{0}$ is a hyperkähler potential, i.e. $d I d \rho_{0}=2 \omega$, we obtain

$$
\mathcal{L}_{\chi_{0}} \omega=\iota_{\chi_{0}} d \omega+d \iota_{\chi_{0}} \omega=d \iota_{\chi_{0}} \omega=d \mathcal{I} d \rho_{0}=2 \omega .
$$

2.2.45 Remark. We will now prove some more implications directly:

$$
\begin{aligned}
& 4 \Rightarrow 3 g\left(\mathcal{I}_{\zeta} v_{\zeta}^{S p(1)}, v\right)=-g\left(v_{\zeta}^{S p(1)}, \mathcal{I}_{\zeta} v\right)=-\omega_{\zeta}\left(\chi_{0}, \mathcal{I}_{\zeta} v\right)=\|\zeta\|^{2} g\left(\chi_{0}, v\right) \text { for all } v \in T M \text { and } \\
& \zeta \in \mathfrak{s p}(1) \text {. } \\
& 5 \Rightarrow 4 \text { Using } \iota_{\mathfrak{s p}(1)} g=-4 \gamma+3 \iota_{\chi_{0}} \omega \text { from Lemma 2.2.39, we obtain } \iota_{\mathfrak{s p}(1)} g=-4 \gamma+3 \iota_{\chi_{0}} \omega= \\
& -2 \iota_{\chi_{0}} \omega+3 \iota_{\chi_{0}} \omega=\iota_{\chi_{0}} \omega \text {. } \\
& 7 \Rightarrow 6 \mathcal{L}_{\chi_{0}} \omega=\mathcal{L}_{\chi_{0}} d \gamma=d \mathcal{L}_{\chi_{0}} \gamma=2 d \gamma=2 \omega \\
& 6 \Leftrightarrow 13 \text { Note that } \mathcal{L}_{\chi_{0}} \omega=d \iota_{\chi_{0}} \omega=d \mathcal{I} d \rho_{0} \text {. }
\end{aligned}
$$

2.2.46 Corollary ([Cal10, Prop. 3.2.6], [Sch10, Lem. 3.4.1]). If one of the conditions in the previous proposition holds, then

1. $d \mu\left(\chi_{0}\right)=\mathcal{L}_{\chi_{0}} \mu=2 \mu$

2. $\mu=\frac{1}{2} \iota_{\mathfrak{g}} \iota_{\chi_{0}} \omega$, or equivalently, $\mu=\frac{1}{2} \iota_{\mathfrak{s p}(1)} \iota_{\mathfrak{g}} g$.

Proof.

1. $d \mu\left(\chi_{0}\right)=\mathcal{L}_{\chi_{0}} \mu=\mathcal{L}_{\chi_{0}} \iota_{\mathfrak{g}} \gamma=\iota_{\mathfrak{g}} \mathcal{L}_{\chi_{0}} \gamma=2 \iota_{\mathfrak{g}} \gamma=2 \mu$

2. We use $2 \gamma=\iota_{\chi_{0}} \omega$ to obtain $\mu=\iota_{\mathfrak{g}} \gamma=\frac{1}{2} \iota_{\mathfrak{g}} \iota_{\chi_{0}} \omega$.

2.2.47 Remark. Another way to obtain $\mu=\frac{1}{2} \iota_{\mathfrak{g}} \iota_{\chi_{0}} \omega$ is to observe that $\chi_{2}=0$ implies $\chi_{A l t}=v^{S p(1)}$ and $\mathcal{I}_{\zeta} v_{\zeta}^{S p(1)}=\|\zeta\|^{2} \chi_{0}$. Combining this with $\mu=\frac{1}{2} \iota_{\mathfrak{g}} \iota_{\chi_{A l t}}$ g, this gives

$$
\mu=\frac{1}{2} \iota_{\mathfrak{g}} \iota_{\chi_{A l t}} g=\frac{1}{2} \iota_{\mathfrak{g}} \iota_{\mathfrak{s p}(1)} g=\frac{1}{2} \iota_{\mathfrak{g}} \iota_{\chi_{0}} \omega .
$$


2.2.48 Remark. As we have seen in Example 2.2.15, even if $\rho_{2} \not \equiv 0$ a hyperkähler potential can still exist.

Also note that if a vector field $\hat{\chi} \in \Gamma(M, T M)$ satisfies $\nabla \hat{\chi}=\operatorname{id}_{T M}$, then $f:=\frac{1}{2}\|\hat{\chi}\|^{2} \in$ $C^{\infty}(M, \mathbb{R})$ satisfies

$$
d f=\frac{1}{2} d g(\hat{\chi}, \hat{\chi})=g(\nabla \hat{\chi}, \hat{\chi})=g(\cdot, \hat{\chi}),
$$

and therefore, $\hat{\chi}=\operatorname{grad}(f)$. In particular, $\nabla \operatorname{grad}(f)=\mathrm{id}_{T M}$, which using the same proof as in " $11 \Rightarrow 12$ " of Proposition 2.2.44 implies $\nabla(d f)=g$. Therefore, by Swann's criterion (Proposition 2.2.43), $f=\frac{1}{2}\|\hat{\chi}\|^{2}$ is a hyperkähler potential.

2.2.49 Remark (Uniqueness of hyperkähler potentials). Let $M$ be a connected hyperkähler manifold of dimension $4 n$.

If $f$ is a hyperkähler potential and $c \in \mathbb{R}$, then $f+c$ is also a hyperkähler potential. However, the previous remark provides a natural normalization, i.e. $\frac{1}{2}\|\operatorname{grad}(f)\|^{2}$. This was also observed in [Sch10, Rem. 3.2.6].

If $M$ has two hyperkähler potentials $f_{1}$ and $f_{2}$ with $d f_{1} \neq d f_{2}$, then $\nabla\left(\operatorname{grad}\left(f_{1}-f_{2}\right)\right)=0$. Hence, $v:=\operatorname{grad}\left(f_{1}-f_{2}\right)$ is a parallel, nowhere vanishing vector field. Furthermore, $I_{1} v, I_{2} v$ and $I_{3} v$ are also parallel and the holonomy group reduces to $S p(n-1) \subset S p(n)$ and $M$ is locally isometric to a product of a $4 n-4$-dimensional hyperkähler manifold and $\mathrm{H}$. If $M$ is simply-connected and complete, then it is globally a product of this form.

In particular, if $M$ is irreducible, then the hyperkähler potential is unique up to a constant.

Conversely, if $v \in \Gamma(M, T M)$ is a parallel vector field and $f=\frac{1}{2}\|\operatorname{grad}(f)\|^{2}$ is a (normalized) hyperkähler potential, then $\frac{1}{2}\|\operatorname{grad}(f)+v\|^{2}=f+d f(v)+\frac{1}{2}\|v\|^{2}$ is a hyperkähler potential since $\nabla(\operatorname{grad}(f)+v)=\operatorname{id}_{T M}$. Therefore, if $f$ is a hyperkähler potential on $M$, then every other hyperkähler potential on $M$ is of the form $f+d f(v)+c$ for some parallel vector field $v$ and a constant $c$. If $M$ admits a hyperkähler potential, then the dimension of the space of hyperkähler potentials is the sum of the dimension of the space of parallel vector fields and the number of connected components of $M$.

\subsubsection{Example (hyperkähler potential on quaternionic vector spaces).}

Consider $M=\mathbb{H}^{n}$ from Example 2.2.6 with the action of $S p(1)$ on $\mathbb{H}^{n}$ given by left multiplication in each component. The fundamental vector field for this action is

$$
\left(v_{\zeta}^{S p(1)}\right)_{x}=\left.\frac{d}{d t} \exp (-t \zeta) x\right|_{t=0}=-\zeta x \in \mathbb{H}^{n}=T_{x} \mathbb{H}^{n} \text { for all } x \in \mathbb{H}^{n}, \zeta \in \mathfrak{s p}(1) .
$$

We obtain

$$
\mathcal{I}_{\zeta}\left(v_{\zeta}^{S p(1)}\right)_{x}=-\zeta \zeta x=x \in \mathbb{H}^{n}=T_{x} \mathbb{H}^{n} \text { for all } \zeta \in \mathfrak{s p}(1),\|\zeta\|^{2}=1 .
$$

The vector field $\chi_{0}=\mathcal{I}_{\zeta} v_{\zeta}^{S p(1)}$ is independent of $\zeta \in \mathfrak{s p}(1),\|\zeta\|^{2}=1$. This is the Euler vector field $\left.\chi_{0}\right|_{x}=x \in \mathbb{H}^{n}=T_{x} \mathbb{H}^{n}$. The hyperkähler potential is

$$
\rho_{0}(x)=\frac{1}{2} g^{M}\left(\left.\chi_{0}\right|_{x},\left.\chi_{0}\right|_{x}\right)=\frac{1}{2}\left\|\left.\chi_{0}\right|_{x}\right\|^{2}=\frac{1}{2}\|x\|^{2} .
$$


It follows from Remark 2.2.49 that an arbitrary hyperkähler potential is of the form $f(x)=\frac{1}{2}\|x\|^{2}+\operatorname{Re}\left(x^{*} v\right)+c$ for some $v \in \mathbb{H}^{n}$ and $c \in \mathbb{R}$. The corresponding permuting $S p(1)$-action from Proposition 2.2.43 is obtained from the above action and the translation by $v$.

\subsection{8 $\operatorname{Spin}_{\varepsilon}^{G}(3)^{\star}$-module generated by $\omega$}

In this section, we show how the forms $\gamma, \rho, \rho_{0}, \rho_{2}$ naturally appear from the permuting $\operatorname{Spin}_{\varepsilon}^{G}(3)$-action on $M$. Recall the following definition ([GS99]):

2.2.51 Definition. Given a smooth action $G \curvearrowright M$, we have an action of the $\mathbb{Z}$-graded Lie superalgebra $\tilde{\mathfrak{g}}:=\mathfrak{g}[-1] \oplus \mathfrak{g} \oplus \mathbb{R}[1]$ on $\Omega^{*}(M)$ by derivations:

$$
\mathfrak{g}[-1] \text { acts as the insertion operator } \iota_{\mathfrak{g}},
$$

$\mathfrak{g}$ acts as the Lie derivative $\mathcal{L}_{\mathfrak{g}}$,

$1 \in \mathbb{R}[1]$ acts as the exterior derivative $d$.

Here, $\mathfrak{g}[-1]$ is the Lie algebra of $G$ sitting in degree $-1, \mathfrak{g}$ is in degree 0 and $\mathbb{R}[1]$ is in degree 1 . The Lie bracket on $\tilde{\mathfrak{g}}$ is defined in such a way that the usual commutation relations between Lie derivative, insertion operation and exterior derivative hold in $\tilde{\mathfrak{g}}$.

A $G^{\star}$-module is a $\mathbb{Z}$-graded vector space $A$ with a linear $G$-action and a $G$-equivariant $\tilde{\mathfrak{g}}$-action such that the infinitesimal $G$-action on $A$ coincides with the action of $\mathfrak{g} \subset \tilde{\mathfrak{g}}$.

A morphism of $G^{\star}$-modules is a degree-preserving linear map which commutes with the $G$-action and the $\tilde{\mathfrak{g}}$-action.

Given a hyperkähler manifold $M$ with a permuting $\operatorname{Spin}_{\varepsilon}^{G}(3)$-action, we can understand $\Omega^{*}(M)$ as a $\operatorname{Spin}_{\varepsilon}^{G}(3)^{\star}$-module and study the $\operatorname{Spin}_{\varepsilon}^{G}(3)^{\star}$-submodule generated by one of the symplectic forms $\omega_{1}$.

Since the Lie derivative $\mathcal{L}_{\mathfrak{s p i n}_{\varepsilon}^{G}(3)}$ generates the 3 -dimensional space of 2 -forms spanned by $\omega_{1}, \omega_{2}, \omega_{3}$, but leaves $\omega \in \mathfrak{s p}(1)^{\vee} \otimes \Omega^{2}(M)$ invariant, we can equivalently iterate the insertion operation:

$$
\sum_{\ell=0}^{2}\left(\iota_{\mathfrak{s p i n} G}{ }_{\varepsilon}(3)\right)^{\ell} \omega \in \bigoplus_{\ell=0}^{2}\left(\bigwedge^{\ell} \mathfrak{s p i n}_{\varepsilon}^{G}(3)^{\vee} \otimes \mathfrak{s p}(1)^{\vee} \otimes \Omega^{2-\ell}(M)\right)^{\operatorname{Spin}_{\varepsilon}^{G}(3)}
$$

Since everything generated by these insertion operations is $\operatorname{Spin}_{\varepsilon}^{G}(3)$-invariant, the Lie derivatives do not produce any new elements of the $\operatorname{Spin}_{\varepsilon}^{G}(3)^{\star}$-module. The same holds for the exterior derivative $d$, since $d \omega=0, d \mathcal{L}_{\mathfrak{s p i n}_{\varepsilon}^{G}(3)}=\mathcal{L}_{\mathfrak{s p i n}_{\varepsilon}^{G}(3)} d$ and $d \iota_{\mathfrak{s p i n}_{\varepsilon}^{G}(3)}=$ $\mathcal{L}_{\mathfrak{s p i n}_{\varepsilon}^{G}(3)}-\iota_{\mathfrak{s p i n}_{\varepsilon}^{G}(3)} d$. Therefore, the image of $\bigoplus_{\ell=0}^{2}\left(\Lambda^{\ell} \mathfrak{s p i n}_{\varepsilon}^{G}(3)^{\vee} \otimes \mathfrak{s p}(1)^{\vee}\right)$ in $\Omega^{*}(M)$ is the $\operatorname{Spin}_{\varepsilon}^{G}(3)^{\star}$-submodule of $\Omega^{*}(M)$ generated by $\omega_{1}$. This also contains all the differential forms from Proposition 2.2.7. To see this, we use the following decomposition of 
$\operatorname{Spin}_{\varepsilon}^{G}(3)$-representations:

$$
\begin{aligned}
\bigwedge^{0}\left(\mathfrak{s p}(1)^{\vee} \oplus \mathfrak{g}^{\vee}\right) \otimes \mathfrak{s p}(1)^{\vee} & \cong \mathfrak{s p}(1)^{\vee} \\
\bigwedge^{1}\left(\mathfrak{s p}(1)^{\vee} \oplus \mathfrak{g}^{\vee}\right) \otimes \mathfrak{s p}(1)^{\vee} & \cong \mathfrak{s p}(1)^{\vee} \oplus S^{2} \mathfrak{s p}(1)^{\vee} \oplus \mathfrak{g}^{\vee} \otimes \mathfrak{s p}(1)^{\vee} \\
& \cong \mathfrak{s p}(1)^{\vee} \oplus S_{0}^{2} \mathfrak{s p}(1)^{\vee} \oplus \mathbb{R} \oplus \mathfrak{g} \otimes \mathfrak{s}(1)^{\vee} \\
\bigwedge^{2}\left(\mathfrak{s p}(1)^{\vee} \oplus \mathfrak{g}^{\vee}\right) \otimes \mathfrak{s p}(1)^{\vee} & \cong\left(\bigwedge^{2} \mathfrak{s p}(1)^{\vee} \oplus \mathfrak{s p}(1)^{\vee} \otimes \mathfrak{g}^{\vee} \oplus \bigwedge^{2} \mathfrak{g}^{\vee}\right) \otimes \mathfrak{s p}(1)^{\vee} \\
& \cong\left(\mathfrak{s p}(1)^{\vee} \oplus \mathfrak{s p}(1)^{\vee} \otimes \mathfrak{g}^{\vee} \oplus \bigwedge^{2} \mathfrak{g}^{\vee}\right) \otimes \mathfrak{s p}(1)^{\vee} \\
& \cong\left(\mathbb{R} \oplus \mathfrak{g}^{\vee}\right) \otimes\left(\mathfrak{s p}(1)^{\vee} \oplus S_{0}^{2} \mathfrak{s p}(1)^{\vee} \oplus \mathbb{R}\right) \oplus \bigwedge^{2} \mathfrak{g}^{\vee} \otimes \mathfrak{s p}(1)^{\vee}
\end{aligned}
$$

The representations and the corresponding components of $\sum_{\ell=0}^{2}\left(\iota_{\mathfrak{s p i n}_{\varepsilon}^{G}(3)}\right)^{\ell} \omega$ are listed in the following table:

\begin{tabular}{|c|c|}
\hline representation & component \\
\hline \hline $\mathfrak{s p}(1)^{\vee} \otimes \Omega^{2}(M)$ & $\omega$ \\
$\mathfrak{s p}(1)^{\vee} \otimes \Omega^{1}(M)$ & $\gamma=\frac{1}{2} \pi_{\mathfrak{s p}(1) \vee} \iota_{\mathfrak{s p}(1)} \omega$ \\
$S^{2} \mathfrak{s p}(1)^{\vee} \otimes \Omega^{1}(M)$ & $d \rho=\pi_{S^{2} \mathfrak{s p}(1)^{\vee} \iota_{\mathfrak{s p}(1)} \omega}$ \\
$S_{0}^{2} \mathfrak{s p}(1)^{\vee} \otimes \Omega^{1}(M)$ & $d \rho_{2}=\pi_{S_{0}^{2} \mathfrak{s p}(1)^{\vee} \iota_{\mathfrak{s p}(1)} \omega}$ \\
$\Omega^{1}(M)$ & $d \rho_{0}=-\frac{1}{3} \operatorname{tr}\left(\iota_{\mathfrak{s p}(1)} \omega\right)$ \\
$\mathfrak{g}^{\vee} \otimes \mathfrak{s p}(1)^{\vee} \otimes \Omega^{1}(M)$ & $-d \mu=\iota_{\mathfrak{g}} \omega$ \\
$\mathfrak{g}^{\vee} \otimes \mathfrak{s p}(1)^{\vee} \otimes \Omega^{0}(M)$ & $\mu=\iota_{\mathfrak{g}} \gamma$ \\
$\mathfrak{s p}(1)^{\vee} \otimes \mathfrak{s p}(1)^{\vee} \otimes \Omega^{0}(M)$ & $\rho=-\iota_{\mathfrak{s p}(1)}$ \\
$\mathfrak{s p}(1)^{\vee} \otimes \Omega^{0}(M)$ & $\pi_{\wedge^{2} \mathfrak{s p}(1)^{\vee} \rho=0} \rho_{2}$ \\
$S_{0}^{2} \mathfrak{s p}(1)^{\vee} \otimes \Omega^{0}(M)$ & $\rho_{0}$ \\
$\Omega^{0}(M)$ & $\rho_{\mathfrak{g}} d \rho=\mathcal{L}_{\mathfrak{g}} \rho=0$ \\
$\mathfrak{g}^{\vee} \otimes S^{2} \mathfrak{s p}(1)^{\vee} \otimes \Omega^{0}(M)$ & $\iota_{\mathfrak{g}} d \rho_{2}=\mathcal{L}_{\mathfrak{g}} \rho_{2}=0$ \\
$\mathfrak{g}^{\vee} \otimes S_{0}^{2} \mathfrak{s p}(1)^{\vee} \otimes \Omega^{0}(M)$ & $\iota_{\mathfrak{g}} d \rho_{0}=\mathcal{L}_{\mathfrak{g}} \rho_{0}=0$ \\
$\mathfrak{g}^{\vee} \otimes \Omega^{0}(M)$ & $\iota_{\mathfrak{g}}^{2} \omega=\iota_{\mathfrak{g}} d \mu=-\delta_{\mathfrak{g}} \mu$ \\
$\wedge^{2} \mathfrak{g}^{\vee} \otimes \mathfrak{s p}(1)^{\vee} \otimes \Omega^{0}(M)$
\end{tabular}

Note that for $\mathfrak{s p}(1)^{\vee} \otimes \Omega^{1}(M), \mathfrak{g}^{\vee} \otimes \mathfrak{s p}(1)^{\vee} \otimes \Omega^{0}(M), \mathfrak{s p}(1)^{\vee} \otimes \mathfrak{s p}(1)^{\vee} \otimes \Omega^{0}(M)$ and

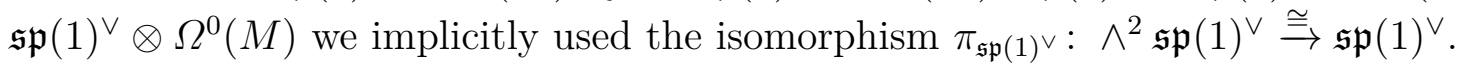

Furthermore, this explains why apart from $\omega$, the forms $\gamma, \rho=\left(\rho_{0}, \rho_{2}\right)$ and $\mu$ naturally appear on a hyperkähler manifold with permuting $\operatorname{Spin}_{\varepsilon}^{G}(3)$-action.

From this table, we also obtain an 18-dimensional, universal $S p(1)^{\star}$-module $A^{\text {perm }}$

$$
A^{\text {perm }}:=\mathfrak{s p}(1)[2] \oplus \mathfrak{s p}(1)[1] \oplus S_{0}^{2} \mathfrak{s p}(1)[1] \oplus \mathbb{R}[1] \oplus S_{0}^{2} \mathfrak{s p}(1) \oplus \mathbb{R}
$$


for permuting actions, with differential given by

$$
\begin{aligned}
\left.d\right|_{\mathfrak{s p}(1)[2]} & =0 \\
\left.d\right|_{\mathfrak{s p}(1)[1]} & =\mathrm{id}_{\mathfrak{s p}(1)}: \mathfrak{s p}(1)[1] \rightarrow \mathfrak{s p}(1)[2], \\
\left.d\right|_{S_{0}^{2} \mathfrak{s p}(1)[1]} & =0 \\
\left.d\right|_{\mathbb{R}[1]} & =0 \\
\left.d\right|_{S_{0}^{2} \mathfrak{s p}(1)} & =\mathrm{id}_{S_{0}^{2} \mathfrak{s p}(1)}: S_{0}^{2} \mathfrak{s p}(1) \rightarrow S_{0}^{2} \mathfrak{s p}(1)[1], \\
\left.d\right|_{\mathbb{R}} & =\mathrm{id}_{\mathfrak{s p}(1)}: \mathbb{R} \rightarrow \mathbb{R}[1],
\end{aligned}
$$

the Lie derivatives

$$
\begin{aligned}
\left.\mathcal{L}_{\mathfrak{s p}(1)}\right|_{\mathfrak{s p}(1)[2]} & =[\cdot, \cdot]: \mathfrak{s p}(1) \otimes \mathfrak{s p}(1)[2] \rightarrow \mathfrak{s p}(1)[2] \\
\left.\mathcal{L}_{\mathfrak{s p}(1)}\right|_{\mathfrak{s p}(1)[1]} & =[\cdot, \cdot]: \mathfrak{s p}(1) \otimes \mathfrak{s p}(1)[1] \rightarrow \mathfrak{s p}(1)[1] \\
\left.\mathcal{L}_{\mathfrak{s p}(1)}\right|_{S_{0}^{2} \mathfrak{s p}(1)[1]} & =\left([\cdot, \cdot] \otimes \mathrm{id}_{\mathfrak{s p}(1)}+\left(\mathrm{id}_{\mathfrak{s p}(1)} \otimes[\cdot, \cdot]\right) \circ \tau_{12}\right): \mathfrak{s p}(1) \otimes S_{0}^{2} \mathfrak{s p}(1)[1] \rightarrow S_{0}^{2} \mathfrak{s p}(1)[1] \\
\left.\mathcal{L}_{\mathfrak{s p}(1)}\right|_{\mathbb{R}[1]} & =0 \\
\left.\mathcal{L}_{\mathfrak{s p}(1)}\right|_{S_{0}^{2} \mathfrak{p p}(1)} & =\left([\cdot, \cdot] \otimes \mathrm{id}_{\mathfrak{s p}(1)}+\left(\mathrm{id}_{\mathfrak{s p}(1)} \otimes[\cdot, \cdot]\right) \circ \tau_{12}\right): \mathfrak{s p}(1) \otimes S_{0}^{2} \mathfrak{s p}(1) \rightarrow S_{0}^{2} \mathfrak{s p}(1) \\
\left.\mathcal{L}_{\mathfrak{s p}(1)}\right|_{\mathbb{R}} & =0
\end{aligned}
$$

where $\tau_{12}: \mathfrak{s p}(1) \otimes \mathfrak{s p}(1) \otimes \mathfrak{s p}(1) \rightarrow \mathfrak{s p}(1) \otimes \mathfrak{s p}(1) \otimes \mathfrak{s p}(1)$ is $\zeta \otimes \zeta^{\prime} \otimes \zeta^{\prime \prime} \mapsto \zeta^{\prime} \otimes \zeta \otimes \zeta^{\prime \prime}$ Finally, the insertion operations are

$$
\begin{aligned}
\left.\iota_{\mathfrak{s p}(1)}\right|_{\mathfrak{s p}(1)[2]} & =\left([\cdot, \cdot], \operatorname{pr}_{S_{0}^{2} \mathfrak{s p}(1)}, \frac{1}{3} \operatorname{tr}\right): \mathfrak{s p}(1)[-1] \otimes \mathfrak{s p}(1)[2] \rightarrow \mathfrak{s p}(1)[1] \oplus S_{0}^{2} \mathfrak{s p}(1)[1] \oplus \mathbb{R}[1], \\
\left.\iota_{\mathfrak{s p}(1)}\right|_{\mathfrak{s p}(1)[1]} & =\left(-\operatorname{pr}_{S_{0}^{2} \mathfrak{s p}(1)},-\frac{1}{3} \operatorname{tr}\right): \mathfrak{s p}(1)[-1] \otimes \mathfrak{s p}(1)[1] \rightarrow S_{0}^{2} \mathfrak{s p}(1) \oplus \mathbb{R}, \\
\left.\iota_{\mathfrak{s p}(1)}\right|_{S_{0}^{2} \mathfrak{s p}(1)[1]} & =\left([\cdot, \cdot] \otimes \mathrm{id}_{\mathfrak{s p}(1)}+\left(\mathrm{id}_{\mathfrak{s p}(1)} \otimes[\cdot, \cdot]\right) \circ \tau_{12}\right): \mathfrak{s p}(1)[-1] \otimes S_{0}^{2} \mathfrak{s p}(1)[1] \rightarrow S_{0}^{2} \mathfrak{s p}(1) \\
\left.\iota_{\mathfrak{s p}(1)}\right|_{\mathbb{R}[1]} & =0 \\
\left.\iota_{\mathfrak{s p}(1)}\right|_{S_{0}^{2} \mathfrak{s p}(1)} & =0 \\
\left.\iota_{\mathfrak{s p}(1)}\right|_{\mathbb{R}} & =0 .
\end{aligned}
$$

Furthermore, we have a smaller, 8-dimensional version of this,

$$
A_{0}^{\text {perm }}:=\mathfrak{s p}(1)[2] \oplus \mathfrak{s p}(1)[1] \oplus \mathbb{R}[1] \oplus \mathbb{R},
$$

which has the same operations as above, only the two $S_{0}^{2} \mathfrak{s p}(1)$-components are missing. There is a natural projection $A^{\text {perm }} \rightarrow A_{0}^{\text {perm }}$.

From these definitions, we immediately obtain:

2.2.52 Lemma. Given a hyperkähler manifold with permuting Sp(1)-action, we obtain a natural morphism of $S p(1)^{\star}$-modules

$$
A^{\text {perm }} \rightarrow \Omega^{*}(M)
$$

Its image is the $S p(1)^{\star}$-module generated by $\omega_{1}$ (or equivalently $\omega_{1}, \omega_{2}, \omega_{3}$ ). It factors through $A_{0}^{\text {perm }}$ if and only if $\rho_{2}=0$. 
2.2.53 Remark. Note that the morphism $A^{\text {perm }} \rightarrow \Omega^{*}(M)$ is injective if $\rho_{2} \neq 0$. And if $\rho_{2}=0$, the morphism $A_{0}^{\text {perm }} \rightarrow \Omega^{*}(M)$ is injective. This follows from Lemma 2.2.41 and Proposition 2.2.44 and the $S p(1)$-equivariance of the morphisms.

2.2.54 Remark. Similarly, one can define a $\operatorname{Spin}_{\varepsilon}^{G}(3)^{\star}$-module structure on

$$
A_{G}^{\text {perm }}:=A^{\text {perm }} \oplus(\mathfrak{g} \otimes \mathfrak{s p}(1))[1] \oplus(\mathfrak{g} \otimes \mathfrak{s p}(1)),
$$

and, given a hyperkähler manifold $M$ with permuting $\operatorname{Spin}_{\varepsilon}^{G}(3)$-action, obtain a degreepreserving morphism of $\operatorname{Spin}_{\varepsilon}^{G}(3)^{\star}$-modules $A_{G}^{\text {perm }} \rightarrow \Omega^{*}(M)$, whose image is the $\operatorname{Spin}_{\varepsilon}^{G}(3)^{\star}$ module generated by $\omega_{1}$ (or, equivalently, $\omega_{1}, \omega_{2}, \omega_{3}$ ).

2.2.55 Remark. Alternatively, the $S p(1)^{\star}$-module $A^{\text {perm }}$ can be described as quotients of the universal enveloping algebra $U(\widetilde{\mathfrak{s p}(1)})$ : Consider the left ideal $I \subset U(\widetilde{\mathfrak{s p}(1)})$ generated by

$d, \mathcal{L}_{\zeta_{1}}, \mathcal{L}_{\zeta_{2}} \mathcal{L}_{\zeta_{3}}, \iota_{1} \iota_{2} \iota_{3}, \mathcal{L}_{\zeta_{2}}^{2}+4, \mathcal{L}_{\zeta_{3}}^{2}+4, \iota_{\zeta_{1}} \iota_{\zeta_{3}} \mathcal{L}_{\zeta_{2}}-\iota_{\zeta_{2}} \iota_{\zeta_{1}} \mathcal{L}_{\zeta_{3}}, \iota_{\zeta_{1}} \iota_{\zeta_{2}}-\frac{1}{2} \iota_{\zeta_{3}} \iota_{\zeta_{2}} \mathcal{L}_{\zeta_{2}}, \iota_{\zeta_{1}} \iota_{\zeta_{3}}+\frac{1}{2} \iota_{\zeta_{2}} \iota_{\zeta_{3}} \mathcal{L}_{\zeta_{3}}$

It follows from Example 2.2.11 and the super version of the Poincaré-Birkhoff-Witt theorem ([MM65, Thm. 5.15]) that

$$
A^{\text {perm }}=U(\widetilde{\mathfrak{s p}(1)}) / I
$$

and a real basis is given by

$$
\begin{aligned}
& \omega_{1}, \iota_{\zeta_{1}} \omega_{1}, \iota_{\zeta_{2}} \omega_{1}, \iota_{\zeta_{3}} \omega_{1}, \\
& \mathcal{L}_{\zeta_{2}} \omega_{1}, \mathcal{L}_{\zeta_{3}} \omega_{1}, \iota_{\zeta_{1}} \mathcal{L}_{\zeta_{2}} \omega_{1}, \iota_{\zeta_{2}} \mathcal{L}_{\zeta_{2}} \omega_{1}, \iota_{\zeta_{3}} \mathcal{L}_{\zeta_{2}} \omega_{1}, \iota_{\zeta_{1}} \mathcal{L}_{\zeta_{3}} \omega_{1}, \iota_{\zeta_{2}} \mathcal{L}_{\zeta_{3}} \omega_{1}, \iota_{\zeta_{3}} \mathcal{L}_{\zeta_{3}} \omega_{1}, \\
& \iota_{\zeta_{1}} \iota_{\zeta_{2}} \omega_{1}, \iota_{\zeta_{1}} \iota_{\zeta_{3}} \omega_{1}, \iota_{\zeta_{2}} \iota_{\zeta_{3}} \omega_{1}, \iota_{\zeta_{1}} \iota_{\zeta_{2}} \mathcal{L}_{\zeta_{2}} \omega_{1}, \iota_{\zeta_{1}} \iota_{\zeta_{3}} \mathcal{L}_{\zeta_{2}} \omega_{1}, \iota_{\zeta_{1}} \iota_{\zeta_{3}} \mathcal{L}_{\zeta_{3}} \omega_{1} .
\end{aligned}
$$

Similarly, we have

$$
A_{0}^{\text {perm }}=U(\widetilde{\mathfrak{s p}(1)}) / I^{\prime}
$$

where $I^{\prime}$ is the left ideal generated by $I$ and the additional generators

$$
\begin{aligned}
& \iota_{\zeta_{1}} \iota_{\zeta_{2}}, \iota_{\zeta_{1}} \iota_{\zeta_{3}}, 2 \iota_{\zeta_{3}}-\iota_{\zeta_{1}} \mathcal{L}_{\zeta_{2}}, 2 \iota_{\zeta_{1}}+\iota_{\zeta_{3}} \mathcal{L}_{\zeta_{2}} \\
& 2 \iota_{\zeta_{2}}+\iota_{\zeta_{1}} \mathcal{L}_{\zeta_{3}}, 2 \iota_{\zeta_{1}}+\iota_{\zeta_{2}} \mathcal{L}_{\zeta_{3}}, \iota_{\zeta_{2}} \mathcal{L}_{\zeta_{2}}-\iota_{\zeta_{3}} \mathcal{L}_{\zeta_{3}}, 2 \iota_{\zeta_{2}} \iota_{\zeta_{3}}+\iota_{\zeta_{1}} \iota_{\zeta_{2}} \mathcal{L}_{\zeta_{2}}
\end{aligned}
$$

and a real basis of $A_{0}^{\text {perm }}$ is given by

$$
\omega_{1}, \iota_{\zeta_{1}} \omega_{1}, \iota_{\zeta_{2}} \omega_{1}, \iota_{\zeta_{3}} \omega_{1}, \mathcal{L}_{\zeta_{2}} \omega_{1}, \mathcal{L}_{\zeta_{3}} \omega_{1}, \iota_{\zeta_{2}} \iota_{\zeta_{3}} \omega_{1}, \iota_{\zeta_{2}} \mathcal{L}_{\zeta_{2}} \omega_{1}
$$

2.2.56 Remark. We will also study an analogue of $\sum_{\ell=0}^{2}\left(\iota_{\mathfrak{s p i n}_{\varepsilon}^{G}(3)}\right)^{\ell} \omega$, where $\omega$ is replaced by the fundamental 4 -form $\Omega=\operatorname{tr}(\omega \wedge \omega)$, in chapter 3 . 


\section{Chapter 3}

\section{Homotopy moment maps and equivariant cohomology}

In this chapter we study the notion of homotopy moment maps, which generalize moment maps in symplectic geometry to the case of Lie group actions on manifolds preserving a closed $n+1$-form, called pre-n-plectic form. We are particularly interested in the case of $n=3$, i.e. manifolds with a closed 4 -form. As an example, we construct homotopy moment maps for tri-hamiltonian as well as permuting actions on hyperkähler manifolds equipped with the fundamental 4 -form $\Omega$. These are obtained from the cocylces of degree 4 in equivariant cohomology constructed in Theorem 2.2.22. We generalize this construction and show that cocycles of arbitrary degree in equivariant cohomology give rise to homotopy moment maps. This generalizes the interpretation of moment maps in terms of equivariant cohomology given by Atiyah-Bott ([AB84]).

Work on this section started after discussions with Christopher L. Rogers and Marco Zambon, after Marco Zambon gave a talk on homotopy moment maps in the "Higher Structures" seminar in Göttingen, shortly after the first version of their joint paper [FRZ13] with Yaël Frégier appeared on the arXiv. The results of section 3.3 also appear in the second version of the same paper [CFRZ15]. The author is grateful to Yaël Frégier, Christopher L. Rogers and Marco Zambon for allowing him to join their project at such a late stage, and in particular to Christopher L. Rogers for helpful discussions and hints.

Throughout, $G$ will be a Lie group with Lie algebra $\mathfrak{g}$ and $M$ a $G$-manifold.

\subsection{Homotopy moment maps}

3.1.1 Definition. Let $M$ be a manifold, $\Omega \in \Omega^{n+1}(M)$ closed and $G \curvearrowright M$ a smooth action which preserves $\Omega$. A homotopy moment map $f=\sum_{k=1}^{n} f_{k}$ for $(M, \Omega)$ consists of $f_{k} \in \Lambda^{k}\left(\mathfrak{g}^{\vee}\right) \otimes \Omega^{n-k}(M), k=1, \ldots n$ satisfying

$$
\delta_{\mathfrak{g}} f+d f=-\sum_{k=1}^{n+1} \zeta(k) \iota_{\mathfrak{g}}^{k} \Omega,
$$


where $\zeta(k):=-(-1)^{\frac{k(k+1)}{2}}, \delta_{\mathfrak{g}}$ is the Chevalley-Eilenberg differential and $d$ is the exterior derivative.

A homotopy moment maps $f$ is said to be $G$-equivariant if $f_{1}, \ldots f_{n}$ are $G$-invariant.

3.1.2 Remark. The origin of this definition is the following: Associated to $(M, \Omega)$ is a Lie- $n$-algebra of observables $\operatorname{Ham}_{\infty}(M, \Omega)$, which generalizes the Poisson Lie algebra of a symplectic manifold. A homotopy moment map $f$ is the same as an $L_{\infty}$-morphism $\mathfrak{g} \rightarrow \operatorname{Ham}_{\infty}(M, \Omega)$, lifting the infinitesimal $G$-action by hamiltonian vector fields. This generalizes the interpretation of a (co)moment map in symplectic geometry as a lift of the infinitesimal $G$-action to the Poisson Lie algebra. More details on this point of view can be found in [CFRZ15], in particular in Def./Prop. 5.1. For our purposes, it will be sufficient to work with the above definition.

The Lie $n$-algebra $L_{\infty}(M, \Omega)$, of which $\operatorname{Ham}_{\infty}(M, \Omega)$ is a sighly modified version, was first constructed in $[\operatorname{Rog} 12]$.

3.1.3 Notation. We will also use $\tilde{f}:=\sum_{k=1}^{n} \zeta(k) f_{k}$. In terms of $\tilde{f}$, the moment map condition for $f$ reads

$$
d_{\mathfrak{g}} \tilde{f}=F^{\Omega},
$$

where $F^{\Omega}:=\sum_{k=1}^{n+1}(-1)^{k+1} \iota_{\mathfrak{g}}^{k} \Omega$ and $d_{\mathfrak{g}}:=\delta_{\mathfrak{g}}+(-1)^{k} d$ is the differential on the total complex $C^{*}(\mathfrak{g}, M)$ of the double complex

$$
C^{k, m}(\mathfrak{g}, M):=\bigwedge^{k}\left(\mathfrak{g}^{\vee}\right) \otimes \Omega^{m}(M) .
$$

This complex computes the Lie algebra cohomology of $\mathfrak{g}$ with values in the trivial $\mathfrak{g}$ module $\Omega^{*}(M)$. A similar complex which computes the Lie algebra homology with values in the $\mathfrak{g}$-module $\Omega^{*}(M)$ has been studied by Brylinski in [Bry90]. The interpretation of homotopy moment maps in terms the complex $C^{*}(\mathfrak{g}, M)$ has also been studied in [FLGZ14].

3.1.4 Remark. If we interpret $F^{\Omega}: \wedge^{*}\left(\mathfrak{g}^{\vee}\right) \rightarrow \Omega^{*}(M)$, then the image of $F^{\Omega}$ and $\Omega$ linearly span the $G^{\star}$-submodule of $\Omega^{*}(M)$ generated by $\Omega$.

3.1.5 Remark. Note that if $f=\sum_{k=1}^{n} f_{k}$ is a homotopy moment map for a pre- $n$-plectic action of $G$ on $(M, \Omega)$ (i.e. $\left.\Omega \in \Omega^{n+1}(M)^{G}\right)$, then the restriction of $-\tilde{f}_{n}$ to ker $\delta_{\mathfrak{g}} \subset \wedge^{n} \mathfrak{g}^{\vee}$ is a multi-moment map in the sense of Madsen and Swann ([MS12], [MS13]).

3.1.6 Example $(\boldsymbol{n}=3)$. Since one of our main interests is the case of pre-3-plectic manifolds $(M, \Omega)$, i.e. $\Omega$ is a closed 4 -form, we explicitly write out the moment map conditions in this case: A homotopy moment map $f$ consists of

- $f_{1} \in \mathfrak{g}^{\vee} \otimes \Omega^{2}(M)$,

- $f_{2} \in \wedge^{2} \mathfrak{g}^{\vee} \otimes \Omega^{1}(M)$

- $f_{3} \in \wedge^{3} \mathfrak{g}^{\vee} \otimes \Omega^{0}(M)$, 
satisfying the following conditions for all $\xi_{1}, \xi_{2}, \xi_{3}, \xi_{4} \in \mathfrak{g}$ :

$$
\begin{aligned}
d f_{1}\left(\xi_{1}\right)+\iota_{v_{\xi_{1}}^{G}} \Omega= & 0 \\
d f_{2}\left(\xi_{1}, \xi_{2}\right)+\iota_{v_{\xi_{2}}} \iota_{\xi_{\xi_{1}}^{G}} \Omega= & f_{1}\left(\left[\xi_{1}, \xi_{2}\right]\right), \\
d f_{3}\left(\xi_{1}, \xi_{2}, \xi_{3}\right)-\iota_{\xi_{\xi_{3}}^{G}} \iota_{v_{\xi_{2}}} \iota_{v_{\xi_{1}}^{G}} \Omega= & f_{2}\left(\left[\xi_{1}, \xi_{2}\right], \xi_{3}\right)-f_{2}\left(\left[\xi_{1}, \xi_{3}\right], \xi_{2}\right) \\
& +f_{2}\left(\left[\xi_{2}, \xi_{3}\right], \xi_{1}\right), \\
-\iota_{v_{\xi_{4}}^{G}} \iota_{v_{\xi_{3}}^{G}} \iota_{v_{\xi_{2}}^{G}} \iota_{v_{\xi_{1}}^{G}} \Omega= & f_{3}\left(\left[\xi_{1}, \xi_{2}\right], \xi_{3}, \xi_{4}\right)-f_{3}\left(\left[\xi_{1}, \xi_{3}\right], \xi_{2}, \xi_{4}\right) \\
& +f_{3}\left(\left[\xi_{1}, \xi_{4}\right], \xi_{2}, \xi_{3}\right)+f_{3}\left(\left[\xi_{2}, \xi_{3}\right], \xi_{1}, \xi_{4}\right) \\
& -f_{3}\left(\left[\xi_{2}, \xi_{4}\right], \xi_{1}, \xi_{3}\right)+f_{3}\left(\left[\xi_{3}, \xi_{4}\right], \xi_{1}, \xi_{2}\right) .
\end{aligned}
$$

\subsection{Homotopy moment maps from degree 4 cocycles in the Cartan model}

In this section we construct explicit homotopy moment maps from degree 4 cocycles in the Cartan model for equivariant cohomology and apply this to the cocycles constructed in Theorem 2.2.22 for actions on hyperkähler manifolds.

Even though the first part of the following Theorem 3.2.1 is a special case of the more general Theorem 3.3.27 below, we still give an independet, direct proof, without using the other models for equivariant cohomology. The second part of the theorem compares the homotopy moment maps constructed from two degree 4 cocycles which differ by a $d_{G^{-}}$exact form. The first part of this theorem seemed to be well-known to the experts, and the author would like to thank C. Rogers and M. Zambon for sharing the formula for the moment map in this case. The second part is an immediate consequence of the first part.

\subsubsection{Theorem.}

1. Every cocycle $\bar{\Omega}=\Omega+P_{1}+P_{2} \in C_{G}^{4}(M)$ in the Cartan model with $\Omega \in \Omega^{4}(M)^{G}$, $P_{1} \in\left(\mathfrak{g}^{\vee} \otimes \Omega^{2}(M)\right)^{G}$ and $P_{2} \in\left(S^{2}\left(\mathfrak{g}^{\vee}\right) \otimes \Omega^{0}(M)\right)^{G}$ induces a (G-equivariant) homotopy moment map $f$ with

$$
\begin{aligned}
& f_{1}:=-P_{1}, \\
& f_{2}:=\iota_{\mathfrak{g}} f_{1}+d P_{2}=\pi_{\wedge^{2} \mathfrak{g} \vee} \iota_{\mathfrak{g}} f_{1}, \\
& f_{3}:=\iota_{\mathfrak{g}} f_{2}+\left(\mathrm{id}_{\mathfrak{g} \vee} \otimes \delta_{\mathfrak{g}}\right) P_{2}=-\pi_{\wedge^{3} \mathfrak{g} \vee} \iota_{\mathfrak{g}}^{2} f_{1}+\pi_{\wedge^{3} \mathfrak{g} \vee}\left(\operatorname{id}_{\mathfrak{g} \vee} \otimes \delta_{\mathfrak{g}}\right) P_{2} .
\end{aligned}
$$

2. Let $\alpha \in\left(\mathfrak{g}^{\vee} \otimes \Omega^{1}(M)\right)^{G}$ and $\beta \in \Omega^{3}(M)^{G}$. Then the homotopy moment map constructed from the 2 -step extension $\Omega+P_{1}+P_{2}+d_{G}(\alpha+\beta)$ of $\Omega+d \beta$ is

$$
\begin{aligned}
& f_{1}^{\prime}=f_{1}-\iota_{\mathfrak{g}} \beta+d \alpha, \\
& f_{2}^{\prime}=f_{2}-\iota_{\mathfrak{g}}^{2} \beta-\pi_{\wedge^{2} \mathfrak{g} \vee} \iota_{\mathfrak{g}} d \alpha, \\
& f_{3}^{\prime}=f_{3}-\iota_{\mathfrak{g}}^{3} \beta-\delta_{\mathfrak{g}} \pi_{\wedge^{2} \mathfrak{g} \vee} \iota_{\mathfrak{g}} \alpha,
\end{aligned}
$$

where $f$ is the homotopy moment map constructed from $\Omega+P_{1}+P_{2}$. 
3.2.2 Remark. The second part of Theorem 3.2.1 generalizes [FLGZ14, Prop. 7.11]. In Corollary 3.3.31 below, we generalize this to arbitrary to cocycles of arbitrary degree.

Proof (of Theorem 3.2.1).

1. Before proving that the conditions (3.3-3.6) hold, we need to check that $f_{2}$ and $f_{3}$ is actually skew-symmetic. For $f_{2}$, this is obvious from $f_{2}=\iota_{\mathfrak{g}} f_{1}+d P_{2}=$ $\iota_{\mathfrak{g}} f_{1}-\pi_{S^{2} \mathfrak{g} \vee} \iota_{\mathfrak{g}} f_{1}=\pi_{\wedge^{2} \mathfrak{g} \vee} \iota_{\mathfrak{g}} f_{1}$. From the definition of $f_{3}$, we see immediately that $f_{3}\left(\xi_{1}, \xi_{2}, \xi_{3}\right)=-f_{3}\left(\xi_{1}, \xi_{3}, \xi_{2}\right)$. Furthermore,

$$
\begin{aligned}
f_{3}\left(\xi_{1}, \xi_{2}, \xi_{3}\right) & =-\iota_{\mathfrak{g}}^{2} f_{1}\left(\xi_{1}, \xi_{2}, \xi_{3}\right)+\mathcal{L}_{\mathfrak{g}} P_{2}\left(\xi_{1}, \xi_{2}, \xi_{3}\right)-P_{2}\left(\xi_{1},\left[\xi_{2}, \xi_{3}\right]\right) \\
& =-\iota_{\mathfrak{g}}^{2} f_{1}\left(\xi_{1}, \xi_{2}, \xi_{3}\right)+P_{2}\left(\left[\xi_{1}, \xi_{2}\right], \xi_{3}\right)+P_{2}\left(\xi_{2},\left[\xi_{1}, \xi_{3}\right]\right)-P_{2}\left(\xi_{1},\left[\xi_{2}, \xi_{3}\right]\right) \\
& =\iota_{\mathfrak{g}}^{2} f_{1}\left(\xi_{2}, \xi_{1}, \xi_{3}\right)-\mathcal{L}_{\mathfrak{g}} P_{2}\left(\xi_{2}, \xi_{1}, \xi_{3}\right)+P_{2}\left(\xi_{2},\left[\xi_{1}, \xi_{3}\right]\right) \\
& =-f_{3}\left(\xi_{2}, \xi_{1}, \xi_{3}\right) .
\end{aligned}
$$

Hence $f_{3} \in \bigwedge^{3} \mathfrak{g}^{\vee} \otimes \Omega^{0}(M)$ as claimed.

The cocycle condition in the Cartan model is $d \Omega=0, d f_{1}=-d P_{1}=-\iota_{\mathfrak{g}} \Omega$ and $d P_{2}=\pi_{S^{2} \mathfrak{g} \vee} \iota_{\mathfrak{g}} P_{1}$. The second of these is already (3.3). To check the second condition (3.4), we compute

$$
\begin{aligned}
f_{1}\left(\left[\xi_{1}, \xi_{2}\right]\right) & =\mathcal{L}_{\mathfrak{g}} f_{1}\left(\xi_{1} \otimes \xi_{2}\right)=\left(\iota_{\mathfrak{g}} d+d \iota_{\mathfrak{g}}\right) f_{1}\left(\xi_{1} \otimes \xi_{2}\right) \\
& =-\iota_{\mathfrak{g}} \iota_{\mathfrak{g}} \Omega\left(\xi_{1} \otimes \xi_{2}\right)+d \iota_{\mathfrak{g}} f_{1}\left(\xi_{1} \otimes \xi_{2}\right)
\end{aligned}
$$

Since the left hand side and $\iota_{\mathfrak{g}} \iota_{\mathfrak{g}} \Omega\left(\xi_{1} \otimes \xi_{2}\right)$ are skew-symmetric in $\xi_{1}, \xi_{2}$, we have $f_{1}\left(\left[\xi_{1}, \xi_{2}\right]\right)=-\iota_{v_{\xi_{1}}} \iota_{v_{\xi_{2}}} \Omega+d \pi_{\wedge^{2} \mathfrak{g} \vee} \iota_{\mathfrak{g}} f_{1}\left(\xi_{1} \otimes \xi_{2}\right)=\iota_{v_{\xi_{2}}} \iota_{v_{\xi_{1}}} \Omega+d f_{2}\left(\xi_{1} \otimes \xi_{2}\right)$.

Using $f_{2}=\iota_{\mathfrak{g}} f_{1}+d P_{2}$ and Equation 3.4, we compute

$$
\begin{aligned}
-\delta_{\mathfrak{g}} f_{2}\left(\xi_{1}, \xi_{2}, \xi_{3}\right) & =\mathcal{L}_{\mathfrak{g}} f_{2}\left(\xi_{1}, \xi_{2}, \xi_{3}\right)-f_{2}\left(\xi_{1},\left[\xi_{2}, \xi_{3}\right]\right) \\
& =\mathcal{L}_{\mathfrak{g}} f_{2}\left(\xi_{1}, \xi_{2}, \xi_{3}\right)-\iota_{\mathfrak{g}} f_{1}\left(\xi_{1},\left[\xi_{2}, \xi_{3}\right]\right)-d P_{2}\left(\xi_{1},\left[\xi_{2}, \xi_{3}\right]\right) \\
& =\mathcal{L}_{\mathfrak{g}} f_{2}\left(\xi_{1}, \xi_{2}, \xi_{3}\right)+\iota_{\mathfrak{g}} \delta_{\mathfrak{g}} f_{1}\left(\xi_{1}, \xi_{2}, \xi_{3}\right)-d P_{2}\left(\xi_{1},\left[\xi_{2}, \xi_{3}\right]\right) \\
& =\mathcal{L}_{\mathfrak{g}} f_{2}\left(\xi_{1}, \xi_{2}, \xi_{3}\right)-\iota_{\mathfrak{g}} d f_{2}\left(\xi_{1}, \xi_{2}, \xi_{3}\right)-\iota_{\mathfrak{g}}^{3} \Omega\left(\xi_{1}, \xi_{2}, \xi_{3}\right)-d P_{2}\left(\xi_{1},\left[\xi_{2}, \xi_{3}\right]\right) \\
& =d \iota_{\mathfrak{g}} f_{2}\left(\xi_{1}, \xi_{2}, \xi_{3}\right)-d P_{2}\left(\xi_{1},\left[\xi_{2}, \xi_{3}\right]\right)-\iota_{\mathfrak{g}}^{3} \Omega\left(\xi_{1}, \xi_{2}, \xi_{3}\right) \\
& =d f_{3}\left(\xi_{1}, \xi_{2}, \xi_{3}\right)-\iota_{\mathfrak{g}}^{3} \Omega\left(\xi_{1}, \xi_{2}, \xi_{3}\right) .
\end{aligned}
$$

This proves (3.5). We now turn our attention to the last condition (3.6). We first observe that

$$
\begin{aligned}
\delta_{\mathfrak{g}} f_{2}\left(\xi_{2}, \xi_{3}, \xi_{4}\right) & =f_{2}\left(-\left(\left[\xi_{2}, \xi_{3}\right], \xi_{4}\right)+\left(\left[\xi_{2}, \xi_{4}\right], \xi_{3}\right)-\left(\left[\xi_{3}, \xi_{4}\right], \xi_{1}\right)\right) \\
& =\left(\iota_{\mathfrak{g}} f_{1}+d P_{2}\right)([\cdot, \cdot] \otimes \mathrm{id})\left(-\left(\xi_{2}, \xi_{3}, \xi_{4}\right)+\left(\xi_{2}, \xi_{4}, \xi_{3}\right)-\left(\xi_{3}, \xi_{4}, \xi_{2}\right)\right)
\end{aligned}
$$


We use this to show $-\delta_{\mathfrak{g}} f_{3}-\mathcal{L}_{\mathfrak{g}} f_{3}=\iota_{\mathfrak{g}} \delta_{\mathfrak{g}} f_{2}$ :

$$
\begin{aligned}
& \left(-\delta_{\mathfrak{g}} f_{3}-\mathcal{L}_{\mathfrak{g}} f_{3}\right)\left(\xi_{1}, \ldots, \xi_{4}\right) \\
= & f_{3}\left(\left[\xi_{2}, \xi_{3}\right], \xi_{1}, \xi_{4}\right)-f_{3}\left(\left[\xi_{2}, \xi_{4}\right], \xi_{1}, \xi_{3}\right)+f_{3}\left(\left[\xi_{3}, \xi_{4}\right], \xi_{1}, \xi_{2}\right) \\
= & \iota_{\mathfrak{g}}^{2} f_{1}\left(\left(\xi_{1},\left[\xi_{2}, \xi_{3}\right], \xi_{4}\right)-\left(\xi_{1},\left[\xi_{2}, \xi_{4}\right], \xi_{3}\right)+\left(\xi_{1},\left[\xi_{3}, \xi_{4}\right], \xi_{2}\right)\right) \\
& -\mathcal{L}_{\mathfrak{g}} P_{2}\left(\left(\xi_{1},\left[\xi_{2}, \xi_{3}\right], \xi_{4}\right)-\left(\xi_{1},\left[\xi_{2}, \xi_{4}\right], \xi_{3}\right)+\left(\xi_{1},\left[\xi_{3}, \xi_{4}\right], \xi_{2}\right)\right) \\
& +P_{2}\left(\left(\xi_{1},\left[\left[\xi_{2}, \xi_{3}\right], \xi_{4}\right]\right)-\left(\xi_{1},\left[\left[\xi_{2}, \xi_{4}\right], \xi_{3}\right]\right)+\left(\xi_{1},\left[\left[\xi_{3}, \xi_{4}\right], \xi_{2}\right]\right)\right) \\
= & \iota_{\mathfrak{g}} \delta_{\mathfrak{g}} f_{2}\left(\xi_{1}, \ldots, \xi_{4}\right) \\
& -\left((\mathrm{id} \otimes[\cdot, \cdot] \otimes \mathrm{id})^{\vee} \iota_{\mathfrak{g}} d P_{2}\right)\left(-\left(\xi_{1}, \xi_{2}, \xi_{3}, \xi_{4}\right)+\left(\xi_{1}, \xi_{2}, \xi_{4}, \xi_{3}\right)-\left(\xi_{1}, \xi_{3}, \xi_{4}, \xi_{2}\right)\right) \\
& -\left((\mathrm{id} \otimes[\cdot, \cdot] \otimes \mathrm{id})^{\vee} \mathcal{L}_{\mathfrak{g}} P_{2}\right)\left(\left(\xi_{1}, \xi_{2}, \xi_{3}, \xi_{4}\right)-\left(\xi_{1}, \xi_{2}, \xi_{4}, \xi_{3}\right)+\left(\xi_{1}, \xi_{3}, \xi_{4}, \xi_{2}\right)\right) \\
= & \iota_{\mathfrak{g}} \delta_{\mathfrak{g}} f_{2}\left(\xi_{1}, \ldots, \xi_{4}\right) .
\end{aligned}
$$

To finally prove the last condition (3.6), we again use the equivariance of $f_{3}$ and condition (3.5) to compute:

$$
-\delta_{\mathfrak{g}} f_{3}=\mathcal{L}_{\mathfrak{g}} f_{3}+\iota_{\mathfrak{g}} \delta_{\mathfrak{g}} f_{2}=\mathcal{L}_{\mathfrak{g}} f_{3}+\iota_{\mathfrak{g}}\left(-d f_{3}+\iota_{\mathfrak{g}}^{3} \Omega\right)=\mathcal{L}_{\mathfrak{g}} f_{3}-\mathcal{L}_{\mathfrak{g}} f_{3}-\iota_{\mathfrak{g}}^{4} \Omega=-\iota_{\mathfrak{g}}^{4} \Omega .
$$

2. We have $\Omega+P_{1}+P_{2}+d_{G}(\alpha+\beta)=\Omega^{\prime}+P_{1}^{\prime}+P_{2}^{\prime}$ is a 2-step extension of $\Omega^{\prime}:=\Omega+d \beta$ with $P_{1}^{\prime}:=P_{1}-\iota_{\mathfrak{g}} \beta+d \alpha$ and $P_{2}^{\prime}:=P_{2}-\pi_{S^{2} \mathfrak{g} \vee} \iota_{\mathfrak{g}} \alpha$. In particular, we have

$$
\begin{aligned}
& f_{1}^{\prime}=-P_{1}^{\prime}=-P_{1}+\iota_{\mathfrak{g}} \beta-d \alpha=f_{1}+\iota_{\mathfrak{g}} \beta-d \alpha, \\
& f_{2}^{\prime}=\pi_{\wedge^{2} \mathfrak{g} \vee} \iota_{\mathfrak{g}} f_{1}^{\prime}=\pi_{\wedge^{2} \mathfrak{g} \vee} \iota_{\mathfrak{g}} f_{1}+\pi_{\wedge^{2} \mathfrak{g} \vee}\left(\iota_{\mathfrak{g}}\right)^{2} \beta-\pi_{\wedge^{2} \mathfrak{g} \vee} \iota_{\mathfrak{g}} d \alpha=f_{2}-\iota_{\mathfrak{g}}^{2} \beta-\pi_{\wedge^{2} \mathfrak{g} \vee} \iota_{\mathfrak{g}} d \alpha,
\end{aligned}
$$

Before turning to $f_{3}^{\prime}$, we observe that

$$
\begin{aligned}
\mathcal{L}_{\mathfrak{g}} \pi_{\wedge^{2} \mathfrak{g} \vee} \vee \iota_{\mathfrak{g}} \alpha\left(\xi_{1}, \xi_{2}, \xi_{3}\right) & =\frac{1}{2}\left(\mathcal{L}_{v_{\xi_{1}}^{G} \iota_{\xi_{2}}} \alpha\left(\xi_{3}\right)-\mathcal{L}_{v_{\xi_{1}}^{G}} \iota_{\xi_{\xi_{3}}^{G}} \alpha\left(\xi_{2}\right)\right) \\
& =\frac{1}{2}\left(\iota_{v_{\left[\xi_{1}, \xi_{2}\right]}^{G}} \alpha\left(\xi_{3}\right)+\iota_{v_{\xi_{2}}^{G}} \alpha\left(\left[\xi_{1}, \xi_{3}\right]\right)-\iota_{v_{\left[\xi_{1}, \xi_{3}\right]}} \alpha\left(\xi_{2}\right)-\iota_{v_{\xi_{3}}^{G}} \alpha\left(\left[\xi_{1}, \xi_{2}\right]\right)\right) \\
& =\pi_{\wedge^{2} \mathfrak{g} \vee \iota_{\mathfrak{g}}} \alpha\left(\left(\left[\xi_{1}, \xi_{2}\right], \xi_{3}\right)-\left(\left[\xi_{1}, \xi_{3}\right], \xi_{2}\right)\right),
\end{aligned}
$$

and, therefore,

$$
\begin{aligned}
& \left(\left(\operatorname{id}_{\mathfrak{g} \vee} \otimes \delta_{\mathfrak{g}}\right) \pi_{\wedge^{2} \mathfrak{g} \vee} \iota_{\mathfrak{g}} \alpha+\mathcal{L}_{\mathfrak{g}} \pi_{\wedge^{2} \mathfrak{g}^{\vee}} \iota_{\mathfrak{g}} \alpha\right)\left(\xi_{1}, \xi_{2}, \xi_{3}\right) \\
= & \pi_{\wedge^{2} \mathfrak{g} \vee} \iota_{\mathfrak{g}} \alpha\left(\left(\left[\xi_{2}, \xi_{3}\right], \xi_{1}\right)+\left(\left[\xi_{1}, \xi_{2}\right], \xi_{3}\right)-\left(\left[\xi_{1}, \xi_{3}\right], \xi_{2}\right)\right) \\
= & -\delta_{\mathfrak{g}} \pi_{\wedge^{2} \mathfrak{g} \vee} \iota_{\mathfrak{g}} \alpha\left(\xi_{1}, \xi_{2}, \xi_{3}\right) .
\end{aligned}
$$


Using this, we finally compute

$$
\begin{aligned}
f_{3}^{\prime} & =\iota_{\mathfrak{g}} f_{2}^{\prime}+\left(\mathrm{id}_{\mathfrak{g} \vee} \otimes \delta_{\mathfrak{g}}\right) P_{2}^{\prime} \\
& =\iota_{\mathfrak{g}} f_{2}-\iota_{\mathfrak{g}}^{3} \beta-\iota_{\mathfrak{g}} \pi_{\wedge^{2} \mathfrak{g} \vee} \iota_{\mathfrak{g}} d \alpha+\left(\mathrm{id}_{\mathfrak{g} \vee} \otimes \delta_{\mathfrak{g}}\right) P_{2}-\left(\mathrm{id}_{\mathfrak{g} \vee} \otimes \delta_{\mathfrak{g}}\right) \pi_{S^{2} \mathfrak{g} \vee} \iota_{\mathfrak{g}} \alpha \\
& =f_{3}-\iota_{\mathfrak{g}}^{3} \beta-\iota_{\mathfrak{g}} \mathcal{L}_{\mathfrak{g}} \alpha+\iota_{\mathfrak{g}} d \pi_{\wedge^{2} \mathfrak{g} \vee} \iota_{\mathfrak{g}} \alpha-\left(\mathrm{id}_{\mathfrak{g}} \otimes \delta_{\mathfrak{g}}\right) \pi_{S^{2} \mathfrak{g} \vee} \iota_{\mathfrak{g}} \alpha \\
& =f_{3}-\iota_{\mathfrak{g}}^{3} \beta+\left(\mathrm{id}_{\mathfrak{g} \vee} \otimes \delta_{\mathfrak{g}}\right) \iota_{\mathfrak{g}} \alpha+\mathcal{L}_{\mathfrak{g}} \pi_{\wedge^{2} \mathfrak{g} \vee} \iota_{\mathfrak{g}} \alpha-\left(\mathrm{id}_{\mathfrak{g} \vee} \otimes \delta_{\mathfrak{g}}\right) \pi_{S^{2} \mathfrak{g} \vee} \iota_{\mathfrak{g}} \alpha \\
& =f_{3}-\iota_{\mathfrak{g}}^{3} \beta+\left(\mathrm{id}_{\mathfrak{g} \vee} \otimes \delta_{\mathfrak{g}}\right) \pi_{\wedge^{2} \mathfrak{g} \vee} \iota_{\mathfrak{g}} \alpha+\mathcal{L}_{\mathfrak{g}} \pi_{\wedge^{2} \mathfrak{g} \vee} \iota_{\mathfrak{g}} \alpha \\
& =f_{3}-\iota_{\mathfrak{g}}^{3} \beta-\delta_{\mathfrak{g}} \pi_{\wedge^{2} \mathfrak{g} \vee} \iota_{\mathfrak{g}} \alpha
\end{aligned}
$$

3.2.3 Proposition. Let $M$ be a hyperkähler manifold. Each of the 2-step extensions of $\Omega=\operatorname{tr}(\omega \wedge \omega)$ in Theorem 2.2.22 induces a homotopy moment map:

1. If $G \curvearrowright M$ is tri-hamiltonian, then

$$
\begin{aligned}
f_{1}^{G}: & =2 \operatorname{tr}(\mu \otimes \omega), \\
f_{2}^{G}: & =2 \pi_{\wedge^{2} \mathfrak{g} \vee} \operatorname{tr}(\mu \otimes d \mu), \\
f_{3}^{G}: & =3 \pi_{\wedge^{3} \mathfrak{g} \vee}\left(\operatorname{id}_{\mathfrak{g} \vee} \otimes \delta_{\mathfrak{g}}\right) \operatorname{tr}(\mu \otimes \mu)
\end{aligned}
$$

is a homotopy moment map.

If additionally, $\omega=d \gamma$, there is another homotopy moment map

$$
\begin{aligned}
& f_{1}^{\prime G}:=\operatorname{tr}(\mu \otimes \omega), \\
& f_{2}^{\prime G}:=2 \pi_{\wedge^{2} \mathfrak{g} \vee} \operatorname{tr}(\mu \otimes d \mu)+\delta_{\mathfrak{g}} \operatorname{tr}(\gamma \otimes \mu), \\
& f_{3}^{\prime G}:=3 \pi_{\wedge^{3} \mathfrak{g} \vee}\left(\mathrm{id}_{\mathfrak{g} \vee} \otimes \delta_{\mathfrak{g}}\right) \operatorname{tr}(\mu \otimes \mu),
\end{aligned}
$$

which is constructed from the 1-step extension $d_{G} \operatorname{tr}(\gamma \wedge \omega)$.

2. If $S p(1) \curvearrowright M$ is permuting, then

$$
\begin{aligned}
f_{1} & :=-4 \pi_{\mathfrak{s p}(1) \vee}(\gamma \wedge \gamma)-2 \operatorname{tr}_{23}(\rho \otimes \omega), \\
f_{2} & :=2 \pi_{\wedge^{2} \mathfrak{s p}(1) \vee} \operatorname{tr}_{24}\left(\rho \otimes\left(d \rho-2 \delta_{\mathfrak{s p}(1)} \gamma\right)\right), \\
f_{3} & :=2 \operatorname{tr}(\rho)^{2}-4 \operatorname{tr}\left(\rho^{2}\right)
\end{aligned}
$$

is a homotopy moment map (constructed from $\bar{\Omega}$ ). Furthermore,

$$
\begin{aligned}
& f_{1}^{\prime}:=-4 \pi_{\mathfrak{s p}(1)^{\vee}}(\gamma \wedge \gamma)-\operatorname{tr}_{23}(\rho \otimes \omega)-\operatorname{tr}_{13}(\gamma \wedge d \rho), \\
& f_{2}^{\prime}:=\pi_{\wedge^{2} \mathfrak{s p}(1)^{\vee}} \operatorname{tr}_{24}\left(\rho \otimes\left(2 d \rho-3 \delta_{\mathfrak{s p}(1)} \gamma\right)\right)+\pi_{\wedge^{2} \mathfrak{s p}(1) \vee} \operatorname{tr}_{14}\left(\gamma \otimes \mathcal{L}_{\mathfrak{s p}(1)} \rho\right), \\
& f_{3}^{\prime}:=2 \operatorname{tr}(\rho)^{2}-4 \operatorname{tr}\left(\rho^{2}\right)
\end{aligned}
$$

is a homotopy moment map (constructed from $d_{S p(1)} \operatorname{tr}(\gamma \wedge \omega)$ ). 
3. If $S p(1) \times G \curvearrowright M$, the $S p(1)$-action is permuting and the $G$-action is hyperkähler, then $f^{S p(1) \times G}$ is a homotopy moment map:

$$
\begin{aligned}
f_{1}^{S p(1) \times G} & =-4 \pi_{\mathfrak{s p}(1) \vee}(\gamma \wedge \gamma)-2 \operatorname{tr}_{2,3}(\rho \otimes \omega)+2 \operatorname{tr}(\mu \otimes \omega), \\
f_{2}^{S p(1) \times G} & =f_{2}+f_{2}^{G} \\
& +\pi_{\wedge^{2}\left(\mathfrak{s p}(1)^{\vee} \oplus \mathfrak{g}^{\vee}\right)}\left(2 \operatorname{tr}_{23}\left(\left(d \rho-\delta_{\mathfrak{s p}(1)} \gamma\right) \otimes \mu\right)-8 \pi_{\mathfrak{s p}(1) \vee}(\mu \otimes \gamma)+2 \operatorname{tr}_{12}(d \mu \otimes \rho)\right),
\end{aligned}
$$

Finally, $f_{3}^{S p(1) \times G}$ is uniquely determined by its restrictions:

$$
\begin{aligned}
\left.f_{3}^{S p(1) \times G}\right|_{\mathfrak{s p}(1) \otimes 3}= & f_{3}, \\
\left.f_{3}^{S p(1) \times G}\right|_{\mathfrak{g} \otimes 3}= & f_{3}^{G}, \\
\left.f_{3}^{S p(1) \times G}\right|_{\mathfrak{s p}(1) \otimes 2 \otimes \mathfrak{g}}= & \operatorname{tr}_{34}\left(\left(\mathcal{L}_{\mathfrak{s p}(1)} \rho+\left(\operatorname{id}_{\mathfrak{s p}(1)} \otimes \delta_{\mathfrak{s p}(1)}\right) \rho\right) \otimes \mu\right)-4\left(\operatorname{id}_{\mathfrak{s p}(1) \otimes \mathfrak{g}} \otimes \pi_{\mathfrak{s p}(1) \vee}\right)(\rho \otimes \mu) \\
& -\operatorname{tr}_{23}\left(\mathcal{L}_{\mathfrak{s p}(1)} \mu \otimes \rho\right), \\
\left.f_{3}^{S p(1) \times G}\right|_{\mathfrak{s p}(1) \otimes \mathfrak{g} \otimes 2}= & 8 \pi_{\mathfrak{s p}(1) \vee}(\mu \otimes \mu)-\left(\operatorname{id}_{\mathfrak{s p}(1)} \otimes \delta_{\mathfrak{g}}\right) \operatorname{tr}_{23}(\rho \otimes \mu) .
\end{aligned}
$$

Here, we used the isomorphisms

$$
\begin{aligned}
& \bigwedge^{2}\left(\mathfrak{s p}(1)^{\vee} \oplus \mathfrak{g}^{\vee}\right) \cong \bigwedge^{2}\left(\mathfrak{s} \mathfrak{p}(1)^{\vee}\right) \oplus \bigwedge^{2}\left(\mathfrak{g}^{\vee}\right) \oplus \mathfrak{s p}(1)^{\vee} \otimes \mathfrak{g}^{\vee} \\
& \bigwedge^{3}\left(\mathfrak{s p}(1)^{\vee} \oplus \mathfrak{g}^{\vee}\right) \cong \bigwedge^{3}\left(\mathfrak{s} \mathfrak{p}(1)^{\vee}\right) \oplus \bigwedge^{2}\left(\mathfrak{s} \mathfrak{p}(1)^{\vee}\right) \otimes \mathfrak{g}^{\vee} \oplus \mathfrak{s p}(1)^{\vee} \otimes \bigwedge^{2}\left(\mathfrak{g}^{\vee}\right) \oplus \bigwedge^{3}\left(\mathfrak{g}^{\vee}\right) .
\end{aligned}
$$

3.2.4 Remark. In the case of the permuting $S p(1)$-action on a hyperkähler manifold, we can use the decomposition $S^{2}\left(\mathfrak{s p}(1)^{\vee}\right) \cong \mathbb{R} \oplus S_{0}^{2}\left(\mathfrak{s p}(1)^{\vee}\right)$, and the corresponding decomposition of $\rho$ into $\rho_{0}$ and $\rho_{2}$ to write $f_{3}$ in terms of $\rho_{0}$ and $\rho_{2}$ :

$$
f_{3}=f_{3}^{\prime}=6 \rho_{0}^{2}-4 \operatorname{tr}\left(\rho_{2}^{2}\right) .
$$

3.2.5 Remark. After this result was obtained, C. Shahbazi and M. Zambon pointed out an alternative way of constructing a homotopy moment map for a tri-hamiltonian action on a hyperkähler manifold in [SZ15]. Their approach is to first construct homotopy moment maps for wedge powers of $n$-plectic forms and afterwards take sums of homotopy moment maps. Their construction yields a moment map which in general differs from the homotopy moment map obtained in Theorem 3.3.27.

3.2.6 Remark. The third part of the Proposition 3.2.3 provides an explicit moment map for a permuting $\operatorname{Spin}_{\varepsilon}^{G}(m)$-action (Definition 2.2.1) on a hyperkähler manifold $M$.

To show that the explicit formulae for the moment maps in the corollary hold, we first prove the following two lemmas:

3.2.7 Lemma. Let $G \curvearrowright M$ be a tri-hamiltonian action on a hyperkähler manifold. Then

$$
\pi_{\wedge^{3} \mathfrak{g} \vee} \iota_{\mathfrak{g}}^{2} \operatorname{tr}(\mu \otimes \omega)=-\pi_{\wedge^{3} \mathfrak{g} \vee}\left(\mathrm{id}_{\mathfrak{g} \vee} \otimes \delta_{\mathfrak{g}}\right) \operatorname{tr}(\mu \otimes \mu)
$$


Proof. The $G$-invariance of $\mu$ implies

$$
\left(\delta_{\mathfrak{g}} \otimes \operatorname{id}_{\mathfrak{s p}(1)^{\vee}}\right) \mu=-\mathcal{L}_{\mathfrak{g}} \mu=-\iota_{\mathfrak{g}} d \mu=\iota_{\mathfrak{g}} \iota_{\mathfrak{g}} \omega .
$$

Using this, we compute

$$
\begin{aligned}
-\operatorname{tr}(\mu \otimes \mu)\left(\xi_{1} \otimes\left[\xi_{2}, \xi_{3}\right]\right) & =\operatorname{tr}\left(\mu \otimes \iota_{\mathfrak{g}} \iota_{\mathfrak{g}} \omega\right)\left(\xi_{1} \otimes \xi_{2} \otimes \xi_{3}\right) \\
& =\iota_{\mathfrak{g}} \iota_{\mathfrak{g}} \operatorname{tr}(\mu \otimes \omega)\left(\xi_{2} \otimes \xi_{3} \otimes \xi_{1}\right) .
\end{aligned}
$$

Skew-symmetrizing in $\xi_{1}, \xi_{2}, \xi_{3}$ gives the claimed identity.

3.2.8 Lemma. Let $S p(1) \curvearrowright M$ be a permuting action on a hyperkähler manifold. Then the following equalities hold:

1. $\iota_{\mathfrak{s p}(1)} \iota_{\mathfrak{s p}(1)} \omega=-\left(\mathrm{id}_{\mathfrak{s p}(1) \vee} \otimes \delta_{\mathfrak{s p}(1)}\right) \rho+\mathcal{L}_{\mathfrak{s p}(1)} \rho$,

2. $\pi_{\wedge^{3} \mathfrak{s p}(1)^{\vee}} \iota_{\mathfrak{s p}(1)} \iota_{\mathfrak{s p}(1)} \operatorname{tr}_{2,3}(\rho \otimes \omega)=\left(\frac{4}{3} \operatorname{tr}\left(\rho^{2}\right)-\frac{2}{3} \operatorname{tr}(\rho)^{2}\right) v o l_{\mathfrak{s p}(1)}$,

3. $\pi_{\wedge^{3} \mathfrak{s p}(1) \vee} \iota_{\mathfrak{s p}(1)} \iota_{\mathfrak{s p}(1)} \pi_{\mathfrak{s p}(1) \vee}(\gamma \wedge \gamma)=\frac{1}{6}\left(\operatorname{tr}\left(\rho^{2}\right)-\operatorname{tr}(\rho)^{2}\right) v o l_{\mathfrak{s p}(1)}$,

4. $\pi_{\wedge^{3} \mathfrak{s p}(1) \vee}\left(\operatorname{id}_{\mathfrak{s p}(1) \vee} \otimes \delta\right) \rho^{2}=-\frac{2}{3} \operatorname{tr}\left(\rho^{2}\right) v o l_{\mathfrak{s p}(1)}$,

5. $\iota_{\mathfrak{s p}(1)} \operatorname{tr}_{13}(\gamma \wedge d \rho)=-\operatorname{tr}_{24}(\rho \otimes d \rho)-\operatorname{tr}_{14}\left(\gamma \otimes \mathcal{L}_{\mathfrak{s p}(1)} \rho\right)$,

6. $\pi_{\wedge^{3} \mathfrak{s p}(1) \vee} \iota_{\mathfrak{s p}(1)} \iota_{\mathfrak{s p}(1)} \operatorname{tr}_{13}(\gamma \wedge d \rho)=\left(\left(2 \operatorname{tr}\left(\rho^{2}\right)-\frac{2}{3} \operatorname{tr}(\rho)^{2}\right) \operatorname{vol}_{\mathfrak{s p}(1)}\right.$.

Here, $\operatorname{vol}_{\mathfrak{s p}(1)}$ denotes the standard volume form on $\mathfrak{s p}(1) \cong \mathbb{R}^{3}$.

Proof.

1. $\iota_{\mathfrak{s p}(1)} \iota_{\mathfrak{s p}(1)} \omega=\iota_{\mathfrak{s p}(1)}\left(-\delta_{\mathfrak{s p}(1)} \gamma+d \rho\right)=-\left(\operatorname{id}_{\mathfrak{s p}(1) \vee} \otimes \delta_{\mathfrak{s p}(1)}\right) \rho+\mathcal{L}_{\mathfrak{s p}(1)} \rho$.

In particular, using the $S p(1)$-invariance of $\rho$, we have

$$
\left(\mathcal{L}_{\mathfrak{s p}(1)} \rho\right)\left(\xi_{1} \otimes \xi_{2} \otimes \xi_{3}\right)=\rho\left(\left[\xi_{1}, \xi_{2}\right] \otimes \xi_{3}\right)+\rho\left(\xi_{2} \otimes\left[\xi_{1}, \xi_{3}\right]\right),
$$

and hence,

$$
\begin{aligned}
& \iota_{v_{j}^{S p(1)}} \iota_{v_{k}^{S p(1)}} \omega_{1}=2(\rho(i \otimes i)-\rho(j \otimes j)-\rho(k \otimes k)), \\
& \iota_{v_{k}^{S p(1)} \iota} v_{i}^{S p(1)} \omega_{2}=2(\rho(j \otimes j)-\rho(i \otimes i)-\rho(k \otimes k)), \\
& \iota_{v_{i}^{S p(1)}} \iota v_{j}^{S p(1)} \omega_{3}=2(\rho(k \otimes k)-\rho(i \otimes i)-\rho(j \otimes j)), \\
& \iota_{v_{j}^{S p(1)} \iota} v_{k}^{S p(1)} \omega_{2}=\iota_{v_{k}^{S p(1)} \iota} v_{i}^{S p(1)} \omega_{1}=4 \rho(i \otimes j), \\
& \iota_{v_{j}^{S p(1)}} \iota v_{k}^{S p(1)} \omega_{3}=\iota_{v_{i}^{S p(1)}} \iota v_{j}^{S p(1)} \omega_{1}=4 \rho(i \otimes k), \\
& \iota_{v_{k}^{S p(1)} \iota} v_{i}^{S p(1)} \omega_{3}=\iota_{v_{i}^{S p(1)}} \iota v_{j}^{S p(1)} \omega_{2}=4 \rho(j \otimes k) .
\end{aligned}
$$


2. Using the previous statement, we have

$$
\begin{aligned}
& \pi_{\wedge^{3} \mathfrak{s p}(1) \vee} \iota_{\mathfrak{s p}(1)} \iota_{\mathfrak{s p}(1)} \operatorname{tr}_{2,3}(\rho \otimes \omega)(i \otimes j \otimes k) \\
& =\pi_{\wedge^{3} \mathfrak{s p}(1) \vee} \operatorname{tr}_{2,5}\left(\rho \otimes \iota_{\mathfrak{s p}(1)} \iota_{\mathfrak{s p}(1)} \omega\right)(i \otimes j \otimes k) \\
& =\frac{1}{3} \operatorname{tr}_{2,5}\left(\rho \otimes \iota_{\mathfrak{s p}(1)} \iota_{\mathfrak{s p}(1)} \omega\right)(i \otimes j \otimes k+j \otimes k \otimes i+k \otimes i \otimes j) \\
& +\rho(j \otimes i) \iota_{v_{k}^{S p(1)}} \iota_{v_{i}^{S p(1)}} \omega_{1}+\rho(j \otimes j) \iota_{v_{k}^{S p(1)}} \iota_{v_{i}^{S p(1)}} \omega_{2}+\rho(j \otimes k) \iota_{v_{k}^{S p(1)}} \iota_{v_{i}^{S p(1)}} \omega_{3} \\
& \left.+\rho(k \otimes i) \iota_{v_{i}^{S p(1)}} \iota_{v_{j}^{S p(1)}} \omega_{1}+\rho(k \otimes j) \iota_{v_{i}^{S p(1)}} \iota_{v_{j}^{S p(1)}} \omega_{2}+\rho(k \otimes k) \iota_{v_{i}^{S p(1)}} \iota_{v_{j}^{S p(1)}} \omega_{3}\right) \\
& =\frac{1}{3}\left(2 \rho(i \otimes i)(\rho(i \otimes i)-\rho(j \otimes j)-\rho(k \otimes k))+4 \rho(i \otimes j)^{2}+4 \rho(i \otimes k)^{2}\right. \\
& +4 \rho(j \otimes i)^{2}+2 \rho(j \otimes j)(\rho(j \otimes j)-\rho(i \otimes i)-\rho(k \otimes k))+4 \rho(j \otimes k)^{2} \\
& \left.+4 \rho(k \otimes i)^{2}+4 \rho(k \otimes j)^{2}+2 \rho(k \otimes k)(\rho(k \otimes k)-\rho(i \otimes i)-\rho(j \otimes j))\right) \\
& =\frac{2}{3} \sum_{\ell} \rho\left(\zeta_{\ell} \otimes \zeta_{\ell}\right)^{2}-\frac{2}{3} \sum_{\ell \neq m} \rho\left(\zeta_{\ell} \otimes \zeta_{\ell}\right) \rho\left(\zeta_{m} \otimes \zeta_{m}\right)+\frac{4}{3} \sum_{\ell \neq m} \rho\left(\zeta_{\ell} \otimes \zeta_{m}\right)^{2} \\
& =-\frac{2}{3} \sum_{\ell, m} \rho\left(\zeta_{\ell} \otimes \zeta_{\ell}\right) \rho\left(\zeta_{m} \otimes \zeta_{m}\right)+\frac{4}{3} \sum_{\ell, m} \rho\left(\zeta_{\ell} \otimes \zeta_{m}\right)^{2} \\
& =\frac{4}{3} \operatorname{tr}\left(\rho^{2}\right)-\frac{2}{3} \operatorname{tr}(\rho)^{2},
\end{aligned}
$$

where, as before, we use the notation $\zeta_{1}:=i, \zeta_{2}:=j, \zeta_{3}:=k$.

3. For $\xi_{1}, \xi_{2}, \xi_{3} \in \mathfrak{s p}(1)$ we have

$$
\begin{aligned}
& \iota_{\mathfrak{s p}(1)} \iota_{\mathfrak{s p}(1)} \pi_{\mathfrak{s p}(1) \vee}(\gamma \wedge \gamma)\left(\xi_{1} \otimes \xi_{2} \otimes \xi_{3}\right) \\
= & -2 \iota_{\mathfrak{s p}(1)}\left(\operatorname{id}_{\mathfrak{s p}(1)^{\vee}} \otimes \pi_{\mathfrak{s p}(1)^{\vee}}\right)(\rho \otimes \gamma)\left(\xi_{1} \otimes \xi_{2} \otimes \xi_{3}\right) \\
= & -2 \rho\left(\xi_{2} \otimes \cdot\right) \iota_{v_{\xi_{1}}^{S p(1)}} \gamma\left(\pi_{\mathfrak{s p}(1) \vee}^{\vee}\left(\xi_{3}\right)\right) \\
= & 2 \rho\left(\xi_{2} \otimes \cdot\right) \rho\left(\xi_{1} \otimes \cdot\right)\left(\pi_{\mathfrak{s p}(1)^{\vee}}^{\vee}\left(\xi_{3}\right)\right) \\
= & 2\left(\operatorname{id}_{\mathfrak{s p}(1)^{\vee}} \otimes \pi_{\mathfrak{s p}(1)^{\vee}} \otimes \operatorname{id}_{\mathfrak{s p}(1) \vee}\right)(\rho \otimes \rho)\left(\xi_{1} \otimes \xi_{3} \otimes \xi_{2}\right) .
\end{aligned}
$$

Skew-symmetrizing this and evaluating on $i \otimes j \otimes k$ gives

$$
\begin{aligned}
& \pi_{\wedge^{3} \mathfrak{s p}(1)^{\vee} l_{\mathfrak{s p}(1)} \iota_{\mathfrak{s p}(1)} \pi_{\mathfrak{s p}(1)^{\vee}}(\gamma \wedge \gamma)(i \otimes j \otimes k)} \\
= & -\frac{1}{3}\left(\left(\operatorname{id}_{\mathfrak{s p}(1)^{\vee}} \otimes \pi_{\mathfrak{s p}(1)^{\vee}} \otimes \operatorname{id}_{\mathfrak{s p}(1)^{\vee}}\right)(\rho \otimes \rho)\right)(i \otimes j \otimes k+j \otimes k \otimes i+k \otimes i \otimes j \\
& -i \otimes k \otimes j-j \otimes i \otimes k-k \otimes j \otimes i) \\
= & \frac{1}{6}(\rho(j \otimes i) \rho(i \otimes j)-\rho(j \otimes j) \rho(i \otimes i)+\rho(k \otimes j) \rho(j \otimes k)-\rho(k \otimes k) \rho(j \otimes j) \\
& +\rho(i \otimes k) \rho(k \otimes i)-\rho(i \otimes i) \rho(k \otimes k)-\rho(k \otimes k) \rho(i \otimes i)+\rho(k \otimes i) \rho(i \otimes k) \\
& -\rho(i \otimes i) \rho(j \otimes j)+\rho(i \otimes j) \rho(j \otimes i)-\rho(j \otimes j) \rho(k \otimes k)+\rho(j \otimes k) \rho(k \otimes j)) \\
= & \frac{1}{6}\left(\sum_{\ell, m=1}^{3}\left(\rho\left(\zeta_{\ell} \otimes \zeta_{m}\right)^{2}-\rho\left(\zeta_{\ell} \otimes \zeta_{\ell}\right) \rho\left(\zeta_{m} \otimes \zeta_{m}\right)\right)\right. \\
= & \frac{1}{6}\left(\operatorname{tr}\left(\rho^{2}\right)-\operatorname{tr}(\rho)^{2}\right)
\end{aligned}
$$


4. We have

$$
\begin{aligned}
& \pi_{\wedge^{3} \mathfrak{s p}(1)^{\vee}}\left(\operatorname{id}_{\mathfrak{s p}(1)^{\vee}} \otimes \delta_{\mathfrak{s p}(1)}\right) \rho^{2}(i \otimes j \otimes k) \\
= & \frac{1}{3}\left(\operatorname{id}_{\mathfrak{s p}(1)^{\vee}} \otimes \delta_{\mathfrak{s p}(1)}\right) \rho^{2}(i \otimes j \otimes k+j \otimes k \otimes i+k \otimes i \otimes j) \\
= & -\frac{2}{3} \rho^{2}(i \otimes i+j \otimes j+k \otimes k) \\
= & -\frac{2}{3} \operatorname{tr}\left(\rho^{2}\right)
\end{aligned}
$$

and hence, $\pi_{\wedge^{3} \mathfrak{s p}(1) \vee}\left(\mathrm{id}_{\mathfrak{s p}(1)^{\vee}} \otimes \delta_{\mathfrak{s p}(1)}\right) \rho^{2}=-\frac{2}{3} \operatorname{tr}\left(\rho^{2}\right) v o l_{\mathfrak{s p}(1)}$.

5. $\iota_{\mathfrak{s p}(1)} \operatorname{tr}_{13}(\gamma \wedge d \rho)=\operatorname{tr}_{24}\left(\iota_{\mathfrak{s p}(1)}(\gamma \wedge d \rho)\right)=-\operatorname{tr}_{24}(\rho \otimes d \rho)-\operatorname{tr}_{14}\left(\gamma \otimes \mathcal{L}_{\mathfrak{s p}(1)} \rho\right)$.

6. For $\xi_{1}, \xi_{2}, \xi_{3} \in \mathfrak{s p}(1)$, we have

$$
\begin{aligned}
& \iota_{\mathfrak{s p}(1)} \iota_{\mathfrak{s p}(1)} \operatorname{tr}_{13}(\gamma \wedge d \rho)\left(\xi_{1} \otimes \xi_{2} \otimes \xi_{3}\right) \\
= & -\left(\iota_{\mathfrak{s p}(1)} \operatorname{tr}_{24}(\rho \otimes d \rho)+\iota_{\mathfrak{s p}(1)} \operatorname{tr}_{14}\left(\gamma \otimes \mathcal{L}_{\mathfrak{s p}(1)} \rho\right)\right)\left(\xi_{1} \otimes \xi_{2} \otimes \xi_{3}\right) \\
= & -\left(\operatorname{tr}_{35}\left(\iota_{\mathfrak{s p}(1)}(\rho \otimes d \rho)\right)+\operatorname{tr}_{25}\left(\iota_{\mathfrak{s p}(1)} \gamma \otimes \mathcal{L}_{\mathfrak{s p}(1)} \rho\right)\right)\left(\xi_{1} \otimes \xi_{2} \otimes \xi_{3}\right) \\
= & -\operatorname{tr}\left(\rho\left(\xi_{2} \otimes \cdot\right) \mathcal{L}_{v_{\xi_{1}}^{S p(1)}} \rho\left(\xi_{3} \otimes \cdot\right)+\operatorname{tr}\left(\rho\left(\xi_{1} \otimes \cdot\right) \mathcal{L}_{v_{\xi_{2}}^{S p(1)}} \rho\left(\xi_{3} \otimes \cdot\right)\right.\right. \\
= & 2\left(\pi_{\wedge^{2} \mathfrak{s p}(1) \vee} \otimes \operatorname{id}_{\mathfrak{s p}(1) \vee}\right) \operatorname{tr}_{25}\left(\rho \otimes \mathcal{L}_{\mathfrak{s p}(1)} \rho\right)\left(\xi_{1} \otimes \xi_{2} \otimes \xi_{3}\right) .
\end{aligned}
$$

In particular, we have $\pi_{\wedge^{3} \mathfrak{s p}(1) \vee} \iota_{\mathfrak{s p}(1)} \iota_{\mathfrak{s p}(1)} \operatorname{tr}_{13}(\gamma \wedge d \rho)=2 \pi_{\wedge^{3} \mathfrak{s p}(1)^{\vee}} \operatorname{tr}_{25}\left(\rho \otimes \mathcal{L}_{\mathfrak{s p}(1)} \rho\right)$. Using this, we can compute

$$
\begin{aligned}
& \pi_{\wedge^{3} \mathfrak{s p}(1) \vee} \iota_{\mathfrak{s p}(1)} \iota_{\mathfrak{s p}(1)} \operatorname{tr}_{13}(\gamma \wedge d \rho)(i \otimes j \otimes k) \\
& =\frac{1}{3} \operatorname{tr}_{25}\left(\rho \otimes \mathcal{L}_{\mathfrak{s p}(1)} \rho\right)(i \otimes j \otimes k+j \otimes k \otimes i+k \otimes i \otimes j-k \otimes j \otimes i-j \otimes i \otimes k-i \otimes k \otimes j) \\
& =\frac{1}{3} \sum_{\ell=1}^{3}\left(\rho\left(i \otimes \zeta_{\ell}\right) \rho\left([j, k] \otimes \zeta_{\ell}\right)+\rho\left(i \otimes \zeta_{\ell}\right) \rho\left(k,\left[j, \zeta_{\ell}\right]\right)\right. \\
& +\rho\left(j \otimes \zeta_{\ell}\right) \rho\left([k, i] \otimes \zeta_{\ell}\right)+\rho\left(j \otimes \zeta_{\ell}\right) \rho\left(i,\left[k, \zeta_{\ell}\right]\right) \\
& +\rho\left(k \otimes \zeta_{\ell}\right) \rho\left([i, j] \otimes \zeta_{\ell}\right)+\rho\left(k \otimes \zeta_{\ell}\right) \rho\left(j,\left[i, \zeta_{\ell}\right]\right) \\
& -\rho\left(k \otimes \zeta_{\ell}\right) \rho\left([j, i] \otimes \zeta_{\ell}\right)-\rho\left(k \otimes \zeta_{\ell}\right) \rho\left(i,\left[j, \zeta_{\ell}\right]\right) \\
& -\rho\left(i \otimes \zeta_{\ell}\right) \rho\left([k, j] \otimes \zeta_{\ell}\right)-\rho\left(i \otimes \zeta_{\ell}\right) \rho\left(j,\left[k, \zeta_{\ell}\right]\right) \\
& \left.-\rho\left(j \otimes \zeta_{\ell}\right) \rho\left([i, k] \otimes \zeta_{\ell}\right)-\rho\left(j \otimes \zeta_{\ell}\right) \rho\left(k,\left[i, \zeta_{\ell}\right]\right)\right) \\
& =\frac{4}{3} \sum_{\ell=1}^{3}\left(\rho\left(i \otimes \zeta_{\ell}\right)^{2}+\rho\left(j \otimes \zeta_{\ell}\right)^{2}+\rho\left(k \otimes \zeta_{\ell}\right)^{2}\right) \\
& +\frac{2}{3}\left(-\rho(i \otimes i) \rho(k \otimes k)+\rho(i \otimes k)^{2}-\rho(i \otimes i) \rho(j \otimes j)+\rho(i \otimes j)^{2}\right. \\
& +\rho(j \otimes i)^{2}-\rho(j \otimes j) \rho(i \otimes i)-\rho(j \otimes j) \rho(k \otimes k)+\rho(j \otimes k)^{2} \\
& \left.+\rho(j \otimes k)^{2}-\rho(k \otimes k) \rho(j \otimes j)+\rho(k \otimes i)^{2}-\rho(k \otimes k) \rho(i \otimes i)\right) \\
& =\frac{4}{3} \sum_{\ell, \ell^{\prime}=1}^{3} \rho\left(\zeta_{\ell} \otimes \zeta_{\ell^{\prime}}\right)^{2}+\frac{2}{3} \sum_{\ell, \ell^{\prime}=1}^{3}\left(\rho\left(\zeta_{\ell} \otimes \zeta_{\ell^{\prime}}\right)^{2}-\rho\left(\zeta_{\ell} \otimes \zeta_{\ell}\right) \rho\left(\zeta_{\ell^{\prime}} \otimes \zeta_{\ell^{\prime}}\right)\right) \\
& =2 \sum_{\ell, \ell^{\prime}=1}^{3} \rho\left(\zeta_{\ell} \otimes \zeta_{\ell^{\prime}}\right)^{2}-\frac{2}{3} \sum_{\ell, \ell^{\prime}=1}^{3} \rho\left(\zeta_{\ell} \otimes \zeta_{\ell}\right) \rho\left(\zeta_{\ell^{\prime}} \otimes \zeta_{\ell^{\prime}}\right) \\
& =2 \operatorname{tr}\left(\rho^{2}\right)-\frac{2}{3} \operatorname{tr}(\rho)^{2} \text {. }
\end{aligned}
$$


Proof (of Proposition 3.2.3). In each of the cases, we can apply either Theorem 3.2.1 or the more general Theorem 3.3.27 below. They both produce the same homotopy moment map. More precisely, given a cocycle of the form $\Omega+P_{1}+P_{2} \in C_{G}^{4}(M)$, then

$$
\begin{aligned}
& f_{1}=-P_{1}, \\
& f_{2}=-\pi_{\wedge^{2} \mathfrak{g} \vee} \iota_{\mathfrak{g}} P_{1}, \\
& f_{3}=\pi_{\wedge^{3} \mathfrak{g} \vee} \iota_{\mathfrak{g}}^{2} P_{1}+\pi_{\wedge^{3} \mathfrak{g} \vee}\left(\operatorname{id}_{\mathfrak{g} \vee} \otimes \delta_{\mathfrak{g}}\right) P_{2}
\end{aligned}
$$

is a homotopy moment map. We compute these explicitly in the case of the cocycles from Theorem 3.2.1:

1. For the 2-step extension $\Omega-2 \operatorname{tr}(\mu \otimes \omega)+\operatorname{tr}(\mu \otimes \mu) \in C_{G}^{4}(M)$, we have $P_{1}^{G}=$ $-2 \operatorname{tr}(\mu \otimes \omega)$ and $P_{2}^{G}=\operatorname{tr}(\mu \otimes \mu)$. Using Lemma 3.2.7, we obtain

$$
\begin{aligned}
f_{1}^{G} & =-P_{1}^{G}=2 \operatorname{tr}(\mu \otimes \omega), \\
f_{2}^{G} & =-\pi_{\wedge^{2} \mathfrak{g} \vee} \iota_{\mathfrak{g}} P_{1}^{G}=2 \pi_{\wedge^{2} \mathfrak{g} \vee} \iota_{\mathfrak{g}} \operatorname{tr}(\mu \otimes \omega)=-2 \pi_{\wedge^{2} \mathfrak{g} \vee} \operatorname{tr}\left(\mu \otimes \iota_{\mathfrak{g}} \omega\right)=2 \pi_{\wedge^{2} \mathfrak{g} \vee} \operatorname{tr}(\mu \otimes d \mu), \\
f_{3}^{G} & =\pi_{\wedge^{3} \mathfrak{g} \vee} \iota_{\mathfrak{g}}^{2} P_{1}^{G}+\pi_{\wedge^{3} \mathfrak{g} \vee}\left(\mathrm{id}_{\mathfrak{g} \vee} \otimes \delta_{\mathfrak{g}}\right) P_{2}^{G} \\
& =-2 \pi_{\wedge^{3} \mathfrak{g} \vee} \iota_{\mathfrak{g}}^{2} \operatorname{tr}(\mu \otimes \omega)+\pi_{\wedge^{3} \mathfrak{g} \vee}\left(\operatorname{id}_{\mathfrak{g} \vee} \otimes \delta_{\mathfrak{g}}\right) \operatorname{tr}(\mu \otimes \mu) \\
& =3 \pi_{\wedge^{3} \mathfrak{g} \vee}\left(\operatorname{id}_{\mathfrak{g} \vee} \otimes \delta_{\mathfrak{g}}\right) \operatorname{tr}(\mu \otimes \mu) .
\end{aligned}
$$

For the 1-step extension $\Omega-\operatorname{tr}(\mu \otimes \omega)-\operatorname{tr}(\gamma \wedge d \mu) \in C_{G}^{4}(M)$, we have $P_{1}^{\prime G}=$ $-\operatorname{tr}(\mu \otimes \omega)-\operatorname{tr}(\gamma \wedge d \mu)$ and $P_{2}^{\prime G}=0$, and hence

$$
\begin{aligned}
f_{1}^{\prime G} & =-P_{1}^{\prime G}=\operatorname{tr}(\mu \otimes \omega)+\operatorname{tr}(\gamma \wedge d \mu), \\
f_{2}^{\prime G} & =-\pi_{\wedge^{2} \mathfrak{g} \vee} \iota_{\mathfrak{g}} P_{1}^{\prime G}=\pi_{\wedge^{2} \mathfrak{g} \vee} \iota_{\mathfrak{g}} \operatorname{tr}(\mu \otimes \omega)+\pi_{\wedge^{2} \mathfrak{g} \vee} \iota_{\mathfrak{g}} \operatorname{tr}(\gamma \wedge d \mu) \\
& =2 \pi_{\wedge^{2} \mathfrak{g} \vee} \operatorname{tr}(\mu \otimes d \mu)-\pi_{\wedge^{2} \mathfrak{g} \vee} \operatorname{tr}\left(\gamma \otimes \mathcal{L}_{\mathfrak{g}} \mu\right)=2 \pi_{\wedge^{2} \mathfrak{g} \vee} \operatorname{tr}(\mu \otimes d \mu)+\delta_{\mathfrak{g}} \operatorname{tr}(\gamma \otimes \mu), \\
f_{3}^{\prime G} & =\pi_{\wedge^{3} \mathfrak{g} \vee} \iota_{\mathfrak{g}}^{2} P_{1}^{G}=\pi_{\wedge^{3} \mathfrak{g} \vee} \iota_{\mathfrak{g}} f_{2}=2 \pi_{\wedge^{3} \mathfrak{g} \vee} \iota_{\mathfrak{g}} \operatorname{tr}(\mu \otimes d \mu)+2 \pi_{\wedge^{3} \mathfrak{g} \vee} \iota_{\mathfrak{g}} \delta_{\mathfrak{g}} \operatorname{tr}(\gamma \otimes \mu) \\
& =3 \pi_{\wedge^{3} \mathfrak{g} \vee}\left(\mathrm{id}_{\mathfrak{g} \vee} \otimes \delta_{\mathfrak{g}}\right) \operatorname{tr}(\mu \otimes \mu) .
\end{aligned}
$$

2. The claimed formula for $f_{2}$ also follows from the first and second identity in Lemma 2.2.21 and $4 \pi_{\mathfrak{s p}(1)^{\vee}}=-\operatorname{tr}_{13}\left(\operatorname{id}_{\mathfrak{s p}(1) \vee} \otimes \delta_{\mathfrak{s p}(1)}\right)$ :

$$
\begin{aligned}
f_{2} & =-4 \pi_{\wedge^{2} \mathfrak{s p}(1)^{\vee}} \iota_{\mathfrak{s p}(1)} \pi_{\mathfrak{s p}(1) \vee}(\gamma \wedge \gamma)-2 \pi_{\wedge^{2} \mathfrak{s p}(1)^{\vee} \iota_{\mathfrak{s p}(1)}} \operatorname{tr}_{23}(\rho \otimes \omega) \\
& =8 \pi_{\wedge^{2} \mathfrak{s p}(1) \vee}\left(\operatorname{id}_{\mathfrak{s p}(1)^{\vee}} \otimes \pi_{\mathfrak{s p}(1)^{\vee}}\right)(\rho \otimes \gamma)+2 \pi_{\wedge^{2} \mathfrak{s p}(1)^{\vee}} \operatorname{tr}_{2,4}\left(\rho \otimes\left(-\delta_{\mathfrak{s p}(1)} \gamma+d \rho\right)\right) \\
& =2 \pi_{\wedge^{2} \mathfrak{s p}(1) \vee} \operatorname{tr}_{2,4}\left(\rho \otimes\left(d \rho-2 \delta_{\mathfrak{s p}(1)} \gamma\right)\right) .
\end{aligned}
$$

Furthermore, using the identities (2) - (4) from Lemma 3.2.8, we obtain

$$
\begin{aligned}
f_{3} & =\pi_{\wedge^{3} \mathfrak{s p}(1) \vee} \iota_{\mathfrak{s p}(1)} \iota_{\mathfrak{s p}(1)} f_{1}+\pi_{\wedge^{3} \mathfrak{s p}(1) \vee}\left(\operatorname{id}_{\mathfrak{s p}(1) \vee} \otimes \delta_{\mathfrak{s p}(1)}\right) \rho^{2} \\
& =\left(-4\left(\frac{1}{6} \operatorname{tr}\left(\rho^{2}\right)-\frac{1}{6} \operatorname{tr}(\rho)^{2}\right)-2\left(\frac{4}{3} \operatorname{tr}\left(\rho^{2}\right)-\frac{2}{3} \operatorname{tr}(\rho)^{2}\right)-\frac{2}{3} \operatorname{tr}\left(\rho^{2}\right)\right) \operatorname{vol}_{\mathfrak{s p}(1)} \\
& =\left(2 \operatorname{tr}(\rho)^{2}-4 \operatorname{tr}\left(\rho^{2}\right)\right) v o l_{\mathfrak{s p}(1)}
\end{aligned}
$$


For the 1-step extension $d_{S p(1)} \operatorname{tr}(\gamma \wedge \omega)$, we have $P_{1}^{\prime}=4 \pi_{\mathfrak{s p}(1) \vee}(\gamma \wedge \gamma)+\operatorname{tr}_{23}(\rho \otimes$ $\omega)+\operatorname{tr}_{12}(\gamma \wedge d \rho)$ and $P_{2}^{\prime}=0$. Hence, using the last two identities from Lemma 3.2.8

$$
\begin{aligned}
& f_{1}^{\prime}=-P_{1}^{\prime}=-4 \pi_{\mathfrak{s p}(1) \vee}(\gamma \wedge \gamma)-\operatorname{tr}_{23}(\rho \otimes \omega)-\operatorname{tr}_{12}(\gamma \wedge d \rho), \\
& f_{2}^{\prime}=-\pi_{\wedge^{2} \mathfrak{s p}(1)^{\vee}} \iota_{\mathfrak{s p}(1)} P_{1}^{\prime} \\
& =\pi_{\wedge^{2} \mathfrak{s p}(1) \vee}\left(-4 \iota_{\mathfrak{s p}(1)} \pi_{\mathfrak{s p}(1)^{\vee}}(\gamma \wedge \gamma)-\iota_{\mathfrak{s p}(1)} \pi_{\wedge^{2} \mathfrak{s p}(1) \vee} \operatorname{tr}_{23}(\rho \otimes \omega)-\iota_{\mathfrak{s p}(1)} \operatorname{tr}(\gamma \wedge d \rho)\right) \\
& =8 \pi_{\wedge^{2} \mathfrak{s p}(1) \vee}\left(\mathrm{id}_{\mathfrak{s p}(1) \vee} \otimes \pi_{\mathfrak{s p}(1) \vee}\right)(\rho \otimes \gamma)+\pi_{\wedge^{2} \mathfrak{s p}(1) \vee} \operatorname{tr}_{24}\left(\rho \otimes\left(d \rho-\delta_{\mathfrak{s p}(1)} \gamma\right)\right) \\
& +\pi_{\wedge^{2} \mathfrak{s p}(1) \vee} \operatorname{tr}_{24}(\rho \otimes d \rho)+\pi_{\wedge^{2} \mathfrak{s p}(1) \vee} \operatorname{tr}_{14}\left(\gamma \otimes \mathcal{L}_{\mathfrak{s p}(1)} \rho\right) \\
& =\pi_{\wedge^{2} \mathfrak{s p}(1) \vee} \operatorname{tr}_{24}\left(\rho \otimes\left(2 d \rho-3 \delta_{\mathfrak{s p}(1)} \gamma\right)\right)+\pi_{\wedge^{2} \mathfrak{s p}(1) \vee} \operatorname{tr}_{24}\left(\gamma \otimes \mathcal{L}_{\mathfrak{s p}(1)} \rho\right), \\
& f_{3}^{\prime}=\pi_{\wedge^{3} \mathfrak{s p}(1) \vee} \iota_{\mathfrak{s p}(1)} \iota_{\mathfrak{s p}(1)} f_{1}^{\prime} \\
& =\left(-\frac{2}{3}\left(\operatorname{tr}\left(\rho^{2}\right)-\operatorname{tr}(\rho)^{2}\right) \operatorname{vol}_{\mathfrak{s p}(1)}+\left(\frac{2}{3} \operatorname{tr}(\rho)^{2}-\frac{4}{3} \operatorname{tr}\left(\rho^{2}\right)\right) \operatorname{vol}_{\mathfrak{s p}(1)}\right. \\
& +\left(\frac{2}{3} \operatorname{tr}\left(\rho^{2}\right)-2 \operatorname{tr}\left(\rho^{2}\right)\right) v_{\text {ool }}(1) \\
& =\left(2 \operatorname{tr}(\rho)^{2}-4 \operatorname{tr}\left(\rho^{2}\right)\right) \operatorname{vol}_{\mathfrak{s p}(1)} \text {. }
\end{aligned}
$$

3. Since $P_{1}^{S p(1) \times G}=P_{1}+P_{1}^{G}$, we obtain $f_{1}^{S p(1) \times G}=f_{1}+f_{1}^{G}$. The restrictions fo the skew-symmetric part of $\iota_{\mathfrak{s p}(1) \oplus \mathfrak{g}} f_{1}^{S p(1) \times G}$ are

$$
\begin{gathered}
\left.\pi_{\wedge^{2}\left(\mathfrak{s p}(1)^{\vee} \oplus \mathfrak{g}^{\vee}\right)}\left(\iota_{\mathfrak{s p}(1) \oplus \mathfrak{g}} f_{1}^{S p(1) \times G}\right)\right|_{\mathfrak{s p}(1) \otimes \mathfrak{s p}(1)}=\pi_{\wedge^{2}\left(\mathfrak{s p}(1)^{\vee}\right) \iota_{\mathfrak{s p}(1)}} f_{1}^{S p(1)} \\
\left.\pi_{\wedge^{2}\left(\mathfrak{s p}(1)^{\vee} \oplus \mathfrak{g}^{\vee}\right)}\left(\iota_{\mathfrak{s p}(1) \oplus \mathfrak{g}} f_{1}^{S p(1) \times G}\right)\right|_{\mathfrak{g} \otimes \mathfrak{g}}=\pi_{\wedge^{2}\left(\mathfrak{g}^{\vee}\right) \iota_{\mathfrak{s p}(1)} f_{1}^{G}}
\end{gathered}
$$

and

$$
\begin{aligned}
& \left.\pi_{\wedge^{2}\left(\mathfrak{s p}(1)^{\vee} \oplus \mathfrak{g}^{\vee}\right)}\left(\iota_{\mathfrak{s p}(1) \oplus \mathfrak{g}} f_{1}^{S p(1) \times G}\right)\right|_{\mathfrak{s p}(1) \otimes \mathfrak{g}}(\zeta \otimes \xi) \\
= & \frac{1}{2}\left(\left.\iota_{\mathfrak{s p}(1) \oplus \mathfrak{g}} f_{1}^{S p(1) \times G}\right|_{\mathfrak{s p}(1) \otimes \mathfrak{g}}((\zeta, 0) \otimes(0, \xi)-(0, \xi) \otimes(\zeta, 0))\right) \\
= & \frac{1}{2}\left(\iota_{\mathfrak{s p}(1)} f_{1}^{G}(\zeta \otimes \xi)-\iota_{\mathfrak{g}} f_{1}^{S p(1)}(\xi \otimes \zeta)\right) \\
= & \pi_{\wedge^{2}\left(\mathfrak{s p}(1)^{\vee} \oplus \mathfrak{g}^{\vee}\right)}\left(\iota_{\mathfrak{s p}(1)} f_{1}^{G}+\iota_{\mathfrak{g}} f_{1}^{S p(1)}\right)(\zeta, 0) \otimes(0, \xi) .
\end{aligned}
$$

Here, we are using the convention that, for example, $\left.\iota_{\mathfrak{s p}(1)} f_{1}^{G}\right|_{\mathfrak{g} \otimes \mathfrak{s p}(1)}=0$. Since $\Lambda^{2}\left(\mathfrak{s} \mathfrak{p}(1)^{\vee} \oplus \mathfrak{g}^{\vee}\right) \cong \Lambda^{2}\left(\mathfrak{s p}(1)^{\vee}\right) \oplus \mathfrak{s p}(1)^{\vee} \otimes \mathfrak{g}^{\vee} \oplus \Lambda^{2}\left(\mathfrak{g}^{\vee}\right)$, these uniquely determine $f_{2}^{S p(1) \times G}$ :

$f_{2}^{S p(1) \times G}=\pi_{\wedge^{2}\left(\mathfrak{s p}(1)^{\vee} \oplus \mathfrak{g}^{\vee}\right)}\left(\iota_{\mathfrak{s p}(1) \oplus \mathfrak{g}} f_{1}^{S p(1) \times G}\right)=f_{2}+f_{2}^{G}+\pi_{\wedge^{2}\left(\mathfrak{s p}(1)^{\vee} \oplus \mathfrak{g}^{\vee}\right)}\left(\iota_{\mathfrak{s p}(1)} f_{1}^{G}+\iota_{\mathfrak{g}} f_{1}^{S p(1)}\right)$

Since

$$
\iota_{\mathfrak{s p}(1)} f_{1}^{G}=2 \iota_{\mathfrak{s p}(1)} \operatorname{tr}(\omega \otimes \mu)=2 \operatorname{tr}_{23}\left(\iota_{\mathfrak{s p}(1)} \omega \otimes \mu\right)=2 \operatorname{tr}_{23}\left(\left(d \rho-\delta_{\mathfrak{s p}(1)} \gamma\right) \otimes \mu\right)
$$

and

$$
\begin{aligned}
\iota_{\mathfrak{g}} f_{1} & =-4 \iota_{\mathfrak{g}} \pi_{\mathfrak{s p}(1) \vee}(\gamma \wedge \gamma)-2 \iota_{\mathfrak{g}} \operatorname{tr}_{12}(\omega \otimes \rho) \\
& =-8 \pi_{\mathfrak{s p}(1) \vee}(\mu \otimes \gamma)+2 \operatorname{tr}_{12}(\mu \otimes \otimes \rho),
\end{aligned}
$$

the formula for $f_{2}^{S p(1) \times G}$ follows. 
In the case of

$$
\begin{aligned}
& f_{3}^{S p(1) \times G}=\iota_{\mathfrak{s p}(1) \oplus \mathfrak{g}} f_{2}^{S p(1) \times G}+\left(\mathrm{id}_{\mathfrak{s p}(1) \oplus \mathfrak{g}} \otimes \delta_{\mathfrak{s p}(1) \oplus \mathfrak{g})}\right) P_{2}^{S p(1) \times G} \\
& =\iota_{\mathfrak{s p}(1) \oplus \mathfrak{g}}\left(f_{2}^{S p(1)}+f_{2}^{G}+\pi_{\wedge^{2}\left(\mathfrak{s p}(1) \vee \oplus \mathfrak{g}^{\vee}\right)}\left(\iota_{\mathfrak{s p}(1)} f_{1}^{G}+\iota_{\mathfrak{g}} f_{1}\right)\right) \\
& +\left(\operatorname{id}_{\mathfrak{s p}(1) \oplus \mathfrak{g}} \otimes \delta_{\mathfrak{s p}(1) \oplus \mathfrak{g}}\right)\left(P_{2}^{S p(1)}+P_{2}^{G}-\operatorname{tr}_{23}(\rho \otimes \mu),\right.
\end{aligned}
$$

we again have the decomposition

$$
\bigwedge^{3}\left(\mathfrak{s p}(1)^{\vee} \otimes \mathfrak{g}^{\vee}\right) \cong \bigwedge^{3}\left(\mathfrak{s} \mathfrak{p}(1)^{\vee}\right) \oplus \bigwedge^{2}\left(\mathfrak{s} \mathfrak{p}(1)^{\vee}\right) \otimes \mathfrak{g}^{\vee} \oplus \mathfrak{s p}(1)^{\vee} \otimes \bigwedge^{2}\left(\mathfrak{g}^{\vee}\right) \oplus \bigwedge^{3}\left(\mathfrak{g}^{\vee}\right)
$$

Note that using the formula from Theorem 3.2.1, we do not need to skew-symmetrize. In the formula above, we omitted the projections to $\mathfrak{s p}(1) \oplus \mathfrak{g} \rightarrow \mathfrak{s p}(1)$ and $\mathfrak{s p}(1) \oplus \mathfrak{g} \rightarrow$ $\mathfrak{g}$.

The $\Lambda^{3}\left(\mathfrak{s p}(1)^{\vee}\right)$ and $\Lambda^{3}\left(\mathfrak{g}^{\vee}\right)$ components of $f_{3}^{S p(1) \times G}$ are clearly $f_{3}$ and $f_{3}^{G}$, respectively. In the following, we compute the $\Lambda^{2}\left(\mathfrak{s} \mathfrak{p}(1)^{\vee}\right) \otimes \mathfrak{g}^{\vee}$ and $\mathfrak{s p}(1)^{\vee} \otimes \Lambda^{2}\left(\mathfrak{g}^{\vee}\right)$-components by restricting to $\mathfrak{s p}(1) \otimes \mathfrak{s p}(1) \otimes \mathfrak{g}$ and $\mathfrak{s p}(1) \otimes \mathfrak{g} \otimes \mathfrak{g}$, respectively. Together, all these uniquely determine $f_{3}^{S p(1) \times G}$.

$$
\begin{aligned}
& \left.f_{3}^{S p(1) \times G}\right|_{\mathfrak{s p}(1) \otimes 2 \otimes \mathfrak{g}}=\iota_{\mathfrak{s p}(1)} \pi_{\wedge^{2}\left(\mathfrak{s p}(1)^{\vee} \oplus \mathfrak{g}^{\vee}\right.}\left(\iota_{\mathfrak{s p}(1)} f_{1}^{G}+\iota_{\mathfrak{g}} f_{1}\right), \\
& \left.f_{3}^{S p(1) \times G}\right|_{\mathfrak{s p}(1) \otimes \mathfrak{g} \otimes 2}=\iota_{\mathfrak{s p}(1)} f_{2}^{G}-\left(\mathrm{id}_{\mathfrak{s p}(1)} \otimes \delta_{\mathfrak{g}}\right) \operatorname{tr}_{23}(\rho \otimes \mu) .
\end{aligned}
$$

We compute the necessary insertion operations:

$$
\begin{aligned}
& \iota_{\mathfrak{s p}(1)} f_{2}^{G}=-2 \iota_{\mathfrak{s p}(1)} \pi_{\wedge^{2} \mathfrak{g} \vee} \operatorname{tr}(d \mu \otimes \mu) \\
& =-2\left(\operatorname{id}_{\mathfrak{s p}(1)} \otimes \pi_{\wedge 2 \mathfrak{g} \vee}\right) \operatorname{tr}_{23}\left(\mathcal{L}_{\mathfrak{s p}(1)} \mu \otimes \mu\right), \\
& =8 \pi_{\mathfrak{s p}(1) \vee}(\mu \otimes \mu) \text {, } \\
& \left.\iota_{\mathfrak{s p}(1)} \pi_{\wedge^{2}\left(\mathfrak{s p}(1) \vee \oplus \mathfrak{g}^{\vee}\right)} \iota_{\mathfrak{s p}(1)} f_{1}^{G}\right|_{\mathfrak{s p}(1) \otimes 2 \otimes \mathfrak{g}}=\frac{1}{2} \iota_{\mathfrak{s p}(1)} \iota_{\mathfrak{s p}(1)} f_{1}^{G} \\
& =\iota_{\mathfrak{s p}(1)} \operatorname{tr}_{23}\left(\left(d \rho-\delta_{\mathfrak{s p}(1)} \gamma\right) \otimes \mu\right) \\
& =\operatorname{tr}_{34}\left(\left(\mathcal{L}_{\mathfrak{s p}(1)} \rho-\iota_{\mathfrak{s p}(1)} \delta_{\mathfrak{s p}(1)} \gamma\right) \otimes \mu\right) \\
& =\operatorname{tr}_{34}\left(\left(\mathcal{L}_{\mathfrak{s p}(1)} \rho+\left(\operatorname{id}_{\mathfrak{s p}(1)} \otimes \delta_{\mathfrak{s p}(1)}\right) \rho\right) \otimes \mu\right), \\
& \iota_{\mathfrak{s p}(1)} \pi_{\wedge^{2}\left(\mathfrak{s p}(1)^{\vee} \oplus \mathfrak{g}^{\vee}\right)} \iota_{\mathfrak{g}} f_{1}\left(\zeta \otimes \zeta^{\prime} \otimes \xi\right)=-\frac{1}{2} \iota_{\mathfrak{s p}(1)} \iota_{\mathfrak{g}} f_{1}\left(\zeta \otimes \xi \otimes \zeta^{\prime}\right) \\
& =\frac{1}{2} \iota_{\mathfrak{s p}(1)}\left(8 \pi_{\mathfrak{s p}(1) \vee}(\mu \otimes \gamma)-2 \operatorname{tr}_{12}(d \mu \otimes \rho)\right)\left(\zeta \otimes \xi \otimes \zeta^{\prime}\right) \\
& =-4\left(\mathrm{id}_{\mathfrak{s p}(1) \otimes \mathfrak{g}} \otimes \pi_{\mathfrak{s p}(1) \vee}\right)(\rho \otimes \mu)\left(\zeta \otimes \xi \otimes \zeta^{\prime}\right) \\
& -\operatorname{tr}_{23}\left(\mathcal{L}_{\mathfrak{s p}(1)} \mu \otimes \rho\right)\left(\zeta \otimes \xi \otimes \zeta^{\prime}\right) \text {. }
\end{aligned}
$$

Therefore,

$$
\begin{aligned}
\left.f_{3}^{S p(1) \times G}\right|_{\mathfrak{s p}(1) \otimes 2 \otimes \mathfrak{g}}\left(\zeta \otimes \zeta^{\prime} \otimes \xi\right)= & \operatorname{tr}_{34}\left(\left(\mathcal{L}_{\mathfrak{s p}(1)} \rho+\left(\operatorname{id}_{\mathfrak{s p}(1)} \otimes \delta_{\mathfrak{s p}(1)}\right) \rho\right) \otimes \mu\right)\left(\zeta \otimes \zeta^{\prime} \otimes \xi\right) \\
& -4\left(\operatorname{id}_{\mathfrak{s p}(1) \otimes \mathfrak{g}} \otimes \pi_{\left.\mathfrak{s p}(1)^{\vee}\right)(\rho \otimes \mu)\left(\zeta \otimes \xi \otimes \zeta^{\prime}\right)}\right. \\
& -\operatorname{tr}_{23}\left(\mathcal{L}_{\mathfrak{s p}(1)} \mu \otimes \rho\right)\left(\zeta \otimes \xi \otimes \zeta^{\prime}\right)
\end{aligned}
$$

and

$$
\left.f_{3}^{S p(1) \times G}\right|_{\mathfrak{s p}(1) \otimes \mathfrak{g}^{\otimes 2}}=8 \pi_{\mathfrak{s p}(1)^{\vee}}(\mu \otimes \mu)-\left(\mathrm{id}_{\mathfrak{s p}(1)} \otimes \delta_{\mathfrak{g}}\right) \operatorname{tr}_{23}(\rho \otimes \mu)
$$


3.2.9 Remark. Note that the zeros of the homotopy moment map $f^{G}$ in the first part of Proposition 3.2.3 coincide with the zeros of $f_{1}^{G}$, which also coincide with the zeros of the hyperkähler moment map $\mu$. Indeed, $\mu(x)=0$ implies $\left.f_{1}^{G}\right|_{x}=0$ and hence also $\left.f_{2}^{G}\right|_{x}=0$. Furthermore, $\left.\operatorname{tr}(\mu \otimes \mu)\right|_{x}=0$ if and only if $\mu(x)=0$. Conversely, if $\left.f_{1}^{G}\right|_{x}=0$, then $\mu(x)=0$, since $\omega_{1}, \omega_{2}, \omega_{3}$ are linearly independent, nowhere vanishing elements in $\Omega^{2}(M)$.

3.2.10 Remark. Note that if we know $\omega$, we can easily recover $\mu=\frac{1}{2} *\left(f_{1}^{G} \wedge * \omega\right)$ from $f_{1}^{G}$.

3.2.11 Remark. If $G \curvearrowright(M, \Omega)$ is a tri-hamiltonian action of an abelian group, then $f_{3}^{G}=0$. Furthermore, if $G=\mathbb{R}$ or $\mathrm{U}(1)$, then $f_{1}^{G}$ is the only non-vanishing component of the homotopy moment map.

3.2.12 Remark. The analogue of the first part of Proposition 3.2.3 also holds for quaternionic Kähler manifolds $G \curvearrowright(M, \Omega)$ with quaternionic Kähler moment map $\mu$. As explained in Remark 2.2.24, $\Omega-2 \operatorname{tr}_{\mathcal{G}}(\mu \otimes \omega)+\operatorname{tr}_{\mathcal{G}}(\mu \otimes \mu) \in C_{G}^{4}(M)$ is again closed in the Cartan model for $G$-equivariant cohomology and we obtain a homotopy moment map as in the hyperkähler case.

3.2.13 Remark. Note that in the case of a permuting action, the third component of the homotopy moment map constructed from the 2-step extension is equal to the one constructed from the 1 -step extension, i.e. $f_{3}=f_{3}^{\prime}$. The same holds in the case of the tri-hamiltonian action, if the 1-step extension exists. As the second part of Theorem 3.2.1 shows, this is not a coincidence:

Given pre-3-plectic action $G \curvearrowright(M, \Omega)$, i.e. $\Omega \in \Omega^{4}(M)^{G}$ closed, a 2-step extension $\Omega+P_{1}+P_{2}$ and $\alpha \in\left(\mathfrak{g}^{\vee} \otimes \Omega^{1}(M)\right)^{G}$ which satisfies $\iota_{\mathfrak{g}} \alpha=\pi_{S^{2} \mathfrak{g} \vee} \iota_{\mathfrak{g}} \alpha \in\left(S^{2}\left(\mathfrak{g}^{\vee}\right) \otimes \Omega^{0}(M)\right)^{G}$, then the third components of the homotopy moment maps constructed from $\Omega+P_{1}+P_{2}$ and $\Omega+P_{1}+P_{2}+d_{G} \alpha$ agree.

\subsubsection{Examples}

\section{Swann bundles}

Let $M$ be a hyperkähler manifold with permuting $S p(1)$-action and assume $\rho_{2}=0$. Then $\rho=\frac{1}{3} \operatorname{tr}(\rho) \mathbb{1}$, where $\mathbb{1}\left(\zeta_{\ell}, \zeta_{m}\right)=\delta_{\ell, m}, \rho^{2}=\frac{1}{9} \operatorname{tr}(\rho)^{2} \mathbb{1}$ and $\operatorname{tr}\left(\rho^{2}\right)=\frac{1}{3} \operatorname{tr}(\rho)^{2}$.

In this situation, the homotopy moment map from Proposition 3.2.3 is

$$
\begin{aligned}
& f_{1}=-4 \pi_{\mathfrak{s p}(1) \vee}(\gamma \wedge \gamma)-\frac{2}{3} \operatorname{tr}(\rho) \omega=-4 \pi_{\mathfrak{s p}(1) \vee}(\gamma \wedge \gamma)+2 \rho_{0} \omega \\
& f_{2}=\frac{4}{3} \operatorname{tr}(\rho) \delta_{\mathfrak{s p}(1)} \gamma=-4 \rho_{0} \delta_{\mathfrak{s p}(1)} \gamma=-4 \rho_{0} \pi_{\wedge^{2} \mathfrak{s p}(1) \vee} \iota_{\mathfrak{s p}(1)} \omega \\
& f_{3}=\frac{2}{3} \operatorname{tr}(\rho)^{2} \operatorname{vol}_{\mathfrak{s p}(1)}=6 \rho_{0} \operatorname{vol}_{\mathfrak{s p}(1)} .
\end{aligned}
$$

The "reduction" $f_{3}^{-1}(r) / S p(1)$ for $r>0$ is a quaterionic Kähler manifold ([Swa91]). 
Proof.

1. We have $\operatorname{tr}_{2,3}(\rho \otimes \omega)=\frac{1}{3} \operatorname{tr}(\rho) \operatorname{tr}_{2,3}(\mathbb{1} \otimes \omega)=\frac{1}{3} \operatorname{tr}(\rho) \omega$. Hence the formula for $f_{1}$ follows.

2. For $f_{2}$, we have

$$
\begin{aligned}
f_{2} & =2 \pi_{\wedge^{2} \mathfrak{s p}(1) \vee} \operatorname{tr}_{2,4}\left(\rho \otimes\left(-2 \delta_{\mathfrak{s p}(1)} \gamma+d \rho\right)\right) \\
& =\frac{2}{3} \pi_{\wedge^{2} \mathfrak{s p}(1) \vee} \operatorname{tr}_{2,4}\left(\operatorname{tr}(\rho) \mathbb{1} \otimes\left(-2 \delta_{\mathfrak{s p}(1)} \gamma+\frac{1}{3} d \operatorname{tr}(\rho) \mathbb{1}\right)\right) \\
& =-\frac{4}{3} \operatorname{tr}(\rho) \pi_{\wedge^{2} \mathfrak{s p}(1) \vee} \operatorname{tr}_{2,4}\left(\mathbb{1} \otimes \delta_{\mathfrak{s p}(1)} \gamma\right) \\
& =\frac{4}{3} \operatorname{tr}(\rho) \delta_{\mathfrak{s p}(1)} \gamma .
\end{aligned}
$$

Here, we used that $\pi_{\wedge^{2} \mathfrak{s p}(1) \vee} \operatorname{tr}_{2,4}(\mathbb{1} \otimes \mathbb{1})=\pi_{\wedge^{2} \mathfrak{s p}(1) \vee} \mathbb{1}=0$.

3. The formula for $f_{3}$ follows immediately from $\operatorname{tr}\left(\rho^{2}\right)=\frac{1}{3} \operatorname{tr}(\rho)^{2}$.

\section{Quaternionic vector spaces with $S O(3)$-action}

Consider $\mathbb{H}^{n}$ with the permuting action of $S O(3)$ as in Example 2.2.15. Write $x=$ $x_{0}+i x_{1}+j x_{2}+k x_{3}$ with $x_{\ell} \in \mathbb{R}^{n}$. We have $\left.\operatorname{tr}(\rho)\right|_{x}=-3 \rho_{0}(x)=-2\|\operatorname{Im}(x)\|^{2}$. Furthermore,

$$
\begin{aligned}
\left.\operatorname{tr}\left(\rho^{2}\right)\right|_{x}= & 2\|\operatorname{Im}(x)\|^{4}-2\left(\left\|x_{2}\right\|^{2}\left\|x_{3}\right\|^{2}+\left\|x_{1}\right\|^{2}\left\|x_{3}\right\|^{2}+\left\|x_{1}\right\|^{2}\left\|x_{2}\right\|^{2}\right) \\
& +2\left(\left\langle x_{1}, x_{2}\right\rangle^{2}+\left\langle x_{1}, x_{3}\right\rangle^{2}+\left\langle x_{2}, x_{3}\right\rangle^{2}\right) .
\end{aligned}
$$

Hence,

$$
\begin{aligned}
f_{3} & =2 \operatorname{tr}(\rho)^{2}-4 \operatorname{tr}\left(\rho^{2}\right) \\
& =8\left(\left\|x_{2}\right\|^{2}\left\|x_{3}\right\|^{2}+\left\|x_{1}\right\|^{2}\left\|x_{3}\right\|^{2}+\left\|x_{1}\right\|^{2}\left\|x_{2}\right\|^{2}-\left\langle x_{1}, x_{2}\right\rangle^{2}-\left\langle x_{1}, x_{3}\right\rangle^{2}-\left\langle x_{2}, x_{3}\right\rangle^{2}\right) .
\end{aligned}
$$

\subsection{Homotopy moment maps and equivariant cohomology}

In this section, we study the relationship between equivariant cohomology and homotopy moment maps. After interpreting $F^{\Omega}$ in terms of the Bott-Shulman-Stasheff complex, we provide general constructions of homotopy moment maps from cocycles in the BottShulman-Stasheff model (Proposition 3.3.10) as well as from cocycles in the Cartan model (Proposition 3.3.25, generalizing Theorem 3.2.1). The moment map for cocycles in the Cartan model arises via a chain map from the Cartan model to the Bott-Shulman-Stasheff model, which was outlined in [Mei05, App. C]. As we need to compute (a component) of the image of this map, we give a detailed description of this chain map. This section grew out of discussions with C. Rogers and M. Zambon and also appears in [CFRZ15].

\subsubsection{Differential forms on simplicial manifolds}

If $X_{\bullet}$ is a simplicial manifold with face maps $d_{i}: X_{n} \rightarrow X_{n-1}, i=0, \ldots, n$, then the simplicial differential $\partial_{n}: \Omega^{*}\left(X_{n}\right) \rightarrow \Omega^{*}\left(X_{n+1}\right)$ is

$$
\partial_{n}=\sum_{i=0}^{n+1}(-1)^{i} d_{i}^{*} .
$$


Consider the following double complex of differential forms on a simplicial manifold and its total complex

$$
\begin{aligned}
\Omega^{j, k}\left(X_{\bullet}\right) & :=\Omega^{k}\left(X_{j}\right), \\
\Omega^{*}\left(X_{\bullet}\right) & :=\left(\operatorname{Tot}\left(\Omega^{*, *}\left(X_{\bullet}\right)\right), \mathbf{d}\right), \\
\mathbf{d} & :=\partial+(-1)^{j} d,
\end{aligned}
$$

where $d$ is the exterior derivative.

If $X_{\bullet}$ is a simplicial manifold which is paracompact in each dimension, then the de Rham theorem of Bott-Shulman-Stasheff ([BSS76]) implies that there exists a natural isomorphism

$$
H\left(\Omega^{*}\left(X_{\bullet}\right)\right) \stackrel{\cong}{\rightarrow} H(\|X \bullet\|),
$$

where $H\left(\left\|X_{\bullet}\right\|\right)$ is the singular cohomology with $\mathbb{R}$ coefficients of the fat geometric realization of $X_{\text {. }}$.

3.3.1 Example. Let $M$ be a manifold and $M_{\text {}}$ the simplicial manifold $M_{n}=M$, whose face and degeneracy maps are $\operatorname{id}_{M}$. Since all $\partial_{n}$ are either zero or isomorphisms, the inclusion

$$
\left(\Omega^{n}(M), d\right)=\left(\Omega^{n}\left(M_{0}\right), d\right) \stackrel{\iota}{\hookrightarrow}\left(\Omega^{*}\left(M_{\bullet}\right), \mathbf{d}\right)
$$

is an quasi-isomorphism.

3.3.2 Example. Let $M$ be a $G$-manifold, and let $E_{\bullet} G \times M$ denote the product $E_{\bullet} G \times M_{\bullet}$, i.e., the simplicial manifold

$$
[n] \mapsto E_{n} G \times M=G^{n+1} \times M
$$

with the "usual" face and degeneracy maps, i.e.

$$
d_{i}\left(g_{0}, \ldots, g_{n}, p\right)=\left(g_{0}, \ldots, g_{i-1}, g_{i+1}, \ldots, g_{n}, p\right)
$$

If we equip $E_{\bullet} G \times M$ with the diagonal $G$ action

$$
\begin{aligned}
G \times E_{n} G \times M & \rightarrow M, \\
\left(h, g_{0}, \ldots, g_{n}, p\right) & \mapsto\left(g_{0} h^{-1}, \ldots, g_{n} h^{-1}, h p\right),
\end{aligned}
$$

then the projection $\pi: E_{\bullet} G \times M \rightarrow M_{\bullet}$ is a morphism of simplicial $G$-manifolds.

The idea for the following proof was pointed out to the author by C. Rogers:

3.3.3 Proposition. The map $\pi$ induces a quasi-isomorphism

$$
\pi^{*}: \Omega^{*}\left(M_{\bullet}\right) \rightarrow \Omega^{*}\left(E_{\bullet} G \times M\right) .
$$


Proof. Denote the (thin) geometric realization of $X_{\bullet}$ by $\left|X_{\bullet}\right|$. Since $|\cdot|$ preserves products, and since both $G$ and $M$ are manifolds, it follows from [Seg74, Prop. A1] and the de Rham theorem of Bott-Shulman-Stasheff ([BSS76]) that we have a commuting diagram

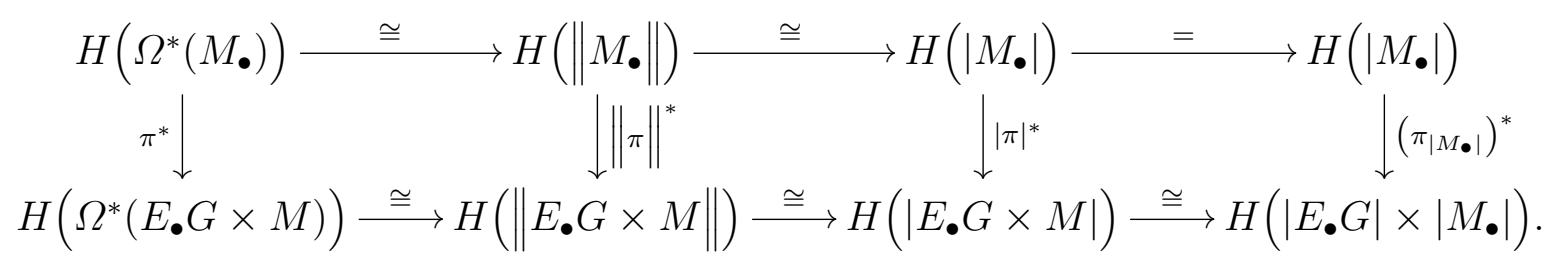

Since $\left|E_{\bullet} G\right|$ is contractible, the Künneth formula implies that the right vertical arrow in the diagram (3.12) is an isomorphism. Hence $\pi^{*}$ is also an isomorphism.

3.3.4 Example. (cf. [Mei05, App. C.2]) If $M$ is a $G$-manifold, consider the simplicial manifold $G^{\bullet} \times M$, i.e.

$$
[n] \mapsto G^{n} \times M
$$

with the face maps $d_{i}: G^{n} \times M \rightarrow G^{n-1} \times M$ given by

$$
\left(g_{1}, \ldots, g_{n}, p\right) \mapsto \begin{cases}\left(g_{2}, \ldots, g_{n}, p\right) & i=0, \\ \left(g_{1}, \ldots, g_{i} g_{i+1}, \ldots, g_{n}, p\right) & 0<i<n, \\ \left(g_{1}, \ldots, g_{n-1}, g_{n} p\right) & i=n .\end{cases}
$$

Note that the map

$$
\begin{aligned}
G^{n+1} \times M & \rightarrow G^{n} \times M, \\
\left(g_{0}, \ldots, g_{n}, p\right) & \mapsto\left(g_{0} g_{1}^{-1}, \ldots g_{n-1} g_{n}^{-1}, g_{n} p\right)
\end{aligned}
$$

induces an isomorphism of simplicial manifolds

$$
E_{\bullet} G \times_{G} M \cong G^{\bullet} \times M,
$$

where $E_{\bullet} \times{ }_{G} M$ is the quotient of $E_{\bullet} G \times M$ by the diagonal $G$-action (3.10). The de Rham theorem of Bott-Shulman-Stasheff $([\mathrm{BSS} 76])$ implies that the cohomology of $\left(\Omega^{*}\left(G^{\bullet} \times\right.\right.$ $M), \mathbf{d})$ is the equivariant cohomology of $M$. Therefore, the complex $\left(\Omega^{*}\left(G^{\bullet} \times M\right), \mathbf{d}\right)$ is called Bott-Shulman-Stasheff model for equivariant cohomology.

\subsubsection{Homotopy moment maps and the Bott-Shulman-Stasheff complex}

Consider the first row $\Omega^{1, *}\left(G^{\bullet} \times M\right)=\Omega^{*}(G \times M)$ of the Bott-Shulman-Stasheff complex and the subcomplex

$$
\Omega^{*}(G \times M)^{G} \subset \Omega^{1, *}\left(G^{\bullet} \times M\right)
$$

of forms invariant under the $G$-action $G \curvearrowright G \times M,(h,(g, p)) \mapsto(h g, p)$. This is the total complex of the double complex of $G$-invariant forms

$$
\begin{aligned}
\Omega^{k, m}(G \times M)^{G} & :=\Gamma\left(G \times M, \bigwedge^{k} T^{*} G \otimes \bigwedge^{m} T^{*} M\right)^{G} \subset \Omega^{k+m}(G \times M)^{G}, \\
\Omega^{*}(G \times M)^{G} & =\operatorname{Tot}\left(\Omega^{*, *}(G \times M)^{G}\right), \\
d & =d^{G}+(-1)^{k} d^{M}
\end{aligned}
$$


with differentials $d^{G}$ and $d^{M}$, the exterior derivatives in the $G$ and $M$ directions, respectively.

Consider the natural isomorphism

$$
\begin{gathered}
\Psi: \bigwedge^{m}\left(\mathfrak{g}^{\vee} \oplus T^{*} M\right) \stackrel{\cong}{\rightarrow} \bigoplus_{k+\ell=m} \bigwedge^{k}\left(\mathfrak{g}^{\vee}\right) \otimes \bigwedge^{\ell} T^{*} M, \\
\Psi(\alpha)\left(\left(x_{1}, \ldots, x_{k}\right) \otimes\left(w_{1}, \ldots, w_{\ell}\right)\right)=\alpha\left(\left(x_{1}, 0\right), \ldots,\left(x_{k}, 0\right),\left(0, w_{1}\right), \ldots,\left(0, w_{\ell}\right)\right) .
\end{gathered}
$$

3.3.5 Remark. The following diagram outlines the rest of this section and shows how (parts of) these various complexes are related and how the condition for $\tilde{f}$ to be a homotopy moment map can be understood in terms of the Bott-Shulman-Stasheff complex:

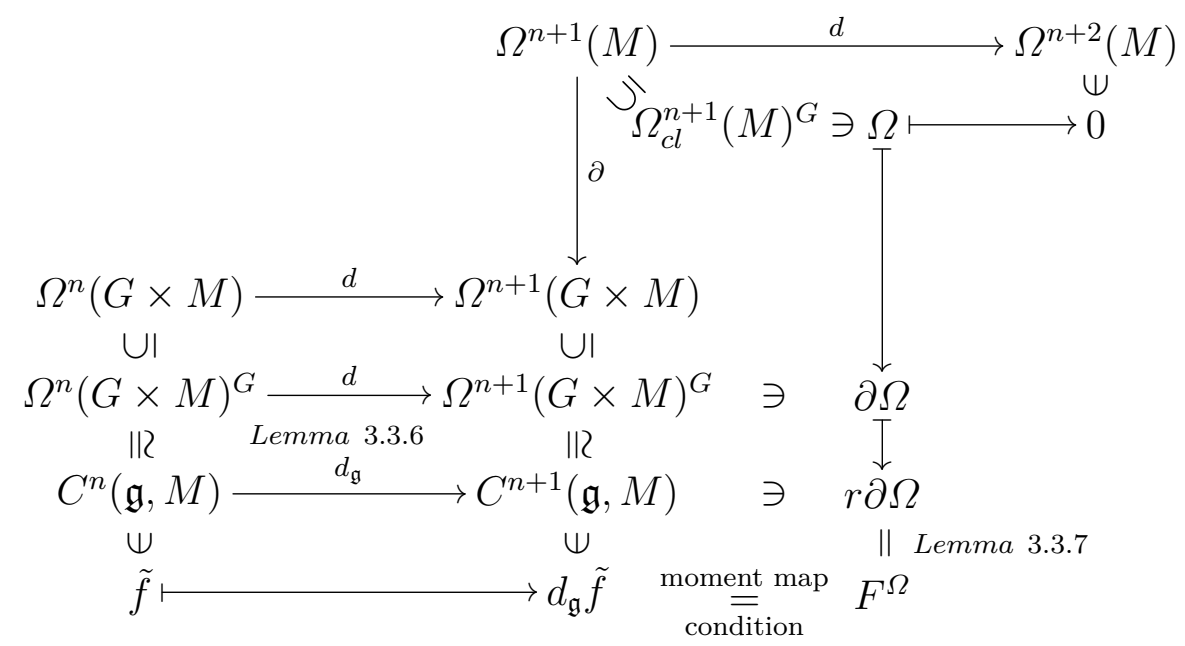

The consequence of this will be Proposition 3.3.10, in which we show that certain elements in the Bott-Shulman-Stasheff complex give rise to homotopy moment maps.

3.3.6 Lemma. Restriction to $M=\{e\} \times M \stackrel{i}{\hookrightarrow} G \times M$ induces an isomorphism of double complexes

$$
r:\left(\Omega^{*, *}(G \times M)^{G}, d^{G}, d^{M}\right) \rightarrow\left(C^{*, *}(\mathfrak{g}, M), \delta_{\mathfrak{g}}, d\right),
$$

where $G \curvearrowright G \times M,(h,(g, p)) \mapsto(h g, p)$. In particular, we have an isomorphism of total complexes:

$$
r:\left(\Omega^{*}(G \times M)^{G}, d\right) \rightarrow\left(C^{*}(\mathfrak{g}, M), d_{\mathfrak{g}}\right) .
$$

Proof. The restriction of sections of $\wedge^{m} T^{*}(G \times M)$ to $M=\{e\} \times M \hookrightarrow G \times M$ induces an isomorphism:

$$
\Gamma\left(G \times M, \bigwedge^{m}\left(T^{*}(G \times M)\right)\right)^{G} \rightarrow \Gamma\left(M, i^{*} \bigwedge^{m} T^{*}(G \times M)\right)=\Gamma\left(M, \bigwedge^{m}\left(\mathfrak{g}^{\vee} \oplus T^{*} M\right)\right) .
$$

Composing with $\Psi$ (3.16), we obtain the isomorphism $r: \Omega^{m}(G \times M)^{G} \rightarrow C^{m}(\mathfrak{g}, M)$. Finally, $r d^{M}=d r$ follows immediately from the definition of the differentials $d^{M}$ and $d . \square$

Now that we identified $C^{*}(\mathfrak{g}, M)$ as sitting inside $\Omega^{1, *}\left(G^{\bullet} \times M\right)$, we can reinterpret the term on the right hand side of the moment map condition (3.1) in terms of the Bott-Shulman-Stasheff complex. 
3.3.7 Lemma. Let $\Omega \in \Omega^{n+1}(M)^{G}$. Then

$$
r(\partial \Omega)=F^{\Omega} .
$$

Proof. The face map $d_{1}: G \times M \rightarrow M$ is the $G$-action. Therefore, it is $G$-equivariant and hence $d_{1}^{*} \Omega \in \Omega^{n+1}(G \times M)^{G}$. Since $d_{0}=\pi_{M}$, we also have $d_{0}^{*} \Omega=\pi_{M}^{*} \Omega \in \Omega^{n+1}(G \times M)^{G}$, and hence

$$
\partial \Omega=d_{0}^{*} \Omega-d_{1}^{*} \Omega \in \Omega^{n+1}(G \times M)^{G} .
$$

The differential of $d_{1}$ at the point $(e, p)$ is given by

$$
\left.d\left(d_{1}\right)\right|_{(e, p)}(x, w)=w-v_{x}^{G}, \text { for } x \in T_{e} G, w \in T_{p} M
$$

Let $x_{1}, \ldots, x_{n+1} \in \mathfrak{g}$ and $w_{1}, \ldots, w_{n+1} \in T_{p} M$. Then

$$
\begin{aligned}
r\left(d_{0}^{*} \Omega-d_{1}^{*} \Omega\right)\left(w_{1}, \ldots, w_{n+1}\right) & =0 \\
r\left(d_{0}^{*} \Omega-d_{1}^{*} \Omega\right)\left(x_{1}, \ldots, x_{k}, w_{1} \ldots, w_{n-k+1}\right) & =(-1)^{k+1}\left\langle\iota_{\mathfrak{g}}^{k} \Omega, x_{1}, \ldots, x_{k}\right\rangle\left(w_{1}, \ldots, w_{n-k+1}\right) .
\end{aligned}
$$

Thus $r \partial \Omega=\sum_{k=1}^{n+1}(-1)^{k+1} \iota_{\mathfrak{g}}^{k} \Omega=F^{\Omega}$.

3.3.8 Corollary. An element $f=\sum_{k=1}^{n} f_{k} \in C^{n}(\mathfrak{g}, M)$ with $f_{k} \in C^{k, n-k}(\mathfrak{g}, M)$ is a homotopy moment map for the pre-n-plectic form $\Omega \in \Omega^{n+1}(M)^{G}$ if and only if $\tilde{f}=$ $\sum_{k=1}^{n} \zeta(k) f_{k}$ satisfies $d_{\mathfrak{g}} \tilde{f}=r(\partial \Omega)$.

3.3.9 Remark. Note that the $\Omega^{0, n+1}(G \times M)^{G}$-component of $\partial \Omega$ vanishes. This is the reason why $f$ only has $n$ components $f_{k} \in \Lambda^{k}\left(\mathfrak{g}^{\vee}\right) \otimes \Omega^{n-k}(M), k=1, \ldots, n$. For a general solution $\eta \in \Omega^{n}(G \times M)^{G}$ of $d \eta=\partial \Omega$, this component does not vanish, but is an arbitrary closed $n$-form on $M$.

\subsubsection{Homotopy moment maps and Bott-Shulman-Stasheff cocycles}

If the group $G$ is compact, we can average to obtain $G$-invariant forms. For $\beta \in \Omega^{n+1}(M)$ and $\beta^{\prime} \in \Omega^{n}(M)$, denote $\beta^{G} \in \Omega^{n+1}(M)^{G}$ and $\beta^{\prime G} \in \Omega^{n}(G \times M)^{G}$ the $G$-invariant forms obtained by averaging with respect to the actions $G \curvearrowright M$ and $G \curvearrowright G \times M$, $(h,(g, p)) \mapsto(h g, p)$, respectively.

For $\alpha_{1} \in \Omega^{n}(G \times M)$, denote the component of $r\left(\alpha_{1}\right)$ in $C^{k, n-k}(\mathfrak{g}, M)$ by $r_{k}\left(\alpha_{1}\right)$, i.e. $r\left(\alpha_{1}\right)=\sum_{k=0}^{n} r_{k}\left(\alpha_{1}\right)$. Using the projection

$$
\begin{aligned}
& \check{r}: \Omega^{n}(G \times M)^{G} \stackrel{r}{\rightarrow} C^{n}(\mathfrak{g}, M) \rightarrow \bigoplus_{k=1}^{n} C^{k, n-k}(\mathfrak{g}, M), \\
& \check{r}\left(\alpha_{1}\right):=\sum_{k=1}^{n} r_{k}\left(\alpha_{1}\right) .
\end{aligned}
$$

Corollary 3.3.8 gives us two simple ways of constructing homotopy moment maps from cocycles in the Bott-Shulman-Stasheff complex: 
3.3.10 Proposition. Let $M$ be a $G$-manifold and $\alpha=\sum_{i=0}^{n+1} \alpha_{i} \in \Omega^{n+1}\left(G^{\bullet} \times M\right)$ a cocycle in the Bott-Shulman-Stasheff complex with $\alpha_{i} \in \Omega^{i, n-i+1}\left(G^{\bullet} \times M\right)=\Omega^{n-i+1}\left(G^{i} \times M\right)$.

- If $\alpha_{0} \in \Omega^{n+1}(M)^{G} \subset \Omega^{0, n+1}\left(G^{\bullet} \times M\right)$ and $\alpha_{1} \in \Omega^{n}(G \times M)^{G} \subset \Omega^{1, n}(G \bullet M)$, then $\tilde{f}:=\check{r} \alpha_{1}$ defines a homotopy moment map for the $G$-invariant pre-n-plectic form $\alpha_{0}$.

- Let $G$ be compact. Then $\tilde{f}:=\check{r}\left(\alpha_{1}^{G}\right)$ defines a homotopy moment map $f$ for the $G$-invariant pre-n-plectic form $\alpha_{0}^{G} \in \Omega^{n+1}(M)^{G}$.

Proof. The cocyle condition $\mathbf{d} \alpha=0$ implies that

$$
\begin{aligned}
& d \alpha_{0}=0, \\
& \partial \alpha_{0}=d \alpha_{1} .
\end{aligned}
$$

Therefore, $\alpha_{0}$ is indeed a pre- $n$-plectic form. The first claim follows immediately from Corollary 3.3.8 and the observation that $d_{\mathfrak{g}} r\left(\alpha_{1}\right)=d_{\mathfrak{g}} \check{r}\left(\alpha_{1}\right)$. For the second claim, we check that

$$
d_{\mathfrak{g}} r\left(\alpha_{1}^{G}\right)=r\left(d \alpha_{1}^{G}\right)=r\left(\left(d \alpha_{1}\right)^{G}\right)=r\left(\left(\partial \alpha_{0}\right)^{G}\right)=r\left(\partial \alpha_{0}^{G}\right)
$$

and again observe that $d_{\mathfrak{g}} r\left(\alpha_{1}^{G}\right)=d_{\mathfrak{g}} \check{r}\left(\alpha_{1}^{G}\right)$.

3.3.11 Remark. Note that we do not need a full cocycle in the Bott-Shulman-Stasheff complex, but only a $G$-invariant 1-step extension of $\alpha_{0} \in \Omega^{n+1}(M)^{G}$ in the Bott-ShulmanStasheff complex, i.e. $\alpha_{1} \in \Omega^{n}(G \times M)^{G}$ satisfying $d \alpha_{0}=0$ and $\partial \alpha_{0}=d \alpha_{1}$ (cf. Remark 3.3.5).

This also recovers the homotopy moment map for exact pre- $n$-plectic forms constructed in [FRZ13, Lem. 8.1]:

3.3.12 Corollary. If $\Omega=d \beta \in \Omega^{n+1}(M)^{G}$ is an exact pre-n-plectic form with $\beta \in$ $\Omega^{n}(M)^{G}$, then

$$
f:=\sum_{k=1}^{n} \zeta(k+1) \iota_{\mathfrak{g}}^{k} \beta
$$

is a homotopy moment map.

Proof. Take $\alpha_{0}:=\Omega$ and $\alpha_{1}:=\partial \beta$. Then

$$
d \alpha_{1}=d \partial \beta=\partial d \beta=\partial \alpha_{0} .
$$

Using Lemma 3.3.7, we obtain the homotopy moment map $f$ from $\tilde{f}=r \partial \alpha_{1}=r \partial \beta$.

3.3.13 Corollary. Let $\alpha=\mathbf{d} \beta$ be a coboundary in the Bott-Shulman-Stasheff complex with $\beta=\sum_{i=0}^{n} \beta_{i} \in \Omega^{n}\left(G^{\bullet} \times M\right)$, where $\beta_{i} \in \Omega^{i, n-i}\left(G^{\bullet} \times M\right), \beta_{0} \in \Omega^{n}(M)^{G}$ and $\beta_{1} \in \Omega^{n-1}(G \times M)^{G}$. Then $\tilde{f}=\check{r} \alpha_{1}=\check{r}\left(\partial \beta_{0}-d \beta_{1}\right)$ defines a homotopy moment map $f$ for the pre-n-plectic form $\alpha_{0}=d \beta_{0}$, which is given by

$$
\tilde{f}=\sum_{k=1}^{n}(-1)^{k+1} \iota_{\mathfrak{g}}^{k} \beta_{0}-\delta_{\mathfrak{g}} \check{\gamma} \beta_{1}-\sum_{k=1}^{n-1}(-1)^{k} d r_{k}\left(\beta_{1}\right) .
$$


In particular, given two cocycles in the Bott-Shulman-Stasheff complex with G-invariant components in $\Omega^{0, n}\left(G^{\bullet} \times M\right)$ and $\Omega^{1, n-1}\left(G^{\bullet} \times M\right)$ and which differ by a coboundary $\mathbf{d} \beta$, the associated homotopy moment maps differ by $r \partial \beta_{0}-\delta_{\mathfrak{g}} \check{r} \beta_{1}-\sum_{k=1}^{n-1}(-1)^{k} d r_{k}\left(\beta_{1}\right)$.

Proof. From $\alpha=\mathbf{d} \beta$, we have $\alpha_{0}=d \beta_{0}$ and $\alpha_{1}=\partial \beta_{0}-d \beta_{1}$. Since $\beta_{0}$ and $\beta_{1}$ are $G$-invariant, it follows that $\alpha_{0}$ and $\alpha_{1}$ are as well. Therefore, we can apply the second part of Proposition 3.3.10. We obtain

$$
\begin{aligned}
\tilde{f} & =\check{r} \alpha_{1}=\check{r} \partial \beta_{0}-\check{r} d \beta_{1} \\
& =\sum_{k=1}^{n}(-1)^{k+1} \iota_{\mathfrak{g}}^{k} \beta_{0}-\sum_{k=1}^{n} r_{k}\left(\left(d^{G}+(-1)^{k} d^{M}\right) \beta_{1}\right) \\
& =\sum_{k=1}^{n}(-1)^{k+1} \iota_{\mathfrak{g}}^{k} \beta_{0}-\sum_{k=1}^{n-1}\left(\delta_{\mathfrak{g}}+(-1)^{k} d\right) r_{k}\left(\beta_{1}\right) \\
& =\sum_{k=1}^{n}(-1)^{k+1} \iota_{\mathfrak{g}}^{k} \beta_{0}-\delta_{\mathfrak{g}} \check{r}\left(\beta_{1}\right)-\sum_{k=1}^{n-1}(-1)^{k} d r_{k}\left(\beta_{1}\right) .
\end{aligned}
$$

3.3.14 Remark. Note that adding a coboundary $\mathrm{d} \beta$ to a cocycle $\alpha$ will change the pre$n$-plectic form $\alpha_{0}$ to $\alpha_{0}+d \beta_{0}$. However, the construction of the tuple of the pre- $n$-plectic form and the homotopy moment map from a cocylce is linear.

\subsubsection{Simplicial differential forms}

We recall the notion of simplicial differential forms introduced by Dupont [Dup76, Def. 2.1]:

Let $X$. be a simplicial manifold with face maps $d_{i}: X_{q} \rightarrow X_{q-1}$ for $i=0, \ldots, q$. Let $\Delta^{q} \subset \mathbb{R}^{q+1}$ be the standard $q$-simplex and $\varepsilon_{i}: \Delta^{q-1} \rightarrow \Delta^{q}$ the inclusion of the $i$-th face.

A simplicial differential $n$-forms $\varphi$ on $X$. consists of a sequence of forms

$$
\varphi^{(q)} \in \Omega^{n}\left(\Delta^{q} \times X_{q}\right), q=0,1, \ldots
$$

satisfying

$$
\left(\varepsilon_{i} \times \mathrm{id}\right)^{*} \varphi^{(q)}=\left(\mathrm{id} \times d_{i}\right)^{*} \varphi^{(q-1)}
$$

for all $q$ and all $i=1, \ldots, q$.

The set of all simplicial $n$-forms on $X_{\bullet}$ is denoted $\Omega_{s p l}^{n}\left(X_{\bullet}\right)$. Equipped with the usual exterior derivative $d$, simplicial differential forms form a differential graded algebra $\left(\Omega_{s p l}^{*}\left(X_{\bullet}\right), d\right)$, which is also the total complex of the following double complex:

$$
\Omega_{s p l}^{n}\left(X_{\bullet}\right)=\bigoplus_{j+k=n} \Omega_{s p l}^{j, k}\left(X_{\bullet}\right)
$$

Here, similar to $(3.14), \Omega_{s p l}^{j, k}\left(X_{\bullet}\right)$ consists of simplicial differential $n$-forms $\varphi=\left(\varphi^{(q)}\right)$, for which each

$$
\varphi^{(q)} \in \Gamma\left(\Delta^{q} \times X_{q}, \bigwedge^{j} T^{*} \Delta^{q} \otimes \bigwedge^{k} T^{*} X_{q}\right) \subset \Omega^{j+k}\left(\Delta^{q} \times X_{q}\right) .
$$


The exterior derivative $d$ on $\Omega_{s p l}^{*}\left(X_{\bullet}\right)$ is

$$
d=d^{\Delta}+(-1)^{j} d^{X}
$$

where $d^{\Delta}$ and $d^{X}$ denote the exterior derivatives in the $\Delta^{q}$ and $X_{q^{-}}$-directions, respectively.

3.3.15 Remark. Intuitively, simplicial $n$-forms should be thought of as $n$-forms on the fat geometric realization $\left\|X_{\bullet}\right\|$ of $X_{\bullet}$.

Dupont proved that $\Omega^{*}\left(X_{\bullet}\right)$ and $\Omega_{s p l}^{*}\left(X_{\bullet}\right)$ are quasi-isomorphic.

3.3.16 Theorem ([Dup76, Thm. 2.3]). There are natural maps of doubles complexes

$$
\left(\Omega_{\text {spl }}^{*, *}\left(X_{\bullet}\right), d^{\Delta}, d^{X}\right) \underset{\mathscr{C}}{\stackrel{\mathscr{I}}{\rightleftarrows}}\left(\Omega^{*, *}\left(X_{\bullet}\right), \partial, d\right),
$$

which give natural chain homotopy equivalences between $\left(\Omega_{s p l}^{*, k}\left(X_{\bullet}\right), d^{\Delta}\right)$ and $\left(\Omega^{*, k}\left(X_{\bullet}\right), \partial\right)$. In particular, the maps $\mathscr{C}$ and $\mathscr{I}$ induce quasi-isomorphisms between the total complexes $\left(\Omega_{\text {spl }}^{*}\left(X_{\bullet}\right), d\right)$ and $\left(\Omega^{*}\left(X_{\bullet}\right), \mathbf{d}\right)$.

The map $\mathscr{I}$ in Dupont's theorem is defined as the fibre integral

$$
\Omega_{s p l}^{j, k}\left(X_{\bullet}\right) \ni \varphi \mapsto \mathscr{I}(\varphi):=\int_{\Delta^{j}} \varphi^{(j)} \in \Omega^{k}\left(X_{j}\right) .
$$

The map $\mathscr{C}$ is defined as follows:

$$
\mathscr{C}(\beta)^{(q)}:= \begin{cases}j ! \sum_{|I|=j} \sum_{\ell=0}^{j}(-1)^{\ell} t_{i_{\ell}} d t_{i_{0}} \wedge \ldots \wedge \widehat{d t_{i_{\ell}}} \wedge \ldots \wedge d t_{i_{j}} \wedge \mu_{I}^{*} \beta & q \geq j \\ 0 & q<j,\end{cases}
$$

for $\beta \in \Omega^{k}\left(X_{j}\right)$, where $I=\left(i_{0}, \ldots, i_{j}\right)$ is a multi-index with $0 \leq i_{0}<\cdots<i_{j} \leq q$ and $|I|:=j$. Furthermore, $\mu_{I}=d_{\tilde{\iota}_{q-j}} \circ \ldots \circ d_{\tilde{\iota}_{1}}: X_{q} \rightarrow X_{j}$ is the face map corresponding to the complementary sequence $0 \leq \tilde{\iota}_{1}<\cdots<\tilde{\iota}_{q-j} \leq q$ of $I$.

\subsubsection{Cartan complexes}

If $A$ is a $G^{\star}$-module in the sense of Definition 2.2.51 (also cf. [GS99, Def. 2.3.1]), with differential $d^{A}$ and insertion operation $\iota_{\mathfrak{g}}^{A}$, let

$$
\begin{aligned}
C_{G}(A): & =\left(S\left(\mathfrak{g}^{\vee}\right) \otimes A\right)^{G} \\
d_{G} & =\delta+d^{A}
\end{aligned}
$$

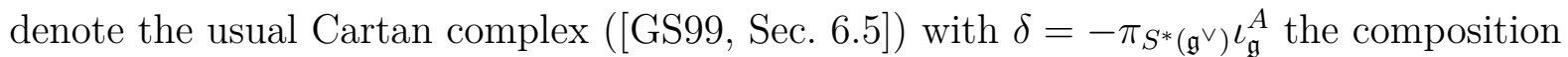
of $-\iota_{\mathfrak{g}}^{A}$ and the symmetrization projection $\pi_{S^{*}\left(\mathfrak{g}^{\vee}\right)}: \mathfrak{g}^{\vee} \otimes S^{*}\left(\mathfrak{g}^{\vee}\right) \rightarrow S^{*+1}\left(\mathfrak{g}^{\vee}\right)$. This is also the total complex of the double complex

$$
C_{G}^{i, j}(A)=\left(S^{i}\left(\mathfrak{g}^{\vee}\right) \otimes A^{j-i}\right)^{G} .
$$

The following Lemma provides a criterion for a chain map $\phi: A \rightarrow B$ to induce a quasiisomorphism $C_{G}(A) \rightarrow C_{G}(B)$. The author is grateful to C. Rogers for pointing out [McC01, Thm. 3.5]. 
3.3.17 Lemma. Let $G$ be a compact Lie group, $A$ and $B$ two $G^{\star}$-modules which are bounded below as complexes and let $\phi: A \rightarrow B$ a quasi-isomorphism of $G^{\star}$-modules, i.e., a morphism of $G^{\star}$-modules, which induces an isomorphism of $G$-modules on total cohomology. Then the induced map of Cartan complexes

$$
\operatorname{id}_{S^{*}\left(\mathfrak{g}^{\vee}\right)} \otimes \phi: C_{G}(A) \rightarrow C_{G}(B)
$$

is a quasi-isomorphism.

Proof. For a $G^{\star}$-module $A$, define the decreasing filtration on $C_{G}(A)$ :

$$
F_{p} C_{G}(A):=\bigoplus_{i \geq p} \bigoplus_{j} C_{G}^{i, j}(A)
$$

If $A$ is bounded below, then the associated spectral sequence clearly converges.

The induced map $\operatorname{id}_{S^{*}\left(\mathfrak{g}^{\vee}\right)} \otimes \phi$ respects the filtrations associated to $A$ and $B$. Since $\phi$ is a quasi-isomorphism and $G$ is compact, $\mathrm{id}_{S\left(\mathfrak{g}^{*}\right)} \otimes \phi$ induces an isomorphism between the $E_{1}$ pages

$$
E_{1}^{p, q}(A)=\left(S^{p}\left(\mathfrak{g}^{\vee}\right) \otimes H^{q-p}(A)\right)^{G} \rightarrow\left(S^{p}\left(\mathfrak{g}^{\vee}\right) \otimes H^{q-p}(B)\right)^{G}=E_{1}^{p, q}(B)
$$

of the associated spectral sequences (e.g. [GS99, Thm. 6.5.1]). Since $A$ and $B$ are bounded below, the filtrations are bounded in each degree. Therefore, $\operatorname{id}_{S\left(\mathfrak{g}^{*}\right)} \otimes \pi^{*}$ is a quasi-isomorphism (e.g. [McC01, Thm. 3.5]).

3.3.18 Example. For $M$ a $G$-manifold the Cartan complex of $\Omega^{*}(M)$ with the usual $G^{\star}$-module structure is the usual Cartan complex for $M$ :

$$
C_{G}(M):=C_{G}\left(\Omega^{*}(M)\right) \text {. }
$$

3.3.19 Example. For a simplicial $G$-manifold $X_{\bullet}$, the total complex of differential forms $\Omega^{*}\left(X_{\bullet}\right)=\operatorname{Tot}\left(\Omega^{* * *}\left(X_{\bullet}\right)\right)$ with differential $\mathbf{d}=\partial+(-1)^{j} d$ and the insertion operation $\iota_{\mathfrak{g}}^{\Omega^{*}\left(X_{\bullet}\right)}:=(-1)^{j} \iota_{\mathfrak{g}}$ is a $G^{\star}$-module. Note that

$$
\mathbf{d} \iota_{\mathfrak{g}}^{\Omega^{*}\left(X_{\bullet}\right)}+\iota_{\mathfrak{g}}^{\Omega^{*}\left(X_{\bullet}\right)} \mathbf{d}=d \iota_{\mathfrak{g}}+\iota_{\mathfrak{g}} d
$$

is still the usual Lie derivative. Its Cartan complex

$$
\begin{aligned}
C_{G}^{*}\left(X_{\bullet}\right) & :=\left(C_{G}\left(\operatorname{Tot}\left(\Omega^{*, *}\left(X_{\bullet}\right)\right), \mathbf{d}_{G}\right),\right. \\
\mathbf{d}_{G} & :=(-1)^{j} \delta+\mathbf{d}=\partial+(-1)^{j} \delta+(-1)^{j} d .
\end{aligned}
$$

The Cartan complex $C_{G}\left(X_{\bullet}\right)$ is also the total complex of the tricomplex

$$
C_{G}^{i, j, k}\left(X_{\bullet}\right):=\left(S^{i}\left(\mathfrak{g}^{\vee}\right) \otimes \Omega^{k-i}\left(X_{j}\right)\right)^{G} .
$$


3.3.20 Example. For a simplicial $G$-manifold $X_{\bullet}$, consider the Cartan complex of $\Omega_{s p l}^{*}\left(X_{\bullet}\right)$ with its usual $G^{*}$-module structure:

$$
\begin{aligned}
& C_{G, s p l}^{*}\left(X_{\bullet}\right):=C_{G}\left(\Omega_{s p l}^{*}\left(X_{\bullet}\right)\right), \\
& \mathbf{d}_{G}=\delta+d .
\end{aligned}
$$

Also note that this is the total complex of the tricomplex

$$
\begin{aligned}
C_{G, s p l}^{i, j, k}\left(X_{\bullet}\right) & :=\left(S^{i}\left(\mathfrak{g}^{\vee}\right) \otimes \Omega_{s p l}^{j, k-i}\left(X_{\bullet}\right)\right)^{G} \subset \prod_{q=0}^{\infty}\left(S^{i}\left(\mathfrak{g}^{\vee}\right) \otimes \Omega^{j, k-i}\left(\Delta^{q} \times X_{q}\right)\right)^{G}, \\
C_{G, s p l}^{*}\left(X_{\bullet}\right) & :=\operatorname{Tot}\left(C_{G, s p l}^{*, * *}\left(X_{\bullet}\right)\right), \\
\mathbf{d}_{G} & :=\delta+d^{\Delta}+(-1)^{j} d^{X} .
\end{aligned}
$$

The $G^{\star}$-module structures on $\Omega(M), \Omega^{*}\left(E_{\bullet} G \times M\right)$ and $\Omega_{s p l}^{*}\left(E_{\bullet} G \times M\right)$ are chosen in such a way, that the quasi-isomorphisms $\iota(3.8), \pi^{*}(3.11)$ and Dupont's map $\mathscr{C}$ (3.21) are maps of $G^{\star}$-module. Therefore, Lemma 3.3.17 now implies

3.3.21 Proposition. If $G$ is compact, then the map induced by $\mathscr{C} \circ \pi^{*} \circ \iota: \Omega^{*}(M) \rightarrow$ $\Omega_{\text {spl }}^{*}\left(E_{\bullet} G \times M\right)$ on the total Cartan complexes

$$
\jmath:=\operatorname{id}_{S\left(\mathfrak{g}^{\vee}\right)} \otimes\left(\mathscr{C} \circ \pi^{*} \circ \iota\right): C_{G}^{*}(M) \rightarrow C_{G, s p l}^{*}\left(E_{\bullet} G \times M\right) .
$$

is a quasi-isomorphism.

\subsubsection{The Cartan map}

Let $\pi: P \rightarrow B$ be a principal $G$-bundle. Cartan [Car51] constructed a chain map $C_{G}^{*}(P) \rightarrow \Omega^{*}(B)$ that is knows as the Cartan map:

Pick a connection $A \in \Omega^{1}(P, \mathfrak{g})^{G}$ on $P \rightarrow B$. Denote its curvature by $F_{A}=d A+\frac{1}{2}[A, A] \in$ $\Omega^{2}(P, \mathfrak{g})_{h o r}^{G}$ and let hor $A: \Omega^{*}(P)^{G} \rightarrow \Omega^{*}(P)_{h o r}^{G}$ be the projection to horizontal forms defined by $A$. Then

$$
\begin{gathered}
\operatorname{Car}^{A}: C_{G}^{*}(P) \rightarrow \Omega^{*}(P)_{h o r}^{G} \cong \Omega^{*}(B), \\
\left(S^{i}\left(\mathfrak{g}^{\vee}\right) \otimes \Omega^{*}(P)\right)^{G} \ni \beta \mapsto \operatorname{hor}_{A}\left(\left\langle F_{A}^{i}, \beta\right\rangle\right) \in \Omega^{*+2 i}(P)_{h o r}^{G} .
\end{gathered}
$$

3.3.22 Remark. Recall that $\pi^{*}: \Omega^{*}(B) \rightarrow \Omega^{*}(P)_{h o r}^{G}$ is an isomorphism. If $G$ is compact, then the inclusion

$$
\Omega^{*}(B) \stackrel{\pi^{*}}{\longrightarrow} \Omega^{*}(P)_{h o r}^{G} \hookrightarrow C_{G}^{*}(P)
$$

induces an isomorphism in cohomology $H^{*}(B) \rightarrow H_{G}^{*}(P)$, with homotopy inverse $\mathrm{Car}^{A}$.

There is also a simplicial version of this construction: Let $P_{\bullet} \rightarrow B_{\bullet}$ be a simplicial principal $G$-bundle with a simplicial connection $A \in \Omega_{s p l}^{1}\left(P_{\bullet}, \mathfrak{g}\right)^{G}$. The connection $A$ is defined by a sequence of 1 -forms $A^{(q)} \in \Omega^{1}\left(\Delta^{q} \times P_{q}, \mathfrak{g}\right)^{G}$, where each $A^{(q)}$ is a connection 
on the principal $G$-bundle $\Delta^{q} \times P_{q} \rightarrow \Delta^{q} \times B_{p}$. Applying the degree-wise Cartan maps $\operatorname{Car}^{A^{(q)}}: C_{G}^{*}\left(\Delta^{q} \times P_{q}\right) \rightarrow \Omega^{*}\left(\Delta^{q} \times B_{q}\right)$ gives a chain map between the total complexes (3.31) and (3.19)

$$
\operatorname{Car}^{A}: C_{G, s p l}^{*}\left(P_{\bullet}\right) \rightarrow \Omega_{s p l}^{*}\left(B_{\bullet}\right)
$$

If $G$ is compact, then $\operatorname{Car}^{A}$ is a quasi-isomorphism.

3.3.23 Example. Let $M$ be a $G$-manifold and $P_{\bullet}:=E_{\bullet} G \times M \rightarrow E_{\bullet} G \times_{G} M$ the simplicial principal $G$-bundle. For $i=0, \ldots, q$, let

$$
\pi_{i}: E_{q} G \times M=G^{q+1} \times M \rightarrow G
$$

denote the projections and let $\theta_{L} \in \Omega^{1}(G, \mathfrak{g})^{G}$ denote the left-invariant Maurer-Cartan form on $G$. Following Dupont [Dup76], we consider the distinguished simplical connection

$$
\begin{aligned}
\theta & =\left(\theta^{(q)}\right) \in \Omega_{\text {spl }}^{0,1}\left(E_{\bullet} G \times M, \mathfrak{g}\right)^{G}, \\
\theta^{(q)} & :=\sum_{i=0}^{q} t_{i} \pi_{i}^{*} \theta_{L} \in \Omega^{1}\left(\Delta^{q} \times E_{q} G \times M, \mathfrak{g}\right)^{G},
\end{aligned}
$$

where $t_{i}, i=0, \ldots, q$ are barycentric coordinates on $\Delta^{q}$. The curvature of $\theta$ is

$F_{\theta^{(q)}}=\underbrace{d^{\Delta} \theta^{(q)}}_{\text {type 1,1 }}+\underbrace{d^{E_{q} G \times M} \theta^{(q)}+\frac{1}{2}\left[\theta^{(q)}, \theta^{(q)}\right]}_{\text {type } 0,2} \in \Omega^{1,1}\left(\Delta^{q} \times E_{q} G \times M\right)_{h o r}^{G} \oplus \Omega^{0,2}\left(\Delta^{q} \times E_{q} G \times M\right)_{h o r}^{G}$.

For example, for $q=1$, we have

$$
\begin{aligned}
\theta^{(1)} & =t_{0} \pi_{0}^{*} \theta_{L}+t_{1} \pi_{1}^{*} \theta_{L}, \\
F_{\theta^{(1)}} & =-d t_{1} \wedge\left(\pi_{0}^{*} \theta_{L}-\pi_{1}^{*} \theta_{L}\right)-\frac{t_{0} t_{1}}{2}\left[\pi_{0}^{*} \theta_{L}-\pi_{1}^{*} \theta_{L}, \pi_{0}^{*} \theta_{L}-\pi_{1}^{*} \theta_{L}\right],
\end{aligned}
$$

3.3.24 Remark. Note that $\theta=\mathscr{C}\left(\pi_{0}^{*} \theta_{L}\right)$, with $\pi_{0}^{*} \theta_{L} \in \Omega^{1}\left(E_{0} G \times M, \mathfrak{g}\right)^{G}=\Omega^{1}(G \times$ $M, \mathfrak{g})^{G}$ the pullback of the left-invariant Maurer-Cartan form.

\subsubsection{Cartan complex and Bott-Shulman-Stasheff complex}

By composing the chaim maps defined above, we obtain the chain map constructed in [Mei05, App. C], which, if $G$ is compact, is a quasi-isomorphism between the Cartan complex and the Bott-Shulman-Stasheff complex:

3.3.25 Proposition. Let $G$ be a Lie group and $M$ a $G$-manifold. Then there is a natural chain map from the Cartan model to the Bott-Shulman-Stasheff model

$$
C_{G}^{*}(M) \stackrel{\jmath}{\rightarrow} C_{G, s p l}^{*}\left(E_{\bullet} G \times M\right) \stackrel{\mathrm{Car}^{\theta}}{\longrightarrow} \Omega_{s p l}^{*}\left(G^{\bullet} \times M\right) \stackrel{\mathscr{I}}{\rightarrow} \Omega^{*}\left(G^{\bullet} \times M\right)
$$

where

- $\mathrm{j}$ is the chain map from Proposition 3.3.21, 
- $\operatorname{Car}^{\theta}$ is the simplicial Cartan map (3.34) for the simplical connection $\theta$ (3.36) on $E_{\bullet} G \times M \rightarrow E_{\bullet} G \times_{G} M \cong G^{\bullet} \times M$,

- I is the quasi-isomorphisms (3.20) defined by Dupont in Theorem 3.3.16.

If $G$ is compact, then all of the above are quasi-isomorphisms and hence $C_{G}^{*}(M) \rightarrow$ $\Omega^{*}\left(G^{\bullet} \times M\right)$ is a quasi-isomorphism.

3.3.26 Remark. Note that if $G$ is not compact, J, $\mathscr{C}$ and $\operatorname{Car}^{\theta}$ can fail to be quasiisomorphisms.

\subsubsection{Homotopy moment maps from Cartan cocycles}

We will now combine Proposition 3.3.25 and Proposition 3.3.10 to obtain an explicit homotopy moment map for each cocycle in the Cartan complex, generalizing [FRZ13, Thm. 6.3]:

3.3.27 Theorem. Given a degree $n+1$ Cartan cocycle $\Omega+\sum_{i=1}^{\left\lfloor\frac{n+1}{2}\right\rfloor} P_{i} \in C_{G}^{n+1}(M)$, with $\Omega \in \Omega^{n+1}(M)^{G}$ and $P_{i} \in\left(S^{i}\left(\mathfrak{g}^{\vee}\right) \otimes \Omega^{n-2 i+1}(M)\right)^{G}$, there is a natural homotopy moment map $f$ for the $G$-action on the pre-n-plectic manifold $(M, \Omega)$. More precisely, for $k=1, \ldots, n$ we have

$$
f_{k}=\sum_{i=1}^{\left\lfloor\frac{k+1}{2}\right\rfloor} \frac{(-1)^{i} \zeta(k) i !(k-i) !}{2^{i-1}(k-2 i+1) !} \pi_{\wedge^{k}\left(\mathfrak{g}^{\vee}\right)}\left(\iota_{\mathfrak{g}}^{k-2 i+1} P_{i}(\cdot \underbrace{[\cdot, \cdot], \ldots,[\cdot, \cdot]}_{i-1})\right),
$$

where $\pi_{\wedge^{k}\left(\mathfrak{g}^{\vee}\right)}$ is the skew-symmetrization projection and $\zeta(k)=-(-1)^{\frac{k(k+1)}{2}}$.

In particular, the homotopy moment map $f$ is G-equivariant, i.e., $f_{k} \in\left(\wedge^{k}\left(\mathfrak{g}^{\vee}\right) \otimes\right.$ $\left.\Omega^{n-k}(M)\right)^{G}$.

Proof. Given the chain map from Proposition 3.3.25 and the second part of Proposition 3.3.10, we immediately obtain a homotopy moment map from the cocycle $\Omega+$ $\sum_{i=1}^{\left\lfloor\frac{n+1}{2}\right\rfloor} P_{i} \in C_{G}^{n+1}(M)$ if the Lie group $G$ is compact. However, we will compute the $\Omega^{1, n}\left(G^{\bullet} \times M\right)=\Omega^{n}(G \times M)$ and $\Omega^{0, n+1}\left(G^{\bullet} \times M\right)=\Omega^{n+1}(M)$-components of the image of $\Omega+\sum_{i=1}^{\left\lfloor\frac{n+1}{2}\right\rfloor} P_{i}$ in $\Omega^{n+1}\left(G^{\bullet} \times M\right)$ and observe that these are $G$-invariant for $G$ an arbitrary (possibly non-compact) Lie group. This will then allow us to use the first part of Proposition 3.3.10 to construct a homotopy moment map.

First note, that the images of $\Omega$ and $P_{i}$ under $C_{G}(M) \rightarrow C_{G}^{*}\left(E_{\bullet} G \times M\right)$ are

$$
\begin{array}{r}
\pi_{M}^{*} \Omega \in \Omega^{n+1}(G \times M)^{G}=C_{G}^{0,0, n+1}\left(E_{\bullet} G \times M\right), \\
\pi_{M}^{*} P_{i} \in\left(S^{i}\left(\mathfrak{g}^{\vee}\right) \otimes \Omega^{n-2 i+1}(G \times M)\right)^{G}=C_{G}^{i, 0, n-i+1}\left(E_{\bullet} G \times M\right),
\end{array}
$$


respecively, where $\pi_{M}: G \times M \rightarrow M$ is the projection. Therefore, $\Omega+\sum_{i=1}^{\left\lfloor\frac{n+1}{2}\right\rfloor} P_{i}$ is mapped to

$$
\pi_{M}^{*} \Omega+\sum_{i=1}^{\left\lfloor\frac{n+1}{2}\right\rfloor} \pi_{M}^{*} P_{i} \in C_{G}^{n+1}\left(E_{\bullet} G \times M\right) .
$$

The elements

$$
\begin{aligned}
& \jmath(\Omega) \in C_{G, s p l}^{0,0, n+1}\left(E_{\bullet} G \times M\right) \subset \prod_{q=0}^{\infty} \Omega^{0, n+1}\left(\Delta^{q} \times E_{q} G \times M\right)^{G}, \\
& \jmath\left(P_{i}\right) \in C_{G, s p l}^{i, 0, n-i+1}\left(E_{\bullet} G \times M\right) \subset \prod_{q=0}^{\infty}\left(S^{i}\left(\mathfrak{g}^{\vee}\right) \otimes \Omega^{0, n-2 i+1}\left(\Delta^{q} \times E_{q} G \times M\right)\right)^{G},
\end{aligned}
$$

are given by the sequences $\jmath(\Omega)^{(q)}=\pi_{M}^{*} \Omega \in \Omega^{0, n+1}\left(\Delta^{q} \times E_{q} G \times M\right)^{G}$ and $\jmath\left(P_{i}\right)^{(q)}=\pi_{M}^{*} P_{i}$, respectively.

The next step is to compute the Cartan map of $\jmath(\Omega)$ and $\jmath\left(P_{i}\right)$, i.e. to compute $\operatorname{Car}^{\theta^{(q)}}\left(\pi_{M}^{*} \Omega\right)$ and $\operatorname{Car}^{\theta^{(q)}}\left(\pi_{M}^{*} P_{i}\right)$ for all $q$. Recall that the Cartan map was defined by inserting the curvature, taking a horizontal component and then pushing the resulting $G$-invariant horizontal form down to the base. Since the bundle $E_{q} G \times M \rightarrow E_{q} G \times_{G} M \cong$ $G^{q} \times M$ is trivial with section $s$ (cf. [Mei05, App. C.2])

$$
\begin{aligned}
s: G^{q} \times M & \rightarrow G^{q+1} \times M=E_{q} G \times M, \\
\left(g_{1}, \ldots, g_{q}, p\right) & \mapsto\left(e, g_{1}^{-1}, \ldots,\left(g_{1} \cdots g_{q}\right)^{-1}, g_{1} \cdots g_{q} p\right),
\end{aligned}
$$

we have

$$
\begin{aligned}
& \operatorname{Car}^{\theta^{(q)}}\left(\pi_{M}^{*} \Omega\right)=s^{*} \operatorname{hor}_{\theta^{(q)}} \pi_{M}^{*} \Omega, \\
& \operatorname{Car}^{\theta^{(q)}}\left(\pi_{M}^{*} P_{i}\right)=s^{*} \operatorname{hor}_{\theta^{(q)}}\left\langle F_{\theta^{(q)}}^{i}, \pi_{M}^{*} P_{i}\right\rangle=\left\langle s^{*} F_{\theta^{(q)}}^{i}, s^{*} \operatorname{hor}_{\theta^{(q)}} \pi_{M}^{*} P_{i}\right\rangle .
\end{aligned}
$$

Keeping in mind that Proposition 3.3.10 only uses the components in $\Omega^{0, n+1}\left(G^{\bullet} \times M\right)$ and $\Omega^{1, n}\left(G^{\bullet} \times M\right)$, we only need to compute the $(0, n+1)$ and $(1, n)$-components of $\mathscr{I}\left(\operatorname{Car}^{\theta}\left(\pi_{M}^{*} \Omega\right)\right)$ and $\mathscr{I}\left(\operatorname{Car}^{\theta}\left(\pi_{M}^{*} P_{i}\right)\right)$.

From the definition of $\mathscr{I}$, we see that the $\Omega^{0, n+1}\left(G^{\bullet} \times M\right)$-components are computed by applying $\int_{\Delta^{0}} \operatorname{Car}^{\theta^{(0)}}$ to $\pi_{M}^{*} \Omega$ and $\pi_{M}^{*} P_{i}$, respectively. Since $\theta^{(0)}=\pi_{0}^{*} \theta_{L}, F_{\theta^{(0)}}=0$, $\Delta^{0}=\{1\} \subset \mathbb{R}, s^{*}$ hor $_{\theta^{(0)}}=s^{*}$ and $\pi_{M} \circ s=\mathrm{id}_{M}$, we have

$$
\begin{aligned}
& \int_{\Delta^{0}} \operatorname{Car}^{\theta^{(0)}}\left(\pi_{M}^{*} \Omega\right)=s^{*} \text { hor }_{\theta^{(0)}}\left(\pi_{M}^{*} \Omega\right)=s^{*} \pi_{M}^{*} \Omega=\Omega, \\
& \int_{\Delta^{0}} \operatorname{Car}^{\theta^{(0)}}\left(\pi_{M}^{*} P_{i}\right)=0 .
\end{aligned}
$$

Therefore, the $\Omega^{0, n+1}\left(G^{\bullet} \times M\right)$-component of $\mathscr{I}\left(\operatorname{Car}^{\theta}\left(\jmath\left(\pi_{M}^{*}\left(\Omega+\sum_{i} P_{i}\right)\right)\right)\right)$ is indeed the $n$-plectic form $\Omega$, and, in particular, $G$-invariant.

We now turn to the $\Omega^{1, n}\left(G^{\bullet} \times M\right)$-components. Since $\operatorname{Car}^{\theta^{(1)}}\left(\pi_{M}^{*} \Omega\right) \in \Omega^{0, n+1}\left(\Delta^{1} \times E_{1} G \times\right.$ $M)^{G}$, we have

$$
\int_{\Delta^{1}} \operatorname{Car}^{\theta^{(1)}}\left(\pi_{M}^{*} \Omega\right)=0
$$


Thus, the homotopy moment map is constructed from

$$
\int_{\Delta^{1}} \operatorname{Car}^{\theta^{(1)}}\left(\pi_{M}^{*} P_{i}\right)=\int_{\Delta^{1}}\left\langle s^{*} F_{\theta^{(1)}}^{i}, s^{*} \operatorname{hor}_{\theta^{(1)}} \pi_{M}^{*} P_{i}\right\rangle .
$$

We will now compute this explicitly, and also show that it defines a $G$-invariant $n$-form on $G \times M$, so that we can apply the second part of Proposition 3.3.10.

Denote by $I: G \rightarrow G$ the map $g \mapsto g^{-1}$ and let $\theta_{R} \in \Omega^{1}(G, \mathfrak{g})$ be the right-invariant Maurer-Cartan form. The differential of the section $s: G \times M \rightarrow G^{2} \times M$ from (3.40) is

$$
\left.d s\right|_{(g, p)}(\tilde{x}, w)=\left(0, d I(\tilde{x}), d L_{g}(w)-\left.v_{\theta_{R}(\tilde{x})}^{G}\right|_{g p}\right) \text { for } \tilde{x} \in T_{g} G, w \in T_{p} M
$$

From (3.38) and (3.43) we obtain

$$
\begin{aligned}
& s^{*} F_{\theta^{(1)}}=-d t_{1} \wedge \pi_{G}^{*} \theta_{R}-\frac{t_{0} t_{1}}{2} \pi_{G}^{*}\left[\theta_{R}, \theta_{R}\right], \\
& s^{*} F_{\theta^{(1)}}^{i}=(-1)^{i} i \frac{\left(t_{0} t_{1}\right)^{i-1}}{2^{i-1}} d t_{1} \wedge \pi_{G}^{*}\left(\theta_{R} \wedge\left[\theta_{R}, \theta_{R}\right]^{i-1}\right)+\left(-\frac{t_{0} t_{1}}{2} \pi_{G}^{*}\left[\theta_{R}, \theta_{R}\right]\right)^{i} .
\end{aligned}
$$

On $E_{1} G \times M=G^{2} \times M$, the horizontal projection for the connection $\theta^{(1)}$ is given by

$$
\begin{aligned}
T_{\left(g_{0}, g_{1}, p\right)}\left(G^{2} \times M\right) & \rightarrow T_{\left(g_{0}, g_{1}, p\right)}\left(G^{2} \times M\right), \\
\left(\tilde{x}_{0}, \tilde{x}_{1}, w^{\prime}\right) & \mapsto\left(\tilde{x}_{0}, \tilde{x}_{1}, w^{\prime}\right)-v_{A\left(\tilde{x}_{0}, \tilde{x}_{1}, w^{\prime}\right)}^{G^{2} \times M}=\left(\tilde{x}_{0}, \tilde{x}_{1}, w^{\prime}\right)-v_{t_{0} \theta_{L}\left(\tilde{x}_{0}\right)+t_{1} \theta_{L}\left(\tilde{x}_{1}\right)}^{G^{2}} .
\end{aligned}
$$

Here, $v^{G^{2} \times M}$ is the infinitesimal action for the diagonal action $G \curvearrowright G^{2} \times M$ from (3.10). In particular,

$$
d \pi_{M}\left(h_{\theta^{(1)}}\left(\tilde{x}_{0}, \tilde{x}_{1}, w^{\prime}\right)\right)=w^{\prime}-\left.t_{0} v_{\theta_{L}\left(\tilde{x}_{0}\right)}^{G}\right|_{p}-\left.t_{1} v_{\theta_{L}\left(\tilde{x}_{1}\right)}^{G}\right|_{p} .
$$

Combining (3.43) and (3.46), and using $\theta_{L}(d I(\tilde{x}))=-\theta_{R}(\tilde{x})$ as well as $t_{0}=1-t_{1}$ and $d L_{g^{-1}} v_{\theta_{R}(\tilde{x})}^{G}=v_{\theta_{L}(\tilde{x})}^{G}$, we have

$$
\begin{aligned}
d \pi_{M}\left(\operatorname{hor}_{\theta^{(1)}} d s(\tilde{x}, w)\right) & =d \pi_{M}\left(\operatorname{hor}_{\theta^{(1)}}\left(0, d I(\tilde{x}), d L_{g}(w)-\left.v_{\theta_{R}(\tilde{x})}^{G}\right|_{g p}\right)\right) \\
& =d L_{g}(w)-\left.v_{\theta_{R}(\tilde{x})}^{G}\right|_{g p}-\left.t_{1} v_{\theta_{L}(d i(\tilde{x}))}^{G}\right|_{g p}=d L_{g}(w)-\left.t_{0} v_{\theta_{R}(\tilde{x})}^{G}\right|_{g p} \\
& =d L_{g}\left(w-\left.t_{0} v_{\theta_{L}(\tilde{x})}^{G}\right|_{p}\right)
\end{aligned}
$$

for all $(\tilde{x}, w) \in T_{(g, p)}(G \times M)$. Using the $G$-invariance of $P_{i}$, i.e. $L_{g}^{*} P_{i}=A d_{g}^{\vee} P_{i}$, we have

$$
\begin{aligned}
& \left.s^{*} \operatorname{hor}_{\theta^{(1)}} \pi_{M}^{*} P_{i}\right|_{(g, p)}\left(\left(\tilde{x}_{1}, w_{1}\right), \ldots,\left(\tilde{x}_{n-2 i+1}, w_{n-2 i+1}\right)\right) \\
= & \left.P_{i}\right|_{g p}\left(d L_{g}\left(w_{1}-\left.t_{0} v_{\theta_{L}\left(\tilde{x}_{1}\right)}^{G}\right|_{p}\right), \ldots, d L_{g}\left(w_{n-2 i+1}-\left.t_{0} v_{\theta_{L}\left(\tilde{x}_{n-2 i+1}\right)}^{G}\right|_{p}\right)\right) \\
= & \left.L_{g}^{*} P_{i}\right|_{p}\left(w_{1}-\left.t_{0} v_{\theta_{L}\left(\tilde{x}_{1}\right)}^{G}\right|_{p}, \ldots, w_{n-2 i+1}-\left.t_{0} v_{\theta_{L}\left(\tilde{x}_{n-2 i+1}\right)}^{G}\right|_{p}\right) \\
= & \left.\left(A d_{g}^{\vee}\right)^{\otimes i} P_{i}\right|_{p}\left(w_{1}-\left.t_{0} v_{\theta_{L}\left(\tilde{x}_{1}\right)}^{G}\right|_{p}, \ldots, w_{n-2 i+1}-\left.t_{0} v_{\theta_{L}\left(\tilde{x}_{n-2 i+1}\right)}^{G}\right|_{p}\right) .
\end{aligned}
$$

Denoting the map $T_{g} G \oplus T_{p} M \ni(\tilde{x}, w) \mapsto w-\left.t_{0} v_{\theta_{L}(\tilde{x})}^{G}\right|_{p} \in T_{p} M$, as well as any tensor power of it by $\phi_{t_{0}}$, we have

$$
s^{*} \operatorname{hor}_{\theta^{(1)}} \pi_{M}^{*} P_{i}=\left(A d_{g}^{\vee}\right)^{\otimes i} P_{i} \circ \phi_{t_{0}}
$$


Combining (3.42), (3.45), (3.47) and $A d_{g^{-1}} \theta_{R}=\theta_{L}$, we have

$$
\left.\operatorname{Car}^{\theta^{(1)}}\left(\pi_{M}^{*} P_{i}\right)\right)=\left\langle(-1)^{i} i \frac{\left(t_{0} t_{1}\right)^{i-1}}{2^{i-1}} d t_{1} \wedge \pi_{G}^{*}\left(\theta_{L} \wedge\left[\theta_{L}, \theta_{L}\right]^{i-1}\right)+\left(-\frac{t_{0} t_{1}}{2} \pi_{G}^{*}\left[\theta_{L}, \theta_{L}\right]\right)^{i}, P_{i} \circ \phi_{t_{0}}\right\rangle .
$$

Since $\phi_{t_{0}}$ is invariant under the left action $(g,(t, h, p)) \mapsto(t, g h, p)$, this also proves that

$$
\operatorname{Car}^{\theta^{(1)}}\left(\pi_{M}^{*} P_{i}\right) \in \Omega^{n}\left(\Delta^{1} \times G \times M\right)^{G},
$$

where $G$ acts by the same action. Hence also

$$
\int_{\Delta^{1}} \operatorname{Car}^{\theta^{(1)}}\left(\pi_{M}^{*} P_{i}\right)=\int_{\Delta^{1}}(-1)^{i} i \frac{\left(t_{0} t_{1}\right)^{i-1}}{2^{i-1}} d t_{1} \wedge\left\langle\pi_{G}^{*}\left(\theta_{L} \wedge\left[\theta_{L}, \theta_{L}\right]^{i-1}\right), P_{i} \circ \phi_{t_{0}}\right\rangle \in \Omega^{1, n}\left(G^{\bullet} \times M\right)^{G} .
$$

However, recall that $\phi_{t_{0}}$ depends on $t_{0}=1-t_{1}$. For $x_{1}, \ldots, x_{k} \in \mathfrak{g}$ and $w_{1}, \ldots, w_{n-k} \in T_{p} M$ we have

$$
\begin{aligned}
& \left\langle\pi_{G}^{*}\left(\theta_{L} \wedge\left[\theta_{L}, \theta_{L}\right]^{i-1}\right), P_{i} \circ \phi_{t_{0}}\right\rangle\left(\left(x_{1}, 0\right), \ldots,\left(x_{k}, 0\right),\left(0, w_{1}\right), \ldots,\left(0, w_{n-k}\right)\right) \\
= & \sum_{\sigma \in S h}\left(-t_{0}\right)^{\sigma}(-1)^{k-2 i+1}\left\langle\theta_{L} \wedge\left[\theta_{L}, \theta_{L}\right]^{i-1}\left(x_{\sigma(1)}, \ldots, x_{\sigma(2 i-1)}\right), P_{i}\left(v_{x_{\sigma(2 i)}}^{G}, \ldots, v_{x_{\sigma(k)}}^{G}, w_{1}, \ldots, w_{n-k}\right)\right\rangle \\
= & \frac{k ! t_{0}^{k-2 i+1}}{(k-2 i+1) !}\left\langle\pi_{\wedge^{k}\left(\mathfrak{g}^{\vee}\right)} \iota_{\mathfrak{g}}^{k-2 i+1} P_{i}(\cdot \underbrace{[\cdot, \cdot], \ldots,[\cdot, \cdot]}_{i-1}), x_{1}, \ldots, x_{k}\right\rangle\left(w_{1}, \ldots, w_{n-k}\right) .
\end{aligned}
$$

Here, $S h=S h(2 i-1, k-2 i+1)$ denotes the set of $(2 i-1, k-2 i+1)$-shuffles, i.e. permutations $\sigma$, which satisfy $\sigma(\ell)<\sigma(\ell+1)$ for all $\ell \neq 2 i-1$. Combining this with (3.48), and since $\int_{0}^{1} t_{0}^{k-i} t_{1}^{i-1} d t_{1}=\frac{(i-1) !(k-i) !}{k !}$, we see that the image of $\Omega+\sum_{i=1}^{\left\lfloor\frac{n+1}{2}\right\rfloor} P_{i}$ in $C^{*, *}(\mathfrak{g}, M)$ is

$$
\tilde{f}:=\sum_{i=1}^{\left\lfloor\frac{n+1}{2}\right\rfloor} \check{r} \int_{\Delta^{1}} \operatorname{Car}^{\theta^{(1)}}\left(\pi_{M}^{*} P_{i}\right)=\sum_{k=1}^{n} \underbrace{\sum_{i=1}^{\left\lfloor\frac{k+1}{2}\right\rfloor} \frac{(-1)^{i} i !(k-i) !}{2^{i-1}(k-2 i+1) !} \pi_{\wedge^{k}\left(\mathfrak{g}^{\vee}\right)}\left(\iota_{\mathfrak{g}}^{k-2 i+1} P_{i}(\cdot, \underbrace{[\cdot, \cdot], \ldots,[\cdot, \cdot]}_{i-1})\right)}_{\tilde{f}_{k}} .
$$

With $f_{k}=\zeta(k) \tilde{f}_{k}$, this completes the proof.

3.3.28 Example. For a cocycle of the form $\Omega+P_{1} \in C_{G}^{n+1}(M)$, with $P_{1} \in\left(\mathfrak{g}^{\vee} \otimes\right.$ $\left.\Omega^{n-1}(M)\right)^{G}$, we recover the statement of [FRZ13, Thm. 6.3], i.e. the moment map is

$$
f_{k}=-\zeta(k) \pi_{\wedge^{k}\left(\mathfrak{g}^{\vee}\right)} \iota_{\mathfrak{g}}^{k-1} P_{1}=-\zeta(k) \iota_{\mathfrak{g}}^{k-1} P_{1}
$$

Note that by [FRZ13, Prop. 6.2], $\iota_{\mathfrak{g}}^{k-1} P_{1}$ is already skew-symmetric.

In particular, for $n=1$, a degree 2 cocycle is of the form $\Omega-\mu \in C_{G}^{2}(M)$, where $\Omega \in \Omega^{2}(M)$ is a pre-symplectic structure on $M$ and $\mu \in\left(\mathfrak{g}^{\vee} \otimes \Omega^{0}(M)\right)^{G}$ is a moment map. The homotopy moment map is the usual moment map

$$
f=f_{1}=\mu \text {. }
$$


3.3.29 Example. If $\Omega+\sum_{i=1}^{\left\lfloor\frac{n+1}{2}\right\rfloor} P_{i} \in C_{G}^{n+1}(M)$ is a degree $n+1$ cocycle in the Cartan model with $P_{i} \in\left(S^{i}\left(\mathfrak{g}^{\vee}\right) \otimes \Omega^{n-2 i+1}(M)\right)^{G}$, then the first components of the homotopy moment map are

$$
\begin{aligned}
& f_{1}=-P_{1}, \\
& f_{2}=-\pi_{\wedge^{2}\left(\mathfrak{g}^{\vee}\right)} \iota_{\mathfrak{g}} P_{1}, \\
& f_{3}=\pi_{\wedge^{3}\left(\mathfrak{g}^{\vee}\right)} \iota_{\mathfrak{g}}^{2} P_{1}-\pi_{\wedge^{3}\left(\mathfrak{g}^{\vee}\right)} P_{2}(\cdot,[\cdot, \cdot]), \\
& f_{4}=\pi_{\wedge^{4}\left(\mathfrak{g}^{\vee}\right)} \iota_{\mathfrak{g}}^{3} P_{1}-2 \pi_{\wedge^{4}\left(\mathfrak{g}^{\vee}\right)} \iota_{\mathfrak{g}} P_{2}(\cdot,[\cdot, \cdot]), \\
& f_{5}=-\pi_{\wedge^{5}\left(\mathfrak{g}^{\vee}\right)} \iota_{\mathfrak{g}}^{4} P_{1}+3 \pi_{\wedge^{5}\left(\mathfrak{g}^{\vee}\right)} \iota_{\mathfrak{g}}^{2} P_{2}(\cdot,[\cdot, \cdot])-3 \pi_{\wedge^{5}\left(\mathfrak{g}^{\vee}\right)} P_{3}(\cdot,[\cdot, \cdot],[\cdot, \cdot]),
\end{aligned}
$$

3.3.30 Example. If $M=p t$, then $C_{G}^{*}(M)=C_{G}^{*}(p t)=\left(S^{*}\left(\mathfrak{g}^{\vee}\right)\right)^{G}$, then the construction produces

$$
\begin{aligned}
& \left(S^{i}\left(\mathfrak{g}^{\vee}\right)\right)^{G} \rightarrow\left(\wedge^{2 i-1}\left(\mathfrak{g}^{\vee}\right)\right)^{G} \\
& P_{i} \mapsto \tilde{f}_{2 i-1}=(-1)^{i \frac{i !(i-1) !}{2^{i-1}}} \pi_{\wedge^{2 i-1}\left(\mathfrak{g}^{\vee}\right)}\left(P_{i}(\cdot, \underbrace{[\cdot, \cdot], \ldots,[\cdot, \cdot]}_{i-1})\right),
\end{aligned}
$$

which differs by an additional factor of $-i$ ! from the Cartan map defined in [Car51, Sec. 2] (also cf. [GHV76, Ch. VI Prop. IV]).

The following corollary shows how the homotopy moment map changes when the cocyle in the Cartan model is changed by a coboundary. This generalizes [CFRZ15, Lem. 7.5] and [FLGZ14, Prop. 7.11].

3.3.31 Corollary. Given a cocycle $\Omega+\sum_{i=0}^{\left\lfloor\frac{n+1}{2}\right\rfloor} P_{i} \in C_{G}^{n+1}(M)$ and a coboundary $d_{G} Q=$ $d_{G} \sum_{i=0}^{\left\lfloor\frac{n}{2}\right\rfloor} Q_{i} \in C_{G}^{n+1}(M)$ in the Cartan complex of $M$ with $Q \in C_{G}^{n}(M)$, then the homotopy moment map for the pre-n-plectic form $\Omega+d Q_{0}$ associated to $\Omega+\sum_{i=0}^{\left\lfloor\frac{n+1}{2}\right\rfloor} P_{i}+d_{G} Q$ is given by

$$
\tilde{f}^{\prime}=\tilde{f}+r\left(\partial Q_{0}\right)-\delta_{\mathfrak{g}} \tilde{f}^{Q}-\sum_{k=1}^{n-1}(-1)^{k} d \tilde{f}_{k}^{Q},
$$

where, $\tilde{f}$ is the homotopy moment map for the pre-n-plectic action on $(M, \Omega)$ associated to $\Omega+\sum_{i=0}^{\left\lfloor\frac{n+1}{2}\right\rfloor} P_{i}$ and $\tilde{f}^{Q}=\check{r}\left(\beta_{1}\right)$, where $\beta=\sum_{k=0}^{n} \beta_{k}$ is the image of $Q$ under the chain map from Proposition 3.3.25.

3.3.32 Remark. If $d Q_{0}=0, \tilde{f}^{Q}$ is a homotopy moment map for the pre- $(n-1)$-plectic $G$-action on $\left(M, Q_{0}\right)$. In this situation, $\tilde{f}$ and $\tilde{f}^{\prime}$ both define homotopy moment maps for the pre- $n$-plectic $G$-action on $(M, \Omega)$.

If $d Q_{0} \neq 0$, the form $Q_{0}$ is not a pre- $n$-plectic form. However, $\tilde{f}^{Q}$ is given by the same formula (cf. Theorem 3.3.27). 
Proof (of Corollary 3.3.31). Let $\beta_{0} \in \Omega^{n}(M)^{G} \subset \Omega^{1, n-1}\left(G^{\bullet} \times M\right)$ and $\beta_{1} \in \Omega^{n-1}(G \times$ $M)^{G} \subset \Omega^{1, n-1}\left(G^{\bullet} \times M\right)$ be the relevant components of the image of $Q$ under the chain map from Proposition 3.3.25. Since the preimage of $\Omega^{1, n}\left(G^{\bullet} \times M\right)$ under $\mathbf{d}$ is $\Omega^{0, n}\left(G^{\bullet} \times M\right) \oplus \Omega^{1, n-1}\left(G^{\bullet} \times M\right)$, only $\beta_{0}$ and $\beta_{1}$ will contribute to the homotopy moment map. The component of $\mathbf{d}\left(\beta_{0}+\beta_{1}\right)$ in $\Omega^{1, n}\left(G^{\bullet} \times M\right)$ is

$$
\partial \beta_{0}-d \beta_{1}
$$

Therefore,

$$
\tilde{f}^{\prime}=\tilde{f}+\check{r}\left(\partial \beta_{0}-d \beta_{1}\right) .
$$

The claim now follows from Corollary 3.3.13. 



\section{Chapter 4}

\section{The generalized Dirac operator}

In this chapter, we recall the definition of the generalized Dirac operator in dimensions three and four associated to a hyperkähler manifold with permuting action. This Dirac operator was introduced by Taubes [Tau99] for three-dimensional manifolds and by Pidstrygach [Pid04] for four-dimensional manifolds. As usual, the Dirac operator is a composition of a covariant derivative and a Clifford multiplication. We study the Dirac operator, its linearization and its behavior on manifolds with boundary.

\section{1 $\operatorname{Spin}_{\varepsilon}^{G}(m)$-structures and spinors}

Recall that for a compact Lie group $G$ and $\varepsilon \in G$ central with $\varepsilon^{2}=1$,

$$
\operatorname{Spin}_{\varepsilon}^{G}(m):=(\operatorname{Spin}(m) \times G) / \pm 1
$$

where \pm 1 is the order 2 subgroup generated by $(-1, \varepsilon)$.

In this chapter, we restrict ourselves to $m \in\{3,4\}$ and use the isomorphisms

$$
\begin{aligned}
& \operatorname{Spin}_{\varepsilon}^{G}(3) \cong(\operatorname{Sp}(1) \times G) / \pm 1, \\
& \operatorname{Spin}_{\varepsilon}^{G}(4) \cong\left(S p(1)_{+} \times S p(1)_{-} \times G\right) / \pm 1 .
\end{aligned}
$$

4.1.1 Remark. This generalizes $\operatorname{Spin}(m), \operatorname{Spin}^{c}(m)$ and $S O(m) \times G$.

4.1.2 Note. Then we have a short exact sequence

$$
1 \rightarrow\langle(1, \varepsilon)\rangle \rightarrow \operatorname{Spin}_{\varepsilon}^{G}(m) \stackrel{\lambda^{G}}{\rightarrow} S O(m) \times G / \varepsilon \rightarrow 1,
$$

where $\lambda^{G}: \operatorname{Spin}_{\varepsilon}^{G}(m) \rightarrow S O(m) \times G / \varepsilon$ is the quotient map, $\langle(1, \varepsilon)\rangle$ the (normal) subgroup of $\operatorname{Spin}_{\varepsilon}^{G}(m)$ generated by $[(1, \varepsilon)]=[(-1,1)] \in \operatorname{Spin}_{\varepsilon}^{G}(m)$ and $G / \varepsilon$ the quotient of $G$ by the subgroup generated by $\varepsilon$.

Let now $Q_{m} \rightarrow Z$ be a $\operatorname{Spin}_{\varepsilon}^{G}(m)$-structure on a oriented Riemannian manifold $Z$ of dimension $\operatorname{dim}(Z)=m$, i.e. $Q_{m}$ is a $\lambda_{G}$-reduction $\pi: Q_{m} \rightarrow P_{S O(m)} \times_{Z} P_{G / \varepsilon}$ of principal bundles, where $P_{S O(m)} \rightarrow Z$ is the bundle of oriented orthonormal frames and $P_{G / \varepsilon} \rightarrow Z$ is a principal $G / \varepsilon$-bundle. 
We will denote the components of $\pi$ by $\pi_{S O}: Q_{m} \rightarrow P_{S O(m)}$ and $\pi_{G / \varepsilon}: Q_{m} \rightarrow P_{G / \varepsilon}$.

Given a $\operatorname{Spin}_{\varepsilon}^{G}(m)$-structure on $Z$ and a hyperkähler manifold $M$ with permuting $\operatorname{Spin}_{\varepsilon}^{G}(m)$

4.1.3 Definition (spinor). A (generalized) spinor is a smooth $\operatorname{Spin}_{\varepsilon}^{G}(m)$-equivariant map $u: Q_{m} \rightarrow M$. We will denote the space of spinors by

$$
\mathscr{N}_{m}:=C^{\infty}\left(Q_{m}, M\right)^{\operatorname{Spin}_{\varepsilon}^{G}(m)} .
$$

4.1.4 Note. Note that a $\operatorname{Spin}(m)$-structure, a $\operatorname{Spin}^{c}(m)$-structure and a principal $G$ bundle are special cases of $\operatorname{Spin}_{\varepsilon}^{G}(m)$-structures and using the representations from Example 2.2.6 as hyperkähler manifold with permuting action, we recover the usual (positive) spinor bundles.

Using $C^{\infty}\left(Q_{m}, M\right)^{\operatorname{Spin}_{\varepsilon}^{G}(m)} \cong \Gamma\left(Z, Q_{m} \times_{\operatorname{Spin}_{\varepsilon}^{G}(m)} M\right)$, a spinor $u \in \mathscr{N}$ can also be interpreted as a section of the associate fibre bundle with fibre $M$, which generalizes the usual (positive) spinor bundles.

4.1.5 Remark (Connectors, cf. [KMS93]). Recall that a linear connection on a vector bundle $\pi: E \rightarrow M$ can also be described in terms of a connector, i.e. a smooth map $\mathcal{K}: T E \rightarrow E$ which satisfies $\mathcal{K}\left(\frac{d}{d t} v+\left.t w\right|_{t=0}\right)=w$ for all $v, w \in T_{x} E$ and which is a morphism of vector bundles for both vector bundle structures on $T E$ :
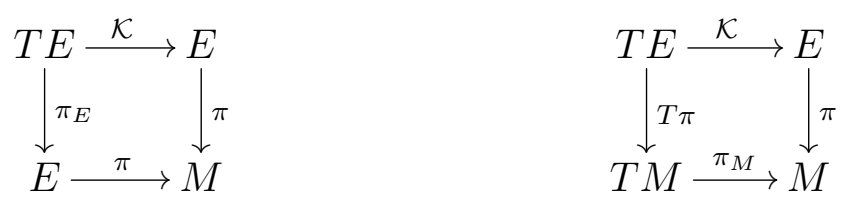

The horizontal subspace is then the kernel of $\mathcal{K}: T E \rightarrow E$, where $T E$ is considered as a vector bundle over $E$. A connector also defines a covariant derivative on all pullbacks of $E$ : Given $s \in \Gamma\left(N, f^{*} E\right) \cong C^{\infty}(N, E)_{f}$ for some smooth $f: N \rightarrow M$, we have $\nabla^{\mathcal{K}} s=\mathcal{K} \circ T s$.

Furthermore, let $\kappa_{M}: T T M \rightarrow T T M$ be the canonical flip, i.e. the unique smooth map satisfying $\left.\left.\frac{d}{d t} \frac{d}{d s} c(t, s)\right|_{s=0}\right|_{t=0}=\left.\left.\kappa_{M} \frac{d}{d s} \frac{d}{d t} c(t, s)\right|_{t=0}\right|_{s=0}$ for all smooth $c: \mathbb{R}^{2} \rightarrow M$.

The curvature of $\nabla^{\mathcal{K}}$ is given by

$$
F^{\mathcal{K}}(X, Y) s=\left(\mathcal{K} \circ(T \mathcal{K}) \circ \kappa_{T M}-\mathcal{K} \circ(T \mathcal{K})\right) T^{2} s \circ T X \circ Y
$$

for any section $s: M \rightarrow T M$, and vector fields $X, Y \in \Gamma(M, T M)$. The torsion 1-form $T^{\nabla^{\mathcal{K}}} \in \Omega^{2}(M, T M)$ is then given by

$$
T^{\nabla^{\mathcal{K}}}(v, w)=\left(\mathcal{K} \circ \kappa_{M}-\mathcal{K}\right) T v \circ w \text { for all } v, w \in \Gamma(M, T M) .
$$

For more details on connectors and proofs of the formulae for the curvature and torsion, we refer the reader to [KMS93, Thm. 37.15],

4.1.6 Remark. Note that $\mathscr{N}_{m}$ is a (infinite-dimensional) smooth manifold. If $Z$ is compact, it admits a natural Riemannian $L^{2}$-metric $g^{\mathscr{N}}$ (induced by $g^{M}$ ) and Levi-Civita connection $\nabla^{\mathscr{N}}$, whose connector $\mathcal{K}^{\mathscr{N}}: T T \mathscr{N}_{m} \rightarrow T \mathscr{N}_{m}$ is given by composition with the connector $\mathcal{K}^{M}: T T M \rightarrow T M$ of the Levi-Civita connection on $M$. Details are explained in [Cal10, App. A]. 


\subsection{Connections and covariant derivatives}

The Lie algebra $\mathfrak{s p i n}_{\varepsilon}^{G}(m)$ of $\operatorname{Spin}_{\varepsilon}^{G}(m)$ splits as a direct $\operatorname{sum} \mathfrak{s p i n}_{\varepsilon}^{G}(m) \cong \mathfrak{s o}(m) \oplus \mathfrak{g}$. Let $\varphi_{Z}$ be the Levi-Civita connection on $P_{S O(m)} \rightarrow Z$.

4.2.1 Definition. By $\mathscr{A}_{m}$ we denote the affine space of connections on $Q_{m} \rightarrow Z$ with $\mathfrak{s o}(m)$-component given by the lift of a chosen connection $\varphi_{Z}$ on $P_{S O(m)}$, i.e.

$$
\mathscr{A}_{m}:=\left\{A \in \mathscr{A}\left(Q_{m}\right) \mid \operatorname{pr}_{\mathfrak{s o}(m)} \circ A=\pi_{S O(m)}^{*} \varphi_{Z}\right\} .
$$

4.2.2 Remark. Most of the time, we use the Levi-Civita connection as $\varphi_{Z}$, since this is the natural choice of a connection on $P_{S O(m)}$. However, it is also possible to use another metric connection on $Z$ which is not torsion-free. This will be the case in Example 4.6.3.

4.2.3 Notation. Note that we have a commuting diagram of Lie algebras, where all maps are isomorphisms:

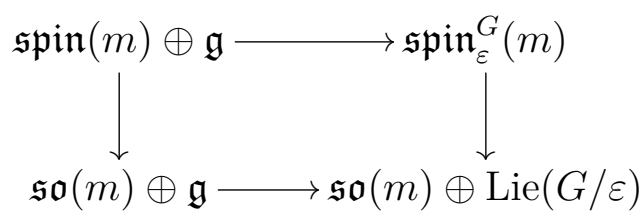

We will use this to identify $\mathfrak{s p i n}_{\varepsilon}^{G}(m) \cong \mathfrak{s o}(m) \oplus \mathfrak{g}$. The $\operatorname{maps} \operatorname{pr}_{\mathfrak{s o}(m)}: \mathfrak{s p i n} \mathfrak{n}_{\varepsilon}^{G}(m) \rightarrow \mathfrak{s o}(m)$ and $\operatorname{pr}_{\mathfrak{g}}: \mathfrak{s p i n}_{\varepsilon}^{G}(m) \rightarrow \mathfrak{g}$ will denote the above isomorphims composed with either of the projections to the two summands of $\mathfrak{s o}(m) \oplus \mathfrak{g}$.

4.2.4 Remark. Note that in the case of $G=S^{1}$, it is sometimes convenient to use the isomorphism $S^{1} / \pm 1 \cong S^{1},[z] \mapsto z^{2}$ and the induced isomorphims of Lie algebras $\operatorname{Lie}\left(S^{1} / \pm 1\right) \cong \operatorname{Lie}\left(S^{1}\right) \cong i \mathbb{R}$. In this case, the bottom map in the diagram is $\operatorname{id}_{\mathfrak{s o}(m)} \times 2 \mathrm{id}_{i \mathbb{R}}$. This factor of 2 often appear in the literature on Seiberg-Witten theory.

\subsubsection{Gauge group}

We can now study the automorphism group of a $\operatorname{Spin}_{\varepsilon}^{G}(m)$-structure.

4.2.5 Definition. Let $Q_{m} \rightarrow Z$ be a $\operatorname{Spin}_{\varepsilon}^{G}(m)$-structure on $Z$. The gauge group of the $\operatorname{Spin}(m)$-equivariant principal $G$-bundle $Q_{m} \rightarrow P_{S O(m)}$ is denoted by $\mathscr{G}_{m}$, i.e.

$$
\mathscr{G}_{m}:=C^{\infty}\left(Q_{m}, G\right)^{\operatorname{Spin}_{\varepsilon}^{G}(m)} \cong \mathscr{G}\left(Q_{m} \rightarrow P_{S O(m)}\right)^{\operatorname{Spin}_{\varepsilon}^{G}(m)} \subset \operatorname{Aut}\left(Q_{m}\right) .
$$

We will refer to $\mathscr{G}_{m}$ as the gauge group. It naturally acts on $\mathscr{N}_{m}$ and $\mathscr{A}_{m}$.

\subsection{Covariant derivative}

Let $Q \rightarrow P_{S O(m)} \rightarrow Z$ be a reduction of the principal bundle of oriented orthonormal frames, with structure group $H$. We are particularly interested in the case when $H=$ $\operatorname{Spin}_{\varepsilon}^{G}(m)$ and $Q$ is a $\operatorname{Spin}_{\varepsilon}^{G}(m)$-structure. 
4.3.1 Definition. For a connection 1-form $A \in \mathscr{A}(Q)$ which lifts a connection $\varphi_{Z}$ on $P_{S O(m)}$, we define a covariant derivative

$$
\begin{aligned}
& d_{A}^{M}: C^{\infty}(Q, M)^{H} \rightarrow C^{\infty}\left(Q,\left(\mathbb{R}^{m}\right)^{\vee} \otimes T M\right)^{H}, \\
&\left\langle\left(d_{A}^{M} u\right)(p), w\right\rangle:=T u(\tilde{w}) \text { for } w \in \mathbb{R}^{n} .
\end{aligned}
$$

Here $\tilde{w} \in T_{p} Q$ is the horizontal lift of $\pi_{S O}(p)(w) \in T_{\pi_{Z}(p)} Z$.

We will also use the following variation of the concept of covariant derivative: Consider a $H$-equivariant vector bundle $E \rightarrow M$ with a fixed $H$-equivariant connection on $E$ and the corresponding connector $\mathcal{K}: T E \rightarrow E$. We define

$$
\begin{aligned}
& d_{A, \mathcal{K}}^{E}: C^{\infty}(Q, E)^{H} \stackrel{d_{A}^{E}}{\longrightarrow} C^{\infty}\left(Q,\left(\mathbb{R}^{m}\right)^{\vee} \otimes T E\right)^{H} \stackrel{\operatorname{id}_{\left(\mathbb{R}^{m}\right) \vee \otimes \mathcal{K}}}{\longrightarrow} C^{\infty}\left(Q,\left(\mathbb{R}^{m}\right)^{\vee} \otimes E\right)^{H}, \\
& d_{A, \mathcal{K}}^{E} v:=\left(\operatorname{id}_{\left(\mathbb{R}^{m}\right)^{\vee}} \otimes \mathcal{K}\right) \circ d_{A}^{E} v, v \in C^{\infty}(Q, E)^{H} .
\end{aligned}
$$

Here $d_{A}^{E}: C^{\infty}(Q, E)^{H} \rightarrow C^{\infty}\left(Q,\left(\mathbb{R}^{m}\right)^{\vee} \otimes T E\right)^{H}$ is the covariant derivative defined above for the total space of the vector bundle $E \rightarrow M$.

4.3.2 Example. For a representation $M=V$ of $H$ the map $d_{A}^{M}$ is the usual covariant exterior derivative if we identify $C^{\infty}\left(Q,\left(\mathbb{R}^{n}\right)^{\vee} \otimes V\right)^{H} \cong \Omega^{1}(Q, V)_{h o r}^{H}$.

4.3.3 Remark. Note that $d_{A}^{M}$ is a smooth section of the infinite-dimensional vector bundle $C^{\infty}\left(Q,\left(\mathbb{R}^{m}\right)^{\vee} \otimes T M\right)^{H} \rightarrow C^{\infty}(Q, M)^{H}, \alpha \mapsto \pi_{M} \circ \alpha$ (cf. [Cal10, Lem. 3.4.4]).

Similarly, $d_{A, \mathcal{K}}^{E}$ is a morphism of infinite-dimensional vector bundles

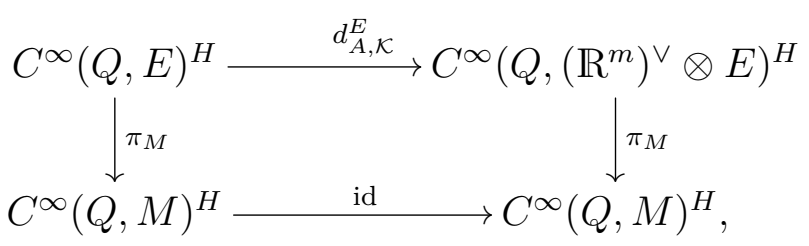

where $\pi_{M}$ denotes the composition with the projection to $M$. In particular, we can restrict our attention to the fibres over $u \in C^{\infty}(Q, M)^{H}$ : The following commutative diagram defines a covariant derivative $\nabla^{A, \mathcal{K}}$ on the vector bundle $\pi_{!} u^{*} E:=u^{*} E / H \rightarrow Q / H=Z$ :

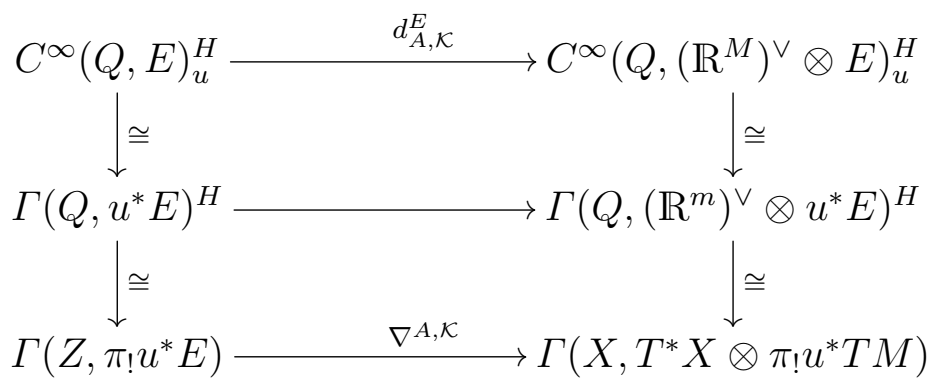

The following Lemma generalizes [Cal10, Lem. 3.4.4] and states some essential properties of the covariant derivative: 
4.3.4 Lemma (Properties of the covariant derivative). Let $A \in \mathscr{A}(Q)$ lifting a connection $\varphi_{Z}$ on $P_{S O(m)}$ and $\mathcal{K}$ the connector of a connection on $T M \rightarrow M$ with vanishing torsion. Then the covariant derivative

$$
d_{A}^{M}: C^{\infty}(Q, M)^{H} \rightarrow C^{\infty}\left(Q,\left(\mathbb{R}^{m}\right)^{\vee} \otimes T M\right)^{H}
$$

is smooth and we have

1. $T d_{A}^{M}=\left(\operatorname{id}_{\left(R^{m}\right) \vee} \otimes \kappa_{M}\right) \circ d_{A}^{T M}$,

2. $\left(\operatorname{id}_{\left(R^{m}\right) \vee} \otimes \mathcal{K}\right) \circ T d_{A}^{M}=d_{A, \mathcal{K}}^{T M}$,

3. $T d_{A, \mathcal{K}^{E}}^{E}=\left(\operatorname{id}_{\left(R^{m}\right)^{\vee}} \otimes\left(\left(T \mathcal{K}^{E}\right) \circ \kappa_{E}\right)\right) \circ d_{A}^{T E}$, where $\mathcal{K}^{E}$ is a connector on $E$,

4. $\mathcal{K}^{E} T d_{A, \mathcal{K}^{E}}^{E}=\left(\operatorname{id}_{\left(R^{m}\right)^{\vee}} \otimes\left(\mathcal{K}^{E} \circ\left(T \mathcal{K}^{E}\right) \circ \kappa_{E}\right)\right) \circ d_{A}^{T E}$, where $\mathcal{K}^{E}$ is a connector on $E$.

5. For a H-equivariant smooth map $f: M \rightarrow M^{\prime}$ we have $T f \circ d_{A}^{M} u=d_{A}^{M^{\prime}}(f \circ u)$.

6. If $\hat{\chi} \in \Gamma(M, T M)$ satisfies $\nabla^{\mathcal{K}^{M}} \hat{\chi}=\mathrm{id}_{T M}$, then $d_{A, \mathcal{K}}^{T M}(\chi \circ u)=d_{A}^{M} u$.

Proof. Proofs for the first two claims for $H=\operatorname{Spin}_{\varepsilon}^{G}(m)$ can be found in [Cal10, Lem. 3.4.4], but the exact same proofs works in the case of an arbitrary Lie group $H$. The third and fourth claim are immediate consequences of the definition of $d_{A, \mathcal{K}^{E}}^{E}$ and the second claim. The fifth item is obvious from the definition of $d_{A}^{M}$.

We now proof the sixth item: From $\mathcal{K}^{M} T \hat{\chi}=\nabla^{\mathcal{K}^{M}} \hat{\chi}=\mathrm{id}_{T M}$ and the fifth item, we obtain

$$
\left\langle\left(d_{A, \mathcal{K}}^{T M}(\hat{\chi} \circ u)\right)(p), w\right\rangle=\mathcal{K} T \hat{\chi} T u(\tilde{w})=T u(\tilde{w})=\left\langle\left(d_{A}^{M} u\right)(p), w\right\rangle
$$

for all $w \in \mathbb{R}^{m}, p \in Q$ and where $\tilde{w} \in T_{p} Q$ is the horizontal lift of $\pi_{S O}(p)(w) \in T_{\pi_{Z}(p)} Z . \square$

4.3.5 Remark. Under the isomorphism $C^{\infty}\left(Q,\left(\mathbb{R}^{m}\right)^{\vee} \otimes T M\right)^{H} \cong \Omega^{1}(Q, T M)_{h o r}^{H}$, the covariant exterior derivative $d_{A}^{M} u$ corresponds to $\operatorname{pr}_{\mathscr{H}_{A}}^{*} T u$.

\subsection{Clifford multiplication and hyperkähler manifolds}

In this section, we recall the definition of the Clifford multiplication used for the generalized Dirac operator. Note that in contrast to other references on the topic (e.g. [Pid04], [Hay06], [Sch10], [Cal10]), we allow $S p(1)_{-}$to act non-trivially (hyperkähler) in the 4-dimensional case. To generalize Clifford multiplication to the case of spinors with values in a hyperkähler manifold $M$, we need a $\operatorname{Spin}_{\varepsilon}^{G}(m)$-equivariant bundle of $C l_{m}$-modules.

In dimension 3, this is given by the scalar multiplication on $T M$ :

$$
\begin{gathered}
c_{3}: \mathbb{R}^{3} \otimes T M \cong \operatorname{Im}(\mathbb{H}) \otimes T M \rightarrow T M, \\
h \otimes v \mapsto \mathcal{I}_{\bar{h}} v
\end{gathered}
$$


defines a $\operatorname{Spin}_{\varepsilon}^{G}(m)$-equivariant $C l_{3}$-module structure on $T M$ (cf. [Cal10, Lem. 3.5.1]).

In dimension 4 , the situation is slightly more complicated. Let us first consider the $S p(1)_{+} \times S p(1)_{-} \times G$-equivariant vector bundle $E \rightarrow M$, which is isomorphic to $T M$ as a vector bundle and carries the following $S p(1)_{+} \times S p(1)_{-} \times G$-action:

$$
\begin{aligned}
S p(1)_{+} \times S p(1)_{-} \times G & \curvearrowright E, \\
\left(\left(q_{+}, q_{-}, g\right), v\right) & \mapsto \mathcal{I}_{\overline{q_{+}}}\left(q_{+}\right)_{*}\left(q_{-}\right)_{*} g_{*} v .
\end{aligned}
$$

This is a well-defined action since the $S p(1)_{+}$-action on $M$ is permuting and, therefore, $\left(q_{+}\right)_{*} \mathcal{I}_{\overline{q_{+}}}=\mathcal{I}_{\overline{q_{+}}}\left(q_{+}\right)_{*}$ commutes with the complex structures on $T M$.

Since $T M$ is a bundle of $C l_{4}^{0}=C l_{3}$-modules,

$$
\widehat{T M}:=C l_{4} \otimes_{C l_{3}} E
$$

is a natural bundle of $C l_{4}$-module constructed from $T M$. Furthermore, this $C l_{4}$-module structure is $\operatorname{Spin}_{\varepsilon}^{G}(4)$-equivariant with the $\operatorname{Spin}_{\varepsilon}^{G}(4)$-action induced by the $S p(1)_{+} \times$ $S p(1)_{-} \times G$-action on $E$ and $\operatorname{Spin}(4) \subset C l_{4} \curvearrowright C l_{4}$. Additionally, $\widehat{T M}$ has a $\mathbb{Z} / 2 \mathbb{Z}$ grading induced by $C l_{4}=C l_{4}^{0} \oplus C l_{4}^{1}$ with even and odd parts

$$
\widehat{T M}^{0}=C l_{4}^{0} \otimes_{C l_{4}^{0}} E \cong T M, \quad \widehat{T M}^{1}=C l_{4}^{1} \otimes_{C l_{4}^{0}} E
$$

where $\widehat{T M}^{1}$ is again $T M$ as a vector bundle and carries the following $\operatorname{Spin}_{\varepsilon}^{G}(4)$-action:

$$
\begin{aligned}
\operatorname{Spin}_{\varepsilon}^{G}(4) \cong\left(\operatorname{Sp}(1)_{+} \times S p(1)_{-} \times G\right) / \pm 1 & \curvearrowright \widehat{T M}^{1}, \\
\left(\left[\left(q_{+}, q_{-}, g\right)\right], v\right) & \mapsto \mathcal{I}_{q_{-}} \mathcal{I}_{\overline{q_{+}}}\left(q_{+}\right)_{*}\left(q_{-}\right)_{*} g_{*} v .
\end{aligned}
$$

In particular, we have a Clifford multiplication

$$
c_{4}: \mathbb{R}^{4} \otimes \widehat{T M} \rightarrow \widehat{T M}
$$

which interchanges the even part $T M$ and the odd part $\widehat{T M}^{1}$ of $\widehat{T M}$. Under the isomorphism End $(\widehat{T M}) \cong$ End $(T M \oplus \underline{T M})$, the Clifford multiplication on $\widehat{T M}$ corresponds to the map

$$
e_{0} \mapsto\left(\begin{array}{cc}
0 & -\mathrm{id}_{T M} \\
\operatorname{id}_{T M} & 0
\end{array}\right) \quad \text { and } \quad e_{\ell} \mapsto\left(\begin{array}{cc}
0 & c_{3}\left(e_{\ell}\right) \\
c_{3}\left(e_{\ell}\right) & 0
\end{array}\right) \text { for } \ell \in\{1,2,3\}
$$

In particular, $c_{4}\left(e_{0}\right)^{-1} c_{4}\left(e_{\ell}\right)=c_{3}\left(e_{\ell}\right) \in \operatorname{End}(T M)=\operatorname{End}\left(\widehat{T M}^{0}\right)$ for $\ell \in\{1,2,3\}$.

4.4.1 Remark. Also note that $\widehat{T M} \cong\left[\left(S^{+} \oplus S^{-}\right) \otimes_{\mathbb{C}} E\right]_{r}$ for some real structure $r$ and $T M \cong\left[S^{+} \otimes_{\mathbb{C}} E\right]_{r}, \widehat{T M}^{1} \cong\left[S^{-} \otimes E\right]_{r}$. These isomorphisms have been used in [Pid04] and [Hay06]. The Clifford multiplication is then induced by the usual Clifford multiplication $C l_{4}: \mathbb{R}^{4} \otimes S^{ \pm} \rightarrow S^{\mp}$. 
4.4.2 Example. For the $\operatorname{Spin}^{(c)}(m)$-representation from Example 2.2.6, we recover the usual Clifford multiplication on the (positive) spinor module. In all considered cases, the Clifford multiplication is given by

$$
\begin{aligned}
\mathbb{H} \otimes \mathbb{H} & \rightarrow \mathbb{H}, \\
h \otimes h^{\prime} & \mapsto \bar{h} h^{\prime} .
\end{aligned}
$$

This can be interpreted as a homomorphism of $\operatorname{Spin}(m)$ or $\operatorname{Spin}^{c}(m)$-representations

$$
\begin{array}{llll}
\mathbb{R}^{3} \otimes S \rightarrow S & \text { and } & \mathbb{R}^{3} \otimes W \rightarrow W & \text { for } m=3, \\
\mathbb{R}^{4} \otimes S^{+} \rightarrow S^{-} & \text {and } & \mathbb{R}^{4} \otimes W^{+} \rightarrow W^{-} & \text {for } m=4,
\end{array}
$$

where in the three-dimensional case, we take the restriction of the above homomorphism to $\operatorname{Im}(\mathbb{H}) \otimes \mathbb{H}$.

4.4.3 Remark. Since the Clifford multiplication $c_{m}: \mathbb{R}^{m} \otimes T M \rightarrow T M$ is given by scalar multiplication, it is parallel with respect to the Levi-Civita connection on $M$, i.e. $\nabla^{M}\left(c_{m}\right)=0$. This can be written as $\mathcal{K} T\left(c_{m}\right)=c_{m} \circ\left(\operatorname{id}_{\left(R^{m}\right)} \vee \otimes \mathcal{K}\right)$, where $\mathcal{K}$ is the connector of the Levi-Civita connection $\nabla^{M}=\nabla^{\mathcal{K}}$ (cf. [Cal10, Lem. 3.5.4]).

\subsection{Dirac operator}

We define the Dirac operator as the composition of the covariant derivative and Clifford multiplication.

\subsubsection{Definition (Dirac operator).}

The (three-dimensional) Dirac operator $\mathscr{D}_{A}$ for a connection $A \in \mathscr{A}_{3}$ is defined to be the composition

$$
\begin{aligned}
& C^{\infty}\left(Q_{3}, M\right)^{\operatorname{Spin}_{\varepsilon}^{G}(3)} \stackrel{d_{A}^{M}}{\longrightarrow} C^{\infty}\left(Q_{3},\left(\mathbb{R}^{3}\right)^{\vee} \otimes T M\right)^{\operatorname{Spin}_{\varepsilon}^{G}(3)} \stackrel{c_{3}}{\longrightarrow} C^{\infty}\left(Q_{3}, T M\right)^{\operatorname{Spin}_{\varepsilon}^{G}(3)}, \\
& \mathscr{D}_{A} u:=c_{3}\left(d_{A}^{M} u\right) .
\end{aligned}
$$

The (four-dimensional) Dirac operator $\mathscr{D}_{A}^{+}$for a connection $A \in \mathscr{A}_{4}$ is defined to be the composition

$$
\begin{aligned}
& C^{\infty}\left(Q_{4}, M\right)^{\operatorname{Spin}_{\varepsilon}^{G}(4)} \stackrel{d_{A}^{M}}{\longrightarrow} C^{\infty}\left(Q_{4},\left(\mathbb{R}^{4}\right)^{\vee} \otimes T M\right)^{\operatorname{Spin}_{\varepsilon}^{G}(4)} \stackrel{c_{4}}{\longrightarrow} C^{\infty}\left(Q_{4}, \widehat{T M}^{1}\right)^{\operatorname{Spin}_{\varepsilon}^{G}(4)}, \\
& \mathscr{D}_{A}^{+} u:=c_{4}\left(d_{A}^{M} u\right) .
\end{aligned}
$$

4.5.2 Note. The Dirac operators $\mathscr{D}_{A}$ and $\mathscr{D}_{A}^{+}$are sections of (infinite-dimensional) vector bundles over $C^{\infty}\left(Q_{m}, M\right)^{\operatorname{Spin}_{\varepsilon}^{G}(m)}$, which are given by composition with the projection $T M \rightarrow M$.

\subsubsection{The linearized Dirac operator}

We will now linearize the Dirac operator in three dimensions. Let $Q_{m} \rightarrow Z$ be a $\operatorname{Spin}_{\varepsilon}^{G}(m)$ structure on a compact oriented Riemannian manifold $Z$ of dimension $m \in\{3,4\}$. 
4.5.3 Definition. Using the connector $\mathcal{K}: T T M \rightarrow T M$ for the Levi-Civita connection on $M$, we define the linearized Dirac operator $\mathscr{D}_{A}^{\text {lin,u }}$ in dimension 3 (at $u \in$ $\left.C^{\infty}\left(Q_{3}, M\right)^{\operatorname{Spin}_{\varepsilon}^{G}(3)}\right)$ to be

$$
\begin{aligned}
\mathscr{D}_{A}^{l i n, u}: C^{\infty}\left(Q_{3}, T M\right)_{u}^{\operatorname{Spin}_{\varepsilon}^{G}(3)} & \rightarrow C^{\infty}\left(Q_{3}, T M\right)_{u}^{\operatorname{Spin}_{\varepsilon}^{G}(3)}, \\
v & \mapsto \mathcal{K} \circ T_{u} \mathscr{D}_{A}(v),
\end{aligned}
$$

and the linearized Dirac operator $\mathscr{D}_{A}^{l i n, u,+}$ in dimension $4\left(\right.$ at $\left.u \in C^{\infty}\left(Q_{4}, M\right)^{\operatorname{Spin}_{\varepsilon}^{G}(4)}\right)$ to be

$$
\begin{aligned}
\mathscr{D}_{A}^{l i n, u,+}: C^{\infty}\left(Q_{4}, T M\right)_{u}^{\operatorname{Spin}_{\varepsilon}^{G}(4)} & \rightarrow C^{\infty}\left(Q_{4}, \widehat{T M}^{1}\right)_{u}^{\operatorname{Spin}_{\varepsilon}^{G}(4)}, \\
v & \mapsto \mathcal{K} \circ T_{u} \mathscr{D}_{A}^{+}(v) .
\end{aligned}
$$

Also, with an eye to Proposition 4.5.10, we define

$$
\begin{aligned}
\mathscr{D}_{A}^{l i n, u,-}: C^{\infty}\left(Q_{4}, \widehat{T M}^{1}\right)_{u}^{\operatorname{Spin}_{\varepsilon}^{G}(4)} & \rightarrow C^{\infty}\left(Q_{4}, T M\right)_{u}^{\operatorname{Spin}_{\varepsilon}^{G}(4)}, \\
v & \mapsto c_{4} \circ{\widehat{d_{A, \mathcal{K}}^{T M}}}^{1}(v) .
\end{aligned}
$$

We also denote

$$
\begin{aligned}
& \mathscr{D}_{A}^{l i n, u, *}: C^{\infty}\left(Q_{3}, T M\right)^{\operatorname{Spin}_{\varepsilon}^{G}(3)} \rightarrow C^{\infty}\left(Q_{3}, T M\right)^{\operatorname{Spin}_{\varepsilon}^{G}(3)} \\
& \mathscr{D}_{A}^{l i n, u, *} w:=\mathscr{D}_{A}^{l i n, u} w-c_{3}\left(\mathcal{T}^{\varphi} \otimes w\right),
\end{aligned}
$$

and

$$
\begin{aligned}
& \mathscr{D}_{A}^{l i n, u,+, *}: C^{\infty}\left(Q_{4}, \widehat{T M}^{1}\right)^{\operatorname{Spin}_{\varepsilon}^{G}(4)} \rightarrow C^{\infty}\left(Q_{4}, T M\right)^{\operatorname{Spin}_{\varepsilon}^{G}(4)} \text {, } \\
& \mathscr{D}_{A}^{\text {lin, },+, *} w:=\mathscr{D}_{A}^{\text {lin,u,-}} w-c_{4}\left(\mathcal{T}^{\varphi} \otimes w\right) .
\end{aligned}
$$

Here $\mathcal{T}^{\varphi} \in \Omega^{1}(Z) \cong C^{\infty}\left(Q_{m},\left(\mathbb{R}^{m}\right)^{\vee}\right)^{\operatorname{Spin}_{\varepsilon}^{G}(m)}$ denotes the torsion 1-form for the connection $\varphi$ on $P_{S O(m)}$ which $A$ lifts, i.e. $\mathcal{T}^{\varphi}(\eta)=-\operatorname{tr}\left(\iota_{\eta} T^{\varphi}\right)$, where $T^{\varphi} \in \Omega^{2}(Z, T Z)$ is the torsion of $\varphi$.

4.5.4 Remark. Note that if $Z$ is compact, then the linearized Dirac operator $\mathscr{D}_{A}^{l i n, u}$ is the covariant derivative $\nabla^{\mathscr{N}} \mathscr{D}_{A}$ at $u \in \mathscr{N}_{3}$, where $\nabla^{\mathscr{N}}$ is the metric compatible covariant derivative corresponding to the connector $\mathcal{K}^{\mathscr{N}}$ in Remark 4.1.6.

4.5.5 Remark. Note that in the 4-dimensional case, $\mathcal{K}: T \widehat{T M}^{1} \rightarrow \widehat{T M}^{1}$ is $\operatorname{Spin}_{\varepsilon}^{G}(4)$ equivariant, since we are using the Levi-Civita connection, which is $\operatorname{Spin}_{\varepsilon}^{G}(4)$-invariant, since $\operatorname{Spin}_{\varepsilon}^{G}(4)$ acts isometrically.

4.5.6 Remark. Note that in the definition of $\mathscr{D}_{A}^{\text {lin,u,--}}$, we are using the Clifford multiplication $\left(\mathbb{R}^{4}\right)^{\vee} \otimes \widehat{T M}^{1} \rightarrow T M$ obtained by restricting the action of the Clifford algebra on $\widehat{T M}:=C l_{4} \otimes_{C l_{4}^{0}} E \cong T M \oplus \widehat{T M}^{1}$. 
4.5.7 Remark. Note that from Remark 4.4.3, we immediately obtain

$$
\begin{aligned}
\mathscr{D}_{A}^{l i n, u,(+)}(v) & =\mathcal{K} \circ T_{u} \mathscr{D}_{A}^{(+)}(v)=\mathcal{K} \circ T\left(c_{m}\right) T\left(d_{A}^{M}\right)(v)=c_{m} \circ\left(\operatorname{id}_{\left(\mathbb{R}^{3}\right) \vee} \otimes \mathcal{K}\right) \circ T\left(d_{A}^{M}\right)(v) \\
& =c_{m} \circ d_{A, \mathcal{K}}^{T M}(v) .
\end{aligned}
$$

and therefore $\mathscr{D}_{A}^{l i n, u}=c_{3} \circ d_{A, \mathcal{K}}^{T M}$ and $\mathscr{D}_{A}^{l i n, u,+}=c_{4} \circ d_{A, \mathcal{K}}^{T M}$ are usual Dirac operators for the connection $\nabla^{A, \mathcal{K}}$ on $\pi ! u^{*} T M$ described in Remark 4.3.3.

In some cases, the generalized Dirac operator $\mathscr{D}_{A}$ is determined by its linearization.

4.5.8 Corollary. Let $\hat{\chi} \in \Gamma(M, T M)^{\operatorname{Spin}_{\varepsilon}^{G}(m)}$ be a $\operatorname{Spin}_{\varepsilon}^{G}(m)$-equivariant vector field satisfying $\nabla^{M} \hat{\chi}=\mathrm{id}_{\Gamma(M, T M)}$, where $\nabla^{M}$ is the Levi-Civita connection on $M$. Then

$$
\mathscr{D}_{A}^{l i n, u}(\hat{\chi} \circ u)=c_{3}\left(d_{A, \mathcal{K}}^{T M}(\hat{\chi} \circ u)\right)=c_{3}\left(d_{A}^{M} u\right)=\mathscr{D}_{A}(u),
$$

and

$$
\mathscr{D}_{A}^{l i n, u,+}(\hat{\chi} \circ u)=c_{4}\left(d_{A, \mathcal{K}}^{T M}(\hat{\chi} \circ u)\right)=c_{4}\left(d_{A}^{M} u\right)=\mathscr{D}_{A}^{+}(u),
$$

where $u \in C^{\infty}\left(Q_{m}, M\right)^{\operatorname{Spin}_{\varepsilon}^{G}(m)}$ with $m=3$ or $m=4$, respectively.

4.5.9 Remark. The special case $\rho_{2} \equiv 0$ and $\hat{\chi}=\chi_{0}$ was also discussed in [Sch10, Cor. 4.6.2], [Cal10, Lem. 3.6.9].

However, as we have seen in Remark 2.2.48, even if $\rho_{2} \not \equiv 0$, a vector field $\hat{\chi}$ with $\nabla \hat{\chi}=\mathrm{id}_{T M}$, or equivalently, a hyperkähler potential, may still exist. Examples can be obtained by modifying permuting actions with $\rho_{2} \equiv 0$.

The following Lemma reflects the fact that $\mathscr{D}_{A}^{l i n, u,(+)}$ is a usual Dirac operator acting on sections in $\pi_{!} u^{*} T M:=u^{*} T M / \operatorname{Spin}_{\varepsilon}^{G}(3)$ (or $\pi_{!} u^{*} \widehat{T M}$ ). We also use $\pi_{!} h$ to denote the function on $Z$ which is induced by a $\operatorname{Spin}_{\varepsilon}^{G}(3)$-invariant function $h$ on $Q_{m}$. The 3-dimensional case with $\varphi$ the Levi-Civita connection was also discussed in [Cal10, Lem. 3.6.8].

4.5.10 Proposition. Let $Q_{m} \rightarrow P_{S O(m)} \rightarrow Z$ be a $\operatorname{Spin}_{\varepsilon}^{G}(m)$-structure on a compact oriented Riemannian manifold $Z$ of dimension $m \in\{3,4\}$, with boundary $\partial Z$. Let $A \in \mathscr{A}_{m}$ be a connection lifting a metric connection $\varphi$ with covariant derivative $\nabla$ on $Z$. Let $\mathcal{T}^{\nabla}(v):=-\operatorname{tr}\left(\iota_{v} T^{\nabla}\right)$ denote the torsion 1 -form obtained from the torsion tensor $T^{\nabla} \in \Omega^{2}(Z, T Z)$, where we think of $\mathcal{T}^{\nabla} \in C^{\infty}\left(Q,\left(\mathbb{R}^{m}\right)^{\vee}\right)^{\operatorname{Spin}_{\varepsilon}^{G}(m)}$. Then

1. for all $v, w \in C^{\infty}\left(Q_{3}, T M\right)_{u}^{\operatorname{Spin}_{\varepsilon}^{G}(3)}$ :

$$
\begin{aligned}
\left\langle\mathscr{D}_{A}^{l i n, u} v, w\right\rangle_{L^{2}} & =\left\langle v, \mathscr{D}_{A}^{l i n, u} w\right\rangle_{L^{2}}+\int_{Y} \operatorname{div}^{\nabla}\left(U_{v, w}\right) * 1 \\
& =\left\langle v, \mathscr{D}_{A}^{l i n, u, *} w\right\rangle_{L^{2}}-\int_{\partial Y} \pi ! g^{M}\left(v, c_{3}\left(f_{\overrightarrow{\mathfrak{n}}} \otimes w\right)\right) * 1
\end{aligned}
$$

where $\operatorname{div}^{\nabla}$ denotes the divergence with respect to the $S O(3)$-connection on $P_{S O(3)}$ which $A$ lifts and $U_{v, w} \in \Gamma(Y, T Y)$ is defined by $g^{Y}\left(U_{v, w}, Z\right)=-\pi_{!}\left(g^{M}\left(v, c_{3}\left(f_{Z} \otimes w\right)\right)\right)$ for $Z \in \Gamma(Y, T Y)$ and $f_{Z}: Q_{3} \rightarrow \mathbb{R}^{3}$ the corresponding $\operatorname{Spin}_{\varepsilon}^{G}(3)$-equivariant map. 
In particular, if $Y$ is closed and $A$ projects to the Levi-Civita connection on $Y$, or more generally, if $\int_{Y} \operatorname{div}^{\nabla}\left(U_{v, w}\right)=0$ for all $v, w \in C^{\infty}\left(Q_{3}, T M\right)_{u}^{S_{i n}^{G}(3)}$, then $\mathscr{D}_{A}^{l i n, u}$ satisfies:

$$
\left\langle\mathscr{D}_{A}^{l i n, u} v, w\right\rangle_{L^{2}}=\left\langle v, \mathscr{D}_{A}^{l i n, u} w\right\rangle_{L^{2}}
$$

2. For all $v \in C^{\infty}\left(Q_{4}, T M\right)^{\operatorname{Spin}_{\varepsilon}^{G}(4)}, w \in C^{\infty}\left(Q_{4}, \widehat{T M}^{1}\right)^{\operatorname{Spin}_{\varepsilon}^{G}(4)}$ :

$$
\begin{aligned}
\left\langle\mathscr{D}_{A}^{l i n, u,+} v, w\right\rangle_{L^{2}} & =\left\langle v, \mathscr{D}_{A}^{l i n, u,-} w\right\rangle_{L^{2}}+\int_{X} \operatorname{div}^{\nabla}\left(U_{v, w}\right) * 1 \\
& =\left\langle v, \mathscr{D}_{A}^{l i n, u,+, *} w\right\rangle_{L^{2}}-\int_{\partial X} \pi_{!} g\left(v, c_{4}\left(f_{\overrightarrow{\mathfrak{n}}} \otimes w\right)\right) * 1
\end{aligned}
$$

where $\operatorname{div}^{\nabla}$ denotes the divergence with respect to the $S O(4)$-connection $\nabla$ on $P_{S O(4)}$ to which $A$ projects and $U_{v, w} \in \Gamma(X, T X)$ is defined by $g^{X}\left(U_{v, w}, Z\right)=$ $-\pi_{!}\left(g^{M}\left(v, c_{4}\left(f_{Z} \otimes w\right)\right)\right)$ for $Z \in \Gamma(X, T X)$ and $f_{Z}: Q_{4} \rightarrow \mathbb{R}^{4}$ the corresponding $\operatorname{Spin}_{\varepsilon}^{G}(4)$-equivariant map.

In particular, if $X$ is closed and $A$ projects to the Levi-Civita connection on $X$, or more generally, if $\int_{X} \operatorname{div}^{\nabla}\left(U_{v, w}\right)=0$ for all $v \in C^{\infty}\left(Q_{4}, T M\right)^{\operatorname{Spin}_{\varepsilon}^{G}(4)}, w \in$ $C^{\infty}\left(Q_{4}, \widehat{T M}^{1}\right)^{\operatorname{Spin}_{\varepsilon}^{G}(4)}$, then:

$$
\left\langle\mathscr{D}_{A}^{l i n, u,+} v, w\right\rangle_{L^{2}}=\left\langle v, \mathscr{D}_{A}^{l i n, u,-} w\right\rangle_{L^{2}}
$$

4.5.11 Remark. Note that on the boundary $\partial Y$, the outward pointing normal vector field $\overrightarrow{\mathfrak{n}}$ defines a reduction of the frame bundle $P_{\partial Y}=\left.\left\{\left.f \in P_{S O(3)}\right|_{\partial Y} \mid f\left(e_{1}\right)=\overrightarrow{\mathfrak{n}}\right\} \subset P_{S O(3)}\right|_{\partial Y}$, and a reduction $\left.Q_{\partial Y} \subset Q_{3}\right|_{\partial Y}$. Note that on $Q_{\partial Y}$, we have

$$
g^{M}\left(v, c_{3}\left(f_{\overrightarrow{\mathfrak{n}}} \otimes w\right)\right)=g^{M}\left(v, c_{3}\left(e_{1} \otimes w\right)\right)=g^{M}\left(I_{1} v, w\right)
$$

Similarly, we have a reduction $\left.Q_{\partial X} \subset Q_{4}\right|_{\partial X}$ and on $Q_{\partial X}$ :

$$
g^{M}\left(v, c_{4}\left(f_{\overrightarrow{\mathfrak{n}}} \otimes w\right)\right)=g^{M}\left(v, c_{4}\left(e_{0} \otimes w\right)\right)=g^{M}(v, w)
$$

Proof (of Proposition 4.5.10). Except for allowing torsion connections on $P_{S O(m)}$, the proof resembles the usual proof that the Dirac operator is formally self-adjoint. A similar proof for a Dirac operator obtained from a connection with non-vanishing torsion can be found in [HH06, Thm. 4.5.3], where symplectic Dirac operators are studied.

Consider the covariant derivative $\nabla^{u^{*} T M}$ on $u^{*} T M \rightarrow Q_{3}$, which is the pullback of the Levi-Civita connection on $M$. For $Z \in T Q_{3}$ and $v \in C^{\infty}\left(Q_{3}, T M\right)_{u}^{\operatorname{Spin}_{\varepsilon}^{G}(3) \cong}$ $\Gamma\left(Q_{3}, u^{*} T M\right)^{\operatorname{Spin}_{\varepsilon}^{G}(3)}$ we obtain

$$
\nabla_{Z}^{u^{*} T M} v=\mathcal{K} T v(Z)
$$

Since the Levi-Civita connection is compatible with the metric on $M$, the pullback $\nabla^{u^{*} T M}$ is compatible with the pullback metric on $u^{*} T M$ :

$$
g^{M}\left(\nabla^{u^{*} T M} v, w\right)+g^{M}\left(v, \nabla^{u^{*} T M} w\right)=d\left(g^{M}(v, w)\right) \text { for all } v, w \in C^{\infty}\left(Q_{3}, T M\right)_{u}^{\operatorname{Spin}_{\varepsilon}^{G}(3)} .
$$


Note that if we insert a horizontal lift $\tilde{X} \in T Q$ (with respect to $A$ ) of $X \in T Y$, the right hand side is

$$
d\left(g^{M}(v, w)\right)(\tilde{X})=d_{A}\left(g^{M}(v, w)\right)(\tilde{X})=d \pi_{!}\left(g^{M}(v, w)\right)(X),
$$

where $\pi_{!}\left(g^{M}(v, w)\right) \in C^{\infty}(Y, \mathbb{R})$ is induced by $g^{M}(v, w): Q_{3} \rightarrow \mathbb{R}$, and its exterior derivative on $Y$ is $d \pi_{!}\left(g^{M}(v, w)\right) \in \Omega^{1}(Y, \mathbb{R})$.

Fix a point $p \in Q_{3}, y:=\pi_{Y}(p)$ and let $X_{\ell}:=\pi_{S O}(p)\left(e_{\ell}\right) \in T_{y} Y$ for $\ell \in\{1,2,3\}$. Extend $X_{\ell} \in T_{y} Y$ to vector fields $X_{\ell} \in \Gamma(Y, T Y)$. Since $T Y$ is the associated bundle $T Y=Q_{3} \times_{\operatorname{Spin}_{\varepsilon}^{G}(3)} \mathbb{R}^{3}$, these correspond to $\operatorname{Spin}_{\varepsilon}^{G}(3)$-equivariant maps $f_{\ell}: Q_{3} \rightarrow \mathbb{R}^{3}$. In particular, $X_{\ell}=\pi_{S O}(p)\left(e_{\ell}\right)$ implies that $f_{\ell}(p)=e_{\ell}$. With these choices, we obtain

$$
\begin{aligned}
& g^{M}\left(\mathscr{D}_{A}^{l i n, u}(v)(p), w(p)\right) \\
= & \sum_{\ell=1}^{3} g^{M}\left(c_{3}\left(e_{\ell} \otimes \nabla_{\tilde{X}_{\ell}}^{u^{*} T M} v\right)(p), w(p)\right) \\
= & -\sum_{\ell=1}^{3} g^{M}\left(\nabla_{\tilde{X}_{\ell}}^{u^{*} T M} v(p), c_{3}\left(e_{\ell} \otimes w\right)(p)\right) \\
= & -\sum_{\ell=1}^{3} g^{M}\left(\nabla_{\tilde{X}_{\ell}}^{u^{*} T M} v(p), c_{3}\left(f_{\ell}(p) \otimes w(p)\right)\right) \\
= & -\sum_{\ell=1}^{3} d\left(g^{M}\left(v, c_{3}\left(f_{\ell} \otimes w\right)\right)\right)\left(\left.\tilde{X}_{\ell}\right|_{p}\right)+\sum_{\ell=1}^{3} g^{M}\left(v(p), \nabla_{\tilde{X}_{\ell}}^{u_{\ell}^{*}}\left(c_{3}\left(f_{\ell} \otimes w\right)\right)(p)\right) . \\
= & -\sum_{\ell=1}^{3} d\left(g^{M}\left(v, c_{3}\left(f_{\ell} \otimes w\right)\right)\right)\left(\left.\tilde{X}_{\ell}\right|_{p}\right)+\sum_{\ell=1}^{3} g^{M}\left(v(p), c_{3}\left(\nabla_{\tilde{X}_{\ell}}^{A}\left(f_{\ell}\right) \otimes w\right)(p)\right) \\
& +g^{M}\left(v(p), \mathscr{D}_{A}^{l i n, u}(w)(p)\right) .
\end{aligned}
$$

The first two summand on the right hand side of Equation 4.3 can be interpreted as a divergence:

$$
\begin{aligned}
& -\sum_{\ell=1}^{3} d\left(g^{M}\left(v, c_{3}\left(f_{\ell} \otimes w\right)\right)\right)\left(\tilde{X}_{\ell}\right)+\sum_{\ell=1}^{3} g^{M}\left(v(p), c_{3}\left(\nabla_{\tilde{X}_{\ell}}^{A}\left(f_{\ell}\right) \otimes w\right)(p)\right) \\
& \left.=-\sum_{\ell=1}^{3} d \pi_{!}\left(g^{M}\left(v, c_{3}\left(f_{\ell} \otimes w\right)\right)\right)\left(X_{\ell}\right)-\sum_{\ell=1}^{3} g^{Y}\left(U_{v, w}, \nabla_{X_{\ell}} X_{\ell}\right)\right) \\
& \left.=\sum_{\ell=1}^{3} d\left(g^{Y}\left(U_{v, w}, X_{\ell}\right)\right)\left(X_{\ell}\right)-\sum_{\ell=1}^{3} g^{Y}\left(U_{v, w}, \nabla_{X_{\ell}} X_{\ell}\right)\right) \\
& \left.=\sum_{\ell=1}^{3} g^{Y}\left(\nabla_{X_{\ell}} U_{v, w}, X_{\ell}\right)\right) \\
& =\operatorname{div}^{\nabla}\left(U_{v, w}\right) .
\end{aligned}
$$

We obtain

$$
g^{M}\left(\mathscr{D}_{A}^{l i n, u}(v)(p), w(p)\right)=g^{M}\left(v(p), \mathscr{D}_{A}^{l i n, u}(w)(p)\right)+\operatorname{div}^{\nabla}\left(U_{v, w}\right)(y) .
$$


In particular, integrating over the compact manifold $Y$, we obtain

$$
\left\langle\mathscr{D}_{A}^{l i n, u} v, w\right\rangle_{L^{2}}=\left\langle v, \mathscr{D}_{A}^{l i n, u} w\right\rangle_{L^{2}}+\int_{Y} \operatorname{div}^{\nabla}\left(U_{v, w}\right) * 1 .
$$

Recall that for any vector field $V \in \Gamma(Y, T Y)$, the divergence $\operatorname{div}^{\nabla}(V):=\operatorname{tr}(\nabla V)$ and the divergence with respect to the Levi-Civita connection $\nabla^{L C}$ are related by ${ }^{1}$

$$
\operatorname{div}^{\nabla}(V)=\operatorname{div}^{\nabla^{L C}}(V)+\mathcal{T}^{\nabla}(V)
$$

Finally, we compute using Stokes' theorem:

$$
\begin{aligned}
\int_{Y} \operatorname{div}^{\nabla}\left(U_{v, w}\right) * 1 & =\int_{Y} \operatorname{div}^{\nabla^{L C}}\left(U_{v, w}\right) * 1+\int_{Y} \mathcal{T}^{\nabla}\left(U_{v, w}\right) \\
& =\int_{\partial Y} g^{M}\left(U_{v, w}, \overrightarrow{\mathfrak{n}}\right) * 1+\int_{Y} \mathcal{T}^{\nabla}\left(U_{v, w}\right) \\
& =-\int_{\partial Y} \pi_{!} g^{M}\left(v, c_{3}\left(f_{\overrightarrow{\mathfrak{n}}} \otimes w\right)\right)-\int_{Y} \pi_{!} g^{M}\left(v, c_{3}\left(\mathcal{T}^{\nabla} \otimes w\right)\right)
\end{aligned}
$$

The proof also immediately carries over to the case of $m=4$.

\subsubsection{Dirac operators on manifolds with boundary}

Consider an oriented Riemannian 4-manifold $X$ with boundary $Y=\partial X$ with $\operatorname{Spin}_{\varepsilon}^{G}(4)$ structure $Q_{4} \rightarrow X$. Let $i: Y=\partial X \hookrightarrow X$ the inclusion and $\overrightarrow{\mathfrak{n}} \in \Gamma\left(\partial X, i^{*} T X\right)$ be the outward pointing normal vector field of unit length. We use the induced orientation on $\partial X$, i.e. an orthonormal frame $\left\{v_{1}, v_{2}, v_{3}\right\}$ in $T_{y} \partial X$ is positively oriented if $\left\{\overrightarrow{\mathfrak{n}}, v_{1}, v_{2}, v_{3}\right\}$ is a positively oriented orthonormal frame in $T_{y} X$. Note that $P_{S O(3)}:=\left\{(y, f) \in i^{*} P_{S O(4)} \mid\right.$ $\left.f\left(e_{0}\right)=\left.\overrightarrow{\mathfrak{n}}\right|_{y}\right\}$ is the bundle of oriented orthonormal frames on $\partial X$.

Define $Q_{3}:=\left\{(y, p) \in i^{*} Q_{4}\left|\pi_{S O(4)}(p)\left(e_{0}\right)=\overrightarrow{\mathfrak{n}}\right|_{y}\right\} \stackrel{\jmath}{\hookrightarrow} i^{*} Q_{4}$. This is a principal $\operatorname{Spin}_{\varepsilon}^{G}(3)$ bundle over $\partial X$, where the action is induced by the inclusion $\operatorname{Spin}_{\varepsilon}^{G}(3) \hookrightarrow \operatorname{Spin}_{\varepsilon}^{G}(4)$. This is a $\operatorname{Spin}_{\varepsilon}^{G}(3)$-structure on $\partial X$, the induced $\operatorname{Spin}_{\varepsilon}^{G}(3)$-structure on the boundary.

Given a spinor $u: Q_{4} \rightarrow M$, its restriction $u_{\partial X}:=\left.u\right|_{Q_{3}}: Q_{3} \rightarrow M$ is a spinor on $\partial X$. Given a connection $A \in \mathscr{A}_{4}$ lifting a metric connection $\varphi$ with covariant derivative $\nabla^{X}$,

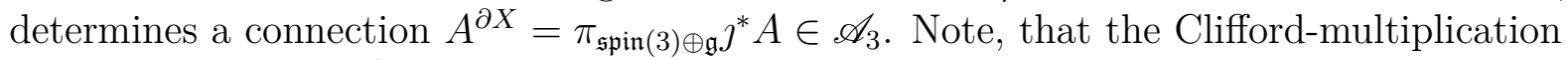
$c_{4}\left(e_{0}\right): T M \rightarrow \widehat{T M}^{1}$ is an isomorphism, which we will use to identify $u_{\partial X}^{*} T M$ and $u_{\partial X}^{*} \widehat{T M}^{1}$. Pushed down to sections of bundles over $\partial X$, this is the Clifford multiplication with the normal vector field $\overrightarrow{\mathfrak{n}}$.

Recall that the second fundamental form

$N \in \Gamma\left(\partial X, T^{*} \partial X \otimes T^{*} \partial X\right) \cong \Gamma\left(Q_{3}, T^{*} Q_{3} \otimes T^{*} Q_{3}\right)_{h o r}^{\operatorname{Spin}_{\varepsilon}^{G}(3)} \cong C^{\infty}\left(Q_{3}, \mathfrak{s p}(1)^{\vee} \otimes \mathfrak{s p}(1)^{\vee}\right)^{\operatorname{Spin}_{\varepsilon}^{G}(3)}$

\footnotetext{
${ }^{1}$ This follows from $g\left(\left(\nabla_{v_{1}}-\nabla_{v_{1}}^{L C}\right) v_{2}, v_{3}\right)=\frac{1}{2}\left(g\left(T^{\nabla}\left(v_{3}, v_{1}\right), v_{2}\right)+g\left(T^{\nabla}\left(v_{3}, v_{2}\right), v_{1}\right)+g\left(T^{\nabla}\left(v_{1}, v_{2}\right), v_{3}\right)\right)$ for any metric-compatible connection $\nabla$, which can be derived using the Koszul formula for the Levi-Civita connection, the metric compatibility of $\nabla$ and $T^{\nabla}\left(v_{1}, v_{2}\right):=\nabla_{v_{1}} v_{2}-\nabla_{v_{2}} v_{1}-\left[v_{1}, v_{2}\right]$.
} 
is defined by $N(v, w):=g\left(\overrightarrow{\mathfrak{n}}, \nabla_{v}^{X} w\right)$ and $\nabla_{v}^{\partial X} w:=\operatorname{pr}_{T \partial X} \nabla_{v}^{X} w$. For $v \in T \partial X$, we can extend $N(v, \cdot) \overrightarrow{\mathfrak{n}}: T \partial X \rightarrow \overrightarrow{\mathfrak{n}} \mathbb{R}$, to a skew-symmetric endomorphism $\left.\left.T X\right|_{\partial X} \rightarrow T X\right|_{\partial X}$. Lifting this to $Q_{3}$, we obtain $\alpha_{\mathfrak{s o}(4)} \in \Omega^{1}\left(Q_{3}, \mathfrak{s o}(4)\right)_{h o r}^{\operatorname{Spin}_{\varepsilon}^{G}(3)}$. Now, define $\alpha:=\nu_{*}^{-1} \alpha_{\mathfrak{s o}(4)} \in$ $\Omega^{1}\left(Q_{3}, \mathfrak{s p i n}(4)\right)_{h o r}^{S p i n_{\varepsilon}^{G}(3)}$, where $\nu_{*}: \mathfrak{s p i n}(4) \rightarrow \mathfrak{s o}(4)$ is the isomorphism induced by the double cover $\operatorname{Spin}(4) \rightarrow S O(4)$. Then $\jmath^{*} A=A^{\partial X}+\alpha$ on $\partial X$. More explicitly, we have $\alpha_{\mathfrak{s o}(4)}(v)=\sum_{\ell=1}^{3} N\left(v, \tilde{e}_{\ell}\right)\left(e_{\ell}^{\vee} \otimes e_{0}-e_{0}^{\vee} \otimes e_{\ell}\right)$, where $v \in T_{p} Q_{3}$, where we interpret $N \in \Gamma\left(Q_{3}, T^{*} Q_{3} \otimes T^{*} Q_{3}\right)_{h o r}^{\operatorname{Spin}_{\varepsilon}^{G}(3)}$ and $\left.\tilde{e}_{\ell}\right|_{p}=\pi_{S O(3)}(p)\left(e_{\ell}\right)$ is any horizontal lift. Therefore, $\alpha(v)=\frac{1}{2} \sum_{\ell=1}^{3} N\left(v, \tilde{e}_{\ell}\right) e_{\ell} e_{0}$.

In the following, we will relate $\left.\left(\mathscr{D}_{A}^{+} u\right)\right|_{Q_{3}}$ and $\mathscr{D}_{A^{\partial X}}\left(u_{\partial X}\right)$, generalizing the results in [KM07, Sec. 4.3-4.5]:

\subsubsection{Lemma.}

In the situation described above, we have

1. $\left.\left(d_{A}^{M} u\right)\right|_{Q_{3}}=d_{A^{\partial X}}^{M} u_{\partial X}+e_{0}^{\vee} \otimes d_{A}^{M} u\left(e_{0}\right)+\alpha \cdot u_{\partial X}$,

2. $\left.\left(\mathscr{D}_{A}^{+} u\right)\right|_{Q_{3}}=c_{4}\left(e_{0}\right)\left(\mathscr{D}_{A^{\partial X}}\left(u_{\partial X}\right)+d_{A}^{M} u\left(\tilde{e}_{0}\right)-\left.\frac{H}{2}\left(\chi_{0}+\chi_{0}^{-}\right)\right|_{u_{\partial X}}\right.$

$$
\left.+\frac{1}{2}\left\langle N_{0},\left.\left(\chi_{2}+\chi_{2}^{-}\right)\right|_{u_{\partial X}}\right\rangle+\frac{1}{2}\left\langle g\left(\overrightarrow{\mathfrak{n}}, * T^{\nabla^{X}}\right),\left.\left(\chi_{A l t}+\chi_{A l t}^{-}\right)\right|_{u_{\partial X}}\right\rangle\right),
$$

where $\alpha \cdot u_{\partial X}:=\left.v_{\alpha_{+}}^{S p(1)_{+}}\right|_{u_{\partial X}}+\left.v_{\alpha_{-}}^{S p(1)_{-}}\right|_{u_{\partial X}}$ with $\alpha_{ \pm}$the $\mathfrak{s p}(1)_{ \pm}$-components of $\alpha, H=\operatorname{tr}(N)$ is the mean curvature, $N_{0}:=\operatorname{pr}_{S_{0}^{2} \mathfrak{s p}(1) \vee} N$ is the traceless symmetric part of the second fundamental form $N$, and $\chi_{0}^{-}, \chi_{2}^{-}, \chi_{\text {Alt }}^{-}$are the vector fields defined in the same way as $\chi_{0}, \chi_{2}, \chi_{A l t}$, but using the $S p(1)_{-}$-action instead of the $S p(1)_{+}$-action.

Proof. The first part immediately follows from $\jmath^{*} A=A^{\partial X}+\alpha$. Applying the Clifford multiplication $c_{4}$, we obtain

$$
\begin{aligned}
\left.\left(\mathscr{D}_{A}^{+} u\right)\right|_{Q_{3}} & =c_{4}\left(d_{A^{\partial X}}^{M}\left(u_{\partial X}\right)+e_{0} \otimes d_{A}^{M} u\left(e_{0}\right)+\alpha \cdot u_{\partial X}\right) \\
& =c_{4}\left(e_{0}\right) c_{3}\left(d_{A^{\partial X}}^{M}\left(u_{\partial X}\right)\right)+c_{4}\left(e_{0}\right) d_{A}^{M} u\left(e_{0}\right)+c_{4}\left(\alpha \cdot u_{\partial X}\right) \\
& =c_{4}\left(e_{0}\right)\left(\mathscr{D}_{A^{\partial X}}\left(u_{\partial X}\right)+d_{A}^{M} u\left(e_{0}\right)+c_{3}\left(\alpha \cdot u_{\partial X}\right)\right) .
\end{aligned}
$$

Since we allow metric connections with torsion, the second fundamental form is no longer symmetric, but

$$
N(v, w)-N(w, v)=g\left(\overrightarrow{\mathfrak{n}}, \nabla_{v}^{X} w-\nabla_{w}^{X} v\right)=g\left(\overrightarrow{\mathfrak{n}}, \nabla_{v}^{X} w-\nabla_{w}^{X} v-[v, w]\right)=g\left(\overrightarrow{\mathfrak{n}}, T^{\nabla^{X}}(v, w)\right) .
$$

Therefore, the skew-symmetric part is $\pi_{\wedge^{2} \mathfrak{s p}(1)^{\vee}} N=\frac{1}{2} g\left(\overrightarrow{\mathfrak{n}}, T^{\nabla^{X}}(v, w)\right)$.

Note that

$$
\begin{aligned}
c_{3}\left(\left.v_{\operatorname{pr}_{\mathfrak{s p}(1)_{+}}}^{S p(1)_{+}}{ }\right|_{u_{\partial X}}\right) & =-\sum_{k=1}^{3} I_{k} v_{\operatorname{pr}_{\mathfrak{s p}(1)_{+}}^{S p(1)_{+}} \alpha\left(e_{k}\right)}||_{u_{\partial X}}=\left.\frac{1}{2} \sum_{k, \ell=1}^{3} N\left(\tilde{e}_{k}, \tilde{e}_{\ell}\right) I_{k} v_{\zeta_{\ell}}^{S p(1)_{+}}\right|_{u_{\partial X}} \\
& =-\left.\frac{H}{2} \chi_{0}\right|_{u_{\partial X}}+\frac{1}{2}\left\langle N_{0},\left.\chi_{2}\right|_{u_{\partial X}}\right\rangle+\frac{1}{2}\left\langle g\left(\overrightarrow{\mathfrak{n}}, * T^{\nabla^{X}}\right),\left.\chi_{A l t}\right|_{u_{\partial X}}\right\rangle .
\end{aligned}
$$

Similarly,

$$
c_{3}\left(\left.v_{\operatorname{pr}_{\mathfrak{s p}(1)_{-}}^{S p(1)_{-}} \alpha}\right|_{u_{\partial X}}\right)=-\left.\frac{H}{2} \chi_{0}^{-}\right|_{u_{\partial X}}+\frac{1}{2}\left\langle N_{0},\left.\chi_{2}^{-}\right|_{u_{\partial X}}\right\rangle+\frac{1}{2}\left\langle g\left(\overrightarrow{\mathfrak{n}}, * T^{\nabla^{X}}\right),\left.\chi_{A l t}^{-}\right|_{u_{\partial X}}\right\rangle .
$$


4.5.13 Example. For $M=\mathbb{H}$ as in Example 2.1.7, and $\nabla^{X}$ the Levi-Civita connection, we have $\chi_{2}=0, \chi_{0}=-\frac{1}{3} \operatorname{tr}(\chi)=\mathrm{id}: \mathbb{H} \rightarrow \mathbb{H}, I_{\ell} v_{\zeta_{\ell}}^{S p(1)}=-\mathrm{id}_{\mathbb{H}}$ and hence

$$
\hat{\alpha}\left(u_{\partial X}\right)=c_{3}\left(\alpha \cdot u_{\partial X}\right)=\frac{1}{2}\left\langle N,\left.\chi\right|_{u_{\partial X}}\right\rangle=-\frac{H}{2} u_{\partial X}
$$

Where $H=\operatorname{tr}(N)$ is the mean curvature of $\partial X$.

\subsection{Examples}

4.6.1 Example (twisted Dirac operator). If $Y$ is an oriented 3-dimensional Riemannian Spin-manifold, $G=\mathbb{Z} / 2 \mathbb{Z} \times O(k)$ with $\varepsilon=(-1,1)$ and $M=S \otimes \mathbb{R}^{k}, P \rightarrow Y$ a principal $O(k)$-bundle with connection $a$ and $A=a+\pi_{S O(3)}^{*} \varphi_{Y} \in \mathscr{A}_{3}$, then we recover the twisted Dirac operator for the bundle $\mathcal{S} \otimes \xi$, where $\xi=P \times_{O(k)} \mathbb{R}^{k}$ :

$$
\mathscr{D}_{A}: \Gamma(\mathcal{S} \otimes \xi) \stackrel{\nabla^{A}}{\longrightarrow} \Gamma\left(T^{*} Y \otimes \mathcal{S} \otimes \xi\right) \stackrel{c_{3} \otimes \mathrm{id}_{\xi}}{\longrightarrow} \Gamma(\mathcal{S} \otimes \xi) .
$$

A similar construction can be done for $m=4$, where we recover

$$
\mathscr{D}_{A}^{+}: \Gamma\left(\mathcal{S}^{+} \otimes \xi\right) \stackrel{\nabla^{A}}{\longrightarrow} \Gamma\left(T^{*} X \otimes \mathcal{S}^{+} \otimes \xi\right) \stackrel{c_{4} \otimes \mathrm{id}_{\xi}}{\longrightarrow} \Gamma\left(\mathcal{S}^{-} \otimes \xi\right) .
$$

If $G=S^{1} \times U(k)$ with $\varepsilon=(-1,-1,1)$ and $M=W \otimes \mathbb{C}^{k}, P \rightarrow Y$ a principal $U(k)$-bundle with connection $a$ and $A=a+\pi_{S O(3)}^{*} \varphi_{Y} \in \mathscr{A}_{3}$, then we recover the Spin ${ }^{\mathrm{c}}$-Dirac operator twisted with the hermitian bundle $E=P \times_{U(k)} \mathbb{C}^{k}$ :

$$
\mathscr{D}_{A}: \Gamma(\mathcal{W} \otimes E) \rightarrow \Gamma(\mathcal{W} \otimes E)
$$

Similarly, in dimension 4, we recover the twisted Dirac operator

$$
\mathscr{D}_{A}^{+}: \Gamma\left(\mathcal{W}^{+} \otimes E\right) \rightarrow \Gamma\left(\mathcal{W}^{-} \otimes E\right)
$$

4.6.2 Example $\left(\boldsymbol{S O}(\boldsymbol{m})\right.$-action). Let $G$ be the trivial group and hence, $\operatorname{Spin}_{\varepsilon}^{G}(m)=$ $S O(m)$. Consider the standard $S O(3)$-action on $\mathbb{H}=\mathbb{R} \oplus \operatorname{Im}(\mathbb{H})$, which we intepret as a hyperkähler manifold $\left(\mathbb{H}, L_{i}, L_{j}, L_{k}\right)$. Then there is a unique $\operatorname{Spin}_{\varepsilon}^{G}(m)$-structure on a three or four dimensional oriented Riemannian manifold, which is just given by the principal $S O(m)$-frame bundle $Q=P_{S O(m)}$ and the only element in $\mathscr{A}$ is the Levi-Civita connection. Recall that the Dirac operator for $\Lambda^{*} \mathbb{R}^{m} \cong C l_{m}$ with the left action of the Clifford algebra on itself is $-\left(d+d^{*}\right)$ (cf. [LM89, Ch. II Thm. 5.12] and use our convention for Clifford multiplication).

1. For $m=3$, we have $\mathbb{H}=\{0\} \oplus \mathbb{H}=C l_{3}^{-} \subset C l_{3}=\mathbb{H} \oplus \mathbb{H}$.

$$
C^{\infty}(Q, \mathbb{H})^{\operatorname{Spin}_{\varepsilon}^{G}(3)} \cong C^{\infty}\left(Q, C l_{3}^{-}\right)^{\operatorname{Spin}_{\varepsilon}^{G}(3)} .
$$

Using the isomorphism $\bigwedge^{0} \mathbb{R} \oplus \bigwedge^{1} \mathbb{R}^{3} \cong C l_{3}^{-},(f, \alpha) \mapsto \frac{1-*}{2} f+\frac{1+*}{2} \alpha$, we can interpret the generalized Dirac operator as $(f, \alpha) \mapsto\left(-d^{*} \alpha,-d f-* d \alpha\right)$. 
2. For $m=4$, we have $\mathbb{H}=C l_{4}^{0,+}=\bigwedge^{0} \mathbb{R}^{4} \oplus \bigwedge_{+}^{2} \mathbb{R}^{4}$ and

$$
C^{\infty}(Q, \mathbb{H})^{S O(4)} \cong \Omega_{+}^{e v}(X) .
$$

A direct computation shows that the generalized Dirac operator is

$$
-\left.\left(d+d^{*}\right)\right|_{\Omega_{+}^{e v}(X)}: \Omega_{+}^{e v}(X) \rightarrow \Omega_{-}^{\text {odd }}(X) .
$$

Using the isomorphisms $\Omega^{0}(X) \oplus \Omega_{+}^{2}(X) \cong \Omega_{+}^{e v}(X),(C, B) \mapsto C+* C+B$ and $\Omega^{1}(X) \cong \Omega_{-}^{\text {odd }}(X), \alpha \mapsto \alpha-* \alpha$, we can identify the generalized Dirac operator with the map

$$
\begin{aligned}
\Omega^{0}(X) \oplus \Omega_{+}^{2}(X) & \rightarrow \Omega^{1}(X), \\
(C, B) & \mapsto d C+d^{*} B .
\end{aligned}
$$

3. For $m=4$, we also have $\mathbb{H} \cong C l_{4}^{1,+} \cong \bigwedge^{1} \mathbb{R}^{4}$, and the corresponding Dirac operator

$$
\begin{aligned}
\Omega^{1}(X) & \rightarrow \Omega_{-}^{e v}(X) \cong \Omega^{0}(X) \oplus \Omega_{-}^{2}(X), \\
\alpha & \mapsto\left(d^{*} \alpha,(d \alpha)_{-}\right) .
\end{aligned}
$$

The difference between two choices in dimension 4 is the rotating action: Once, the rotating action factors through $\operatorname{Spin}(4) \rightarrow S p(1)_{+} \rightarrow S O(3) \curvearrowright \wedge^{0} \mathbb{R}^{4} \oplus \wedge_{+}^{2} \mathbb{R}^{4}$, while in the other case, we have the full $S O(4)$-action on $\wedge^{1} \mathbb{R}^{4}$. These two choices will reappear when we discuss examples of the generalized Seiberg-Witten equations (which uses these Dirac operators). These will lead to the Vafa-Witten equations and the (stable) complex anti-selfduality equations, respectively.

Similarly, we can take a non-trivial group $G$ and take $M=\mathbb{H} \otimes \mathfrak{g}$ with the same permuting $S O(m)$-action and the adjoint action of $G$ on its Lie algebra $\mathfrak{g}$. The Dirac operators for a connection $A$ in a principal $G$-bundle are the same as above, with $d$ and $d^{*}$ replaced by $d_{A}$ and $d_{A}^{*}$, respectively, and all forms taking values in the associated vector bundle for the adjoint action.

4.6.3 Example (Fueter operator). Since we allow the fixed connection on $Y^{3}$ to have torsion, we can use the flat connection induced by a trivialization (a frame) $T Y \cong Y \times \mathbb{R}^{3}$. Note that such a frame always exists for a compact oriented 3-manifold. In the case of a divergence free frame (as considered in [Sal13], for related theories also cf. [HNS09a], [HNS09b]), the Dirac operator is symmetric ([Sal13]) and is also refered to as the Fueter operator. More general Fueter operator (in dimensions 3 and 4, with non-trivial principal bundles) have been studied more recently in [Wal15]. We now give a more detailed description of how the Fueter operator on a 3-manifold with a frame can be understood as a generalized Dirac operator:

Let $Y$ be a compact, orientable 3-manifold. Recall that $Y$ is parallelizable, i.e. we can fix a trivialization $T Y \cong \underline{\mathbb{R}}^{3}$ of the tangent bundle $T Y \rightarrow Y$, given by three nowhere vanishing sections $v_{1}, v_{2}, v_{3}$, which span $T_{y} Y$ at each $y \in Y$. We also denote this frame by $v=\left(v_{1}, v_{2}, v_{3}\right)$. 
Given a frame $v$ on $Y$, we can define a Riemannian metric $g^{v}$ on $Y$ by $g^{v}\left(v_{\ell}, v_{k}\right)=\delta_{\ell, k}$. The frame $v$ is orthonormal in this metric and $\operatorname{vol}_{Y}^{v}:=\alpha_{1} \wedge \alpha_{2} \wedge \alpha_{3} \in \Omega^{3}(Y)$ is a volume form on $Y$ and hence fixes an orientation, where $\alpha_{\ell}:=g^{v}\left(v_{\ell},-\right)$.

The oriented orthonormal frame bundle $P_{S O(3)} \rightarrow Y$ for this metric is the trivial bundle $Y \times S O(3) \rightarrow Y$. Let $Q:=Y \times \operatorname{Spin}_{\varepsilon}^{G}(3) \rightarrow Y$ be the trivial $\operatorname{Spin}_{\varepsilon}^{G}(3)$-bundle. This defines a $\operatorname{Spin}_{\varepsilon}^{G}(3)$-structure on $Y$.

First, note that the space of spinors is $\mathscr{N}_{3}=C^{\infty}(Y, M)$. Furthermore, we have two connections from the previous constructions: Instead of using the Levi-Civita connection for the metric $g^{v}$, we chose the flat connection (with torsion) $\nabla^{\text {flat }}=\left(v^{-1}\right)^{*} \nabla^{\mathbb{R}^{3}}$ pulled back from $\underline{\mathbb{R}}^{3}$ to $T Y$ via the frame $v$. Note that by construction $\nabla^{\text {flat }} g^{v}=0$. However, its torsion does not vanish in general:

$$
T^{\nabla^{f l a t}}\left(v_{\ell}, v_{k}\right)=v\left(d\left(v^{-1}\left(v_{k}\right)\right)\left(v_{\ell}\right)\right)-v\left(d\left(v^{-1}\left(v_{\ell}\right)\right)\left(v_{k}\right)\right)-\left[v_{\ell}, v_{k}\right]=-\left[v_{\ell}, v_{k}\right]
$$

and therefore the torsion 1-form is $\mathcal{T}^{\text {flat }}\left(v_{k}\right)=\sum_{\ell} g^{v}\left(v_{\ell},\left[v_{k}, v_{\ell}\right]\right)$.

The corresponding generalized Dirac operator is

$$
\mathscr{D}_{A} u=\sum_{\ell=1}^{3} c_{3}\left(v_{\ell} \otimes d u\left(v_{\ell}\right)\right)=-\sum_{\ell=1}^{3} I_{\ell} d u\left(v_{\ell}\right) \in C^{\infty}(Y, T M)_{u}
$$

for a spinor $u \in C^{\infty}(Y, M)$. Up to a sign, this is the Fueter-operator studied in [HNS09a], [HNS09b], [Sal13].

In [Sal13], this operator is considered in the situation where another volume form $\operatorname{vol}_{Y}$ is fixed and $v$ is a divergence-free positive frame with respect to this volume form, i.e. $\mathcal{L}_{v_{\ell}} \operatorname{vol}_{Y}=0$ and $\operatorname{vol}_{Y}\left(v_{1}, v_{2}, v_{3}\right)>0$. With $h:=\operatorname{vol}_{Y}\left(v_{1}, v_{2}, v_{3}\right) \in C^{\infty}\left(Y, \mathbb{R}_{>0}\right)$, we have $\operatorname{vol}_{Y}=h \operatorname{vol}_{Y}^{v}=\operatorname{vol}_{Y}^{g}$ is the volume form associated to the metric $g:=h^{\frac{2}{3}} g^{v}$.

Note that in particular, we have $\operatorname{div}^{\nabla^{L C, g}}\left(v_{\ell}\right)=\mathcal{L}_{v_{\ell}} v o l_{Y}^{g}=0$, where $\nabla^{L C, g}$ is the Levi-Civita connection for the metric $g=h^{\frac{2}{3}} g^{v}$. Furthermore,

$$
v_{\ell}(h)=\mathcal{L}_{v_{\ell}}(h)=\mathcal{L}_{v_{\ell}}\left(\operatorname{vol}_{Y}^{g}\left(v_{1}, v_{2}, v_{3}\right)\right)=\operatorname{div}^{\nabla^{L C, g}}\left(v_{\ell}\right) h+\sum_{k} g^{v}\left(\left[v_{\ell}, v_{k}\right], v_{k}\right) h
$$

If $Y$ is closed, we can varify that the linearized Dirac operator is formally self-adjoint:

Indeed, using

$$
\begin{aligned}
& \operatorname{div}^{\operatorname{vol}_{Y}}(U) \operatorname{vol}_{Y}=\mathcal{L}_{U}\left(h v o l_{Y}^{v}\right)=h \mathcal{L}_{U} \operatorname{vol}_{Y}^{v}+U(h) \operatorname{vol}_{Y}^{v}=h \operatorname{div}^{v^{v}} l_{Y}^{v}(U) \operatorname{vol}_{Y}^{v}+U(h) v o l_{Y}^{v} \\
& =\left(\operatorname{div}^{v^{v}} l_{Y}^{v}(U)+U(\ln (h))\right) \operatorname{vol}_{Y}
\end{aligned}
$$


for any vector fields $U \in \Gamma(Y, T Y)$, we can compute for $U=\sum_{\ell} f_{\ell} v_{\ell}$ with $f_{\ell}:=g^{v}\left(U, v_{\ell}\right)$ :

$$
\begin{aligned}
\operatorname{div}^{\nabla^{\text {flat }}}(U) & =\operatorname{div}^{\nabla^{L C, g}}(U)+\mathcal{T}^{\nabla^{\text {flat }}}(U)=\operatorname{div}^{\nabla^{L C, g}}(U)-U(\ln (h))+\mathcal{T}^{\nabla^{\text {flat }}}(U) \\
& =\operatorname{div}^{\nabla^{L C, g}}(U)-h^{-1} U(h)+\sum_{\ell} f_{\ell} \mathcal{T}^{\nabla^{\text {flat }}}\left(v_{\ell}\right) \\
& =\operatorname{div}^{\nabla^{L C, g}}(U)-\sum_{\ell} h^{-1} f_{\ell} v_{\ell}(h)+\sum_{k, \ell} f_{\ell} g^{v}\left(v_{k},\left[v_{\ell}, v_{k}\right]\right) \\
& =\operatorname{div}^{\nabla^{L C, g}}(U)-\sum_{\ell} f_{\ell} \operatorname{div}^{\nabla^{L C, g}}\left(v_{\ell}\right)-\sum_{k, \ell} f_{\ell} g^{v}\left(v_{k},\left[v_{\ell}, v_{k}\right]\right)+\sum_{k, \ell} f_{\ell} g^{v}\left(v_{k},\left[v_{\ell}, v_{k}\right]\right) \\
& =\operatorname{div}^{\nabla^{L C, g}}(U) .
\end{aligned}
$$

In particular, $\int_{Y} \operatorname{div}^{\nabla^{\text {flat }}}\left(U_{v, w}\right) * 1=\int_{Y} \operatorname{div}^{\nabla^{\text {flat }}}\left(U_{v, w}\right) * 1=0$ and the formal self-adjointness follows from Proposition 4.5.10.

4.6.4 Remark. Note that instead of using a trivial principal $G$-bundle, we could have used a non-trivial principal $G$-bundle as well. 



\section{Chapter 5}

\section{The Seiberg-Witten equations}

In this chapter, we expain the Seiberg-Witten equations associated to a hyperkähler manifold $M$ with permuting $\operatorname{Spin}_{\varepsilon}^{G}(m)$-action for $m \in\{3,4\}$ and give an overview over various examples of these equations that have been studied in the literature.

For this purpose, we fix a compact Lie group $G$, an central element $\varepsilon \in Z(M)$ satisfying $\varepsilon^{2}=1$, a $\operatorname{Spin}_{\varepsilon}^{G}(3)$-structure $Q_{3} \rightarrow P_{S O(3)} \times_{Y} P_{G / \varepsilon}$ on a 3-dimensional compact oriented Riemannian manifold $Y$ and a $\operatorname{Spin}_{\varepsilon}^{G}(4)$-structure $Q_{4} \rightarrow P_{S O(4)} \times_{X} P_{G / \varepsilon}$ on a 4-dimensional compact oriented Riemannian manifold $X$. To write the Seiberg-Witten equations, we also fix an $A d$-invariant scalar product $\langle\cdot, \cdot\rangle_{\mathfrak{g}}$ on the Lie algebra $\mathfrak{g}$. We use this to identify $\mathfrak{g} \cong \mathfrak{g}^{\vee}$. Finally, let $\mu: M \rightarrow \mathfrak{g}^{\vee} \otimes \mathfrak{s p}(1)^{\vee}$ be the $\operatorname{Spin}_{\varepsilon}^{G}(m)$-equivariant hyperkähler moment map for the $G$-action (constructed explicitly in [Pid04, Sec. 2.2.1], also see Proposition 2.2.7). Another survey on these equations can be found in [Hay15a].

\subsection{Seiberg-Witten equations}

We have now collected all the necessary ingrediants to write the generalized Seiberg-Witten equations in dimensions three and four.

5.1.1 Definition. For $(u, A) \in \mathscr{C}_{3}=\mathscr{N}_{3} \times \mathscr{A}_{3}$, consider the generalized Seiberg-Witten equations in three dimensions, which were first studied in [Tau99]:

$$
\left\{\begin{array}{l}
\mathscr{D}_{A}(u)=0 \\
* F_{a}+\Phi_{3}(u)=0
\end{array}\right.
$$

where $a$ is the $\mathfrak{g}$-component of $A \in \mathscr{A}_{3}$, the Hodge star operator $*: \wedge^{2}\left(\mathbb{R}^{3}\right)^{\vee} \rightarrow\left(\mathbb{R}^{3}\right)^{\vee}$ induces $*: \Omega^{2}\left(Q_{3}, \mathfrak{g}\right)_{h o r}^{\operatorname{Spin}_{\varepsilon}^{G}(3)} \rightarrow \Omega^{1}\left(Q_{3}, \mathfrak{g}\right)_{h o r}^{\operatorname{Spin}_{\varepsilon}^{G}(3)}$ and the moment map defines $\Phi_{3}(u) \in$ $\Omega^{1}\left(Q_{3}, \mathfrak{g}\right)_{h o r}^{\operatorname{Spin}_{\varepsilon}^{G}(3)} \cong C^{\infty}\left(Q_{3}, \mathfrak{g} \otimes\left(\mathbb{R}^{3}\right)^{\vee}\right)^{\operatorname{Spin}_{\varepsilon}^{G}(3)}$ as the composition

$$
Q_{3} \stackrel{u}{\rightarrow} M \stackrel{\mu}{\rightarrow} \mathfrak{g}^{\vee} \otimes \mathfrak{s p}(1)^{\vee} \cong \mathfrak{g} \otimes\left(\mathbb{R}^{3}\right)^{\vee}
$$


5.1.2 Definition. For $(u, A) \in \mathscr{C}_{4}=\mathscr{N}_{4} \times \mathscr{A}_{4}$, consider the generalized Seiberg-Witten equations in four dimensions, which were first studied in [Pid04]:

$$
\left\{\begin{array}{l}
\mathscr{D}_{A}(u)=0 \\
F_{a}^{+}+\Phi_{4}(u)=0
\end{array}\right.
$$

where $a$ is the $\mathfrak{g}$-component of $A \in \mathscr{A}_{4}, F_{a}^{+} \in \Omega_{+}^{2}\left(Q_{4}, \mathfrak{g}\right)_{h o r}^{\operatorname{Spin}_{\varepsilon}^{G}(3)}$ is the selfdual part of the curvature $F_{a}$ of $a$, and $\Phi_{4}(u) \in \Omega_{+}^{2}\left(Q_{4}, \mathfrak{g}\right)^{\operatorname{Spin}_{\varepsilon}^{G}(4)} \cong C^{\infty}\left(Q_{4}, \mathfrak{g} \otimes\left(\bigwedge_{+}^{2} \mathbb{R}^{4}\right)^{\vee}\right)^{\operatorname{Spin}_{\varepsilon}^{G}(4)}$ is defined as the composition

$$
Q_{4} \stackrel{u}{\rightarrow} M \stackrel{\mu}{\rightarrow} \mathfrak{g}^{\vee} \otimes \mathfrak{s p}(1)^{\vee} \cong \mathfrak{g} \otimes \bigwedge_{+}^{2}\left(\mathbb{R}^{4}\right)^{\vee}
$$

In both cases, we obtain a moduli space $\mathcal{M}$, i.e. the quotient of the space of solutions by the action of the gauge group $\mathscr{G}_{m}$.

5.1.3 Note. Note that the left hand side of the generalized Seiberg-Witten equations is a section in an (infinite-dimensional) vector bundle over the configuration space. Details on this point of view can be found in [Cal10, Ch. 4].

\section{$5.2 \quad$ Examples}

Here is a list of examples of the generalized Seiberg-Witten equations. By default, we use the Levi-Civita connection as the fixed connection on the base manifold.

\section{Anti-selfduality equation}

- $G$ Lie group, $\varepsilon=1$,

- $\operatorname{Spin}_{\varepsilon}^{G}(m)=S O(m) \times G, \operatorname{Spin}_{\varepsilon}^{G}(m)$-structure: principal $G$-bundle $P \rightarrow Z$,

- $M=\{*\}$,

- $\mathscr{N}_{m}=\{*\}, \mathscr{A}_{m}=\mathscr{A}(P \rightarrow Z)$,

- 3D equations: $F_{a}=0, \mathcal{M}=\mathcal{M}_{\text {flat }}(P)$,

- 4D equations: $F_{a}^{+}=0$ (anti-selfduality equation), $\mathcal{M}=\mathcal{M}_{\text {asd }}(P)$.

By allowing the hyperkähler manifold to be just one point $M=\{*\}$, the equations reduce to $F_{a}^{+}=0$ in four dimensions and the $F_{a}=0$ in the three-dimensional case. The solutions are the anti-selfdual connection in four dimensions and flat connections in three dimensions. The moduli space of the anti-selfduality equations was used by Donaldson to study smooth 4-dimensional topology (starting with [Don83]), which turned out to be very fruitful and lead to the Donaldson polynomials, which are invariants of smooth structures on 4-manifolds and later to Floer homology [Flo88] (also cf. [Don02]). 


\section{Seiberg-Witten equations}

- $G=S^{1}, \varepsilon=-1$,

- $\operatorname{Spin}_{\varepsilon}^{G}(m)=\operatorname{Spin}^{c}(m), \operatorname{Spin}_{\varepsilon}^{G}(m)$-structure: $\operatorname{Spin}^{c}(m)$-structure,

- $M=\mathbb{H}$ as $\operatorname{Spin}^{c}(3)$-representation $W$ or $\operatorname{Spin}^{c}(4)$-representation $W^{+}$,

- $\mathscr{N}_{3}=\Gamma(Y, \mathcal{W}), \mathscr{N}_{4}=\Gamma\left(X, \mathcal{W}^{+}\right), \mathscr{A}_{m}=\mathscr{A}\left(P_{\text {det }} \rightarrow Z\right)$,

- 3D equations: 3D Seiberg-Witten equations,

- 4D equations: 4D Seiberg-Witten equations.

Seiberg-Witten equations first appeared in [SW94]. Consider $M=\mathbb{H}$ as in Example 2.2.6, $G=S^{1}$ and $\varepsilon=-1$. In this case, a $\operatorname{Spin}_{-1}^{S^{1}}(m)$-structure is the same as a $\operatorname{Spin}^{c}(m)$ structure and the Dirac operator is the usual $\operatorname{Spin}^{c}(m)$ Dirac operator.

Note that in the literature, the most common form of the Seiberg-Witten equations is to apply Clifford multiplication to the second equation and, thus, get an equation for skew-hermitian endomorphisms of the spinor bundle (cf. [KM07]). The second equation then reads $c_{3}\left(F_{a}\right)=\left(u \otimes u^{*}\right)_{0}$ in dimension three and $c_{4}\left(F_{a}^{+}\right)=\left(u \otimes u^{*}\right)_{0}$ in dimension four.

The Seiberg-Witten equations turned out to be a very useful tool in 4-dimensional smooth topology and many results that had been proved using the anti-selfdualty equation and Donaldson theory, were reproved in a simpler way using the Seiberg-Witten equations. Floer homology groups have been defined in this case in [KM07].

\section{Harmonic spinors}

- $G=\mathbb{Z} / 2 \mathbb{Z}, \varepsilon=-1$,

- $\operatorname{Spin}_{\varepsilon}^{G}(m)=\operatorname{Spin}(m), \operatorname{Spin}_{\varepsilon}^{G}(m)$-structure: $\operatorname{Spin}(m)$-structure,

- $M=\mathbb{H}$ as $\operatorname{Spin}(3)$-representation $S$ or $\operatorname{Spin}(4)$-representation $S^{+}$,

- $\mathscr{N}_{3}=\Gamma(Y, \mathcal{S}), \mathscr{N}_{4}=\Gamma\left(X, \mathcal{S}^{+}\right), \mathscr{A}_{m}=\{*\}$,

- 3D equations: $\mathscr{D}_{A} u=0$,

- 4D equations: $\mathscr{D}_{A}^{+} u=0$.

Choosing $G=\mathbb{Z} / 2 \mathbb{Z}$ and the usual $\operatorname{Spin}(m)$-representation $\mathbb{H}$, solutions of the generalized Seiberg-Witten equations are harmonic spinors. For $X=\mathbb{R}^{4} \cong \mathbb{H}$, we recover the equation studied by Fueter [Fue34]. For this reason, the generalized Dirac operator is sometimes called Fueter operator. 


\section{Fueter operator from a frame}

- $G=\mathbb{Z} / 2 \mathbb{Z}, \varepsilon=-1$,

- 3-manifold $Y$ with frame $v: \underline{\mathbb{R}}^{3} \cong T Y$, and the flat connection (with torsion) $\nabla^{\text {flat }}=\left(v^{-1}\right)^{*} \nabla^{\mathbb{R}^{3}}$ pulled back from $\underline{\mathbb{R}}^{3}$ to $T Y$ via the frame $v$,

- trivial $\operatorname{Spin}(3)$-structure induced by the frame,

- $M$ hyperkähler manifold,

- $\mathscr{N}_{3}=C^{\infty}(Y, M)$,

- 3D equations: $\mathscr{D}_{A} u=0$.

As we have seen in Example 4.6.3, this recovers the Fueter operator studied in [Sal13], [HNS09a], [HNS09b] and the corresponding Hyperkähler Floer theory. Allowing nontrivial bundles and different connections leads to the Fueter operators in dimensions 3 and 4 studied in [Wal15].

\section{Vafa-Witten equations}

- $G$ compact Lie group, $\varepsilon=1$,

- $\operatorname{Spin}_{\varepsilon}^{G}(m)=S O(m) \times G, \operatorname{Spin}_{\varepsilon}^{G}(m)$-structure: principal $G$-bundle $P \rightarrow Z$,

- $M=\mathbb{H} \otimes \mathfrak{g}$ as $S O(3) \times G$-representation, $\left(\wedge^{0} \oplus \Lambda^{1}\right) \otimes \mathfrak{g}$ or $S O(4) \times G$-representation, $\left(\wedge^{0} \oplus \Lambda_{+}^{2}\right) \otimes \mathfrak{g}$

- $\mathscr{N}_{3}=\Omega^{0}\left(Y, \mathfrak{g}_{P}\right) \oplus \Omega^{1}\left(Y, \mathfrak{g}_{P}\right), \mathscr{N}_{4}=\Omega^{0}\left(X, \mathfrak{g}_{P}\right) \oplus \Omega_{+}^{2}\left(X, \mathfrak{g}_{P}\right), \mathscr{A}_{m}=\mathscr{A}(P \rightarrow Z)$,

- 3D equations: 3D Vafa-Witten equations,

- 4D equations: 4D Vafa-Witten equations ([VW94]).

Consider a compact Lie group $G$ with an $A d$-invariant scalar product $\langle\cdot, \cdot\rangle_{\mathfrak{g}}$ on its Lie algebra $\mathfrak{g}$. Let $M:=\mathbb{H} \otimes \mathfrak{g}$ with the action of $\operatorname{Spin}_{1}^{G}(m)=S O(m) \times G$ given by the action $S O(m) \curvearrowright \mathbb{H}$ and the adjoint action of $G$ on its Lie algebra $\mathfrak{g}$. Then $M$ has a natural hyperkähler structure induced by the hyperkähler structure on $\mathbb{H}$ given in Example 2.1.7. A $\operatorname{Spin}_{1}^{G}(m)$-structure $Q$ on a manifold $Z(m=\operatorname{dim}(Z) \in\{3,4\})$ has a corresponding principal $G$-bundle $P \rightarrow Z$, whose isomorphism class determines the $\operatorname{Spin}_{1}^{G}(m)$-structure uniquely. Using the Levi-Civita connection $\varphi$ on $P_{S O(m)}$, we obtain $\mathscr{A} \cong \mathscr{A}(P \rightarrow Z)$.

The moment map for the $G$-action on $\mathbb{H} \otimes \mathfrak{g}$ is well-known from the ADHM-construction (which, however, has a different $S p(1)$-action). Its components are

$$
\begin{aligned}
& \mu_{1}(T)=-\left[T_{0}, T_{1}\right]-\left[T_{2}, T_{3}\right], \\
& \mu_{2}(T)=-\left[T_{0}, T_{2}\right]-\left[T_{3}, T_{1}\right], \\
& \mu_{3}(T)=-\left[T_{0}, T_{3}\right]-\left[T_{1}, T_{2}\right],
\end{aligned}
$$


where $T=T_{0}+i T_{1}+j T_{2}+k T_{3}$. The full moment map is given by $\mu(T)=-\left[T_{0}, T_{+}\right]-$ $\llbracket T_{+}, T_{+} \rrbracket \in \mathfrak{s p}(1) \otimes \mathfrak{g}$, where $T_{+}=i T_{1}+j T_{2}+k T_{3}$ and $\llbracket \cdot, \cdot \rrbracket$ the following bracket on $\mathfrak{g} \otimes \operatorname{Im}(\mathbb{H}): \llbracket h \otimes X, h^{\prime} \otimes X^{\prime} \rrbracket:=\frac{1}{4}\left[h, h^{\prime}\right] \otimes\left[X, X^{\prime}\right] \in \operatorname{Im}(\mathbb{H}) \otimes \mathfrak{g}$ for $X, X^{\prime} \in \mathfrak{g}$ and $h, h^{\prime} \in \operatorname{Im}(\mathbb{H})$.

1. $(m=4)$ Let $X=Z$ be a Riemannian 4-manifold. Using the isomorphisms $C^{\infty}\left(Q, \bigwedge_{+}^{e v}\left(\mathbb{R}^{4}\right)^{\vee}\right)^{S p i n} 1_{1}^{G}(4) \cong \Omega^{0}\left(X, \mathfrak{g}_{P}\right) \oplus \Omega_{+}^{2}\left(X, \mathfrak{g}_{P}\right)$ and $\widehat{T M}^{1}=\bigwedge_{-}^{\text {odd }}\left(\mathbb{R}^{4}\right)^{\vee} \otimes \mathfrak{g}$ we obtain $C^{\infty}\left(Q, T \hat{M}^{1}\right)^{\operatorname{Spin}_{1}^{G}(4)}=\Omega_{-}^{\text {odd }}\left(X, \mathfrak{g}_{P}\right) \cong \Omega^{1}\left(X, \mathfrak{g}_{P}\right)$ from Example 4.6.2. Using these identifications, the Dirac operator $\mathscr{D}_{A}$ on a generalized spinor $(C, B) \in$ $\Omega^{0}\left(X, \mathfrak{g}_{P}\right) \oplus \Omega_{+}^{2}\left(X, \mathfrak{g}_{P}\right)$ is $d_{A} C+d_{A}^{*} B \in \Omega^{1}\left(X, \mathfrak{g}_{P}\right)$, where $A \in \mathscr{A}(P \rightarrow X) \cong \mathscr{A}$. Thus the first equation is $d_{A} C+d_{A}^{*} B=0$.

The (4-dimensional) generalized Seiberg-Witten equations thus give the Vafa-Witten equations [VW94] for $A \in \mathscr{A}(P \rightarrow X), B \in \Omega_{+}^{2}\left(X, \mathfrak{g}_{P}\right)$ and $C \in \Omega^{0}\left(X, \mathfrak{g}_{P}\right)$ :

$$
\begin{aligned}
d_{A} C+d_{A}^{*} B & =0 \\
F_{A}^{+}-[C, B]-\llbracket B, B \rrbracket & =0
\end{aligned}
$$

2. $(m=3)$ Let $Y=Z$ be a Riemannian 3-manifold. From Example 4.6.2 we know that $C^{\infty}(Q, \mathbb{H})^{\operatorname{Spin}_{\varepsilon}^{G}(3)} \cong \Omega^{0}\left(Y, \mathfrak{g}_{P}\right) \oplus \Omega^{1}\left(Y, \mathfrak{g}_{P}\right)$ and the generalized Dirac operator is given by

$$
\begin{aligned}
\mathscr{D}_{A}: \Omega^{0}\left(Y, \mathfrak{g}_{P}\right) \oplus \Omega^{1}\left(Y, \mathfrak{g}_{P}\right) & \rightarrow \Omega^{0}\left(Y, \mathfrak{g}_{P}\right) \oplus \Omega^{1}\left(Y, \mathfrak{g}_{P}\right), \\
(C, B) & \mapsto\left(-d_{A}^{*} B,-d_{A} C-* d_{A} B\right)
\end{aligned}
$$

The (3-dimensional) generalized Seiberg-Witten equations thus give the following equations for $A \in \mathscr{A}(P \rightarrow Y), B \in \Omega^{1}\left(Y, \mathfrak{g}_{P}\right)$ and $C \in \Omega^{0}\left(Y, \mathfrak{g}_{P}\right)$ :

$$
\begin{aligned}
d_{A}^{*} B & =0 \\
d_{A} C+* d_{A} B & =0 \\
* F_{A}-[C, B]-\llbracket B, B \rrbracket & =0
\end{aligned}
$$

\section{Complex anti-selfduality equations for $G^{c}$}

- $G$ compact Lie group, $\varepsilon=1$,

- $\operatorname{Spin}_{\varepsilon}^{G}(4)=S O(4) \times G, \operatorname{Spin}_{\varepsilon}^{G}(4)$-structure: principal $G$-bundle $P \rightarrow Z$,

- $M=\mathbb{H} \otimes \mathfrak{g}$ as $S O(4) \times G$-representation $\wedge^{1} \otimes \mathfrak{g}$,

- $\mathscr{N}_{4}=\Omega^{1}\left(X, \mathfrak{g}_{P}\right), \mathscr{A}_{m}=\mathscr{A}(P \rightarrow Z)$,

- 4D equations: (stable) complex anti-selfduality equations. 
Let $B=a+i b \in \mathscr{A}\left(P^{c}\right)$, with $a \in \mathscr{A}(P \rightarrow Z)$ and $b \in \Omega^{1}\left(X, \mathfrak{g}_{P}\right)$, where $P^{c}:=P \times_{G} G^{c}$ with $G^{c}$ the complexified Lie group. Then the generalized Seiberg-Witten equations can be written as

$$
\begin{aligned}
& d_{a}^{*} b=0 \\
& F_{B}^{+}=0 .
\end{aligned}
$$

The three-dimensional analogue of these equations agrees with the three-dimensional Vafa-Witten equations.

These equations for $G^{c}=S L_{2}(\mathbb{C})$ have recently been studied by Taubes in [Tau13b], [Tau13a], [Tau14], who proved a generalization of Uhlenbeck's compactness theorem in this case. The interpretation of these equations as generalized Seiberg-Witten equations is also discussed in [Hay15a].

\section{$\operatorname{Pin}^{-}(2)$-monopole equations}

- $G=\operatorname{Pin}^{-}(2)=S^{1} \cup j S^{1} \subset S p(1), \varepsilon=-1$,

- $\operatorname{Spin}_{\varepsilon}^{G}(m)=\operatorname{Spin}_{\varepsilon}^{\operatorname{Pin}^{-}(2)}(m)$,

- $M=\mathbb{H}$ as $\operatorname{Spin}_{-1}^{\operatorname{Pin}^{-}(2)}(m)$-representation, where $\operatorname{Pin}^{-}(2) \subset S p(1)$ acts hyperkähler,

- 3D equations: Pin $^{-}(2)$-monopole equations,

- 4D equations: Pin $^{-}(2)$-monopole equations.

Nakamura ([Nak13]) uses the generalized Seiberg-Witten equations for $G=\operatorname{Pin}^{-}(2)=$ $S^{1} \cup j S^{1} \subset S p(1)$ and $M=\mathbb{H}$ with the $\operatorname{Pin}^{-}(2)$-action $(g, h) \mapsto h g^{-1}$ to study intersection forms with local coefficients on 4-manifolds. The $\mathrm{Pin}^{-}(2)$-monopole equations are also used by Manolescu ([Man16]) to disprove the Triangulation Conjecture in dimensions $\geq 5$.

Linear actions $G \rightarrow S p(n) \curvearrowright \mathbb{H}^{n}$

Similar to $G=S^{1}$ for the Seiberg-Witten equations, $G=U(n)$ for the $U(n)$-monopole equations, $G=\operatorname{Pin}(2)$ for the $\operatorname{Pin}(2)$-monopole equations, we can also take other subgroups $G \rightarrow S p(n) \curvearrowright \mathbb{H}^{n}$, with the moment map from Example 2.1.23.

\section{Hyperkähler quotients}

Another possibility is to consider a hyperkähler quotient of a manifold $M$ with permuting action by a Lie group $H$, and, if this admits a permuting action, study the generalized Seiberg-Witten equations with values in this quotient $M_{0}$. Using [Hay12, Thm. 4.6], which was independently discovered by Pidstrygach, solutions to the generalized Seiberg-Witten equations with values in the hyperkähler quotient correspond to solutions of a similar set of equations for a connection and a spinor with values in $M$. 
Examples for $M_{0}$ include the moduli space of of framed $S U(n)$-instantons of charge $k$ on $\mathbb{R}^{4}$ obtained using the ADHM construction or infinite-dimensional examples including the moduli space of framed $G$-instantons on $\mathbb{R}^{4}$ obtained as a hyperkähler reduction of the space of connection, as well as some moduli spaces of solutions to Nahm's equations (including the moduli space of Bogomolny monopoles).

\section{$\operatorname{Spin}(7)$-instantons and instanton-valued spinors}

In [Hay12], Haydys proves that the generalized Seiberg-Witten equations in dimension 4 with values in a suitable space of connections $\mathscr{A}^{0}$ on a principal bundle $P \rightarrow \mathbb{R}^{4}$ (with framing at infinity) are (up to a order zero term) the Spin(7)-instanton equations on the total space of a spinor-bundle over the 4-manifold. These are also closely related to harmonic spinors with values in the moduli space of framed instantons.

\section{Relatives of the $U(n)$-monopole equations}

- $G=U(n)$,

- $E \rightarrow X$ a $r k(E)=n$ hermitian vector bundle with corresponding principal $U(n)$ bundle $P \rightarrow X$,

- $\operatorname{Spin}_{-1}^{U(n)}(m)=(\operatorname{Spin}(m) \times U(n)) / \pm 1$,

- $M=S^{(+)} \otimes_{\mathbb{C}} \mathbb{C}^{n}$, where $\mathbb{C}^{n}$ is the tautological representation of $U(n)$,

- equations: $U(n)$-monopole equations.

Note that $U(n) /(\mathbb{Z} / n \mathbb{Z})=S^{1} \times P U(n)$ and hence in particular, for $n=2, \mathfrak{u}(2) \cong$ $i \mathbb{R} \oplus \mathfrak{s o}(3)$. Therefore, the second equation splits into an equation involving the curvature of the determinant line bundle and an equation involving the $P U(2) \cong S O(3)$-connection.

5.2.1 Remark. There are several (elliptic) systems of equations closely related to these:

1. It is possible to study the full generalized Seiberg-Witten equations for $G=U(2)$, even though the second equation splits. For example, these are discussed in [Zen12].

2. Pidstrygach and Tyurin [PT95] studied the case of a $P U(2)$-bundle $\xi$ with fixed lift to a $U(2)$-bundle $E$. Their equations are closely related to the $U(2)$-monopole equations. These are: the first (Dirac equation), the projection of the second equation to $\mathfrak{s u}(2)$ and the condition that the curvature of the determinant bundle is a fixed 2-form $\omega \in \Omega^{2}(X, i \mathbb{R}): F_{a_{d e t}}=\omega$ ( $\omega$ in certain cohomology class, $\omega$ is related to a perturbation).

3. In contrast, Teleman [Tel00] (also in previous collaborations with Ch. Okonek) fixes the connection on the determinant line bundle and writes the projection of the generalized Seiberg-Witten equations for $G=U(2)$ to $\mathfrak{s u}(2)$, where he only considers connections which induce the fixed connection on the determinant line bundle. 
4. Feehan and Leness ([FL98], [FL01]) also fix a unitary connection on the square root of the determinant line bundle of $W^{+}$. These are generalized Seiberg-Witten equations for $G=S U(2)$.

These appear in the context of the $S O(3)$-monopole program ([PT95]). The idea for the proof of the equivalence of the Donaldson polynomial and the Seiberg-Witten invariants is the look at the fixed points of the $S^{1}$-action on the moduli space of $P U(2)$-monopoles. These are the $P U(2)$-instantons and $U(1)$-monopoles (for a rank 1 subbundle of $\mathcal{S}^{+} \otimes E$ ). Quotienting the moduli space of $P U(2)$-monopoles, one obtains a cobordism between a projective bundle over the moduli spaces of $P U(2)$-instantons and projective bundles over the moduli spaces of $U(1)$-monopoles. 


\section{Chapter 6}

\section{Lichnerowicz-Weitzenböck formulae}

In this chapter we present 3-dimensional versions of the 4-dimensional LichnerowiczWeitzenböck formulae in [Sch10] and [Pid04]. Note that our conventions differ in some minor details from the conventions in [Sch10](the symplectic forms and the moment map differ by a sign) and also from those used in [Pid04](in particular we use the other Clifford module structure on TM, cf. [Cal10][Note 3.5.2, Section 2.3.2]). A LichnerowiczWeitzenböck formula for 3-dimensional generalized Dirac operator first appeared in [Tau99].

Before proving the Lichnerowicz-Weitzenböck formulae, we first study the different Dirac Laplacians appearing in the Lichnerowicz-Weitzenböck formulae, and how they are related to each other (Proposition 6.1.3).

\subsection{The covariant derivative, it's adjoint and the Laplacian}

Let $M$ be an oriented Riemannian manifold. Recall that for a vector field $v \in \Gamma(M, T M)$ and sections $s, s^{\prime} \in \Gamma(M, E)$ of a Riemannian vector bundle $E \rightarrow M$ with metric compatible connection $\nabla$, we have the following standard computation:

$$
\begin{aligned}
\left\langle\nabla s, v \otimes s^{\prime}\right\rangle & =\left\langle\nabla_{v} s, s^{\prime}\right\rangle=-\left\langle s, \nabla_{v} s^{\prime}\right\rangle+d\left(\left\langle s, s^{\prime}\right\rangle\right)(v) \\
& =-\left\langle s, \nabla_{v} s^{\prime}\right\rangle+\operatorname{div}^{\nabla^{M}}\left(\left\langle s, s^{\prime}\right\rangle v\right)-\left\langle s, s^{\prime}\right\rangle \operatorname{div}^{\nabla^{M}}(v) \\
& =-\left\langle s, \nabla_{v} s^{\prime}\right\rangle-\left\langle s, \operatorname{div}^{\nabla^{M}}(v) s^{\prime}\right\rangle+\left\langle s, \mathcal{T}^{M}(v) s^{\prime}\right\rangle+\operatorname{div}^{\nabla^{L C}}\left(\left\langle s, s^{\prime}\right\rangle v\right) \\
& =-\left\langle s, \operatorname{tr}\left(\nabla\left(v \otimes s^{\prime}\right)\right)\right\rangle+\left\langle s, \mathcal{T}^{M}(v) s^{\prime}\right\rangle+\operatorname{div}^{\nabla^{L C}}\left(\left\langle s, s^{\prime}\right\rangle v\right) .
\end{aligned}
$$

If $s, s^{\prime}$ are compactly supported, integration yields

$$
\left\langle\nabla s, v \otimes s^{\prime}\right\rangle_{L^{2}}=-\left\langle s, \operatorname{tr}\left(\nabla\left(v \otimes s^{\prime}\right)\right)\right\rangle_{L^{2}}+\left\langle s, \mathcal{T}^{\nabla^{M}}(v) s^{\prime}\right\rangle_{L^{2}}+\int_{\partial M}\left\langle s,\langle\overrightarrow{\mathfrak{n}}, v\rangle s^{\prime}\right\rangle .
$$

Therefore, one usually denotes

$$
\nabla^{*} \alpha:=-\operatorname{tr}\left(\nabla^{T^{*} M \otimes E} \alpha\right)+\left\langle\mathcal{T}^{\nabla^{M}}, \alpha\right\rangle
$$


for any $\alpha \in \Omega^{1}(M, E)$, where $\operatorname{tr}: T^{*} M \otimes T^{*} M \rightarrow \mathbb{R}$ is induced by the metric. If $M$ is closed, then $\nabla^{*}: \Omega^{1}(M, E) \rightarrow \Gamma(M, E)$ is the formal $L^{2}$-adjoint of $\nabla$.

The previous discussion and Remark 4.3.3 justify the following generalization: Let $M$ be a Riemannian manifold and $E \rightarrow M$ a $H$-equivariant vector bundle with $H$-equivariant connector $\mathcal{K}^{E}$. Let $Q \rightarrow Z$ be principal $H$-bundle which is a reduction of the bundle of oriented orthonormal frames $P_{S O(m)} \rightarrow Z$. Furthermore, let $\varphi$ be a connection on $P_{S O(m)}$ with torsion $T^{\varphi}$ and torsion 1 -form $\mathcal{T}^{\varphi}(v):=-\operatorname{tr}\left(\iota_{v} T^{\varphi}\right)$, interpreted as an equivariant $\operatorname{map} \mathcal{T}^{\varphi} \in C^{\infty}\left(Q,\left(\mathbb{R}^{m}\right)^{\vee}\right)^{H}$. Denote $\mathscr{N}=C^{\infty}(Q, M)^{H}$.

6.1.1 Definition. Let $u \in \mathscr{N}, v \in C^{\infty}\left(Q,\left(\mathbb{R}^{m}\right)^{\vee} \otimes E\right)^{H}, A \in \mathscr{A}$ lifing a connection $\varphi$. We define

$$
\begin{aligned}
d_{A, \mathcal{K}}^{E, *}: C^{\infty}\left(Q,\left(\mathbb{R}^{m}\right)^{\vee} \otimes E\right)^{H} & \rightarrow C^{\infty}(Q, E)^{H}, \\
\alpha & \mapsto d_{A, \mathcal{K}}^{E, *} \alpha:=\left\langle\alpha, \mathcal{T}^{\varphi}\right\rangle-\operatorname{tr}\left(d_{A, \mathcal{K}}^{\left(\mathbb{R}^{m}\right)^{\vee} \otimes E} \alpha\right),
\end{aligned}
$$

and, in the case $E=T M$, and $\mathcal{K}$ the connector of the Levi-Civita connection, the Laplacian is

$$
\begin{aligned}
\Delta_{A, \mathcal{K}}^{M}: C^{\infty}(Q, M)^{H} & \rightarrow C^{\infty}(Q, T M)^{H} \\
u & \mapsto d_{A, \mathcal{K}}^{T M, *} d_{A}^{M} u=\left\langle d_{A} u, \mathcal{T}^{\varphi}\right\rangle-\operatorname{tr}\left(d_{A, \mathcal{K}}^{\left(\mathbb{R}^{m}\right)^{\vee} \otimes T M}\left(d_{A}^{M} u\right)\right),
\end{aligned}
$$

where tr: $\left(\mathbb{R}^{m}\right)^{\vee} \otimes\left(\mathbb{R}^{m}\right)^{\vee} \cong\left(\mathbb{R}^{m}\right)^{\vee} \otimes \mathbb{R}^{m} \rightarrow \mathbb{R}$ is induced by the standard metric on $\mathbb{R}^{m}$.

6.1.2 Remark. Note that

$$
d_{A, \mathcal{K}}^{E, *}: C^{\infty}\left(Q,\left(\mathbb{R}^{m}\right)^{\vee} \otimes E\right)_{u}^{H} \rightarrow C^{\infty}(Q, E)_{u}^{H},
$$

and, if $E=T M$

$$
\Delta_{A, \mathcal{K}}^{M}: C^{\infty}(Q, M)^{H} \rightarrow C^{\infty}(Q, T M)^{H}
$$

is a section of the infinite-dimensional vector bundle $\pi_{M}: C^{\infty}(Q, T M)^{H} \rightarrow C^{\infty}(Q, M)^{H}$, i.e. $\Delta_{A, \mathcal{K}}^{M} u \in C^{\infty}(Q, T M)_{u}^{H}$.

The following statement shows that this generalization is reasonably behaved, in particular, how the linearization of the Laplacian is related to the Laplacian of the linearized covariant derivative.

6.1.3 Proposition. $\Delta_{A, \mathcal{K}}^{M, l i n}:=\nabla^{\mathscr{N}}\left(\Delta_{A, \mathcal{K}}^{M}\right)=\mathcal{K} \circ T\left(\Delta_{A, \mathcal{K}}^{M}\right): C^{\infty}(Q, T M)^{H} \rightarrow C^{\infty}(Q, T M)^{H}$ is given by

$$
\Delta_{A, \mathcal{K}}^{M, l i n} v=d_{A, \mathcal{K}}^{T M, *} d_{A, \mathcal{K}}^{T M} v-\operatorname{tr}^{h o r}\left(u^{*} \iota_{v} F^{\mathcal{K}}\right),
$$

where $u=\pi_{M} \circ v$ and $\operatorname{tr}^{\text {hor }}\left(u^{*} \iota_{v} F^{\mathcal{K}}\right):=\sum_{\ell} F^{\mathcal{K}}\left(v, T u\left(\tilde{e}_{\ell}\right)\right) T u\left(\tilde{e}_{\ell}\right)$ for an orthonormal basis $\left\{\tilde{e}_{\ell}\right\}$ of horizontal vector fields on $Q$.

Proof. Since $d_{A, \mathcal{K}}^{T M, *} v=\left\langle v, \mathcal{T}^{\varphi}\right\rangle-\operatorname{tr}\left(d_{A, \mathcal{K}}^{\mathbb{R}^{m} \otimes T M} v\right)$ and $T d_{A, \mathcal{K}}^{\left(\mathbb{R}^{m}\right)^{\vee} \otimes T M}=\left(\operatorname{id}_{\left(R^{m}\right)^{\vee} \otimes\left(\mathbb{R}^{m}\right)^{\vee} \otimes((T \mathcal{K}) \circ}\right.$ $\left.\left.\kappa_{T M}\right)\right) \circ d_{A}^{T T M}$, we obtain

$$
\begin{aligned}
& T\left(d_{A, \mathcal{K}}^{T M, *}\right) f_{\alpha}=\left\langle f_{\alpha}, \mathcal{T}^{\varphi}\right\rangle-\operatorname{tr}\left(T d_{A, \mathcal{K}}^{\mathbb{R}^{m} \otimes T M} f_{\alpha}\right)
\end{aligned}
$$

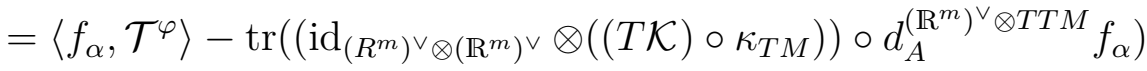

$$
\begin{aligned}
& =\left\langle f_{\alpha}, \mathcal{T}^{\varphi}\right\rangle-(T \mathcal{K}) \circ \kappa_{T M} \circ \operatorname{tr}\left(d_{A}^{\left(\mathbb{R}^{m}\right)^{\vee} \otimes T T M} f_{\alpha}\right) \text {. }
\end{aligned}
$$


for all $f_{\alpha} \in C^{\infty}\left(Q,\left(\mathbb{R}^{m}\right)^{\vee} \otimes T T M\right)^{H}$, and therefore

$$
\mathcal{K} \circ\left(T d_{A, \mathcal{K}}^{T M, *}\right) f_{\alpha}=\mathcal{K}\left\langle f_{\alpha}, \mathcal{T}^{\varphi}\right\rangle-\mathcal{K} \circ(T \mathcal{K}) \circ \kappa_{T M} \circ \operatorname{tr}\left(d_{A}^{\left(\mathbb{R}^{m}\right)^{\vee} \otimes T T M} f_{\alpha}\right) .
$$

Using this and $T\left(d_{A}^{M}\right)=\left(\operatorname{id}_{\left(\mathbb{R}^{m}\right)^{\vee}} \otimes \kappa_{M}\right) \circ d_{A}^{T M}$, we have

$$
\begin{aligned}
& \Delta_{A, \mathcal{K}}^{M, \operatorname{lin}}(v)=\mathcal{K} \circ T \Delta_{A, \mathcal{K}} v=\mathcal{K} \circ T\left(d_{A, \mathcal{K}}^{T M, *} d_{A}^{M}\right)(v)=\mathcal{K} \circ T\left(d_{A, \mathcal{K}}^{T M, *}\right) \circ T\left(d_{A}^{M}\right)(v) \\
& =\left\langle\left(\operatorname{id}_{\left(\mathbb{R}^{m}\right) \vee} \otimes\left(\mathcal{K} \circ \kappa_{M}\right)\right) \circ d_{A}^{T M} v, \mathcal{T}^{\varphi}\right\rangle \\
& -\mathcal{K} \circ(T \mathcal{K}) \circ \kappa_{T M} \circ \operatorname{tr}\left(d_{A}^{\left(\mathbb{R}^{m}\right)^{\vee} \otimes T T M}\left(\left(\operatorname{id}_{\left(\mathbb{R}^{m}\right)^{\vee}} \otimes \kappa_{M}\right) \circ d_{A}^{T M} v\right)\right) \\
& =\left\langle d_{A, \mathcal{K}}^{T M} v, \mathcal{T}^{\varphi}\right\rangle-\mathcal{K} \circ(T \mathcal{K}) \circ \kappa_{T M} \circ T\left(\kappa_{M}\right) \circ \operatorname{tr}\left(d_{A}^{\left(\mathbb{R}^{m}\right)^{\vee} \otimes T T M}\left(d_{A}^{T M} v\right)\right) \text {. }
\end{aligned}
$$

Furthermore, we know that

$$
\begin{aligned}
& d_{A, \mathcal{K}}^{\left(\mathbb{R}^{3}\right)^{\vee} \otimes T M, *} d_{A, \mathcal{K}}^{T M} v \\
& =\left\langle d_{A, \mathcal{K}}^{T M} v, \mathcal{T}^{\varphi}\right\rangle-\operatorname{tr}\left(d_{A, \mathcal{K}}^{\left(\mathbb{R}^{m}\right)^{\vee} \otimes T M} d_{A, \mathcal{K}}^{T M} v\right) \\
& =\left\langle d_{A, \mathcal{K}}^{T M} v, \mathcal{T}^{\varphi}\right\rangle-\operatorname{tr}\left(\left(\operatorname{id}_{\left(\mathbb{R}^{m}\right)^{\vee}} \otimes \mathcal{K}\right) \circ d_{A}^{\left(\mathbb{R}^{m}\right)^{\vee} \otimes T M}\left(\left(\operatorname{id}_{\left(\mathbb{R}^{m}\right)^{\vee}} \otimes \mathcal{K}\right) \circ d_{A}^{T M} v\right)\right) \\
& =\left\langle d_{A, \mathcal{K}}^{T M} v, \mathcal{T}^{\varphi}\right\rangle-\operatorname{tr}\left(\left(\operatorname{id}_{\left(\mathbb{R}^{m}\right) \vee} \otimes(\mathcal{K} \circ T(\mathcal{K}))\right) \circ d_{A}^{\left(\mathbb{R}^{m}\right)^{\vee} \otimes T T M}\left(d_{A}^{T M} v\right)\right) \\
& =\left\langle d_{A, \mathcal{K}}^{T M} v, \mathcal{T}^{\varphi}\right\rangle-\mathcal{K} \circ(T \mathcal{K}) \circ \operatorname{tr}\left(d_{A}^{\left(\mathbb{R}^{m}\right)^{\vee} \otimes T T M}\left(d_{A}^{T M} v\right)\right)
\end{aligned}
$$

for all $v \in C^{\infty}(Q, T M)^{H}$.

Note that given three tangent vectors $v_{1}, v_{2}, v_{3} \in T_{x} M$, we can extend them to (locally) commuting vector field $V_{1}, V_{2}, V_{3} \in \Gamma(M, T M)$ with $\left.V_{1}\right|_{x}=v_{1},\left.V_{2}\right|_{x}=v_{2},\left.V_{3}\right|_{x}=v_{3}$. Then consider $c(s, t, u):=\Phi_{s}^{V_{3}} \Phi_{t}^{V_{2}} \Phi_{u}^{V_{1}}(x)$, where $\Phi_{s}^{V_{3}}, \Phi_{t}^{V_{2}}, \Phi_{u}^{V_{1}}$ are the flows of $V_{1}, V_{2}, V_{3}$, respectively. Then

$$
\begin{aligned}
T \kappa_{M} T^{2} V_{3}\left(T V_{2}\left(\left.V_{1}\right|_{x}\right)\right) & =T \kappa_{M} \frac{d}{d u} T V_{3}\left(\left.\left.V_{2}\right|_{\left.\Phi_{t}^{V_{1}}(x)\right)}\right|_{u=0}=\left.\left.\left.T \kappa_{M} \frac{d}{d u} \frac{d}{d t} V_{3}\right|_{\Phi_{u}^{V_{2}}\left(\Phi_{t}^{V_{1}}(x)\right)}\right|_{t=0}\right|_{u=0}\right. \\
& =\left.\left.\left.T \kappa_{M} \frac{d}{d u} \frac{d}{d t} \frac{d}{d s} c(s, t, u)\right|_{s=0}\right|_{t=0}\right|_{u=0}=\left.\left.\left.\frac{d}{d u} \kappa_{M} \frac{d}{d t} \frac{d}{d s} c(s, t, u)\right|_{s=0}\right|_{t=0}\right|_{u=0} \\
& =\left.\left.\left.\frac{d}{d u} \frac{d}{d s} \frac{d}{d t} c(s, t, u)\right|_{t=0}\right|_{s=0}\right|_{u=0}=\left.\left.\frac{d}{d u} \frac{d}{d s} d \Phi_{s}^{V_{3}}\left(\left.V_{2}\right|_{\Phi_{s}^{V_{1}}(x)}\right)\right|_{s=0}\right|_{u=0} \\
& =\left.\left.\frac{d}{d u} \frac{d}{d s}\left(\left.V_{2}\right|_{\Phi_{s}^{V_{3}}\left(\Phi_{s}^{V_{1}}(x)\right)}\right)\right|_{s=0}\right|_{u=0}=\left.\frac{d}{d u} T V_{2}\left(\left.V_{3}\right|_{\Phi_{s}^{V_{1}}(x)}\right)\right|_{u=0} \\
& =T^{2} V_{2}\left(T V_{3}\left(\left.V_{1}\right|_{x}\right)\right) .
\end{aligned}
$$

Using the formula for the curvature from [KMS93, Thm. 37.15], we have for vector fields $V_{1}, V_{2}, V_{3} \in \Gamma(M, T M)$ :

$$
\begin{aligned}
\left.F^{\mathcal{K}}\left(\left.V_{3}\right|_{x},\left.V_{1}\right|_{x}\right) V_{2}\right|_{x} & =\left(\mathcal{K} \circ(T \mathcal{K}) \circ \kappa_{T M}-\mathcal{K} \circ(T \mathcal{K})\right) T^{2} V_{2} \circ T V_{3}\left(\left.V_{1}\right|_{x}\right) \\
& =\left(\mathcal{K} \circ(T \mathcal{K}) \circ \kappa_{T M}-\mathcal{K} \circ(T \mathcal{K})\right) T \kappa_{M} T^{2} V_{3}\left(T V_{2}\left(\left.V_{1}\right|_{x}\right)\right) \\
& =\left(\mathcal{K} \circ(T \mathcal{K}) \circ \kappa_{T M} \circ T \kappa_{M}-\mathcal{K} \circ(T \mathcal{K})\right) T^{2} V_{3}\left(T V_{2}\left(\left.V_{1}\right|_{x}\right)\right) .
\end{aligned}
$$

The same argument as in the second part of [KMS93, Thm. 37.15], namely computing in a local trivialization of the bundle, shows that this identity can be extended to sections of the pullback bundle:

$$
\left(\mathcal{K} \circ(T \mathcal{K}) \circ \kappa_{T M}-\mathcal{K} \circ(T \mathcal{K})\right) \circ T\left(\kappa_{M}\right) \circ T^{2} v\left(T \tilde{e}_{\ell}\left(\left.\tilde{e}_{\ell}\right|_{p}\right)\right)=F^{\mathcal{K}}\left(v, T u\left(\tilde{e}_{\ell}\right)\right) T u\left(\tilde{e}_{\ell}\right) .
$$


Therefore

$$
\begin{aligned}
& \left(\mathcal{K} \circ(T \mathcal{K}) \circ \kappa_{T M} \circ T\left(\kappa_{M}\right)-\mathcal{K} \circ(T \mathcal{K})\right) \circ \operatorname{tr}\left(d_{A}^{\left(\mathbb{R}^{m}\right)^{\vee} \otimes T T M}\left(d_{A}^{T M} v\right)\right)(p) \\
= & \left(\mathcal{K} \circ(T \mathcal{K}) \circ \kappa_{T M}-\mathcal{K} \circ(T \mathcal{K})\right) \circ T\left(\kappa_{M}\right) \circ \operatorname{tr}\left(d_{A}^{\left(\mathbb{R}^{m}\right)^{\vee} \otimes T T M}\left(d_{A}^{T M} v\right)\right)(p) \\
= & \left(\mathcal{K} \circ(T \mathcal{K}) \circ \kappa_{T M}-\mathcal{K} \circ(T \mathcal{K})\right) \circ T\left(\kappa_{M}\right) \circ T^{2} v\left(T \tilde{e}_{\ell}\left(\left.\tilde{e}_{\ell}\right|_{p}\right)\right) \\
= & F^{\mathcal{K}}\left(v, T u\left(\tilde{e}_{\ell}\right)\right) T u\left(\tilde{e}_{\ell}\right)(p) \\
= & \operatorname{tr}^{h o r}\left(u^{*} \iota_{v} F^{\mathcal{K}}\right)(p) .
\end{aligned}
$$

Finally, combining these formulae, we obtain

$$
\begin{aligned}
\Delta_{A, \mathcal{K}}^{M, l i n}(v)= & \left\langle d_{A, \mathcal{K}}^{T M} v, \mathcal{T}^{\varphi}\right\rangle-\mathcal{K} \circ(T \mathcal{K}) \circ \kappa_{T M} \circ T\left(\kappa_{M}\right) \circ \operatorname{tr}\left(d_{A}^{\left(\mathbb{R}^{m}\right)^{\vee} \otimes T T M}\left(d_{A}^{T M} v\right)\right) \\
= & d_{A, \mathcal{K}}^{\left(\mathbb{R}^{3}\right)^{\vee} \otimes T M, *} d_{A, \mathcal{K}}^{T M} v \\
& -\left(\mathcal{K} \circ(T \mathcal{K}) \circ \kappa_{T M} \circ T\left(\kappa_{M}\right)-\mathcal{K} \circ(T \mathcal{K})\right) \circ \operatorname{tr}\left(d_{A}^{\left(\mathbb{R}^{m}\right)^{\vee} \otimes T T M}\left(d_{A}^{T M} v\right)\right) \\
= & d_{A, \mathcal{K}}^{\left(\mathbb{R}^{3}\right)^{\vee} \otimes T M, *} d_{A, \mathcal{K}}^{T M} v-\operatorname{tr}^{h o r}\left(u^{*} \iota_{v} F^{\mathcal{K}}\right) .
\end{aligned}
$$

6.1.4 Remark. If $Z$ is an interval, $Q \rightarrow Z$ is the trivial bundle and $H$ acts trivially on $M$, then $\Delta_{A, \mathcal{K}}^{M} u=0$ if and only if $u$ is a geodesic. Furthermore, $\Delta_{A, \mathcal{K}}^{M, l i n} v=0$ if and only if $v: Z \rightarrow T M$ is a Jacobi vector field along $u: Z \rightarrow M$. If $Z=S^{1}=\mathbb{R} / \mathbb{Z}$, then the same holds and additionally, $u$ is a periodic geodesic.

More generally, if $H$ acts trivially on $M$ and $A$ lifts the Levi-Civita connection, then $\Delta_{A, \mathcal{K}}^{M} u=0$ if and only if $u: Z \rightarrow M$ is harmonic. Harmonic maps have been studied intensively in the literature, see for instance [EL95], [Xin96] for introductions to the subject. The following Corollary is of cause well-known in this situation.

Therefore, the general case above is a equivariant generalization of harmonic maps, and a solution of $\Delta_{A, \mathcal{K}}^{M} u=0$ can equivalently be understood as harmonic sections in a (nonlinear) fibre bundle.

We are of cause mostly iterested in the case $m \in\{3,4\}, H=\operatorname{Spin}_{\varepsilon}^{G}(m), M$ a hyperkähler manifold with permuting $\operatorname{Spin}_{\varepsilon}^{G}(m)$-action. In this case, the Lichnerowicz-Weitzenböck formula (Theorem 6.2.1, Theorem 6.7.1) compares the Laplacian $\Delta_{A, \mathcal{K}}^{M}$ to the Dirac Laplacian.

6.1.5 Corollary. Assume $Z$ closed and consider the energy $E(u):=\frac{1}{2} \int_{Z}\left\|d_{A}^{M} u\right\|^{2}$. Then

$$
d E(v)=\int_{Z}\left\langle\Delta_{A, \mathcal{K}}^{M} u, v\right\rangle
$$

and hence $\operatorname{grad}(E)(u)=\Delta_{A, \mathcal{K}}^{M} u$, and the Hessian of $E$ is

$$
\operatorname{Hess}(E)(v, w)=\int_{Z}\left\langle d_{A, \mathcal{K}}^{T M, *} d_{A, \mathcal{K}}^{T M} v, w\right\rangle-\int_{Z}\left\langle\operatorname{tr}^{h o r}\left(u^{*} \iota_{v} F^{\mathcal{K}}\right), w\right\rangle
$$

This generalizes well-known results in the non-equivariant case (cf. [Xin96, Section 1.4.3], [EL95, Section 3.8]). 
Proof. Using the $L^{2}$-metric on $C^{\infty}(Q, M)^{H}$ and its Levi-Civita connection $\nabla$ (as in Remark 4.1.6), we can compute

$$
\begin{aligned}
d E(v) & \left.=\nabla_{v}(E)=\frac{1}{2} \nabla_{v}\left(g\left(d_{A}^{M} u, d_{A}^{M} u\right)\right)=g\left(\nabla_{v}\left(d_{A}^{M}\right), d_{A}^{M} u\right)\right) \\
& =g\left(d_{A, \mathcal{K}}^{T M} v, d_{A}^{M} u\right)=g\left(v, d_{A, \mathcal{K}}^{T M} d_{A}^{M} u\right)=g\left(v, \Delta_{A, \mathcal{K}}^{M} u\right)
\end{aligned}
$$

and

$$
\nabla_{v}(\operatorname{grad}(E))=\nabla_{v}\left(\Delta_{A, \mathcal{K}}^{M}\right)=d_{A, \mathcal{K}}^{T M, *} d_{A, \mathcal{K}}^{T M} v+\sum_{\ell} F^{\mathcal{K}}\left(v, T u\left(\tilde{e}_{\ell}\right)\right) T u\left(\tilde{e}_{\ell}\right)
$$

\subsection{Lichnerowicz-Weitzenböck formulae and curvature identities}

Consider a hyperkähler manifold $M$ with a permuting action of $\operatorname{Spin}_{\varepsilon}^{G}(3)$ and let $\mathcal{K}: T T M \rightarrow T M$ be the connector of the Levi-Civita connection.

Fix a $\operatorname{Spin}_{\varepsilon}^{G}(3)$-structure $Q_{3} \rightarrow Y$ over a oriented Riemannian 3-manifold $Y$. Let $A \in \mathscr{A}_{3}$ be a connection 1-form on $Q$ which lifts a metric connection $\varphi \in \mathscr{A}\left(P_{S O(3)}\right)$, i.e. $\operatorname{pr}_{\mathfrak{s p}(1)} A=\nu^{-1} \pi_{S O(3)}^{*} \varphi$, where $\nu: \mathfrak{s p}(1) \rightarrow \mathfrak{s o}(3)$ is the isomorphism of Lie algebras induced by the 2 -fold covering $S p(1) \cong \operatorname{Spin}(3) \rightarrow S O(3)$. We denote the $\mathfrak{g}$-component of $A$ by $a$. Finally, let $\theta_{Y}$ denote the canonical 1-form $\theta_{Y} \in \Omega^{1}\left(Q, \mathbb{R}^{3}\right)^{\operatorname{Spin}_{\varepsilon}^{G}(3)}$.

Using this notation, we have the following:

\subsubsection{Theorem (Lichnerowicz-Weitzenböck formulae).}

Let $u \in C^{\infty}\left(Q_{3}, M\right)^{\operatorname{Spin}_{\varepsilon}^{G}(3)}$ a spinor, $v \in C^{\infty}\left(Q_{3}, T M\right)^{\operatorname{Spin}_{\varepsilon}^{G}(3)}$ satisfying $\pi_{M} \circ v=u$ and $A \in \mathscr{A}$. Then

1. Lichnerowicz-Weitzenböck formula for generalized Dirac operator:

$$
\begin{aligned}
\mathscr{D}_{A}^{l i n, u, *} \mathscr{D}_{A} u= & \Delta_{A, \mathcal{K}}^{M} u+\left.\frac{s_{Y}}{4} \chi_{0}\right|_{u}+\frac{1}{2}\left\langle\left.\chi_{2}\right|_{u}, \operatorname{Ric}_{0}\right\rangle+\left\langle\left.\mathcal{Y}\right|_{u}, * F_{a}\right\rangle \\
& -\left\langle d_{A}^{M} u, \mathcal{T}^{\varphi}\right\rangle+c_{3}\left(\left\langle * T^{\varphi}, d_{A}^{M} u\right\rangle\right)-c_{3}\left(\mathcal{T}^{\varphi} \otimes \mathscr{D}_{A} u\right) .
\end{aligned}
$$

2. Lichnerowicz-Weitzenböck formula for linearized Dirac operator:

$$
\begin{aligned}
\mathscr{D}_{A}^{\text {lin,u,*}} \mathscr{D}_{A}^{\text {lin,u}} v= & d_{A, \mathcal{K}}^{T M, *} d_{A, \mathcal{K}}^{T M} v+\left.\frac{s_{Y}}{4} \nabla_{v}^{\mathcal{K}}\left(\chi_{0}\right)\right|_{u}+\frac{1}{2}\left\langle\left.\nabla_{v}^{\mathcal{K}}\left(\chi_{2}\right)\right|_{u}, \operatorname{Ric}_{0}\right\rangle \\
& \left.+\left\langle\left.\nabla_{v}^{\mathcal{K}}(\mathcal{Y})\right|_{u}, * F_{a}\right\rangle\right)-c_{3}\left(* \iota_{\text {hor }}^{2} u^{*} F_{\mathcal{K}} v\right) \\
& -\left\langle d_{A, \mathcal{K}}^{T M} v, \mathcal{T}^{\varphi}\right\rangle+c_{3}\left(\left\langle d_{A, \mathcal{K}}^{T M} v, * T^{\varphi}\right\rangle\right)-c_{3}\left(\mathcal{T}^{\varphi} \otimes \mathscr{D}_{A}^{\text {lin,u}} v\right) .
\end{aligned}
$$

3. Norms and $L^{2}$-Lichnerowicz-Weitzenböck formula:

$$
\begin{aligned}
\left\|\mathscr{D}_{A} u\right\|^{2}= & \left\|d_{A}^{M} u\right\|^{2}-2\left\langle\Phi_{3}(u), * F_{a}\right\rangle+\frac{s_{Y}}{2} \rho_{0} \circ u+\left\langle\rho_{2} \circ u, \text { Ric }_{0}\right\rangle \\
& +2 * d\left\langle\theta_{Y} \wedge\left(u^{*} \gamma\right)_{h o r}\right\rangle-2 *\left\langle T^{\varphi} \wedge\left(u^{*} \gamma\right)_{h o r}\right\rangle,
\end{aligned}
$$


and, if $u \in C^{\infty}(Q, M)^{\operatorname{Spin}_{\varepsilon}^{G}(3)}$ has compact support

$$
\begin{aligned}
\left\|\mathscr{D}_{A} u\right\|_{L^{2}}^{2}= & \left\|d_{A}^{M} u\right\|_{L^{2}}^{2}-2\left\langle\Phi_{3}(u), * F_{a}\right\rangle_{L^{2}}+\int_{Y} \frac{s_{Y}}{2} \rho_{0} \circ u+\int_{Y}\left\langle\rho_{2} \circ u, \operatorname{Ric}_{0}\right\rangle \\
& +2 \int_{\partial Y}\left\langle\theta_{Y} \wedge\left(u^{*} \gamma\right)_{h o r}\right\rangle-2 \int_{Y}\left\langle T^{\varphi} \wedge\left(u^{*} \gamma\right)_{h o r}\right\rangle .
\end{aligned}
$$

Here $\iota_{\text {hor }}^{2}: \Omega^{2}(Q \text {, End }(T M))_{h o r}^{\operatorname{Spin}_{\varepsilon}^{G}(3)} \stackrel{\sim}{\rightarrow} C^{\infty}\left(Q, \wedge^{2}\left(\mathbb{R}^{3}\right)^{\vee} \otimes \text { End }(T M)\right)^{\operatorname{Spin}_{\varepsilon}^{G}(3)}$ and $*$ denotes the 3-dimensional Hodge star operator $*: \wedge^{2}\left(\mathbb{R}^{3}\right)^{\vee} \rightarrow\left(\mathbb{R}^{3}\right)^{\vee}$.

The proof, which will be given below, is similar to the one in the 4-dimensional case (cf. [Sch10, Thm. 4.7.1, Thm. 4.7.2] and [Pid04, Thm. 5.4]). A Lichnerowicz-Weitzenböck formula for a generalized Dirac operator in dimension 3 first appeared in [Tau99]. Note that we allow the metric connection $\varphi$ on $P_{S O(3)}$ to have torsion.

We start by reminding the reader of the curvature formulae ([Sch10, Lem. 2.4.1 and Lem. $\left.2.4 .2]^{1}\right)$.

6.2.2 Lemma (curvature formulae). Let $P \rightarrow Y$ a principal $H$-bundle, $V_{1}, V_{2} \in$ $\Gamma(P, T P), v \in C^{\infty}(P, T M)^{H}$ with $u:=\pi_{M} \circ v \in C^{\infty}(P, M)^{H}$

$$
\begin{gathered}
{\left[\nabla_{V_{1}}^{A, \mathcal{K}}, \nabla_{V_{2}}^{A, \mathcal{K}}\right] v-\nabla_{\left[V_{1}, V_{2}\right]}^{A, \mathcal{K}} v=F_{\mathcal{K}}\left(d_{A} u \circ V_{1}, d_{A} u \circ V_{2}\right) v-\mathcal{K}\left(\left.v_{F_{A}\left(V_{1}, V_{2}\right)}^{H}\right|_{v}\right),} \\
\nabla_{V_{1}}^{A, \mathcal{K}} \nabla_{V_{2}}^{A} u-\nabla_{V_{2}}^{A, \mathcal{K}} \nabla_{V_{1}}^{A} u-\nabla_{\left[V_{1}, V_{2}\right]}^{A} u=\Theta^{\mathcal{K}}\left(\nabla_{V_{1}}^{A} u, \nabla_{V_{2}}^{A} u\right)-\left.v_{F_{A}\left(V_{1}, V_{2}\right)}^{H}\right|_{u} .
\end{gathered}
$$

Here, $\nabla_{V}^{A} u:=T u\left(\operatorname{pr}_{\mathscr{H}_{A}}(V)\right)$ and $\nabla_{V}^{A, \mathcal{K}} v:=\mathcal{K}\left(T v\left(\operatorname{pr}_{\mathscr{H}_{A}}(V)\right)\right)$.

We will now return to our principal $\operatorname{Spin}_{\varepsilon}^{G}(3)$-bundle $Q \rightarrow Y$ with connection $A \in \mathscr{A}_{3}$, and let $M$ a hyperkähler manifold with permuting $\operatorname{Spin}_{\varepsilon}^{G}(3)$-action and connector $\mathcal{K}$ corresponding to the Levi-Civita connection. In particular, the torsion $\Theta^{\mathcal{K}}$ vanishes.

Note that $\left\langle d_{A}^{M} u(p), V\right\rangle=T u\left(\widetilde{\pi_{S O}(p)(V)}\right)=\nabla_{\tilde{V}}^{A} u(p)$, where $V \in \mathbb{R}^{m}, \pi_{S O}: Q \rightarrow P_{S O(m)}$ is the projection and $\tilde{V}_{p}:=\widetilde{\pi_{S O}(p)(v)} \in T_{p} Q$ is the horizontal lift of $\pi_{S O}(p)(v) \in T_{\pi(p)} Y$. Similarly, $\nabla_{\tilde{V}}^{A, \mathcal{K}} v=\left\langle d_{A, \mathcal{K}}^{T M} v, V\right\rangle$.

Let now $R^{\varphi}$ be the curvature of the metric connection $\varphi$ on $T Y \rightarrow Y$. Slightly abusing notation, we will use $R^{\varphi}$ for the 2-form in $\Omega^{2}(Q, \mathfrak{s o}(3))^{\operatorname{Spin}_{\varepsilon}^{G}(3)}$ as well as for the corresponding equivariant map in $C^{\infty}\left(Q, \wedge^{2}\left(\mathbb{R}^{3}\right)^{\vee} \otimes \mathfrak{s o}(3)\right)^{\operatorname{Spin}_{\varepsilon}^{G}(3)}$, implicitly using the isomorphism $\iota_{\text {hor }}^{2}: \Omega^{2}(Q, \mathfrak{s o}(3))_{h o r}^{\operatorname{Spin}_{\varepsilon}^{G}(3)} \rightarrow C^{\infty}\left(Q, \wedge^{2}\left(\mathbb{R}^{3}\right)^{\vee} \otimes \mathfrak{s o}(3)\right)^{\operatorname{Spin}_{\varepsilon}^{G}(3)}$. We proceed similarly with $F_{A}$ and $F_{a}$.

The following lemma shows how $\nu^{-1} * R^{\varphi} \in C^{\infty}\left(Q,\left(\mathbb{R}^{3}\right)^{\vee} \otimes \mathfrak{s p}(1)\right)^{\operatorname{Spin}_{\varepsilon}^{G}(3)}$ can be decomposed into scalar curvature and traceless Ricci curvature.

\subsubsection{Lemma.}

$$
\operatorname{pr}_{\mathbb{R}} \nu^{-1} * R^{\varphi}=-\frac{s_{Y}}{4} \frac{1}{3} \sum_{\ell=1}^{3} \zeta_{\ell} \otimes \zeta_{\ell} \quad \text { and } \quad \operatorname{pr}_{S_{0}^{2} \mathfrak{s p}(1)} \nu^{-1} * R^{\varphi}=\frac{1}{2} \text { Ric }_{0}
$$

\footnotetext{
${ }^{1}$ Note that [Sch10] uses a different sign convention for the fundamental vector fields.
} 
In particular,

$$
\left\langle\chi, \nu^{-1} * R^{\varphi}\right\rangle=\frac{s_{Y}}{4} \chi_{0}+\frac{1}{2}\left\langle\chi_{2}, \text { Ric }_{0}\right\rangle
$$

where we use the isomorphism $\mathfrak{s p}(1) \cong \mathbb{R}^{3}, \zeta_{\ell} \mapsto e_{\ell}$.

Even though it is clear from representation theory which components appear, the coefficients are crucial and we therefore do the computation explicitly in terms of the components of the curvature tensor.

Proof. We first compute $\operatorname{pr}_{\mathbb{R}} \nu^{-1} * R^{\varphi}$ : Let $R^{\varphi}=\sum_{k<\ell} R_{i j k \ell}^{\varphi} e_{i} \wedge e_{j} \otimes E_{k, \ell}$, where $E_{k, \ell} \in \mathfrak{s o}(3)$ maps $e_{k} \mapsto e_{\ell}, e_{\ell} \mapsto-e_{k}$ and the third basis vector to zero. Then

$$
\begin{aligned}
\nu^{-1} * R^{\varphi}= & \frac{1}{2}\left(R_{1212}^{\varphi} e_{3} \otimes \zeta_{3}-R_{1213}^{\varphi} e_{3} \otimes \zeta_{2}+R_{1223}^{\varphi} e_{3} \otimes \zeta_{1}\right. \\
& -R_{1312}^{\varphi} e_{2} \otimes \zeta_{3}+R_{1313}^{\varphi} e_{2} \otimes \zeta_{2}-R_{1323}^{\varphi} e_{2} \otimes \zeta_{1} \\
& \left.+R_{2312}^{\varphi} e_{1} \otimes \zeta_{3}-R_{2313}^{\varphi} e_{1} \otimes \zeta_{2}+R_{2323}^{\varphi} e_{1} \otimes \zeta_{1}\right) .
\end{aligned}
$$

Applying $\operatorname{pr}_{\mathbb{R}}$ yields

$$
\operatorname{pr}_{\mathrm{R}} \nu^{-1} * R^{\varphi}=\frac{1}{6}\left(R_{1212}^{\varphi}+R_{1313}^{\varphi}+R_{2323}^{\varphi}\right)\left(\sum_{\ell=1}^{3} \zeta_{\ell} \otimes \zeta_{\ell}\right)=-\frac{s_{Y}}{4} \frac{1}{3} \sum_{\ell=1}^{3} \zeta_{\ell} \otimes \zeta_{\ell} .
$$

In particular,

$$
\left\langle\chi, \operatorname{pr}_{\mathbb{R}} \nu^{-1} * R^{\varphi}\right\rangle=-\frac{s_{Y}}{4}\left\langle\chi, \frac{1}{3} \sum_{\ell=1}^{3} \zeta_{\ell} \otimes \zeta_{\ell}\right\rangle=\frac{s_{Y}}{4} \chi_{0} .
$$

Note that $\nu^{-1} * R^{\varphi}$ is symmetric, and hence $\nu^{-1} * R^{\varphi}=\operatorname{pr}_{\mathbb{R}} \nu^{-1} * R^{\varphi}+\operatorname{pr}_{S_{0}^{2} \mathfrak{s p}(1)} \nu^{-1} * R^{\varphi}$. In particular, $\operatorname{pr}_{S_{0}^{2} \mathfrak{s p}(1)} \nu^{-1} * R^{\varphi}=\nu^{-1} * R^{\varphi}-\operatorname{pr}_{\mathbb{R}} \nu^{-1} * R^{\varphi}$. Therefore,

$$
\begin{aligned}
& \operatorname{pr}_{S_{0}^{2} \mathfrak{s p}(1)} \nu^{-1} * R^{\varphi} \\
= & \frac{1}{2}\left(\left(\frac{2}{3} R_{1212}^{\varphi}-\frac{1}{3} R_{1313}^{\varphi}-\frac{1}{3} R_{2323}^{\varphi}\right) \zeta_{3} \otimes \zeta_{3}+\left(\frac{2}{3} R_{1313}^{\varphi}-\frac{1}{3} R_{1212}^{\varphi}-\frac{1}{3} R_{2323}^{\varphi}\right) \zeta_{2} \otimes \zeta_{2}\right. \\
& +\left(\frac{2}{3} R_{2323}^{\varphi}-\frac{1}{3} R_{1212}^{\varphi}-\frac{1}{3} R_{1313}^{\varphi}\right) \zeta_{1} \otimes \zeta_{1}-R_{1213}^{\varphi} \zeta_{3} \otimes \zeta_{2}-R_{1312}^{\varphi} \zeta_{2} \otimes \zeta_{3}+R_{1223}^{\varphi} \zeta_{3} \otimes \zeta_{1} \\
& \left.+R_{2312}^{\varphi} \zeta_{1} \otimes \zeta_{3}-R_{2313}^{\varphi} \zeta_{1} \otimes \zeta_{2}-R_{1323}^{\varphi} \zeta_{2} \otimes \zeta_{1}\right) .
\end{aligned}
$$

On the other hand, the Ricci curvature is

$$
\begin{aligned}
& R i c=\sum_{i, j=1}^{3} \sum_{\ell=1}^{3} R_{i \ell \ell j}^{\varphi} \zeta_{i} \otimes \zeta_{j} \\
= & R_{1221}^{\varphi} \zeta_{1} \otimes \zeta_{1}+R_{1331}^{\varphi} \zeta_{1} \otimes \zeta_{1}+R_{2112}^{\varphi} \zeta_{2} \otimes \zeta_{2}+R_{2332}^{\varphi} \zeta_{2} \otimes \zeta_{2}+R_{3113}^{\varphi} \zeta_{3} \otimes \zeta_{3}+R_{3223}^{\varphi} \zeta_{3} \otimes \zeta_{3} \\
& +R_{1332}^{\varphi} \zeta_{1} \otimes \zeta_{2}+R_{2331}^{\varphi} \zeta_{2} \otimes \zeta_{1}+R_{1223}^{\varphi} \zeta_{1} \otimes \zeta_{3}+R_{3221}^{\varphi} \zeta_{3} \otimes \zeta_{1}+R_{2113}^{\varphi} \zeta_{2} \otimes \zeta_{3}+R_{3112}^{\varphi} \zeta_{3} \otimes \zeta_{2},
\end{aligned}
$$

and therefore, the traceless part of the Ricci cuvature is

$$
\begin{aligned}
\text { Ric }_{0}= & R i c-\frac{s_{Y}}{3} \sum_{\ell=1}^{3} \zeta_{\ell} \otimes \zeta_{\ell} \\
= & \left(\frac{1}{3} R_{1221}^{\varphi}+\frac{1}{3} R_{1331}^{\varphi}-\frac{2}{3} R_{2332}^{\varphi}\right) \zeta_{1} \otimes \zeta_{1}+\left(\frac{1}{3} R_{1221}^{\varphi}+\frac{1}{3} R_{2332}^{\varphi}-\frac{2}{3} R_{1331}^{\varphi}\right) \zeta_{2} \otimes \zeta_{2} \\
& +\left(\frac{1}{3} R_{1331}^{\varphi}+\frac{1}{3} R_{2332}^{\varphi}-\frac{2}{3} R_{1221}^{\varphi}\right) \zeta_{3} \otimes \zeta_{3}+R_{1332}^{\varphi} \zeta_{1} \otimes \zeta_{2}+R_{2331}^{\varphi} \zeta_{2} \otimes \zeta_{1}+R_{1223}^{\varphi} \zeta_{1} \otimes \zeta_{3} \\
& +R_{3221}^{\varphi} \zeta_{3} \otimes \zeta_{1}+R_{2113}^{\varphi} \zeta_{2} \otimes \zeta_{3}+R_{3112}^{\varphi} \zeta_{3} \otimes \zeta_{2} \\
= & 2 \operatorname{pr}_{S_{0}^{2} \mathfrak{s p}(1)} \nu^{-1} * R^{\varphi} .
\end{aligned}
$$


Combining all these, we finally obtain

$$
\begin{aligned}
\left\langle\chi, \nu^{-1} * R^{\varphi}\right\rangle & =\left\langle\chi, \operatorname{pr}_{\mathbb{R}} \nu^{-1} * R^{\varphi}\right\rangle+\left\langle\chi, \operatorname{pr}_{S_{0}^{2} \mathfrak{s p}(1)} \nu^{-1} * R^{\varphi}\right\rangle=\frac{s_{Y}}{4} \chi_{0}+\frac{1}{2}\left\langle\chi, \text { Ric }_{0}\right\rangle \\
& =\frac{s_{Y}}{4} \chi_{0}+\frac{1}{2}\left\langle\chi_{2}, \text { Ric }_{0}\right\rangle .
\end{aligned}
$$

\subsection{Lichnerowicz-Weitzenböck formula}

We will now use the curvature identities above to prove the Lichnerowicz-Weitzenböck formula

$$
\begin{aligned}
\mathscr{D}_{A}^{l i n, u, *} \mathscr{D}_{A} u= & \Delta_{A, \mathcal{K}}^{M} u+\left.\frac{s_{Y}}{4} \chi_{0}\right|_{u}+\frac{1}{2}\left\langle\left.\chi_{2}\right|_{u}, \operatorname{Ric}_{0}\right\rangle+\left\langle\left.\mathcal{Y}\right|_{u}, * F_{a}\right\rangle \\
& -\left\langle d_{A}^{M} u, \mathcal{T}^{\varphi}\right\rangle+c_{3}\left(\left\langle * T^{\varphi}, d_{A}^{M} u\right\rangle\right)-c_{3}\left(\mathcal{T}^{\varphi} \otimes \mathscr{D}_{A} u\right) .
\end{aligned}
$$

Proof (Theorem 6.2.1, part 1). First note that $\nu^{-1}$ maps the matrix $E_{i j} \in \mathfrak{s o}(3)$ to $\frac{1}{2} e_{i} e_{j} \in$ $\mathfrak{s p i n}(3) \subset C l_{3}$. Here $E_{i j} \in \mathfrak{s o}(3)$ sends $e_{i} \mapsto e_{j}, e_{j} \mapsto-e_{i}$ and the third basis vector the standard basis $\left(e_{1}, e_{2}, e_{3}\right)$ of $\mathbb{R}^{3}$ is send to zero. We obtain $F_{A}=\operatorname{pr}_{\mathfrak{s p i n}(3)} F_{A}+\operatorname{pr}_{\mathfrak{g}} F_{A}=$ $\nu^{-1} \pi_{S O}^{*} R^{\varphi}+F_{a}$. Moreover

$$
\nu^{-1} \pi_{S O}^{*} R^{\varphi}\left(\tilde{e}_{k}, \tilde{e}_{\ell}\right)=\nu^{-1} R_{k \ell}^{\varphi}=\sum_{i<j} \nu^{-1} R_{i j k \ell}^{\varphi} E_{i j}=\frac{1}{2} \sum_{i<j} R_{i j k \ell}^{\varphi} e_{i} e_{j},
$$

where $R^{\varphi}=\sum_{k<\ell} R_{k \ell}^{\varphi} e_{k} \wedge e_{l}$ with $R_{k \ell}^{\varphi} \in C^{\infty}\left(P_{S O}, \mathfrak{s o}(3)\right), R_{k \ell}^{\varphi}=\sum_{i<j} R_{i j k \ell}^{\varphi} E_{i j}$.

Fix a point $p \in Q_{3}, y:=\pi_{Y}(p)$ and let $X_{\ell}:=\pi_{S O}(p)\left(e_{\ell}\right) \in T_{y} Y$ for $\ell \in\{1,2,3\}$. Extend $X_{\ell} \in T_{y} Y$ to a local oriented orthonormal frame field given by the vector fields $X_{\ell} \in \Gamma(Y, T Y)$. Since $T Y$ is the associated bundle $T Y=Q_{3} \times_{S p i n}^{G}(3) \mathbb{R}^{3}$, these correspond to $\operatorname{Spin}_{\varepsilon}^{G}(3)$-equivariant maps $f_{\ell}: Q_{3} \rightarrow \mathbb{R}^{3}$. In particular, $X_{\ell}=\pi_{S O}(p)\left(e_{\ell}\right)$ implies that $f_{\ell}(p)=e_{\ell}$. More generally, for a vector field $X \in \Gamma(Y, T Y)$, denote the corresponding equivariant map by $f_{X} \in C^{\infty}\left(Q_{3}, \mathbb{R}^{3}\right)^{\operatorname{Spin}_{\varepsilon}^{G}(3)}$. With this notation at hand we can compute

$$
\begin{aligned}
\mathscr{D}_{A}^{l i n, u} \mathscr{D}_{A} u(p)= & \sum_{k=1}^{3} \sum_{\ell=1}^{3} c_{3}\left(e_{k}\right) \nabla_{\tilde{X}_{k}}^{A, \mathcal{K}}\left(c_{3}\left(\tilde{f}_{\ell}\right) \nabla_{\tilde{X}_{\ell}}^{A} u\right)(p) \\
= & \sum_{k=1}^{3} \sum_{\ell=1}^{3}\left(c_{3}\left(e_{k}\right) c_{3}\left(e_{\ell}\right) \nabla_{\tilde{X}_{k}}^{A, \mathcal{K}} \nabla_{\tilde{X}_{\ell}}^{A} u+c_{3}\left(e_{k}\right) c_{3}\left(f_{\nabla_{X_{k}}^{A} X_{\ell}}\right) \nabla_{\tilde{X}_{\ell}}^{A} u\right)(p) \\
= & \sum_{k=1}^{3} \sum_{\ell=1}^{3}\left(c_{3}\left(e_{k}\right) c_{3}\left(e_{\ell}\right) \nabla_{\tilde{X}_{k}}^{A, \mathcal{K}} \nabla_{\tilde{X}_{\ell}}^{A} u-c_{3}\left(e_{k}\right) c_{3}\left(e_{\ell}\right) \nabla_{\nabla_{X_{k}}^{A} X_{\ell}}^{A} u\right)(p) \\
= & -\operatorname{tr}\left(d_{A, \mathcal{K}}^{\left(\mathbb{R}^{3}\right)^{\vee} \otimes T M}\left(d_{A}^{M} u\right)\right)(p) \\
& +\sum_{1 \leq k \leq \ell \leq 3} c_{3}\left(e_{k}\right) c_{3}\left(e_{\ell}\right)\left(\nabla_{\tilde{X}_{k}}^{A, \mathcal{K}} \nabla_{\tilde{X}_{\ell}}^{A} u-\nabla_{\tilde{X}_{\ell}}^{A, \mathcal{K}} \nabla_{\tilde{X}_{k}}^{A} u-\nabla_{\nabla_{X_{k}}^{A} X_{\ell}-\nabla_{X_{\ell} X_{k}}^{A}}^{A} u\right)(p) \\
= & \Delta_{A, \mathcal{K}}^{M} u(p)-\left\langle\nabla^{A} u, \mathcal{T}^{\nabla}\right\rangle-\sum_{1 \leq k \leq \ell \leq 3} c_{3}\left(e_{k}\right) c_{3}\left(e_{\ell}\right)\left(v_{F_{A}\left(X_{k}, X_{\ell}\right)}^{S \operatorname{Sin}_{i}^{G}(3)}+\nabla_{T^{\varphi}\left(X_{k}, X_{\ell}\right)}^{A} u\right)(p),
\end{aligned}
$$


where we used $\left\langle\nabla_{X_{k}}^{A} X_{\ell}, X_{m}\right\rangle(p)=-\left\langle X_{\ell}, \nabla_{X_{k}}^{A} X_{m}\right\rangle(p)$ and the second curvature identity in Lemma 6.2.2. The third summand can be reinterpreted as follows:

$$
\begin{aligned}
-\left.\sum_{1 \leq k<\ell \leq 3} c_{3}\left(e_{k}\right) c_{3}\left(E_{\ell}\right) v_{F_{A}\left(X_{k}, X_{\ell}\right)}^{S p i n_{\varepsilon}^{G}(m)}\right|_{u(p)} & =-\sum_{\ell=1}^{3} I_{\ell} v_{* F_{A}\left(X_{\ell}\right)}^{S p i n_{\varepsilon}^{G}(m)}=-\sum_{\ell=1}^{3} I_{\ell} v_{\nu^{-1} * R^{\varphi}\left(X_{\ell}\right)}^{S p(1)}-\sum_{\ell=1}^{3} I_{\ell} v_{* F_{a}\left(X_{\ell}\right)}^{G} \\
& =\left\langle\left.\chi\right|_{u(p)},\left.\nu^{-1} * R^{\varphi}\right|_{p}\right\rangle+\left\langle\mathcal{Y},\left.* F_{a}\right|_{u(p)}\right\rangle . \\
& =\left.\frac{s_{Y}\left(\pi_{Y}(p)\right)}{4} \chi_{0}\right|_{u(p)}+\frac{1}{2}\left\langle\left.\chi_{2}\right|_{u(p)},\left.\operatorname{Ri}_{0}\right|_{p}\right\rangle+\left\langle\left.\mathcal{Y}\right|_{u(p)},\left.* F_{a}\right|_{u(p)}\right\rangle
\end{aligned}
$$

where we used Lemma 6.2.3. Finally, the contribution of the torsion is

$$
-\sum_{1 \leq k \leq \ell \leq 3} c_{3}\left(e_{k}\right) c_{3}\left(e_{\ell}\right) \nabla_{T^{\varphi}\left(X_{k}, X_{\ell}\right)}^{A} u(p)=c_{3}\left(\nabla_{* T^{\varphi}}^{A} u\right)(p) .
$$

\subsection{Lichnerowicz-Weitzenböck formula for the linearized Dirac operator}

We will now prove the Lichnerowicz-Weitzenböck formula for the linearized Dirac operator:

$$
\begin{aligned}
\mathscr{D}_{A}^{l i n, u, *} \mathscr{D}_{A}^{l i n, u} v= & \left.d_{A, \mathcal{K}}^{T M, *} d_{A, \mathcal{K}}^{T M} v+\left.\frac{s_{Y}}{4} \nabla_{v}^{\mathcal{K}}\left(\chi_{0}\right)\right|_{u}+\frac{1}{2}\left\langle\left.\nabla_{v}^{\mathcal{K}}\left(\chi_{2}\right)\right|_{u}, \operatorname{Ric}_{0}\right\rangle+\left\langle\left.\nabla_{v}^{\mathcal{K}}(\mathcal{Y})\right|_{u}, * F_{a}\right\rangle\right) \\
& -c_{3}\left(* \iota_{h o r}^{2} u^{*} F_{\mathcal{K}} v\right)-\left\langle d_{A, \mathcal{K}}^{T M} v, \mathcal{T}^{\varphi}\right\rangle+c_{3}\left(\left\langle d_{A, \mathcal{K}}^{T M} v, * T^{\varphi}\right\rangle\right)-c_{3}\left(\mathcal{T}^{\varphi} \otimes \mathscr{D}_{A}^{l i n, u} v\right) .
\end{aligned}
$$

Proof (Theorem 6.2.1, part 2). We use the same notation as in the proof of the first part of Theorem 6.2.1. From the first curvature identity in Lemma 6.2.2 we obtain

$$
\begin{aligned}
\mathscr{D}_{A}^{l i n, u} \mathscr{D}_{A}^{l i n, u} v(p)= & \sum_{k=1}^{3} \sum_{\ell=1}^{3} c_{3}\left(e_{k}\right) \nabla_{\tilde{X}_{k}}^{A, \mathcal{K}}\left(c_{3}\left(f_{\ell}\right) \nabla_{\tilde{X}_{\ell}}^{A, \mathcal{K}} v\right)(p) \\
= & \sum_{k=1}^{3} \sum_{\ell=1}^{3}\left(c_{3}\left(e_{k}\right) c_{3}\left(e_{\ell}\right) \nabla_{\tilde{X}_{k}}^{A, \mathcal{K}} \nabla_{\tilde{X}_{\ell}}^{A, \mathcal{K}} v-c_{3}\left(e_{k}\right) c_{3}\left(e_{\ell}\right) \nabla_{\nabla_{X_{k}}^{A} X_{\ell}}^{A, \mathcal{K}} v\right)(p) \\
= & -\operatorname{tr}\left(d_{A, \mathcal{K}}^{\mathbb{R}^{3} \otimes T}\left(d_{A, \mathcal{K}}^{T M} v\right)\right)(p) \\
& +\sum_{3 \leq \ell} c_{3}\left(e_{k}\right) c_{3}\left(e_{\ell}\right)\left(\nabla_{\tilde{X}_{k}}^{A, \mathcal{K}} \nabla_{\tilde{X}_{\ell}}^{A, \mathcal{K}} v-\nabla_{\tilde{X}_{\ell}}^{A, \mathcal{K}} \nabla_{\tilde{X}_{k}}^{A, \mathcal{K}} v-\nabla_{\nabla_{X_{k}}^{A} X_{\ell}-\nabla_{X_{\ell}}^{A} X_{k}}^{A} v\right)(p) \\
& 1 \leq k \leq \ell \leq 3 \\
& d_{A, \mathcal{K}}^{T M, *} d_{A, \mathcal{K}}^{T M} v(p)-\left\langle d_{A, \mathcal{K}}^{T M} v, \mathcal{T}^{\varphi}\right\rangle(p) \\
& +\sum c_{3}\left(e_{k}\right) c_{3}\left(e_{\ell}\right)\left(F_{\mathcal{K}}\left(\nabla_{\tilde{X}_{k}}^{A} u, \nabla_{\tilde{X}_{\ell}}^{A} u\right) v-\mathcal{K}\left(\left.v_{F_{A}\left(X_{k}, X_{\ell}\right)}^{G}\right|_{v}\right)-\nabla_{T^{\varphi}\left(X_{k}, X_{\ell}\right)}^{A, \mathcal{K}} v\right)(p) . \\
& 1 \leq k \leq \ell \leq 3
\end{aligned}
$$

We first compute

$$
\begin{aligned}
& \sum_{k<\ell} c\left(e_{k}\right) c\left(e_{\ell}\right) F_{\mathcal{K}}\left(T u\left(\tilde{X}_{k}\right), T u\left(\tilde{X}_{\ell}\right)\right) v(p) \\
= & \sum_{\ell=1}^{3} I_{\ell}\left(* F_{\mathcal{K}}\right)\left(T u\left(\tilde{X}_{\ell}\right)\right) v(p)=\sum_{\ell=1}^{3} I_{\ell}\left(* u^{*} F_{\mathcal{K}}\right)\left(\tilde{X}_{\ell}\right) v(p) \\
= & -c_{3}\left(* \iota_{h o r}^{2} u^{*} F_{\mathcal{K}} v(p)\right) .
\end{aligned}
$$


For the other curvature term we use $\left.\mathcal{K} v_{\xi}^{S p i n_{\varepsilon}^{G}(3)}\right|_{v(p)}=\left.\left(\nabla_{v(p)}^{\mathcal{K}} v_{\xi}^{S p i n_{\varepsilon}^{G}(3)}\right)\right|_{u(p)}$ :

$$
\begin{aligned}
-\left.\sum_{k<\ell} c\left(e_{k}\right) c\left(e_{\ell}\right) \mathcal{K} v_{F_{A}\left(\tilde{X}_{k}, \tilde{X}_{\ell}\right)}^{\operatorname{Spin}_{G}^{G}(3)}\right|_{v(p)}= & -\left.\sum_{\ell=1}^{3} I_{\ell} \mathcal{K} v_{* F_{A}\left(\tilde{X}_{\ell}\right)}^{\operatorname{Spin}_{\varepsilon}^{G}(3)}\right|_{v(p)}=-\left.\sum_{\ell=1}^{3} I_{\ell} \nabla_{v(p)}^{\mathcal{K}}\left(v_{* F_{A}\left(\tilde{X}_{\ell}\right)}^{\operatorname{Spin}_{\varepsilon}^{G}(3)}\right)\right|_{u(p)} \\
= & -\left.\sum_{\ell=1}^{3} \nabla_{v(p)}^{\mathcal{K}}\left(I_{\ell} v_{* F_{A}\left(\tilde{X}_{\ell}\right)}^{\operatorname{Spin}_{\varepsilon}^{G}(3)}\right)\right|_{u(p)} \\
= & \left.\nabla_{v(p)}^{\mathcal{K}}\left(\left\langle\chi,\left.\nu^{-1} * R^{\varphi}\right|_{p}\right\rangle+\left\langle\mathcal{Y},\left.* F_{a}\right|_{p}\right\rangle\right)\right|_{u(p)} \\
= & \nabla_{v(p)}^{\mathcal{K}}\left(\left.\left\langle\frac{s_{Y}\left(\pi_{Y}(p)\right)}{4} \chi_{0}+\frac{1}{2}\left\langle\chi_{2},\left.\operatorname{Ric}_{0}\right|_{p}\right\rangle+\left\langle\mathcal{Y}, * F_{a}\right\rangle\right)\right|_{u(p)}\right. \\
= & \left.\frac{s_{Y}\left(\pi_{Y}(p)\right)}{4} \nabla_{v(p)}^{\mathcal{K}}\left(\chi_{0}\right)\right|_{u(p)}+\frac{1}{2}\left\langle\left.\nabla_{v(p)}^{\mathcal{K}}\left(\chi_{2}\right)\right|_{u(p)},\left.\operatorname{Ric}_{0}\right|_{p}\right\rangle \\
& +\left\langle\left.\nabla_{v(p)}^{\mathcal{K}}(\mathcal{Y})\right|_{u(p)},\left.* F_{a}\right|_{u(p)}\right\rangle .
\end{aligned}
$$

Finally, the contribution of the torsion is

$$
-\sum_{k \leq \ell} c_{3}\left(e_{k}\right) c_{3}\left(e_{\ell}\right) \nabla_{T^{\varphi}\left(\tilde{X}_{k}, \tilde{X}_{\ell}\right)}^{A, \mathcal{L}} v(p)=c_{3}\left(\nabla_{* T^{\varphi}}^{A, \mathcal{K}} v\right)(p)
$$

\section{5 $\quad L^{2}$-Lichnerowicz-Weitzenböck formula}

We will now prove the final Lichnerowicz-Weitzenböck formula, which compares the norms of the Dirac operator and the covariant derivative:

$$
\begin{aligned}
\left\|\mathscr{D}_{A} u\right\|^{2}= & \left\|d_{A}^{M} u\right\|^{2}-2\left\langle\Phi_{3}(u), * F_{a}\right\rangle+\frac{s_{Y}}{2} \rho_{0} \circ u+\left\langle\rho_{2} \circ u, \text { Ric }_{0}\right\rangle \\
& +2 * d\left\langle\theta_{Y} \wedge\left(u^{*} \gamma\right)_{h o r}\right\rangle-2 *\left\langle T^{\varphi} \wedge\left(u^{*} \gamma\right)_{h o r}\right\rangle .
\end{aligned}
$$

The 4-dimensional version of this formula can be found in [Pid04]. Our approach is similar to the proof in [Pid04], however avoids using the frame bundle of the hyperkähler manifold $M$.

Proof (Theorem 6.2.1, part 3). First consider the H-valued form $h^{M}=g^{M}+i \omega_{1}+j \omega_{2}+$ $k \omega_{3} \in \mathbb{H} \otimes \Gamma\left(M, T^{*} M \otimes T^{*} M\right)$. Let $x, x^{\prime} \in \mathbb{R}^{3} \cong \operatorname{Im}(\mathbb{H})$ and $v, v^{\prime} \in T_{x} M$ and note that $h^{M}$ is H-linear in the following sense:

$$
h^{M}\left(\mathcal{I}_{x} v, v^{\prime}\right)=x h^{M}(v, w) \text { and } h^{M}\left(v, \mathcal{I}_{x^{\prime}} v^{\prime}\right)=h^{M}(v, w) \bar{x}^{\prime} .
$$

The induced metric on $\mathbb{R}^{3} \otimes T M$ is

$$
\left\langle x \otimes v, x^{\prime} \otimes v^{\prime}\right\rangle_{\mathbb{R}^{3} \otimes T M}=\operatorname{Re}\left(x^{\prime *} x\right) g^{M}\left(v, v^{\prime}\right)=\operatorname{Re}\left(x^{\prime *} x\right) \operatorname{Re}\left(h^{M}\left(v, v^{\prime}\right)\right) .
$$

Furthermore,

$$
\begin{aligned}
g^{M}\left(c_{3}(x \otimes v), c_{3}\left(x^{\prime} \otimes v\right)\right) & =\operatorname{Re}\left(h^{M}\left(\mathcal{I}_{\bar{x}} v, \mathcal{I}_{\bar{x}^{\prime}} v^{\prime}\right)\right)=\operatorname{Re}\left(\bar{x} h^{M}\left(v, v^{\prime}\right) x^{\prime}\right)=\operatorname{Re}\left(x^{\prime} \bar{x} h^{M}\left(v, v^{\prime}\right)\right) \\
& =\operatorname{Re}\left(x^{\prime} \bar{x}\right) \operatorname{Re}\left(h^{M}\left(v, v^{\prime}\right)\right)+\operatorname{Re}\left(\operatorname{Im}\left(x^{\prime} \bar{x}\right) \operatorname{Im}\left(h^{M}\left(v, v^{\prime}\right)\right)\right) \\
& =\left\langle x \otimes v, x^{\prime} \otimes v^{\prime}\right\rangle_{\mathbb{R}^{3} \otimes T M}-\left\langle\operatorname{Im}\left(x^{\prime} \bar{x}\right), \omega\left(v, v^{\prime}\right)\right\rangle .
\end{aligned}
$$


Therefore,

$$
\begin{aligned}
g^{M}\left(\mathscr{D}_{A} u, \mathscr{D}_{A} u\right) & =\left\langle d_{A}^{M} u, d_{A}^{M} u\right\rangle_{\mathbb{R}^{3} \otimes T M}-\sum_{k, \ell=1}^{3}\left\langle\operatorname{Im}\left(\zeta_{\ell} \bar{\zeta}_{k}\right), \omega\left(T u\left(\tilde{e}_{k}\right), T u\left(\tilde{e}_{\ell}\right)\right)\right\rangle \\
& =\left\langle d_{A}^{M} u, d_{A}^{M} u\right\rangle_{\mathbb{R}^{3} \otimes T M}-2 \sum_{k<\ell}\left\langle\zeta_{k} \zeta_{\ell}, u^{*} \omega\left(\tilde{e}_{k}, \tilde{e}_{\ell}\right)\right\rangle \\
& =\left\langle d_{A}^{M} u, d_{A}^{M} u\right\rangle_{\mathbb{R}^{3} \otimes T M}-2 *\left\langle\theta_{Y} \wedge\left(u^{*} \omega\right)_{h o r}\right\rangle,
\end{aligned}
$$

where $\theta_{Y}$ is the canonical 1-form $\theta_{Y} \in \Omega^{1}\left(Q_{3}, \mathbb{R}^{3}\right)^{\operatorname{Spin}_{\varepsilon}^{G}(3)}$ and $\left(u^{*} \omega\right)_{h o r}$ denotes the composition of the horizontal projection for $A$ and $u^{*} \omega$. Since $\omega=d \gamma$ and $\left(u^{*} \omega\right)_{h o r}=$ $\left(d u^{*} \gamma\right)_{h o r}=d_{A}\left(u^{*} \gamma\right)_{h o r}+u^{*} \gamma\left(v_{F_{A}}^{S_{\varepsilon}}{ }^{G}(3)\right)($ cf. [Pid04, Lem. 5.3]), we have

$$
2\left\langle\theta_{Y} \wedge\left(u^{*} \omega\right)_{h o r}\right\rangle=-2 d\left\langle\theta_{Y} \wedge\left(u^{*} \gamma\right)_{h o r}\right\rangle+2\left\langle d_{A} \theta_{Y} \wedge\left(u^{*} \gamma\right)_{h o r}\right\rangle+2\left\langle\theta_{Y} \wedge u^{*} \gamma\left(v_{F_{A}}^{S p i n_{\varepsilon}^{G}(3)}\right)\right\rangle .
$$

Since $u: Q \rightarrow M$ is $\operatorname{Spin}_{\varepsilon}^{G}(3)$-equivariant and $\iota_{\mathfrak{s p i n}_{\varepsilon}^{G}(3)} \gamma=\mu-\rho$, we obtain

$$
u^{*} \gamma\left(v_{F_{A}}^{\operatorname{Spin}_{\varepsilon}^{G}(3)}\right)=\left\langle u^{*} \iota_{\mathfrak{s p i n}_{\varepsilon}^{G}(3)} \gamma, F_{A}\right\rangle=\left\langle u^{*}(\mu-\rho), F_{A}\right\rangle
$$

and hence

$$
\begin{aligned}
& \left\langle\theta_{Y} \wedge\left(u^{*} \omega\right)_{h o r}\right\rangle \\
= & -d\left\langle\theta_{Y} \wedge\left(u^{*} \gamma\right)_{h o r}\right\rangle+\left\langle T^{\varphi} \wedge\left(u^{*} \gamma\right)_{h o r}\right\rangle+\left\langle\theta_{Y} \wedge u^{*} \mu\left(F_{a}\right)\right\rangle-\left\langle\theta_{Y} \wedge u^{*} \rho\left(F_{\varphi}\right)\right\rangle \\
= & -d\left\langle\theta_{Y} \wedge\left(u^{*} \gamma\right)_{h o r}\right\rangle+\left\langle T^{\varphi} \wedge\left(u^{*} \gamma\right)_{h o r}\right\rangle+\left\langle\mu \circ u, * F_{a}\right\rangle * 1-\left\langle\rho \circ u, \nu^{-1} * R^{\varphi}\right\rangle * 1 \\
= & -d\left\langle\theta_{Y} \wedge\left(u^{*} \gamma\right)_{h o r}\right\rangle+\left\langle T^{\varphi} \wedge\left(u^{*} \gamma\right)_{h o r}\right\rangle+\left(\left\langle\Phi_{3}(u), * F_{a}\right\rangle-\frac{s_{Y}}{4} \rho_{0} \circ u-\frac{1}{2}\left\langle\rho_{2} \circ u, R_{i}\right\rangle\right) * 1 .
\end{aligned}
$$

In particular,

$$
\begin{aligned}
\left\|\mathscr{D}_{A} u\right\|^{2}= & \left\|d_{A}^{M} u\right\|^{2}+2 * d\left\langle\theta_{Y} \wedge\left(u^{*} \gamma\right)_{h o r}\right\rangle-2 *\left\langle T^{\varphi} \wedge\left(u^{*} \gamma\right)_{h o r}\right\rangle \\
& -2\left\langle\Phi_{3}(u), * F_{a}\right\rangle+\frac{s_{Y}}{2} \rho_{0} \circ u+\left\langle\rho_{2} \circ u, R i c_{0}\right\rangle .
\end{aligned}
$$

If $u \in C^{\infty}(Q, M)^{\operatorname{Spin}_{\varepsilon}^{G}(3)}$ has compact support, we can integrate over $Y$ and obtain

$$
\begin{aligned}
\left\|\mathscr{D}_{A} u\right\|_{L^{2}}^{2}= & \left\|d_{A}^{M} u\right\|_{L^{2}}^{2}-2\left\langle\Phi_{3}(u), * F_{a}\right\rangle_{L^{2}}+\int_{Y} \frac{s_{Y}}{2} \rho_{0} \circ u+\int_{Y}\left\langle\rho_{2} \circ u, R_{i c_{0}}\right\rangle \\
& +2 \int_{\partial Y}\left\langle\theta_{Y} \wedge\left(u^{*} \gamma\right)_{h o r}\right\rangle-2 \int_{Y}\left\langle T^{\varphi} \wedge\left(u^{*} \gamma\right)_{h o r}\right\rangle
\end{aligned}
$$

\subsection{Seiberg-Witten functional}

It follows immediately from the Lichnerowicz-Weitzenböck formula that, on a compact oriented Riemannian 3-manifold $Y$, the solutions of the Seiberg-Witten equations are the 
zeros of the functional

$$
\begin{aligned}
L_{S W}(u, A):= & \left\|* F_{a}+\Phi_{3}(u)\right\|_{L^{2}}^{2}+\left\|\mathscr{D}_{A} u\right\|_{L^{2}}^{2} \\
= & \left\|F_{a}\right\|_{L^{2}}^{2}+2\left\langle * F_{a}, \Phi_{3}(u)\right\rangle_{L^{2}}+\left\|\Phi_{3}(u)\right\|_{L^{2}}^{2}+\left\|d_{A}^{M} u\right\|_{L^{2}}^{2} \\
& +\frac{1}{2}\left\langle s_{Y}, \rho_{0} \circ u\right\rangle_{L^{2}}+\left\langle R i c_{0}, \rho_{2} \circ u\right\rangle_{L^{2}}-2\left\langle\Phi_{3}(u), * F_{a}\right\rangle_{L^{2}} \\
& -2\left\langle T^{\nabla} \wedge\left(u^{*} \gamma\right)_{h o r}\right\rangle_{L^{2}}+2 \int_{\partial Y}\left\langle\theta_{Y} \wedge\left(u^{*} \gamma\right)_{h o r}\right\rangle \\
= & \left\|F_{a}\right\|_{L^{2}}^{2}+\left\|\Phi_{3}(u)\right\|_{L^{2}}^{2}+\left\|d_{A}^{M} u\right\|_{L^{2}}^{2}+\frac{1}{2}\left\langle s_{Y}, \rho_{0} \circ u\right\rangle_{L^{2}}+\left\langle R i c_{0}, \rho_{2} \circ u\right\rangle_{L^{2}} \\
& -2\left\langle T^{\nabla} \wedge\left(u^{*} \gamma\right)_{h o r}\right\rangle_{L^{2}}+2 \int_{\partial Y}\left\langle\theta_{Y} \wedge\left(u^{*} \gamma\right)_{h o r}\right\rangle .
\end{aligned}
$$

In particular, if $\chi_{2}=0, s_{Y} \geq 0, T^{\nabla}=0$ and $\partial Y=\emptyset$, then $L_{S W} \geq 0$ and a solution satisfies $F_{a}=0, d_{A} u=0$, and either $\rho_{0} \circ u=0$ or $s_{Y}=0$.

\subsection{Lichnerowicz-Weitzenböck formulae in dimension 4}

The 4-dimensional version of Theorem 6.2.1, reads

6.7.1 Theorem ([Sch10, Thm. 4.7.1, Thm. 4.7.2], [Pid04, Thm. 5.4]).

Let $Q_{4} \rightarrow X$ be a $\operatorname{Spin}_{\varepsilon}^{G}(4)$-structure on an oriented Riemannian 4-manifold $X, u \in$ $C^{\infty}\left(Q_{4}, M\right)^{\operatorname{Spin}_{\varepsilon}^{G}(4)}$ a spinor, $v \in C^{\infty}\left(Q_{4}, T M\right)^{\operatorname{Spin}_{\varepsilon}^{G}(4)}$ satisfying $\pi_{M} \circ v=u$ and $A \in \mathscr{A}_{4}$ lifting $\varphi$. Let $\eta_{\ell}=\left(e_{0} \wedge e_{\ell}\right)_{+} \in \bigwedge_{+}^{2} \mathbb{R}^{4}$ Then

1. Lichnerowicz-Weitzenböck formula for the generalized Dirac operator:

$$
\begin{aligned}
\mathscr{D}_{A}^{l i n, u,+, *} \mathscr{D}_{A}^{+} u= & \Delta_{A, \mathcal{K}}^{M} u-\left\langle d_{A}^{M} u, \mathcal{T}^{\varphi}\right\rangle+\left.\frac{s X}{4} \chi_{0}\right|_{u}+\frac{1}{2}\left\langle\left.\chi_{2}\right|_{u}, R_{X, 0}^{++}\right\rangle+\left\langle\left.\mathcal{Y}\right|_{u}, \frac{1}{2} R_{X}^{+-}+F_{a}^{+}\right\rangle \\
& +c_{4}\left(\left\langle T^{\varphi,+}, d_{A}^{M} u\right\rangle\right)-c_{4}\left(\mathcal{T}^{\varphi} \otimes \mathscr{D}_{A} u\right) .
\end{aligned}
$$

2. Lichnerowicz-Weitzenböck formula for linearized Dirac operator:

$$
\begin{aligned}
\mathscr{D}_{A}^{l i n, u,+, *} \mathscr{D}_{A}^{l i n, u,+} v= & d_{A, \mathcal{K}}^{T M, *} d_{A, \mathcal{K}}^{T M} v+\left.\frac{s_{X}}{4} \nabla_{v}^{\mathcal{K}}\left(\chi_{0}\right)\right|_{u}+\frac{1}{2}\left\langle\left.\nabla_{v}^{\mathcal{K}}\left(\chi_{2}\right)\right|_{u}, R_{X, 0}^{++}\right\rangle \\
& +\left\langle\left.\nabla_{v}^{\mathcal{K}}(\mathcal{Y})\right|_{u}, \frac{1}{2} R_{X}^{+-}+F_{a}^{+}\right\rangle+2 \sum_{\ell=1}^{3} I_{\ell}\left\langle\left(u^{*} F_{\mathcal{K}}\right)_{h o r}, \eta_{\ell}\right\rangle v \\
& -\left\langle d_{A, \mathcal{K}}^{T M} v, \mathcal{T}^{\varphi}\right\rangle+c_{4}\left(\left\langle d_{A, \mathcal{K}}^{T M} v, T^{\varphi,+}\right\rangle\right)-c_{4}\left(\mathcal{T}^{\varphi} \otimes \mathscr{D}_{A}^{l i n, u} v\right)
\end{aligned}
$$

3. Norms and $L^{2}$-Lichnerowicz-Weitzenböck formula:

$$
\begin{aligned}
\left\|\mathscr{D}_{A}^{+} u\right\|^{2}= & \left\|d_{A}^{M} u\right\|^{2}+\frac{s_{X}}{2} \rho_{0} \circ u+\left\langle R_{X, 0}^{++}, \rho_{2} \circ u\right\rangle-\left\langle R_{X, 0}^{+-}, \mu^{S p(1)_{-}} \circ u\right\rangle-2\left\langle\Phi_{4}(u), F_{a}^{+}\right\rangle \\
& -* d\left\langle\left(u^{*} \gamma\right)_{h o r} \wedge\left(\theta_{X} \wedge \theta_{X}\right)_{+}\right\rangle+\left\langle\left(u^{*} \gamma\right)_{h o r} \wedge\left(T^{\varphi} \wedge \theta_{X}-\theta_{X} \wedge T^{\varphi}\right)_{+}\right\rangle
\end{aligned}
$$


In particular, if $u \in C^{\infty}\left(Q_{4}, M\right)^{\operatorname{Spin}_{\varepsilon}^{G}(4)}$ has compact support, we can integrate over $X$ and obtain

$$
\begin{aligned}
\left\|\mathscr{D}_{A}^{+} u\right\|_{L^{2}}^{2}= & \left\|d_{A}^{M} u\right\|_{L^{2}}^{2}-2\left\langle\Phi_{4}(u), F_{a}^{+}\right\rangle_{L^{2}} \\
& +\int_{X} \frac{s X}{2} \rho_{0} \circ u+\int_{X}\left\langle R_{X, 0}^{++}, \rho_{2} \circ u\right\rangle-\int_{X}\left\langle R_{X}^{+-}, \mu^{S p(1)_{-}} \circ u\right\rangle \\
& -\int_{\partial X}\left\langle\left(u^{*} \gamma\right)_{h o r} \wedge\left(\theta_{X} \wedge \theta_{X}\right)_{+}\right\rangle+\int_{X}\left\langle\left(u^{*} \gamma\right)_{h o r} \wedge\left(T^{\varphi} \wedge \theta_{X}-\theta_{X} \wedge T^{\varphi}\right)_{+}\right\rangle .
\end{aligned}
$$

Here, $R_{X, 0}^{++}$is the positive Weyl curvature and $R_{X}^{+-} \in C^{\infty}\left(Q_{4}, \bigwedge_{+}^{2}\left(\mathbb{R}^{4}\right)^{\vee} \otimes \bigwedge_{-}^{2} \mathbb{R}^{4}\right)^{S p i n} n_{\varepsilon}^{G}(4)$ is component of the Riemannian curvature tensor $R \in C^{\infty}\left(Q_{4}, \bigwedge^{2}\left(\mathbb{R}^{4}\right)^{\vee} \otimes \wedge^{2} \mathbb{R}^{4}\right)^{\operatorname{Spin}_{\varepsilon}^{G}(4)}$ of $\varphi$. Note that $R_{X}^{+-}$can be identified with the traceless part of the Ricci curvature.

6.7.2 Remark. The proofs for these formulae in the case when $\varphi$ is the Levi-Civita connection and $S p(1)_{-}$acts trivially, can be found in [Sch10, Thm. 4.7.1, Thm. 4.7.2], [Pid04, Thm. 5.4]. The proof in the case of non-vanishing torsion is similar to 3-dimensional case. In the case of a non-trivial $S p(1)_{-}$-action, the fundamental vector field for the $S p(1)_{-}$-action appears in addition to the fundamental vector field for the $S p(1)_{+}$-action and leads to the additional terms containing $R_{X}^{+-}$.

6.7.3 Remark. If $M$ admits a $\operatorname{Spin}_{\varepsilon}^{G}(4)$-invariant vector field $\hat{\chi}$ which satisfies $\nabla^{\mathcal{K}} \hat{\chi}=$ $\operatorname{id}_{T M}$, then a $L^{2}$-Weitzenböck formula also follows from the first part of Proposition 4.5.10, Theorem 6.7.1 and Lemma 4.5.12:

$$
\begin{aligned}
\left\|\mathscr{D}_{A}^{+} u\right\|_{L^{2}}^{2}= & \left\langle\hat{\chi}_{u}, \mathscr{D}_{A}^{l i n, u,+, *} \mathscr{D}_{A}^{+} u\right\rangle_{L^{2}}-\int_{\partial X} \pi_{!} g\left(\hat{\chi}_{u}, c_{4}\left(f_{\overrightarrow{\mathfrak{n}}} \otimes \mathscr{D}_{A}^{+} u\right)\right) \\
= & \left\langle\hat{\chi}_{u}, \Delta_{A, \mathcal{K}}^{M} u-\left\langle d_{A}^{M} u, \mathcal{T}^{\varphi}\right\rangle+\left.\frac{s_{X}}{4} \chi_{0}\right|_{u}+\frac{1}{2}\left\langle\left.\chi_{2}\right|_{u}, R_{X, 0}^{++}\right\rangle+\left\langle\left.\mathcal{Y}\right|_{u}, \frac{1}{2} R_{X}^{+-}+F_{a}^{+}\right\rangle\right\rangle_{L^{2}} \\
& +\left\langle\hat{\chi}_{u}, c_{4}\left(\left\langle T^{\varphi,+}, d_{A}^{M} u\right\rangle\right)-c_{4}\left(\mathcal{T}^{\varphi} \otimes \mathscr{D}_{A}^{+} u\right)\right\rangle_{L^{2}}-\int_{\partial X} \pi_{!} g\left(\hat{\chi}_{u}, c_{4}\left(f_{\overrightarrow{\mathfrak{n}}} \otimes \mathscr{D}_{A}^{+} u\right)\right) \\
= & \left\|d_{A}^{M} u\right\|_{L^{2}}^{2}-\left\langle\hat{\chi}_{u},\left\langle d_{A}^{M} u, \mathcal{T}^{\varphi}\right\rangle+\left.\frac{s_{X}}{4} \chi_{0}\right|_{u}+\frac{1}{2}\left\langle\left.\chi_{2}\right|_{u}, R_{X, 0}^{++}\right\rangle+\left\langle\left.\mathcal{Y}\right|_{u}, \frac{1}{2} R_{X}^{+-}+F_{a}^{+}\right\rangle\right\rangle_{L^{2}} \\
& +\left\langle\hat{\chi}_{u}, c_{4}\left(\left\langle T^{\varphi,+}, d_{A}^{M} u\right\rangle\right)-c_{4}\left(\mathcal{T}^{\varphi} \otimes \mathscr{D}_{A}^{+} u\right)\right\rangle_{L^{2}}-\int_{\partial X} \pi_{!} g\left(\hat{\chi}_{u}, c_{4}\left(f_{\overrightarrow{\mathfrak{n}}} \otimes \mathscr{D}_{A}^{+} u\right)\right) \\
& -\int_{\partial X} \pi_{!} g\left(\hat{\chi}_{u_{\partial X}}, d_{A}^{M} u\left(f_{\overrightarrow{\mathfrak{n}}}\right)\right) \\
= & \left\|d_{A}^{M} u\right\|_{L^{2}}^{2}-\left\langle\hat{\chi}_{u},\left\langle d_{A}^{M} u, \mathcal{T}^{\varphi}\right\rangle+\left.\frac{s_{X}}{4} \chi_{0}\right|_{u}+\frac{1}{2}\left\langle\left.\chi_{2}\right|_{u}, R_{X, 0}^{++}\right\rangle+\left\langle\left.\mathcal{Y}\right|_{u}, \frac{1}{2} R_{X}^{+-}+F_{a}^{+}\right\rangle\right\rangle_{L^{2}} \\
& \left.\left.+\left\langle\hat{\chi}_{u}, c_{4}\left(\left\langle T^{\varphi,+}, d_{A}^{M} u\right\rangle\right)-c_{4}\left(\mathcal{T}^{\varphi} \otimes \mathscr{D}_{A}^{+} u\right)\right\rangle\right\rangle_{L^{2}}+\int_{\partial X} \pi_{!} g\left(\hat{\chi}_{u_{\partial X}}, \mathscr{D}_{A^{\partial X}} u_{\partial X}\right)\right) \\
& -\int_{\partial X} \pi_{!} g\left(\hat{\chi}_{u_{\partial X}},\left.\frac{H}{2}\left(\chi_{0}+\chi_{0}^{-}\right)\right|_{u_{\partial X}}\right) \\
& +\int_{\partial X} \pi_{!} g\left(\hat{\chi}_{u_{\partial X}}, \frac{1}{2}\left\langle N_{0},\left.\left(\chi_{2}+\chi_{2}^{-}\right)\right|_{u_{\partial X}}\right\rangle+\frac{1}{2}\left\langle g\left(\overrightarrow{\mathfrak{n}}, * T^{\varphi}\right),\left.\left(\chi_{A l t}+\chi_{A l t}^{-}\right)\right|_{u_{\partial X}}\right\rangle\right),
\end{aligned}
$$

where we use the same notation as in Lemma 4.5.12. If we restrict ourselves to the case where $\rho_{2}=0$ and $\hat{\chi}=\chi_{0}$, the chosen connection on $P_{S O(4)}$ is the Levi-Civita connection, and $S p(1)_{-}$acts trivially on $M$, then this reads

$$
\begin{aligned}
\left\|\mathscr{D}_{A}^{+} u\right\|_{L^{2}}^{2}= & \left\|d_{A}^{M} u\right\|_{L^{2}}^{2}+\left\langle\left.\chi_{0}\right|_{u},\left.\frac{s_{X}}{4} \chi_{0}\right|_{u}\right\rangle_{L^{2}}-2\left\langle\Phi_{4}(u), F_{a}^{+}\right\rangle_{L^{2}} \\
& \left.+\int_{\partial X} \pi_{!} g\left(\left.\chi_{0}\right|_{u_{\partial X}}, \mathscr{D}_{A} \partial u_{\partial X}\right)\right)-\int_{\partial X} \pi_{!} g\left(\left.\chi_{0}\right|_{u_{\partial X}},\left.\frac{H}{2} \chi_{0}\right|_{u_{\partial X}}\right)
\end{aligned}
$$


In particular, we have the topological energy and the analytic energy defined as follows

$$
\begin{aligned}
\mathcal{E}^{t o p}(u, A) & :=-\frac{1}{2}\left\|\left\langle F_{a} \wedge F_{a}\right\rangle_{\mathfrak{g}}\right\|_{L^{2}}^{2}-\int_{\partial X}\left\langle\left.\chi_{0}\right|_{u}, \mathscr{D}_{A^{\partial X}}\left(u_{\partial X}\right)\right\rangle+\frac{1}{2} \int_{\partial X}\left\langle\left.\chi_{0}\right|_{u_{\partial X}},\left.H \chi_{0}\right|_{u_{\partial X}}\right\rangle, \\
\mathcal{E}^{a n}(u, A) & :=\frac{1}{2}\left\|F_{a}\right\|_{L^{2}}^{2}+\left\|d_{A}^{M} u\right\|^{2}+\left\langle\left.\chi_{0}\right|_{u},\left.\frac{s_{X}}{4} \chi_{0}\right|_{u}\right\rangle_{L^{2}}+\left\|\Phi_{4}(u)\right\|_{L^{2}}^{2} .
\end{aligned}
$$

Since in this situation

$$
\mathcal{E}^{a n}(u, A)=\mathcal{E}^{t o p}(u, A)+\left\|\mathscr{D}_{A}^{+} u\right\|_{L^{2}}^{2}+\left\|F_{a}^{+}+\Phi_{4}(u)\right\|_{L^{2}}^{2},
$$

solutions of the generalized Seiberg-Witten equations have a well-defined energy. This geralizes the case of the Seiberg-Witten equations (cf. [KM07, Section 4]).

Note that in this situation, there is also a Chern-Simons-Dirac functional ([Cal10]), which is closely related to the topological energy.

However, also other situations (for example nontrivial torsion), it can be interesting to study the corresponding energies. Examples are the Fueter operator constructed from a divergence-free frame ([Sal13]) and the Vafa-Witten equations (where $\rho_{2} \not \equiv 0$ ).

In the general case, it is however unknown ho the boundary terms are related to the Dirac operator on the boundary. 


\section{Chapter 7}

\section{Conclusion}

We have seen how the differential forms which naturally appear on a hyperkähler manifold with permuting $\operatorname{Spin}_{\varepsilon}^{G}(m)$-action can be interpreted in terms of the Cartan model for equivariant cohomology. The cocycles constructed from these then give rise to homotopy moment maps. More generally, we provided a natural construction of a homotopy moment map for each cocycle in the Cartan model for equivariant cohomology, generalizing the construction of Atiyah and Bott ([AB84]).

One of the applications of moment maps in symplectic geometry is the symplectic reduction. While it is still unknown what a general multisymplectic reduction is, there are examples for which one can perform such a reduction ([CFRZ15]). Also in the cases of tri-hamiltonian action on hyperkähler manifolds and hamiltonian actions on quaternionic Kähler manifolds, a "reduction" can easily be constructed, which recovers the notion of hyperkähler/quaternionic Kähler quotients. However, in all these examples, the components of the moment maps are either determined by $f_{1}$, or many of the components vanish. Examples are tri-hamiltonian actions for $G=S^{1}$, where only $f_{1}$ is non-zero and, on the other hand, permuting action on Swann bundles, or more generally the reductions constructed by Madsen and Swann [MS12], [MS13], where only the highest component $f_{n}$ is used to construct the quotient. It is therefore unknown how a reduction should be constructed in the general case.

Hyperkähler manifolds with permuting $\operatorname{Spin}_{\varepsilon}^{G}(m)$-action are also a crucial ingredient for the generalized Seiberg-Witten equations, where such a manifold takes the role of the spinor representation in Seiberg-Witten theory. Starting with the anti-selfduality equations and Donaldson theory, later Seiberg-Witten theory, the generalized SeibergWitten equations for various target manifolds had a great impact on low-dimensional geometry and topology. More recently, other examples turned out to be closely related to gauge theories in dimensions 5, 6, 7 and 8 ([Hay12], [Hay15b], [DS11]). A uniform treatment of all these cases would be desirable, as well as, ultimately, a Floer theory for generalized Seiberg-Witten theory. While the Lichnerowicz-Weitzenböck formulae in dimension three provide another step in this direction, this goal is currently beyond reach, as the properties of the moduli spaces (in particular compactness or compactifications) are not yet understood well enough. However, the progress made in the case of complex antiselfduality equations ([Tau13b], [Tau13a], [Tau14]) as well in the case of Seiberg-Witten 
equations with $n$ spinors ([HW15]) and also in the case of Fueter sections ([Wal15]), might ultimately lead to a better understanding of the moduli spaces for generalized Seiberg-Witten equations constructed from a larger class of hyperkähler manifolds. The bubbling phenomenon in codimension 2, which can be seen in these cases, is analytically involved and not yet completely understood. However, there is hope that this leads to a suitable compactification of the moduli spaces. 


\section{Bibliography}

[AB84] Michael F. Atiyah and Raoul Bott, The moment map and equivariant cohomology, Topology 23 (1984), no. 1, 1-28.

[AH88] Michael Atiyah and Nigel Hitchin, The geometry and dynamics of magnetic monopoles, M. B. Porter Lectures, Princeton University Press, Princeton, NJ, 1988.

[AHDM78] Michael. F. Atiyah, Nigel J. Hitchin, Vladimir. G. Drinfel'd, and Yuri I. Manin, Construction of instantons, Phys. Lett. A 65 (1978), no. 3, 185-187.

[BD00] Roger Bielawski and Andrew S. Dancer, The geometry and topology of toric hyperkähler manifolds, Comm. Anal. Geom. 8 (2000), no. 4, 727-760.

[BGM93] Charles P. Boyer, Krzysztof Galicki, and Benjamin M. Mann, Quaternionic reduction and Einstein manifolds, Comm. Anal. Geom. 1 (1993), no. 2, 229279 .

[Bie97] Roger Bielawski, Hyper-Kähler structures and group actions, J. London Math. Soc. (2) 55 (1997), no. 2, 400-414.

[Bie99]__ Twistor quotients of hyperkähler manifolds, Quaternionic structures in mathematics and physics (Rome, 1999), Univ. Studi Roma "La Sapienza", Rome, 1999, pp. 7-21 (electronic).

[Bie07]_L Lie groups, Nahm's equations and hyperkähler manifolds, Algebraic groups, Universitätsverlag Göttingen, Göttingen, 2007, pp. 1-17.

[Biq96] Olivier Biquard, Sur les équations de Nahm et la structure de Poisson des algèbres de Lie semi-simples complexes, Math. Ann. 304 (1996), no. 2, 253276.

[Bry90] Jean-Luc Brylinski, Noncommutative Ruelle-Sullivan type currents, The Grothendieck Festschrift, Vol. I, Progr. Math., vol. 86, Birkhäuser Boston, Boston, MA, 1990, pp. 477-498. 
[BSS76] Raoul Bott, Herbert Shulman, and James Stasheff, On the de Rham theory of certain classifying spaces, Advances in Math. 20 (1976), no. 1, 43-56.

[Cal79] Eugenio Calabi, Métriques kählériennes et fibrés holomorphes, Ann. Sci. École Norm. Sup. (4) 12 (1979), no. 2, 269-294.

[Cal10] Martin Callies, Dimensional reduction for the generalized Seiberg-Witten equations and the Chern-Simons-Dirac functional, Diplom in mathematics, Georg-August Universität Göttingen, 2010.

[Car51] Henri Cartan, La transgression dans un groupe de Lie et dans un espace fibré principal, Colloque de topologie (espaces fibrés), Bruxelles, 1950, Georges Thone, Liège; Masson et Cie., Paris, 1951, pp. 57-71.

[CE48] Claude Chevalley and Samuel Eilenberg, Cohomology theory of Lie groups and Lie algebras, Trans. Amer. Math. Soc. 63 (1948), 85-124.

[CFRZ15] Martin Callies, Yaël Frégier, Christopher L. Rogers, and Marco Zambon, Homotopy moment maps, arxiv preprint (2015), available as arXiv:1304.2051v2.

[Don83] Simons K. Donaldson, An application of gauge theory to four-dimensional topology, J. Differential Geom. 18 (1983), no. 2, 279-315.

[Don84] Simon K. Donaldson, Nahm's equations and the classification of monopoles, Comm. Math. Phys. 96 (1984), no. 3, 387-407.

[Don87] Irrationality and the h-cobordism conjecture, J. Differential Geom. 26 (1987), no. 1, 141-168.

[Don02] _ Floer homology groups in Yang-Mills theory, Cambridge Tracts in Mathematics, vol. 147, Cambridge University Press, Cambridge, 2002, With the assistance of M. Furuta and D. Kotschick.

[DS11] Simon K. Donaldson and Ed Segal, Gauge theory in higher dimensions, II, Surveys in differential geometry. Volume XVI. Geometry of special holonomy and related topics, Surv. Differ. Geom., vol. 16, Int. Press, Somerville, MA, 2011, pp. 1-41.

[Dup76] Johan L. Dupont, Simplicial de Rham cohomology and characteristic classes of flat bundles, Topology 15 (1976), no. 3, 233-245.

[EH78] Tohru Eguchi and Andrew J. Hanson, Asymptotically flat self-dual solutions to euclidean gravity, Phys. Lett. B 74 (1978), 249-251.

[EL95] James Eells and Luc Lemaire, Two reports on harmonic maps, World Scientific Publishing Co., Inc., River Edge, NJ, 1995. 
[Fei99] Birte Feix, Hyperkähler Metrics on Cotangent Bundles, $\mathrm{PhD}$ in mathematics, University of Cambridge, 1999.

[FL98] Paul M. N. Feehan and Thomas G. Leness, PU(2) monopoles. I. Regularity, Uhlenbeck compactness, and transversality, J. Differential Geom. 49 (1998), no. 2, 265-410.

[FL01] _ $P U(2)$ monopoles and links of top-level Seiberg-Witten moduli spaces, J. Reine Angew. Math. 538 (2001), 57-133.

[FLGZ14] Yaël Frégier, Genoux Laurent-Genoux, and Marco Zambon, A cohomological framework for homotopy moment maps, arxiv preprint (2014), available as arXiv:1409.3142.

[Flo88] Andreas Floer, An instanton-invariant for 3-manifolds, Comm. Math. Phys. 118 (1988), no. 2, 215-240.

[FRZ13] Yaël Frégier, Christopher L. Rogers, and Marco Zambon, Homotopy moment maps, arxiv preprint (2013), available as arXiv:1304.2051v1.

[Fue34] Rudolf Fueter, Die Funktionentheorie der Differentialgleichungen $\Delta u=0$ und $\Delta \Delta u=0$ mit vier reellen Variablen, Comment. Math. Helv. 7 (1934), no. 1, $307-330$.

[GHV76] Werner Greub, Stephen Halperin, and Ray Vanstone, Connections, curvature, and cohomology. Vol. III: Cohomology of Principal Bundles and Homogeneous Spaces, Academic Press, New York-London, 1976, Pure and Applied Mathematics, Vol. 47-III.

[GL88] Krzysztof Galicki and H. Blaine Lawson, Jr., Quaternionic reduction and quaternionic orbifolds, Math. Ann. 282 (1988), no. 1, 1-21.

[GS99] Victor W. Guillemin and Shlomo Sternberg, Supersymmetry and equivariant de Rham theory, Mathematics Past and Present, Springer-Verlag, Berlin, 1999.

[Hay06] Andriy Haydys, Generalized Seiberg-Witten equations and hyperKähler geometry., PhD in mathematics, Georg-August-Universität Göttingen, 2006.

[Hay08] HyperKähler and quaternionic Kähler manifolds with $S^{1}$-symmetries, J. Geom. Phys. 58 (2008), no. 3, 293-306.

[Hay12]_ Gauge theory, calibrated geometry and harmonic spinors, J. Lond. Math. Soc. (2) 86 (2012), no. 2, 482-498. 
[Hay15a] Dirac Operators in Gauge Theory, New Ideas in Low Dimensional Topology (L.H. Kauffman and V.O. Manturov, eds.), Series on Knots and Everything, World Scientific Publishing Company Pte Limited, 2015, pp. 161188.

[Hay15b]__ Fukaya-Seidel category and gauge theory, J. Symplectic Geom. 13 (2015), no. 1, 151-207.

[HH06] Katharina Habermann and Lutz Habermann, Introduction to symplectic Dirac operators, Lecture Notes in Mathematics, vol. 1887, Springer-Verlag, Berlin, 2006.

[Hit83] Nigel J. Hitchin, On the construction of monopoles, Comm. Math. Phys. 89 (1983), no. 2, 145-190.

[Hit87]_ The self-duality equations on a Riemann surface, Proc. London Math. Soc. (3) 55 (1987), no. 1, 59-126.

[HKLR87] Nigel. J. Hitchin, Anders Karlhede, Ulf Lindström, and Martin Roček, HyperKähler metrics and supersymmetry, Comm. Math. Phys. 108 (1987), no. 4, $535-589$.

[HNS09a] Sonja Hohloch, Gregor Noetzel, and Dietmar A. Salamon, Floer homology groups in hyperkähler geometry, New perspectives and challenges in symplectic field theory, CRM Proc. Lecture Notes, vol. 49, Amer. Math. Soc., Providence, RI, 2009, pp. 251-261.

[HNS09b] _ Hypercontact structures and Floer homology, Geom. Topol. 13 (2009), no. 5, 2543-2617.

[HW15] Andriy Haydys and Thomas Walpuski, A compactness theorem for the SeibergWitten equation with multiple spinors in dimension three, Geom. Funct. Anal. 25 (2015), no. 6, 1799-1821.

[KM07] Peter Kronheimer and Tomasz Mrowka, Monopoles and three-manifolds, New Mathematical Monographs, vol. 10, Cambridge University Press, Cambridge, 2007.

[KMS93] Ivan Kolářr, Peter W. Michor, and Jan Slovák, Natural operations in differential geometry, Springer-Verlag, Berlin, 1993.

[Kov96] Alexei G. Kovalev, Nahm's equations and complex adjoint orbits, Quart. J. Math. Oxford Ser. (2) 47 (1996), no. 185, 41-58. 
[Kro88] Peter B. Kronheimer, A hyperkähler structure on the cotangent bundle of a complex Lie group, preprint (1988), available as

http://www.math.harvard.edu/ kronheim/hkgc.pdf.

[Kro89]_ The construction of ALE spaces as hyper-Kähler quotients, J. Differential Geom. 29 (1989), no. 3, 665-683.

[Kro90a] _ A hyper-Kählerian structure on coadjoint orbits of a semisimple complex group, J. London Math. Soc. (2) 42 (1990), no. 2, 193-208.

[Kro90b] Instantons and the geometry of the nilpotent variety, J. Differential Geom. 32 (1990), no. 2, 473-490.

[LM89] H. Blaine Lawson, Jr. and Marie-Louise Michelsohn, Spin geometry, Princeton Mathematical Series, vol. 38, Princeton University Press, Princeton, NJ, 1989.

[Man16] Ciprian Manolescu, Pin(2)-Equivariant Seiberg-Witten Floer homology and the Triangulation Conjecture, J. Amer. Math. Soc. 29 (2016), no. 1, 147-176.

[McC01] John McCleary, A user's guide to spectral sequences, second ed., Cambridge Studies in Advanced Mathematics, vol. 58, Cambridge University Press, Cambridge, 2001.

[Mei05] Eckhard Meinrenken, Witten's formulas for intersection pairings on moduli spaces of flat G-bundles, Adv. Math. 197 (2005), no. 1, 140-197.

[MM65] John W. Milnor and John C. Moore, On the structure of Hopf algebras, Ann. of Math. (2) 81 (1965), 211-264.

[MS12] Thomas Bruun Madsen and Andrew Swann, Multi-moment maps, Adv. Math. 229 (2012), no. 4, 2287-2309.

[MS13] _ Closed forms and multi-moment maps, Geom. Dedicata 165 (2013), $25-52$.

[Nah82] Werner Nahm, The construction of all self-dual multimonopoles by the ADHM method, Monopoles in quantum field theory (Trieste, 1981), World Sci. Publishing, Singapore, 1982, pp. 87-94.

[Nak94] Hiraku Nakajima, Instantons on ALE spaces, quiver varieties, and Kac-Moody algebras, Duke Math. J. 76 (1994), no. 2, 365-416.

[Nak13] Nobuhiro Nakamura, Pin $^{-}(2)$-monopole equations and intersection forms with local coefficients of four-manifolds, Math. Ann. 357 (2013), no. 3, 915-939. 
[Pid04] Victor Ya. Pidstrygach, Hyper-Kähler manifolds and the Seiberg-Witten equations, Tr. Mat. Inst. Steklova 246 (2004), no. Algebr. Geom. Metody, Svyazi i Prilozh., 263-276.

[PT95] Victor Ya. Pidstrygach and Andrei Tyurin, Localization of Donaldson invariants along the Seiberg-Witten classes, arxiv preprint (1995), arXiv:dgga/9507004v1.

[Rog12] Christopher L. Rogers, $L_{\infty}$-algebras from multisymplectic geometry, Lett. Math. Phys. 100 (2012), no. 1, 29-50.

[Sal89] Simon Salamon, Riemannian geometry and holonomy groups, Pitman Research Notes in Mathematics Series, vol. 201, Longman Scientific \& Technical, Harlow, 1989 .

[Sal13] Dietmar Salamon, The three-dimensional Fueter equation and divergence-free frames, Abh. Math. Semin. Univ. Hambg. 83 (2013), no. 1, 1-28.

[Sch10] Henrik Schumacher, Generalized Seiberg-Witten equations: Swann bundles and $L^{\infty}$-estimates, Diplom in mathematics, Georg-August-Universität Göttingen, 2010 .

[Seg74] Graeme Segal, Categories and cohomology theories, Topology 13 (1974), 293-312.

[SW94] Nathan Seiberg and Edward Witten, Electric-magnetic duality, monopole condensation, and confinement in $N=2$ supersymmetric Yang-Mills theory, Nuclear Phys. B 426 (1994), no. 1, 19-52.

[Swa91] Andrew Swann, Hyper-Kähler and quaternionic Kähler geometry, Math. Ann. 289 (1991), no. 3, 421-450.

[SZ15] Carlos Shahbazi and Marco Zambon, Products of multisymplectic manifolds and homotopy moment maps, arxiv preprint (2015), available as arXiv:1504.08194.

[Tau99] Clifford H. Taubes, Nonlinear generalizations of a 3-manifold's Dirac operator, Trends in mathematical physics (Knoxville, TN, 1998), AMS/IP Stud. Adv. Math., vol. 13, Amer. Math. Soc., Providence, RI, 1999, pp. 475-486.

[Tau13a] Compactness theorems for $S L(2 ; \mathbb{C})$ generalizations of the 4dimensional anti-self dual equations, arxiv preprint (2013).

[Tau13b] $\quad P S L(2 ; \mathbb{C})$ connections on 3-manifolds with $L^{2}$ bounds on curvature, Camb. J. Math. 1 (2013), no. 2, 239-397. 
[Tau14] _ The zero loci of z/2 harmonic spinors in dimension 2, 3 and 4, arxiv preprint (2014), available as arXiv:1407.6206.

[Tel00] Andrei Teleman, Moduli spaces of PU(2)-monopoles, Asian J. Math. 4 (2000), no. 2, 391-435.

[VW94] Cumrun Vafa and Edward Witten, A strong coupling test of S-duality, Nuclear Phys. B 431 (1994), no. 1-2, 3-77.

[Wal15] Thomas Walpuski, A compactness theorem for fueter sections, arxiv preprint (2015), available as arXiv:1507.03258v1.

[Xin96] Yuanlong Xin, Geometry of harmonic maps, Progress in Nonlinear Differential Equations and their Applications, 23, Birkhäuser Boston, Inc., Boston, MA, 1996.

[Zen12] Raphael Zentner, On higher rank instantons and the monopole cobordism program, Q. J. Math. 63 (2012), no. 1, 227-256. 